Desenvolvimento e teste de esquemas "upwind" de alta resolução e suas aplicações em escoamentos incompressíveis com superfícies livres

Rafael Alves Bonfim de Queiroz 


\title{
Desenvolvimento e teste de esquemas "upwind" de alta resolução e suas aplicações em escoamentos incompressíveis com superfícies livres ${ }^{1}$
}

\section{Rafael Alves Bonfim de Queiroz}

\author{
Orientador: Prof. Dr. Valdemir Garcia Ferreira
}

Dissertação apresentada ao Instituto de Ciências Matemáticas e de Computação - ICMC/USP como parte dos requisitos para obtenção do título de Mestre em Ciências Ciências de Computação e Matemática Computacional.

\section{USP - São Carlos \\ Março/2009}

\footnotetext{
${ }^{1}$ Trabalho realizado com o apoio da FAPESP através do processo 06/05910-1.
} 
Deus onipotente e misericordioso olhai com bondade este trabalho científico e fazei que ele atinja os fins que vos agradam. Por Cristo, nosso Senhor. 



\section{Agradecimentos}

Primeiramente a Deus, pelo dom da vida e novo alento.

Ao meu papai Arlindo e à minha mamãe Salete, pelo amor e motivação para os meus estudos. Aos meus irmãos Danilo, João Marcos e Lucas, às minhas irmãs Aline e Amanda, pelo carinho.

Ao amigo e professor Dr. Valdemir Garcia Ferreira do ICMC-USP, pela orientação e confiança.

Ao meu padrinho Hércules e às minhas madrinhas Elza e Leonice, por toda a ajuda e torcida.

À minha namorada Amanda, por me fazer ainda mais feliz.

Ao amigo e professor Dr. João Fernando Marar da UNESP - Bauru, pelos ensinamentos e orientação em iniciação científica.

Aos amigos Cássio M. Oishi, Fernando Akira Kurokawa, Fernando Pacanelli Martins e Rafael Gigena Cuenca, pelos trabalhos em equipe e toda ajuda.

À minha amiga Analice Costacurta Brandi, pelos materiais didáticos disponibilizados e disposição em explicar a sua dissertação para mim.

Ao professor Dr. Raúl Antonino Feijóo do LNCC e ao professor Dr. Philippe Remy Bernard Devloo da UNICAMP, pela participação como membros da banca da minha Defesa de Mestrado.

Ao professor Dr. Fabrício Simeoni de Sousa do ICMC-USP e ao professor Dr. Paulo Seleghim Júnior da EESC - USP, pela participação como membros da banca do meu Exame de Qualificação.

Ao professor Dr. João Luiz Filgueiras de Azevedo do CTA/IAE, pelo estímulo e interesse que despertou em mim pela área de CFD através de sua palestra proferida no XXIX CNMAC em 2006.

A todos os professores e alunos dos grupos de pesquisa do LCAD e funcionários do ICMC-USP, pela saudável convivência e apoio dado durante a realização deste trabalho.

À Fundação de Amparo à Pesquisa do Estado de São Paulo (FAPESP), pelo suporte financeiro para a realização do meu projeto de pesquisa (número do processo: 06/05910-1). 



\section{Resumo}

VEste trabalho são apresentados os resultados do desenvolvimento e teste numérica em leis de conservação gerais e problemas em dinâmica dos fluidos. Em particular, são derivados dois novos esquemas: o ALUS ("Adaptive Linear Upwind Scheme") e o TOPUS ("Third-Order Polynomial Upwind Scheme"). Esses esquemas são testados no transporte de escalares, em equações 1D tipo convecção-difusão, em sistemas hiperbólicos 1D, nas equações de Euler 2D da dinâmica dos gases e nas equações de Navier-Stokes incompressíveis 2D/3D. Os esquemas são então associados a uma modelagem algébrica não linear para a simulação de problemas de escoamentos incompressíveis turbulentos $2 \mathrm{D}$ com/sem superfícies livres.

Palavras-chave: Esquemas "upwind" de alta resolução; Modelagem da turbulência; Escoamentos compressíveis e incompressíveis; Equações de Navier-Stokes instantâneas e médias; Leis de conservação; Simulação numérica; Método de diferenças finitas; Escoamentos com superfícies livres; Esquemas TVD; Transporte convectivo. 



\section{Abstract}

$\mathrm{T}^{\mathrm{N}}$ this work, results of the development and testing of high-resolution 1 upwind schemes for controlling of the numerical diffusion for general conservation laws and fluid dynamics problems are presented. In particular, two new high-resolution upwind schemes are derived, namely, the ALUS (Adaptive Linear Upwind Scheme) and the TOPUS (Third-Order Polynomial Upwind Scheme). These schemes are tested in scalar transport, 1D convection-diffusion equations, 1D hyperbolic systems, 2D Euler equations of the gas dynamics, and in 2D/3D incompressible Navier-Stokes equations. The schemes are then combined with a nonlinear Reynolds stress algebraic equation model for the simulation of 2D incompressible turbulent flows with/without free surfaces.

Key words: High-resolution upwind schemes; Turbulence modelling; Compressible and incompressible flows; Averaged and instantaneous Navier-Stokes equations; conservation laws; Numerical simulation; Finite difference method; Free surface flow; TVD schemes; Convective transport. 



\section{Sumário}

Resumo

Abstract

1 Introdução 1

2 Modelagem Matemática - Tema da Pesquisa 5

2.1 Equação de Advecção 1D . . . . . . . . . . . . . . . . . . . . . 5

2.2 Equação de Convecção-Difusão 1D . . . . . . . . . . . . . . . . . . . 5

2.3 Equações de Águas Rasas $1 \mathrm{D} \ldots$. . . . . . . . . . . . . . . . . . . . . . . 7

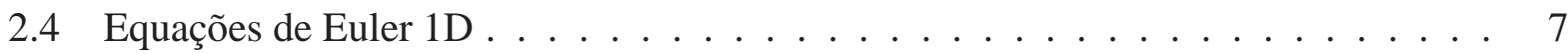

2.5 Equações de Euler 2D . . . . . . . . . . . . . . . . . . . . . . . . . . . . . . . . .

2.6 Equações Instantâneas de Navier-Stokes . . . . . . . . . . . . . . . . . . . . 8

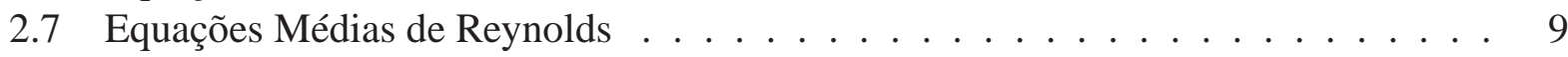

2.8 Modelagem $\kappa-\varepsilon$ da Turbulência . . . . . . . . . . . . . . . . . 10

2.9 Modelagem Não Linear para Tensões de Reynolds . . . . . . . . . . . . . . . . . . 11

2.10 Modelo Oldroyd-B . . . . . . . . . . . . . . . . . . . . 12

3 Esquemas “Upwind” de Alta Resolução 15

3.1 Formulação de Variáveis Normalizadas . . . . . . . . . . . . . . . . . . 15

3.2 Esquema TOPUS . . . . . . . . . . . . . . . . . . 17

3.3 Esquema ALUS . . . . . . . . . . . . . . . . 21

4 Modelagem Computacional $\quad 23$

4.1 Discretização dos Termos Convectivos . . . . . . . . . . . . . . . . . . . . . . 24

4.2 Algoritmo para Simulação de Escoamentos Incompressíveis Laminares . . . . . . 29

4.3 Algoritmo para Simulação de Escoamentos Incompressíveis Turbulentos . . . . . . 30

5 Resultados Numéricos 1D $\quad 33$

5.1 Advecção Linear . . . . . . . . . . . . . . . . . . . . . . . . . . . . . . 33

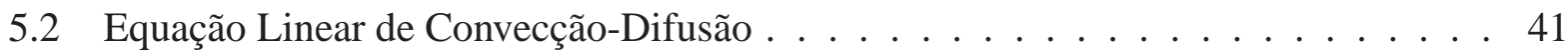

5.3 Problemas de Riemann para a Equação de Burgers . . . . . . . . . . . . . . . . 41

5.4 Equação de Buckley-Leverett . . . . . . . . . . . . . . . . . . . . . . . 48

5.5 Equação de Águas Rasas . . . . . . . . . . . . . . . . . . . . . . . . . . . . . 49 
5.6 Problemas de Riemann para as Equações de Euler . . . . . . . . . . . . . . . . . . 52

5.6 .1 Tubo de Choque de Sod . . . . . . . . . . . . . . . . . 52

5.6 .2 Tubo de Choque de Shu-Osher . . . . . . . . . . . . . . 52

5.6.3 Tubo de Choque de Shu-Osher Modificado . . . . . . . . . . . . . 55

5.6 .4 Tubos de Choque de Toro ..................... 55

6 Resultados Numéricos 2D e Aplicações 59

6.1 Problemas Incompressíveis . . . . . . . . . . . . . . . . . . . . . 59

6.1.1 Escoamento de Poiseuille em Regime Laminar . . . . . . . . . . . . . . . . . 59

6.1 .2 Escoamento numa Expansão Brusca . . . . . . . . . . . . . . . . . . . 62

6.1.3 Colapso de uma Coluna de Fluido . . . . . . . . . . . . . . . . . 70

6.1.4 Jato Livre sobre uma Superfície Rígida Impermeável . . . . . . . . . . . . 74

6.1.5 Aplicação: Escoamentos de um Fluido Viscoelástico . . . . . . . . . . . . . 80

6.1.6 Aplicação: Problema "Sluice Gate" . . . . . . . . . . . . . . . . . 86

6.1.7 Aplicação: Jato num Corpo de Fluido em Repouso . . . . . . . . . . . . . 86

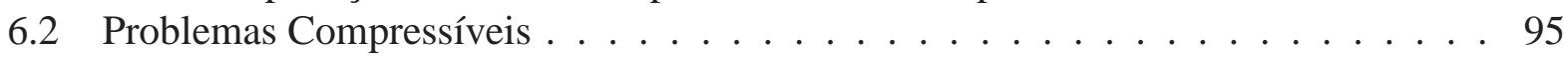

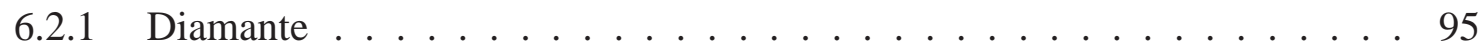

6.2 .2 RAE $2822 \ldots \ldots \ldots \ldots \ldots \ldots$

6.2 .3 NACA $0012 \ldots \ldots \ldots \ldots$

7 Resultados Numéricos 3D e Aplicações $\quad 101$

7.1 Colapso de um Bloco de Fluido . . . . . . . . . . . . . . . . . . . . 101

7.2 Ressalto Hidráulico Circular . . . . . . . . . . . . . . . . . . . . 102

7.3 Jatos Oscilantes . . . . . . . . . . . . . . . . . . . . . . . . . . . . . . . . . . . . . . . . . . . . . . . . . .

7.4 Interação Fluido-Estrutura . . . . . . . . . . . . . . . . . . . 108

8 Contribuições do Autor $\quad 109$

8.1 Artigos Publicados e Submetidos em Periódicos . . . . . . . . . . . . . . . . . 109

8.2 Artigos Publicados em Anais de Eventos . . . . . . . . . . . . . . . . 111

$\begin{array}{ll}\text { Conclusões e Trabalhos Futuros } & 113\end{array}$

A Demonstração da Propriedade TVD para o TOPUS com $\alpha=2 \quad 115$

B Limitador de Fluxo Flexível e Simétrico Baseado no Esquema TOPUS 117

C Formulação Espacial e Variável Normalizada dos Esquemas ALUS e TOPUS 119

D Limitador TOPUS para a simulação de escoamentos aerodinâmicos compressíveis 121

$\begin{array}{lr}\text { Referências Bibliográficas } & 123\end{array}$ 


\section{Lista de Figuras}

3.1 Posições $\mathrm{D}, \mathrm{R}$ e $\mathrm{U}$ em relação a face computacional $f$, o sinal da velocidade $v_{f}$ fornece o sentido do escoamento. . . . . . . . . . . . . . . . . . . 15

3.2 Metodologia NVF: (a) Esquemas convectivos clássicos no NVD, (b) Critério CBC. 16

3.3 Curvas características do esquema TOPUS: (a) região TVD de Harten [61], (b) limitador na região TVD de Sweby [139]. . . . . . . . . . . . . . . . . . . 21

3.4 Curvas características do esquema ALUS: (a) região TVD de Harten [61], (b) limitador na região TVD de Sweby [139]. . . . . . . . . . . . . . . . . . 22

4.1 Célula computacional mostrando o ponto $P$ de discretização dos termos convectivos, seus vizinhos, as faces envolvidas $f$ e $g$ na aproximação e a variável convectada $\phi$ sendo transportada com velocidade $v_{f}$ na direção $y . \ldots . \ldots . . . .24$

5.1 Simulação do caso 1 com número de Courant $\theta=0.05$ - Resultados numéricos para advecção de escalar usando os esquemas ADBQUICKEST, ALUS, FOU, Lax-Wendroff, TOPUS e VONOS . . . . . . . . . . . . . . . . . . . 35

5.2 Simulação do caso $1 \mathrm{com}$ número de Courant $\theta=0.5$ - Resultados numéricos para advecção de escalar usando os esquemas ADBQUICKEST, ALUS, FOU, Lax-Wendroff, TOPUS e VONOS. . . . . . . . . . . . . . . . . . . . 36

5.3 Simulação do caso 2 com número de Courant $\theta=0.05$ - Resultados numéricos para advecção de escalar usando os esquemas ADBQUICKEST, ALUS, FOU, Lax-Wendroff, TOPUS e VONOS. . . . . . . . . . . . . . . . . . . . . . . 37

5.4 Simulação do caso 2 com número de Courant $\theta=0.5$ - Resultados numéricos para advecção de escalar usando os esquemas ADBQUICKEST, ALUS, FOU, Lax-Wendroff, TOPUS e VONOS. . . . . . . . . . . . . . . . . . . . . . . 38

5.5 Simulação do caso 3 com número de Courant $\theta=0.05-$ Resultados numéricos para advecção de escalar usando os esquemas ADBQUICKEST, ALUS, FOU, Lax-Wendroff, TOPUS e VONOS. . . . . . . . . . . . . . . . . . . . . . 39

5.6 Simulação do caso 3 com número de Courant $\theta=0.5$ - Resultados numéricos para advecção de escalar usando os esquemas ADBQUICKEST, ALUS, FOU, Lax-Wendroff, TOPUS e VONOS. . . . . . . . . . . . . . . . . . . . . 40

5.7 Soluções exatas ("quadrados") e resultados numéricos ("estrelas") para o problema da camada de limite obtidos com a utilização de esquemas "upwind" de alta resolução. . . . . . . . . . . . . . . . . . . . . . . . . . 42

5.8 Caso 1: Comparação entre as soluções exata e numéricas da equação de Burgers. 43 
5.9 Caso 2: Soluções numéricas da equação de Burgers com viscosidade. . . . . . . . 44

5.10 Caso 3: Soluções numéricas da equação de Burgers com viscosidade. . . . . . . . 45

5.11 Simulação numérica de uma onda de choque usando o ALUS: (a) comparação entre as soluções numérica e exata; (b) perfil transiente da solução numérica. . . . 46

5.12 Simulação numérica de uma onda de choque usando o TOPUS: (a) comparação entre as soluções numérica e exata; (b) perfil transiente da solução numérica. . . . 46

5.13 Simulação numérica de uma onda de rarefação usando os esquemas convectivos ALUS, Superbee, TOPUS e VONOS. . . . . . . . . . . . . . . . . 47

5.14 Resultados numéricos da equação de Buckley-Leverett: (a) caso 1; (b) caso 2. . . . 48

5.15 Soluções numéricas e de referência para a profundidade $h$ no problema "dam-break". 50

5.16 Soluções numéricas e de referência para a vazão hu no problema "dam-break". . . 51

5.17 Soluções numéricas do tubo de choque de Sod obtidas com os esquemas ADBQUICKEST, ALUS, Lax-Wendroff, SMART, Superbee e TOPUS. . . . . . . . . . 53

5.18 Soluções numéricas do tubo de choque de Shu-Osher obtidas com os esquemas ADBQUICKEST, ALUS, Lax-Wendroff, SMART, Superbee e TOPUS. . . . . . . . 54

5.19 Soluções numéricas do tubo de choque de Shu-Osher modificado obtidas com os esquemas ADBQUICKEST, ALUS, ARORA, MINMOD, Superbee e TOPUS. . . 56

5.20 Caso 1: Soluções numéricas do tubo de choque de Toro obtidas com os esquemas ADBQUICKEST, ALUS, ARORA, MINMOD, SMART e TOPUS. . . . . . . . . 57

5.21 Caso 2: Soluções numéricas do tubo de choque de Toro obtidas com os esquemas ADBQUICKEST, ALUS, ARORA, SMART, Superbee e TOPUS. . . . . . . . . 58

6.1 Comparação entre as soluções numéricas ("estrelas") e semi-analíticas ("linhas contínuas") para o escoamento de Poiseuille. . . . . . . . . . . . . . . 60 60

6.2 Resultados numéricos para a velocidade $u$ obtidos nas simulações do escoamento de Poiseuille usando o esquema TOPUS e três malhas diferentes a Reynolds $R e=$

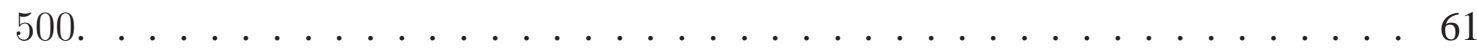

6.3 Geometria para o problema confinado numa expansão brusca. . . . . . . . . . . . . 63

6.4 Resultados numéricos para a velocidade $u$ obtidos nas simulações do escoamento numa expansão brusca a Reynolds $R e=100$. . . . . . . . . . . . . . . . . . . . 64

6.5 Resultados numéricos para a velocidade $u$ obtidos nas simulações do escoamento numa expansão brusca a Reynolds $R e=800$. . . . . . . . . . . . . . . . 65

6.6 Perfil da componente $u$ da velocidade do problema da expansão brusca quando $R e=600$, mostrando a melhoria da solução numérica com o refinamento da malha. 66

6.7 Resultados numéricos do campo de velocidade na direção $x$ obtidos nas simulações do problema da expansão brusca usando o modelo $\kappa-\varepsilon$. . . . . . . . . . . . . . . 68

6.8 Resultados numéricos do campo de velocidade na direção $x$ obtidos nas simulações do problema da expansão brusca usando o modelo algébrico RSAEM. . . . . . . . 69

6.9 Geometria do problema do colapso de uma coluna de fluido: $a=0.05 \mathrm{~m}$ e $b=0.1 \mathrm{~m} .70$

6.10 Distribuição do campo de pressão obtida na simulação do problema do colapso de coluna de fluido 2D em regime turbulento usando os esquemas ALUS e TOPUS. 71

6.11 Comparação entre os dados experimentais e os resultados numéricos obtidos nas simulações usando o esquema ALUS com e sem modelagem de turbulência. . . . . 72

6.12 Comparação entre os dados experimentais e os resultados numéricos obtidos nas simulações usando o esquema TOPUS com e sem modelagem de turbulência. . . . 73

6.13 Geometria de um jato livre incidindo sobre um contorno rígido. . . . . . . . . . . 75 
6.14 Resultados numéricos para o campo de pressão do escoamento em regime laminar de um jato livre incidindo sobre um contorno rígido. . . . . . . . . . . . . 75

6.15 Comparação entre a solução analítica de Watson e as soluções numéricas obtidas com os esquemas ADBQUICKEST, ALUS, CUBISTA e TOPUS no caso laminar,

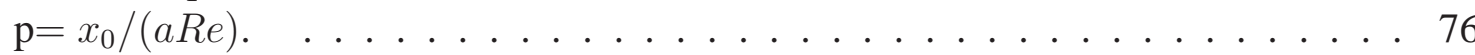

6.16 Comparação entre a solução analítica de Watson e os resultados numéricos obtidos com os esquemas ADBQUICKEST, ALUS, CUBISTA e TOPUS combinados com o modelo $\kappa-\varepsilon, \mathrm{r}=x_{0} /\left(a R e^{1 / 4}\right) \ldots \ldots \ldots \ldots \ldots \ldots$

6.17 Comparação entre a solução analítica de Watson e os resultados numéricos obtidos com os esquemas ADBQUICKEST, ALUS, CUBISTA e TOPUS combinados com o modelo RSAEM, $\mathrm{r}=x_{0} /\left(a R e^{1 / 4}\right) \ldots \ldots \ldots \ldots \ldots \ldots$

6.18 Soluções numéricas obtidas na simulação com o esquema TOPUS no instante $t=$ $2.175 s$ na malha de $200 \times 40$ células a Reynolds $R e=0.01$. . . . . . . . . . . 8

6.19 Comparação entre as soluções numéricas, obtidas com a utilização do esquema TOPUS a Reynolds $R e=0.01$ em diferentes malhas, e analíticas. . . . . . . . . . 82

6.20 Resultados numéricos da componente $v$ da velocidade obtidos usando o esquema TOPUS a Reynolds $R e=0.15$ e a Weissenberg $W e=2.5$. . . . . . . . . . . . 86

6.21 Continuação da Figura 6.20 . . . . . . . . . . . . . . . . . . . 87

6.22 Geometria do problema de um jato penetrando num corpo de fluido. . . . . . . . . 88

6.23 Evolução dos contornos do campo de pressão do problema "sluice gate". . . . . . . 89

6.24 Evolução dos contornos da componente de velocidade $u$ do problema "sluice gate". 90

6.25 Evolução dos contornos da componente de velocidade $v$ do problema "sluice gate". 91

6.26 Contornos de pressão de um jato penetrando numa porção de fluido em repouso. . . 92

6.27 Contornos da componente $u$ da velocidade de um jato penetrando numa porção de fluido em repouso. . . . . . . . . . . . . . . . . . 93

6.28 Contornos da componente $v$ da velocidade de um jato penetrando numa porção de fluido em repouso. . . . . . . . . . . . . . . . . . . . . . . . 94 94

6.29 Convergência do método numérico na simulação do escoamento supersônico ao redor de um aerofólio diamante. . . . . . . . . . . . . . . . . . 96

6.30 Simulação do escoamento transônico ao redor do aerofólio RAE 2822: (a) análise de convergência; (b) comparação entre os resultados numéricos e dado experimental para o coeficiente de pressão Cp. . . . . . . . . . . . . . . . . . 96

6.31 Resultados numéricos da distribuição de pressão e Mach ao redor do aerofólio RAE 2822. . . . . . . . . . . . . . . . . . . . . . . . . . . . 97

6.32 Resultados numéricos da distribuição da pressão P: (a) ao redor do aerofólio RAE 2822; (b) detalhe da região do choque. . . . . . . . . . . . . . . . . . . . 97

6.33 Resultados numéricos da distribuição Mach: (a) ao redor do aerofólio RAE 2822; (b) detalhe da região do choque. . . . . . . . . . . . . . . . . . . . . . . 97

6.34 Resultados numéricos da distribuição de densidade $\rho$ : (a) ao redor do aerofólio RAE 2822; (b) detalhe da região do choque. . . . . . . . . . . . . . . . . . . 98

6.35 Comparação entre os resultados numéricos e dado experimental para o coeficiente de pressão Cp ao redor do aerofólio NACA 0012 a Mach 0.3 e ângulo de ataque $0^{\circ}$.

6.36 Comparação entre os resultados numéricos e dado experimental para o coeficiente de pressão $\mathrm{Cp}$ ao redor do aerofólio NACA 0012 a Mach 0.5 e ângulo de ataque $8^{\circ}$. 
6.37 Comparação entre os resultados numéricos e dado experimental para o coeficiente de pressão Cp ao redor do aerofólio NACA 0012 a Mach 0.75 e ângulo de ataque $4^{\circ} .99$

7.1 Solução numérica do colapso de um bloco de fluido sob à ação da gravidade utilizando o esquema TOPUS. . . . . . . . . . . . . . . . . 102

7.2 Distribuição da pressão calculada na simulação do colapso de um bloco de fluido usando os esquemas ALUS e TOPUS. . . . . . . . . . . . . . . 103

7.3 Comparação entre a frente principal $x_{\max }$ do fluido, em função do tempo, calculada pelas soluções numéricas e experimentais do colapso de um bloco de fluido. . . . . 104

7.4 Resultado numérico fornecido pelo código "Freeflow-3D" equipado o esquema "upwind" de primeira ordem. . . . . . . . . . . . . . . . . . . 105

7.5 Comparação qualitativa entre os resultados numérico e experimental do ressalto hidráulico circular, $R e=150 \ldots \ldots \ldots \ldots$. . . . . . . . . . . . . . . . . . . . .

7.6 Comparação qualitativa entre os resultados numérico e experimental do ressalto hidráulico circular em regime de transição para a turbulência, $R e=1.0 \times 10^{3} \ldots$. 106

7.7 Soluções numéricas para escoamentos de jatos oscilantes. . . . . . . . . . . . . 107

7.8 Solução numérica do choque de uma onda contra um obstáculo rígido. . . . . . . . 108

B.1 Curvas características do limitador FSFL na região TVD de Sweby [139]. . . . . 118 


\section{Lista de Tabelas}

5.1 Estudo da convergência de esquemas convectivos de alta resolução, associado com um método de diferenças finitas explícito, aplicado a solução da equação da camada limite. . . . . . . . . . . . . . . . . . . . . . . 43

5.2 Estudo da convergência dos esquemas ALUS e TOPUS aplicados à equação de Burgers com viscosidade $\nu=0.05 \ldots \ldots \ldots$. . . . . . . . . . . 44

6.1 Estudo da convergência dos esquemas de alta resolução, relacionados com o método implícito de Crank-Nicolson, aplicados ao problema de Poiseuille a Reynolds

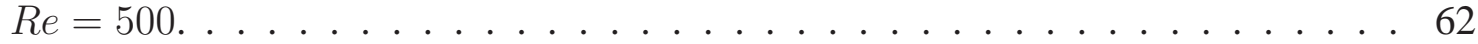

6.2 Comparação entre resultados experimentais e numéricos obtidos para o problema confinado numa expansão brusca usando diferentes esquemas convectivos e números de Reynolds. . . . . . . . . . . . . . . . . . . . . . . . . . 63

6.3 Valores da recirculação obtidos para o problema de expansão brusca no caso turbulento usando o modelo $\kappa-\varepsilon$. . . . . . . . . . . . . . . . . . . 67

6.4 Valores da recirculação obtidos para o problema de expansão brusca no caso turbulento usando o modelo RSAEM. . . . . . . . . . . . . . . . . . . . 70

6.5 Escoamento viscoelástico num canal - Erro $\|E\|_{2}$ entre as soluções numérica e analítica. . . . . . . . . . . . . . . . . . 83

6.6 Escoamento viscoelástico num canal - Taxa da convergência do método numérico na norma 2. . . . . . . . . . . . . . . . . . . . . . . . . 84

6.7 Comparação entre os resultados numéricos e teórico da inclinação da onda de choque oblíqua. . . . . . . . . . . . . . . . . . . . . 95 



\section{Lista de Símbolos e Notações}

$u, v, w$ : componentes da velocidade no sistema de coordenadas cartesianas 3D

$x, y, z$ : coordenadas espaciais no sistema de coordenadas cartesianas 3D

$\mathbf{u}_{n}$ : velocidade normal ao contorno

$\mathbf{u}_{t}$ : velocidade tangencial ao contorno

$\nu_{t}$ : viscosidade turbulenta

$\delta i$ : espaçamento da malha $(i=x, y, z)$

$\delta t$ : espaçamento temporal

$\lambda_{1}$ : tempo de relaxação

$\lambda_{2}$ : tempo de retardamento

$g_{i}: i$-ésima componente da aceleração gravitacional

$\overline{u_{i} u_{j}}$ : tensor de tensões de Reynolds

$T_{i j}$ : tensor extra tensão

$x r$ : comprimento de recirculação

$x g$ : ponto do domínio em que velocidade troca de sinal (escoamento numa expansão brusca)

Cp: coeficiente de pressão

$c$ : comprimento da corda do perfil do aerofólio

$f$ : face da célula computacional

$h$ : profundidade do fluido no canal ou altura da superfície livre ou diâmetro do injetor $p$ ou P: pressão

$t:$ tempo

D: posição "Downstream"

$L$ : escala de comprimento

$N$ : número de células computacionais

$Q$ : vazão do fluido

R: posição "Remote-Upstream"

U: posição "Upstream"

$U_{0}$ : escala de velocidade

$U_{f}$ : velocidade do fluido

$\left.F r=U_{0} / \sqrt{(} L|\mathbf{g}|\right)$ : número de Froude

$R e=\left(L U_{0}\right) / \nu$ : número de Reynolds

$W e=\left(\lambda_{1} U_{0}\right) / L$ : número de Weissenberg

$\theta=(u \delta t) / \delta x$ : número de Courant

1D: unidimensional

2D: bidimensional 
3D: tridimensional

$\alpha$ : parâmetro constante do esquema TOPUS

$\beta$ : parâmetro constante do esquema FSFL ou razão de retardamento $\left(\beta=\lambda_{2} / \lambda_{1}\right)$

$\varepsilon$ : dissipação de energia cinética

$\kappa$ : energia cinética turbulenta

$\mu$ : coeficiente de viscosidade dinâmica do fluido

$\nu$ : coeficiente de viscosidade cinemática do fluido

$\rho$ : densidade ou massa específica

$\phi$ : variável genérica não normalizada ou grandeza $(\phi=u, v, \kappa$ ou $\varepsilon)$

$\psi$ : função limitadora de fluxo

$\hat{\phi}$ : variável genérica normalizada 


\section{Lista de Acrônimos}

ADBQUICKEST - "Adaptative Bounded QUICK with Estimated Streaming Terms" ALUS -"Adaptive Linear Upwind Scheme"

CBC - "Convection Boundedness Criterion"

CDS -“Central-Differencing Scheme"

CFD -"Computational Fluid Dynamics"

CHARM - "Cubic / Parabolic High-Accuracy Resolution Method"

CLAWPACK - "Conservation LAW PACKage"

CUBISTA - "Convergent and Universally Bounded Interpolation Scheme for the Treatment of Advection"

DNS -"Direct Numerical Simulation"

EDOs - Equações Diferenciais Ordinárias

EDPs - Equações Diferencias Parciais

ENO -"Essentially Non-Oscillatory"

FOU -"First-Order Upwind"

FSFL-"Flexible and Symmetric Flux Limiter"

GENSMAC - "Generalized-Simplified-Marker-and-Cell"

HLPA -"Hybrid-Linear Parabolic Aproximation"

ISNAS -"Interpolation Scheme which is Nonoscillatory for Advected Scalars"

LES -“Large Eddy Simulation"

MINMOD - "MINimum MODulus"

MUSCL - "Monotonic Upwind Scheme for Conservation Laws"

NV -"Normalized Variable"

NVD -"Normalized Variable Diagram"

NVF -"Normalized Variable Formulation"

NVSF -"Normalized Variable and Space Formulation"

QUICK -"Quadratic Upstream Interpolation for Convective Kinematics"

QUICKEST - "QUICK with Estimated Streaming Terms"

RANS -"Reynolds-Averaged Navier-Stokes"

RSAEM - "Reynolds Stress Algebraic Equation Model"

SMART-"Sharp and Monotonic Algorithm for Realistic Transport"

SOU -"Second-Order Upwind"

TOPUS -“Third-Order Polynomial Upwind Scheme"

TVD -“Total Variation Diminishing"

VONOS -"Variable-Order Non-Oscillatory Scheme" 
WACEB -"Weighted Average Coefficients Ensuring Boundedness" WENO -"Weighted ENO" 



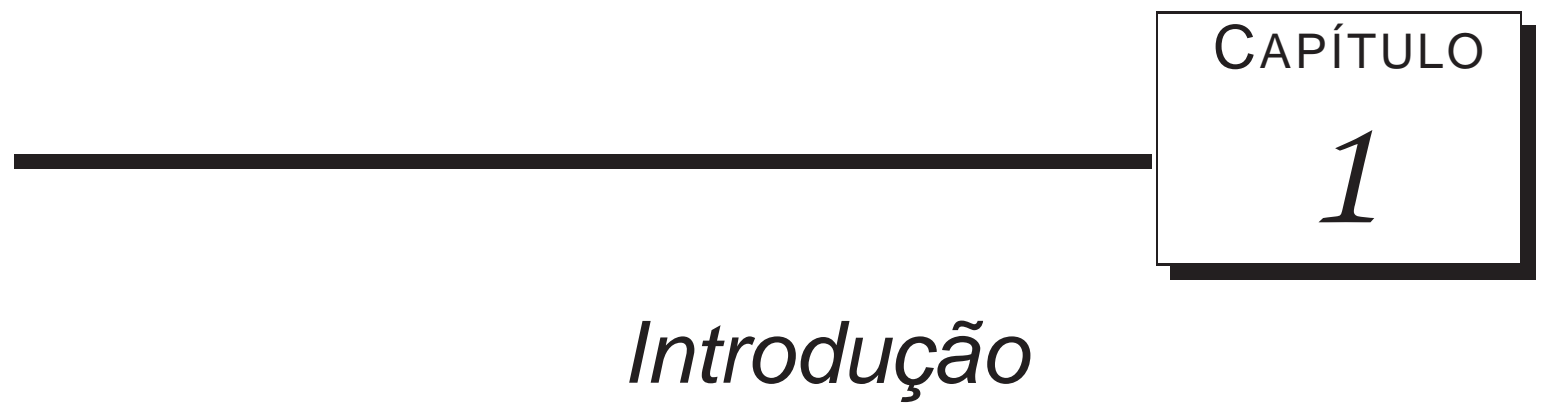

A grande maioria dos problemas de escoamento de fluidos encontrados em aplicações tecnológicas é caracterizada por possuir alto poder convectivo, especialmente aqueles no regime turbulento com superfícies livres móveis. Computar soluções numéricas representativas para essa classe de problemas, especialmente no caso dominado por convecção, é um assunto importante, desafiador e que exige o desenvolvimento de esquemas numéricos eficientes, robustos e precisos. Consequientemente, o tema tem sido uma das principais preocupações da comunidade científica moderna em CFD ("Computational Fluid Dynamics"). Fundamentalmente, dois pilares sustentam o sucesso na simulação numérica desses problemas, a saber: i) o desenvolvimento de esquemas "upwind" de alta resolução; e ii) a modelagem da turbulência.

Esquemas convectivos "upwind" de alta ordem (maior ou igual a 2) são técnicas numéricas especializadas para aproximar termos convectivos lineares (ou não lineares) de leis de conservação, tais como as equações de Euler da dinâmica dos gases ou as equações de Navier-Stokes para o movimento dos fluidos (ver, por exemplo, [157]). A idéia básica por trás destas estratégias é usar um esquema numérico tão preciso quanto possível em regiões suaves e, ao mesmo tempo, adicionar dissipação numérica controlada em regiões de gradientes elevados. Seus objetivos principais são manter estabilidade da solução numérica, capturar descontinuidades ou choques (sem a presença de oscilações numéricas ou, em casos extremos, com oscilações limitadas), ser computacionalmente simples, econômico e conseguir convergência.

Para alcançar esses objetivos, mais simplicidade na implementação, eficiência computacional e robustez, uma estratégia bastante comum (ver, por exemplo, [21, 41, 71, 85, 94, 148, 154, 161, 163]) tem sido combinar variáveis normalizadas de Leonard [81] ou limitadores de fluxo [139] com as condições para estabilidade não linear (ou critérios de limitação) TVD ("Total Variation Diminishing") de Harten [61] (ver também Sweby [139]) e CBC ("Convection Boundedness Criterion”) de Gaskell e Lau [53]. A restrição TVD visa conseguir com o tempo variação limitada de propriedades físicas e impor um auto ajuste do esquema de acordo com os gradientes locais e o critério CBC é para produzir solução limitada.

Nas últimas décadas, muitas tentativas têm sido feitas para derivar o esquema convectivo "upwind" de alta resolução perfeito, isto é, aquele que satisfaz todas as exigências mencionadas 
anteriormente, e entre elas destacam-se a proposta do esquema HLPA ("Hybrid-Linear Parabolic Approximation") de Zhu [166], do SMART ("Sharp and Monotonic Algorithm for Realistic Transport") por Gaskell e Lau [53], do WACEB ("Weighted Average Coefficient Ensuring Boundedness") por Song et al. [133], do VONOS ("Variable-Order Non-Oscillatory Scheme") de Varonos e Bergeles [152] e, mais recentemente, a proposta do CUBISTA ("Convergent and Universally Bounded Interpolation Scheme for the Treatment of Advection") de Alves et al. [3]. Por satisfazerem o critério de estabilidade $\mathrm{CBC}$, tais esquemas vêm sendo utilizados com sucesso numa variedade de aplicações (veja os trabalhos [2, 18, 57, 78, 94, 96, 98, 120, 127, 149, 163], entre muitos outros). Entretanto, a ausência do número de Courant (veja [83]) em suas formulações, além da falta de robustez em resolver problemas de evolução, e o desejo de simular problemas de escoamento de fluidos em condições adversas motivaram o desenvolvimento de uma versão limitada do esquema QUICKEST ("QUICK with Estimated Streaming Terms") de Leonard [81], chamada QUICKEST adaptativo nas referências [43, 46, 47, 49] ou ADBQUICKEST ("ADaptative Bounded QUICKEST") na referência [48].

Vale salientar que há também outra classe de esquemas de alta resolução sofisticados chamados ENO ("Essentially Non-Oscillatory") e seus relacionados WENO ("Weighted ENO”), que são apropriados para capturar descontinuidades e pontos extremos com alta ordem de precisão (ver [63]). Nesses esquemas, alta ordem é alcançada usando polinômios interpoladores de graus elevados que não garantem resultados não oscilatórios. Seus compromissos são relaxar as condições CBC/TVD e, ao invés de considerar moléculas computacionais fixas, escolher moléculas computacionais variáveis. O preço a ser pago por esse procedimento é, além da dificuldade de implementação, o número muito elevado de operações aritméticas requeridas a cada passo no tempo. A diferença entre ENO/WENO e esquemas CBC/TVD é que os esquemas ENO/WENO têm a propriedade de reter a mesma ordem de precisão espacial em todo o domínio, inclusive nas vizinhanças de descontinuidades e pontos extremos, enquanto que os esquemas CBC/TVD oferecem primeira ordem de precisão nessas regiões críticas. Em outras palavras, a dissipação numérica contida nos esquemas ENO/WENO é intrinsecamente menor que aquela nos esquemas CBC/TVD. Por outro lado, em muitas aplicações - como os escoamentos incompressíveis - segunda ordem de precisão é suficiente (ver [72]). Também, esquemas ENO/WENO são usados na simulação da turbulência com LES (“Large Eddy Simulation”) [121], em que se conclui frequientemente que eles são muitos dissipativos [43].

No que tange à modelagem de turbulência [32,159], existem poucas maneiras de se simular numericamente escoamentos incompressíveis nesse regime e, dentre elas, destacam-se três metodologias principais, a saber: a) a DNS ("Direct Numerical Simulation") [121, 134]; b) a LES [121]; e c) a RANS ("Reynolds-Averaged Navier-Stokes") [135]. Na DNS, todas as escalas da turbulência são computadas numericamente, a partir das equações instantâneas de Navier-Stokes e continuidade, sem levar em consideração o fato de que as pequenas e as grandes escalas da turbulência possuem características físicas diferentes. A necessidade de uma malha computacional excessivamente fina e de um tamanho de passo no tempo bastante reduzido exige demanda elevada de recursos computacionais, sendo a DNS limitada para problemas a baixos valores do número de Reynolds. Na LES, formulada com base num processo de filtragem das equações instantâneas de conservação, permite-se, da mesma maneira como na DNS, computar as escalas maiores ("grid-scale") da turbulência, e os efeitos das estruturas de pequenas escalas ("subgrid-scale") são modelados. Os praticantes dessa metodologia argumentam que as escalas pequenas da turbulência são mais homogêneas e isotrópicas e, portanto, fáceis de modelar. Entretanto, quando a malha 
computacional é muito fina, o número de escalas que requer modelagem torna-se muito pequeno e, conseqüentemente, a metodologia LES aproxima-se da DNS. A RANS é semelhante à LES, em que a média temporal de Reynolds, um caso particular de filtro, é adotada para simplificar a modelagem "submalha", ou seja, informações sobre flutuações turbulentas são obtidas do tensor de tensões de Reynolds.

O estudo realizado nesta pesquisa foi motivado pela necessidade de novos esquemas "upwind" de alta resolução robustos/fáceis de implementar e uma modelagem da turbulência barata. Assim, o presente trabalho de mestrado é um passo nesta direção. Em particular, ele está relacionado à solução numérica de leis de conservação gerais e escoamentos incompressíveis em regime transitório com superfícies livres móveis a números de Reynolds elevados. Atenção especial é voltada para o desenvolvimento, a análise e a implementação de esquemas de convecção e modelagem da turbulência de duas equações $[22,76]$. Outra motivação para o desenvolvimento de esquemas "upwind" está no fato de que as leis de conservação e equações da dinâmica dos fluidos resolvidas neste trabalho de mestrado aparecem em muitos problemas de interesse prático (por exemplo, aerodinâmica e hidrodinâmica).

No contexto do método das diferenças finitas (ver, por exemplo, [83]) e fazendo o uso de variáveis normalizadas e/ou limitadores de fluxo e dos critérios de limitação CBC e/ou TVD, o objetivo do presente trabalho é desenvolver, analisar e implementar esquemas convectivos "upwind" de alta resolução para aplicações na simulação numérica - direta ou por modelagem - de problemas de escoamentos de fluidos. Em particular, objetiva-se, pela aplicação dos novos esquemas TVD ALUS/TOPUS, corroborar a asserção de Arora e Roe [10] de que esquemas TVD, associados ao avanço temporal explícito de primeira ordem, têm méritos em problemas transientes.

Também se emprega neste trabalho uma combinação dos esquemas ALUS e TOPUS com a modelagem algébrica não linear de Shih et al. [123] para estimar o tensor de tensões de Reynolds mais a modelagem $\kappa-\varepsilon$ padrão de Launder e Spalding [76] para a viscosidade turbulenta. Estas metodologias foram adotadas com o objetivo de superar o alto custo associado à modelagem $\kappa-$ $\varepsilon$ para baixos Reynolds e a exigência de recursos computacionais expressivos das metodologias DNS e LES a altos Reynolds [121]. Objetiva-se ainda simular numericamente a turbulência nos escoamentos incompressíveis com superfícies livres móveis.

Os novos esquemas "upwind" e a modelagem da turbulência foram, nos contextos de diferenças finitas e variáveis primitivas, discretizados e implementados no ambiente "Freeflow" [20] para simulação de problemas de escoamentos incompressíveis, transientes e viscosos, em qualquer regime do número de Reynolds. A meta deste trabalho de mestrado foi o desenvolvimento, a análise e a implementação de esquemas TVD simples e que atendam, ao máximo, as exigências mencionadas anteriormente, cujo objetivo maior é o de simular numericamente escoamentos transientes tridimensionais (3D) com superfícies livres móveis numa faixa ampla de Reynolds.

O restante do texto está organizado em sete capítulos, como segue. No capítulo 2 são apresentados a modelagem matemática e o tema da pesquisa. O desenvolvimento dos esquemas ALUS TOPUS é discutido no capítulo 3. A modelagem computacional necessária para a compreensão do texto é resumida no capítulo 4. No capítulo 5 são aplicados (e comparados com outros esquemas bem conhecidos da literatura) os esquemas desenvolvidos em leis de conservação unidimensionais (1D). A simulação de escoamentos bidimensionais (2D) de fluidos newtonianos e viscoelásticos, incompressíveis/compressíveis, transientes e viscosos num vasto número de Reynolds é descrita no capítulo 6. No capítulo 7 são apresentados os resultados das simulações dos escoamentos transientes 3D com superfícies livres móveis. No capítulo 8 são documentados os artigos científicos 
$[30,48,50,102,105,108,109,110,112,113,114,115]$ produzidos pelo autor e seus colaboradores durante o desenvolvimento deste trabalho. Em seguida, as conclusões e propostas de trabalhos futuros são delineadas. No apêndice A é demonstrado o esquema TOPUS TVD. O desenvolvimento de um novo limitador de fluxo flexível e simétrico baseado no esquema TOPUS é descrito no apêndice B. A formulação espacial e variáveis normalizadas [33] para o ALUS e TOPUS é apresentada no apêndice C. No apêndice D é formulado o limitador TOPUS para a simulação de escoamentos aerodinâmicos compressíveis. A dissertação é encerrada com as referências bibliográficas. 


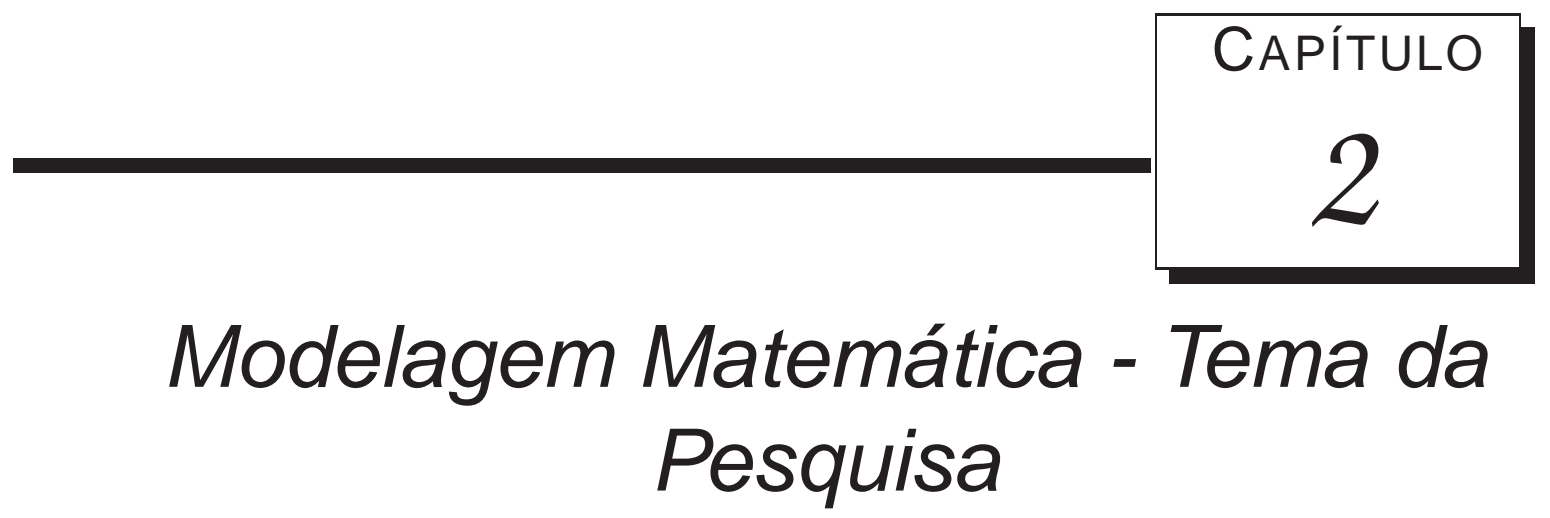

Neste capítulo são apresentadas as Equações Diferencias Parciais (EDPs) básicas para testar o desempenho dos esquemas "upwind", objeto dessa dissertação.

\subsection{Equação de Advecção 1D}

Para a simulação do transporte de escalares, o seguinte modelo é adotado:

$$
\begin{gathered}
\frac{\partial u}{\partial t}+a \frac{\partial u}{\partial x}=0 \\
u(x, 0)=u_{0}(x), \quad x \in\left[x_{L}, x_{R}\right] \\
u\left(x_{L}, t\right)=u_{L}, \quad u\left(x_{R}, t\right)=u_{R} \quad\left(u_{L} \text { e } u_{R} \text { são constantes }\right),
\end{gathered}
$$

em que $t$ é o tempo; $u$ é a variável dependente, assemelhando-se a velocidade de um escoamento num meio fluido; $a$ é constante; $u_{0}(x)$ é a condição inicial; $x_{R}$ e $x_{L}$ são, respectivamente, os extremos direito e esquerdo do domínio 1D. A solução exata de $(2.1)$ é $u(x, t)=u_{0}(x-a t)$ (ver [92]).

\subsection{Equação de Convecção-Difusão 1D}

A equação de convecção-difusão é uma EDP simples linear ou não que modela uma variedade de problemas em dinâmica dos fluidos (ver, por exemplo, $[68,158]$ ) e é propensa à formação de choques, mesmo nos casos de dados iniciais suaves. Ela serve, por exemplo, no caso da equação de Burgers, como um modelo simplificado (combinando convecção não linear e difusão linear) para o entendimento da turbulência [13]. A equação de convecção-difusão considerada neste trabalho é dada por

$$
\begin{gathered}
\frac{\partial u}{\partial t}+\frac{\partial}{\partial x}(f(u))=\nu \frac{\partial^{2} u}{\partial x^{2}}, \\
u(x, 0)=u_{0}(x), \quad x \in\left[x_{L}, x_{R}\right],
\end{gathered}
$$




$$
u\left(x_{L}, t\right)=u_{L}, \quad u\left(x_{R}, t\right)=u_{R} \quad\left(u_{L} \text { e } u_{R} \text { são constantes }\right),
$$

em que $f(u)$ é a função fluxo e $\nu$ é o coeficiente de viscosidade (constante). Assumindo em (2.4) as condições

$$
\nu=0 \quad \text { e } \quad f(u)=\frac{u^{2}}{u^{2}+0.25(1-u)^{2}},
$$

obtém-se a equação de Buckley-Leverett, uma lei geral de conservação hiperbólica 1D [1].

A equação de Burgers viscosa é obtida de (2.4) adotando

$$
\nu>0 \quad \text { e } f(u)=0.5 u^{2}
$$

e de Burgers inviscida considerando

$$
\nu=0 \quad \text { e } \quad f(u)=0.5 u^{2} .
$$

Em particular, a solução analítica em regime permanente ("steady") da equação de Burgers viscosa, para $x \in[0,1]$, com condições de contorno

$$
u(0)=\tanh \left(\frac{0.25}{\nu}\right), \quad u(1)=-\tanh \left(\frac{0.25}{\nu}\right)
$$

é dada por (ver referência [24])

$$
u(x)=\tanh \left(\frac{0.25-0.5 x}{\nu}\right) .
$$

Em específico, a solução analítica da equação de Burgers inviscida, com condição inicial

$$
u(x, 0)= \begin{cases}u_{L}, & x \in\left[x_{L}, 0\right), \\ u_{R}, & x \in\left[0, x_{R}\right],\end{cases}
$$

e de contorno

$$
u\left(x_{L}, t\right)=u_{L}, \quad u\left(x_{R}, t\right)=u_{R} \quad\left(u_{L} \text { e } u_{R} \text { são constantes }\right)
$$

tem as soluções analíticas (ver referência [1]):

- Onda de choque $\left(u_{L} \geq u_{R}\right)$

$$
u(x, t)= \begin{cases}u_{L}, & x-0.5\left(u_{L}+u_{R}\right) t \leq 0, \\ u_{R}, & x-0.5\left(u_{L}+u_{R}\right) t \geq 0 .\end{cases}
$$

- Onda de rarefação $\left(u_{L}<u_{R}\right)$

$$
u(x, t)= \begin{cases}u_{L}, & \frac{x}{t} \leq u_{L} \\ \Upsilon, & u_{L}<\frac{x}{t}<u_{R} \\ u_{R}, & \frac{x}{t} \geq u_{R}\end{cases}
$$

A equação da camada limite ("boundary layer”) é obtida considerando (2.4) com

$$
\nu>0 \quad \text { e } \quad f(u)=a u, \quad \text { a }- \text { constante. }
$$

Essa equação com $a=1$, condição inicial $u(x, 0)=0$, e condições de contornos $u(0, t)=0$ e $u(1, t)=1$ apresenta a solução analítica em regime permanente [24]

$$
u(x)=\frac{\left(1-\exp \left(\frac{x}{\nu}\right)\right)}{\left(1-\exp \left(\frac{1}{\nu}\right)\right)}, \quad x \in[0,1] .
$$




\subsection{Equações de Águas Rasas 1D}

O sistema hiperbólico de equações de águas rasas [82] é definido por

$$
\begin{gathered}
{\left[\begin{array}{c}
h \\
h u
\end{array}\right]_{t}+\left[\begin{array}{c}
u h \\
h u^{2}+\frac{1}{2} g h^{2}
\end{array}\right]_{x}=0,} \\
u(x, 0)=u_{0}(x), \quad h(x, 0)=h_{0}(x), \quad x \in\left[x_{L}, x_{R}\right],
\end{gathered}
$$

em que $h(x, t)$ é a profundidade do fluido no canal e $g$ é a constante gravitacional.

As condições de contorno adotadas, neste estudo, para resolver (2.16) são especificadas pelas condições homogêneas de Neumann.

\subsection{Equações de Euler 1D}

Estas EDPs constituem um sistema hiperbólico de leis de conservação que modela, por exemplo, o problema do tubo de choque. A equação toma a forma

$$
\begin{gathered}
\frac{\partial \mathbf{U}}{\partial t}+\frac{\partial \mathbf{F}}{\partial x}=0, \\
\mathbf{U}(x, 0)=\mathbf{U}_{0}(x), \quad x \in\left[0, x_{R}\right], \\
\mathbf{U}(0, t)=\mathbf{U}_{L}(t), \quad \mathbf{U}\left(x_{R}, t\right)=\mathbf{U}_{R}(t),
\end{gathered}
$$

em que

$$
\left\{\begin{array}{l}
\mathbf{U}=[\rho, \rho u, e]^{T} \\
\mathbf{F}=\left[\rho u, \rho u^{2}+p, u(e+p)\right]^{T} \\
e=\frac{p}{(\gamma-1)}+\frac{1}{2} \rho u^{2}
\end{array}\right.
$$

Em (2.20), $\mathbf{U}_{L}(t)$ e $\mathbf{U}_{R}(t)$ são, respectivamente, as condições de contorno em $x=0$ e $x=x_{R}$ (ver detalhes na referência [147]). Em (2.21), as variáveis $\rho, e$ e $p$ são a densidade, a energia total e a pressão, respectivamente; e a constante $\gamma=1.4$ (o gás é considerado ideal). 


\subsection{Equações de Euler 2D}

Estas EDPs modelam o escoamento de um gás compressível expressando a conservação de massa, quantidade de movimento e energia. Elas são dadas por

$$
\frac{\partial \mathbf{Q}}{\partial t}+\frac{\partial \mathbf{E}}{\partial x}+\frac{\partial \mathbf{F}}{\partial y}=0
$$

em que

$$
\left\{\begin{array}{l}
\mathbf{Q}=[\rho, \rho u, \rho v, e]^{T} \\
\mathbf{E}=\left[\rho u, \rho u^{2}+p, \rho u v,(e+p) u\right]^{T} \\
\mathbf{F}=\left[\rho v, \rho u v, \rho v^{2}+p,(e+p) v\right]^{T} \\
e=\frac{p}{(\gamma-1)}+\frac{1}{2} \rho\left(u^{2}+v^{2}\right)
\end{array}\right.
$$

Na equação (2.23), $u$ e $v$ são as componentes da velocidade. Condições de contorno periódicas, com escorregamento na superfície rígida e fronteira livre ("far-field") são adotadas para resolver (2.22). Essas condições estão bem descritas nas referências [29, 65].

\subsection{Equações Instantâneas de Navier-Stokes}

No caso em que o fluido é considerado um meio homogêneo incompressível, a massa específica $\rho(\mathbf{x}, t)=\rho_{0}$ das partículas não varia durante o seu movimento e as propriedades de transporte são constantes. As equações matemáticas das leis físicas de conservação, consideradas para a simulação de escoamentos em regime laminar, neste trabalho, são as equações instantâneas de Navier-Stokes e continuidade dadas por

$$
\begin{gathered}
\frac{\partial u_{i}}{\partial t}+\frac{\partial\left(u_{i} u_{j}\right)}{\partial x_{j}}=-\frac{\partial p}{\partial x_{i}}+\frac{1}{R e} \frac{\partial}{\partial x_{j}}\left(\frac{\partial u_{i}}{\partial x_{j}}\right)+\frac{1}{F r^{2}} g_{i}, \quad i=1,2,3, \\
\frac{\partial u_{i}}{\partial x_{i}}=0,
\end{gathered}
$$

nos quais $u_{i}$ é a $i$-ésima componente do campo de velocidade, $p$ é a pressão cinemática (pressão dividida pela massa específica), $R e=L U_{0} / \nu$ e $F r=U_{0} / \sqrt{L|\mathbf{g}|}$ são, respectivamente, os números de Reynolds e Froude, e $g_{i}$ é a $i$-ésima componente da aceleração gravitacional. O parâmetro $\nu$ é o coeficiente de viscosidade cinemática molecular (constante) do fluido dado por $\nu=\mu / \rho$ ( $\mu$ é a viscosidade dinâmica do fluido), e $U_{0}$ e $L$ são as escalas de velocidade e comprimento característicos, respectivamente. As condições de contorno [17, 42] consideradas, neste trabalho, para resolver o sistema formado por (2.24) e (2.25) são:

- Condição de contorno de entrada de fluido

$$
\mathbf{u}_{n}=U_{0}, \quad \mathbf{u}_{t}=0
$$

em que $\mathbf{u}_{n}$ é a velocidade normal ao contorno e $\mathbf{u}_{t}$ é a velocidade tangencial ao contorno; 
- Condição de contorno de saída de fluido

$$
\frac{\partial \mathbf{u}_{n}}{\partial n}=0, \quad \frac{\partial \mathbf{u}_{t}}{\partial n}=0
$$

em que $n$ e $t$ denotam direções normais e tangenciais à entrada e à saída, respectivamente;

- Condição de contorno sem escorregamento na superfície rígida

$$
\mathbf{u}_{t}=0, \quad \mathbf{u}_{n}=0
$$

- Condição de contorno com escorregamento na superfície rígida

$$
\mathbf{u}_{n}=0, \quad \frac{\partial \mathbf{u}_{t}}{\partial n}=0
$$

- Condição de contorno na superfície livre [145, 146]

$$
\begin{aligned}
\mathbf{n} \cdot(\sigma \cdot \mathbf{n}) & =0 \\
\mathbf{m} \mathbf{1} \cdot(\sigma \cdot \mathbf{n}) & =0 \\
\mathbf{m} 2 \cdot(\sigma \cdot \mathbf{n}) & =0
\end{aligned}
$$

em que $\mathbf{m} 1=\left(m 1_{x}, m 1_{y}, m 1_{z}\right)$ e $\mathbf{m} \mathbf{2}=\left(m 2_{x}, m 2_{y}, m 2_{z}\right)$ são os vetores tangentes à superfície livre, $\mathbf{n}=\left(n_{x}, n_{y}, n_{z}\right)$ é o vetor unitário normal externo à superfície e $\sigma$ é o tensor de tensões total dado por

$$
\sigma=-p \mathbf{I}+2 \mu \mathbf{D}
$$

\subsection{Equações Médias de Reynolds}

Para a simulação dos efeitos da turbulência, as leis físicas de conservação instantâneas (2.24) e (2.25), no caso 2D, são transformadas nas equações médias de Reynolds (ver referência [159] para detalhes)

$$
\begin{gathered}
\frac{\partial \bar{u}_{i}}{\partial t}+\frac{\partial\left(\bar{u}_{i} \bar{u}_{j}\right)}{\partial x_{j}}=-\frac{\partial \bar{p}}{\partial x_{i}}+\frac{1}{R e} \frac{\partial}{\partial x_{j}}\left(\frac{\partial \bar{u}_{i}}{\partial x_{j}}\right)+\frac{1}{F r^{2}} g_{i}-\frac{1}{R e} \frac{\partial\left(\overline{u_{i} u_{j}}\right)}{\partial x_{j}}, \quad i=1,2, \\
\frac{\partial \bar{u}_{i}}{\partial x_{i}}=0
\end{gathered}
$$

em que $\bar{u}_{i}$ é a i-ésima componente da velocidade média; $\bar{p}$ é a pressão média; $\overline{u_{i} u_{j}}$ é o tensor de tensões de Reynolds definido em (2.36) e (2.51), que resulta das não linearidades presentes nas equações do movimento. 


\subsection{Modelagem $\kappa-\varepsilon$ da Turbulência}

A idéia para a simulação da turbulência com a modelagem $\kappa-\varepsilon$ é acoplar às equações médias de Navier-Stokes (2.34)-(2.35) duas EDPs de transporte, para descrever a evolução da viscosidade adicional $\nu_{t}$ em (2.41). Uma dessas equações modela a distribuição de $\kappa$, a energia cinética média local do movimento flutuante, por todo o campo de escoamento; a outra governa uma característica turbulenta de dimensão diferente $\varepsilon=\nu\left(\overline{\partial u_{i} / \partial x_{j}}\right)^{2}$, a taxa média de dissipação (ou de destruição) da energia cinética. $\mathrm{O}$ conhecimento das grandezas $\kappa \mathrm{e} \varepsilon$ permite a avaliação da viscosidade turbulenta local $\nu_{t}$ em (2.41). De posse de $\kappa, \varepsilon$ e $\nu_{t}$, o tensor de tensões de Reynolds em (2.34) é estimado pela aproximação linear de Boussinesq [16] dada por

$$
\begin{aligned}
\overline{u_{i} u_{j}} & =-\nu_{t} D_{i j}+\frac{2}{3} \kappa \delta_{i j}, \quad i=1,2 \\
D_{i j} & =\frac{\partial \bar{u}_{i}}{\partial x_{j}}+\frac{\partial \bar{u}_{j}}{\partial x_{i}}
\end{aligned}
$$

Em resumo, o modelo $\kappa-\varepsilon$ é dado por (ver [159])

$$
\begin{gathered}
\frac{\partial \kappa}{\partial t}+\frac{\partial\left(\kappa u_{j}\right)}{\partial x_{j}}=\frac{1}{R e} \frac{\partial}{\partial x_{j}}\left(\left(1+\frac{\nu_{t}}{\sigma_{\kappa}}\right) \frac{\partial \kappa}{\partial x_{j}}\right)+\nu_{t} D_{i j} \frac{\partial u_{i}}{\partial x_{j}}-\varepsilon \\
\frac{\partial \varepsilon}{\partial t}+\frac{\partial\left(\varepsilon u_{j}\right)}{\partial x_{j}}=\frac{1}{R e} \frac{\partial}{\partial x_{j}}\left(\left(1+\frac{\nu_{t}}{\sigma_{\varepsilon}}\right) \frac{\partial \varepsilon}{\partial x_{j}}\right)+\frac{1}{T_{t}}\left(C_{1 \varepsilon} \nu_{t} D_{i j} \frac{\partial u_{i}}{\partial x_{j}}-C_{2 \varepsilon} \varepsilon\right), \\
T_{t}=\frac{\kappa}{\varepsilon}, \\
\nu_{t}=C_{\mu} \kappa T_{t},
\end{gathered}
$$

em que $\sigma_{\kappa}=1.0$ e $\sigma_{\varepsilon}=1.3$, que aparecem em (2.38) e (2.39), são coeficientes de difusão turbulentos; $C_{1 \varepsilon}=1.44, C_{2 \varepsilon}=1.92$ e $C_{\mu}=0.09$ são constantes empíricas. Mais detalhes desta modelagem da turbulência podem ser encontrados, por exemplo, em $[42,76]$. A seguir são descritas as condições iniciais e de contorno adotadas neste trabalho para resolver as equações médias de Reynolds com modelagem $\kappa-\varepsilon$ da turbulência.

As condições iniciais e de contorno para as variáveis médias velocidade e pressão são as mesmas descritas na seção 2.6. Neste estudo, similarmente a Brandi [17], as condições iniciais para as variáveis turbulentas são definidas por

$$
\kappa=0.08 R e, \quad \varepsilon=\frac{100 \kappa}{3} \sqrt{\frac{\kappa}{R e}} .
$$

Para as variáveis turbulentas $\kappa \mathrm{e} \varepsilon$, as condições de contorno de entrada de fluido coincidem com as condições iniciais impostas para tais variáveis em (2.42). No contorno de saída do fluido, as variáveis turbulentas são calculadas pela aplicação da condição de Neumann

$$
\frac{\partial \kappa}{\partial n}=0, \quad \frac{\partial \varepsilon}{\partial n}=0 .
$$

Próximos ao contorno rígido são aplicadas uma modificação das leis clássicas de parede (ver Durbin [35], Sondak e Pletcher [131]) para as variáveis $\kappa$ e $\varepsilon$ iguais àquelas adotadas por Brandi 
[17]. Na subcamada turbulenta, $\kappa$ e $\varepsilon$ são definidas são implementadas como (ver detalhes em Anderson et al. [5], Ferziger e Perić [51])

$$
\kappa=R e \frac{\left(u^{*}\right)^{2} \sqrt{C_{\mu}}}{C_{\mu}}, \quad \varepsilon=R e \frac{\left(u^{*}\right)^{3}}{K y},
$$

em que $u^{*}$ é a velocidade de atrito [131] e $K=0.41$ é a constante de von Kárman (ver Patel [100]). Na subcamada viscosa, a variável $\kappa$ é calculada utilizando a correção de Sondak e Pletcher [131]

$$
\kappa=R e \frac{\left(u^{*}\right)^{2} \sqrt{C_{\mu}}}{C_{\mu}}\left(\frac{y^{+}}{y_{c}^{+}}\right)^{2},
$$

em que $y^{+}$é dado por

$$
y^{+}=\frac{u^{*} y}{\nu} .
$$

Neste trabalho, $y_{c}^{+}$é calculado por $y_{c}^{+}=\frac{1}{K} \ln \left(y_{c}^{+}\right)+5$ (ver detalhes em [132]). A modelagem para a variável $\varepsilon$ na subcamada viscosa é definida por

$$
\varepsilon=\frac{\kappa}{l^{*}} \sqrt{\frac{\kappa}{R e}}
$$

em que a escala de comprimento $l^{*}$ de Norris e Reynolds [95] é calculada da seguinte forma

$$
l^{*}=\frac{K y^{2} \sqrt{R e \kappa}}{\sqrt[4]{C_{\mu}^{3}}(y \sqrt{R e \kappa}+5.3)} .
$$

As condições de contorno na superfície livre para o caso 2D turbulento são definidas por [17, 146]

$$
\begin{array}{r}
p+\frac{2}{3 R e} \kappa-\frac{2}{R e}\left(1+\nu_{t}\right)\left[\frac{\partial u}{\partial x} n_{x}^{2}+\frac{\partial v}{\partial y} n_{y}^{2}+\left(\frac{\partial u}{\partial y}+\frac{\partial v}{\partial x}\right) n_{x} n_{y}\right]=0 \\
\frac{1}{R e}\left(1+\nu_{t}\right)\left[2 \frac{\partial u}{\partial x} m_{x} n_{x}+2 \frac{\partial v}{\partial y} m_{y} n_{y}+\left(\frac{\partial u}{\partial y}+\frac{\partial v}{\partial x}\right)\left(m_{x} n_{y}+m_{y} n_{x}\right)\right]=0 .
\end{array}
$$

\subsection{Modelagem Não Linear para Tensões de Reynolds}

Nesta seção é apresentado o modelo não linear algébrico para o tensor de tensões de Reynolds proposto por Shih et al. [123]. Ele é denotado neste estudo pelo acrônimo RSAEM ("Reynolds Stress Algebraic Equation Model"). A aplicação desse modelo na simulação de escoamentos turbulentos com superfícies livres tem sido pouco explorada na literatura, e isto justifica o interesse em implementá-lo/aplicá-lo neste trabalho de pesquisa. 
Em suma, o modelo algébrico para o tensor de tensões de Reynolds é definido por

$$
\begin{aligned}
\overline{u_{i} u_{j}} & =\frac{2}{3} \kappa \delta_{i j}-\nu_{t}\left(\frac{\partial u_{i}}{\partial x_{j}}+\frac{\partial u_{j}}{\partial x_{i}}\right) \\
& +\frac{C_{\tau 1}}{A_{2}+\eta^{3}} \frac{\kappa^{3}}{\varepsilon^{2}}\left(\frac{\partial u_{i}}{\partial x_{k}} \frac{\partial u_{k}}{\partial x_{j}}+\frac{\partial u_{j}}{\partial x_{k}} \frac{\partial u_{k}}{\partial x_{i}}-\frac{2}{3} \frac{\partial u_{i}}{\partial x_{j}} \frac{\partial u_{j}}{\partial x_{i}} \delta_{i j}\right) \\
& +\frac{C_{\tau 2}}{A_{2}+\eta^{3}} \frac{\kappa^{3}}{\varepsilon^{2}}\left(\frac{\partial u_{i}}{\partial x_{k}} \frac{\partial u_{j}}{\partial x_{k}}-\frac{1}{3}\left(\frac{\partial u_{i}}{\partial x_{j}}\right)^{2} \delta_{i j}\right) \\
& +\frac{C_{\tau 3}}{A_{2}+\eta^{3}} \frac{\kappa^{3}}{\varepsilon^{2}}\left(\frac{\partial u_{k}}{\partial x_{i}} \frac{\partial u_{k}}{\partial x_{j}}-\frac{1}{3}\left(\frac{\partial u_{i}}{\partial x_{j}}\right)^{2} \delta_{i j}\right),
\end{aligned}
$$

em que

$$
\begin{aligned}
\nu_{t} & =C_{\mu}^{*} \frac{\kappa^{2}}{\varepsilon}, \quad C_{\mu}^{*}=\frac{2 / 3}{A_{1}+\eta+\alpha \xi}, \\
\xi & =\frac{\kappa \Omega}{\varepsilon}, \quad \Omega=\sqrt{2 \Omega_{i j}^{*} \Omega_{i j}^{*}}, \quad \Omega_{i j}^{*}=\frac{1}{2}\left(\frac{\partial u_{i}}{\partial x_{j}}-\frac{\partial u_{j}}{\partial x_{i}}\right), \\
\eta & =\frac{\kappa D}{\varepsilon}, \quad D=\sqrt{2 D_{i j} D_{i j}} .
\end{aligned}
$$

Na equação (2.51), $\delta_{i j}$ é o delta de Kronecker, $C_{\tau 1}=-4, C_{\tau 2}=13, C_{\tau 3}=-2$ e $A_{2}=1000$. Em (2.52), têm-se $A_{1}=1.25$ e $\alpha=0.9$ (veja os detalhes em [123]).

Para a avaliação do tensor algébrico em (2.51), utilizam-se as equações de transporte (2.38) e (2.39), respectivamente, para obter as variáveis turbulentas $\kappa$ e $\varepsilon$. As condições iniciais e de contorno adotadas para simular este modelo são as mesmas descritas na seção 2.8.

\subsection{Modelo Oldroyd-B}

Considerando a formulação EVSS ("Elastic Viscous Split Stress”) [116], o escoamento 2D de um fluido incompressível viscoelástico do tipo Oldroyd-B $[15,88]$ é modelado por

$$
\begin{gathered}
\frac{\partial u_{i}}{\partial t}+\frac{\partial\left(u_{i} u_{j}\right)}{\partial x_{j}}=-\frac{\partial p}{\partial x_{i}}+\frac{\beta}{R e} \frac{\partial}{\partial x_{j}}\left(\frac{\partial u_{i}}{\partial x_{j}}\right)+\frac{\partial T_{i j}}{\partial x_{j}}+\frac{1}{F r^{2}} g_{i}, \quad i=1,2, \\
T_{i j}+W e\left[\frac{\partial T_{i j}}{\partial t}+\frac{\partial\left(u_{k} T_{i j}\right)}{\partial x_{k}}+T_{i k} \frac{\partial u_{j}}{\partial x_{k}}+T_{j k} \frac{\partial u_{i}}{\partial x_{k}}\right]=\frac{2(1-\beta)}{R e}\left(\frac{\partial u_{i}}{\partial x_{j}}+\frac{\partial u_{j}}{\partial x_{i}}\right), \\
\frac{\partial u_{i}}{\partial x_{i}}=0,
\end{gathered}
$$

em que $T_{i j}$ é o tensor extra tensão, $W e=\lambda_{1} U_{0} / L$ é o número de Weissenberg, $\beta=\lambda_{2} / \lambda_{1}$ é a razão de retardamento, $\lambda_{1}$ é o tempo de relaxação e $\lambda_{2}$ é o tempo de retardamento.

Para resolver as EDPs (2.55), (2.56) e (2.57), neste trabalho, as condições de contorno adotadas para o campo de velocidade são iguais àquelas mencionadas na seção 2.6. No início do processo numérico, $T_{i j}=0$. Os valores das componentes do tensor $T_{i j}$ nos contornos [126] são definidos como seguem: 
- Condição de contorno de entrada de fluido

$$
T_{11}=T_{12}=T_{22}=0
$$

- Condição de contorno de saída de fluido

$$
\frac{\partial T_{11}}{\partial n}=\frac{\partial T_{12}}{\partial n}=\frac{\partial T_{22}}{\partial n}
$$

- Condições de contorno sem escorregamento na superfície rígida:

- Contorno rígido paralelo ao eixo $x$

$$
\begin{aligned}
T_{11}^{(t+\delta t)} & =\exp \left(-\frac{\delta t}{W e} t\right) T_{11}^{(t)}+\delta t\left[\frac{\partial u}{\partial y} \exp \left(-\frac{\delta t}{W e}\right) T_{12}+\frac{\partial u}{\partial y} T_{12}\right] \\
T_{12}^{(t+\delta t)} & =\exp \left(-\frac{\delta t}{W e}\right) T_{12}^{(t)}+\frac{(1-\beta)}{R e} \frac{\partial u}{\partial y}\left[1-\exp \left(-\frac{\delta t}{W e}\right)\right] \\
T_{22} & =0
\end{aligned}
$$

em que $\delta t$ é o espaçamento temporal;

- Contorno rígido paralelo ao eixo y

$$
\begin{aligned}
T_{22}^{(t+\delta t)} & =\exp \left(-\frac{\delta t}{W e}\right) T_{22}^{(t)}+\delta t\left[\frac{\partial v}{\partial x} \exp \left(-\frac{\delta t}{W e}\right) T_{12}+\frac{\partial v}{\partial x} T_{12}\right] \\
T_{12}^{(t+\delta t)} & =\exp \left(-\frac{\delta t}{W e}\right) T_{12}^{(t)}+\frac{(1-\beta)}{R e} \frac{\partial v}{\partial x}\left[1-\exp \left(-\frac{\delta t}{W e}\right)\right] \\
T_{11} & =0
\end{aligned}
$$

- Condição de contorno na superfície livre

$$
\begin{gathered}
p-\frac{2 \beta}{R e}\left[\frac{\partial u}{\partial x} n_{x}^{2}+\frac{\partial v}{\partial y} n_{y}^{2}+\left(\frac{\partial u}{\partial y}+\frac{\partial v}{\partial x}\right) n_{x} n_{y}\right]+T_{11} n_{x}^{2}+2 T_{12} n_{x} n_{y}+T_{22} n_{y}^{2}=0, \quad \text { (2.66) } \\
\frac{2 \beta}{R e}\left[\left(\frac{\partial v}{\partial y}-\frac{\partial u}{\partial x}\right) n_{x} n_{y}+\left(\frac{\partial u}{\partial y}-\frac{\partial v}{\partial x}\right)\left(n_{x}^{2}-n_{y}^{2}\right)\right]+\left(T_{22}-T_{11}\right) n_{x} n_{y}+T_{12}\left(n_{x}^{2}-n_{y}^{2}\right)=0 .
\end{gathered}
$$




\section{Estimativas de erros}

Neste trabalho, os erros entre as soluções numérica e exata são calculados pelas seguintes relações:

$$
\begin{aligned}
\|E\|_{1} & =\frac{\sum_{i=1}^{N}\left|\phi_{i, \text { exata }}-\phi_{i, \text { numérica }}\right|}{\sum_{i=1}^{N}\left|\phi_{i, \text { exata }}\right|} \\
\|E\|_{2} & =\sqrt{\frac{\sum_{i=1}^{N}\left(\phi_{i, \text { exata }}-\phi_{i, \text { numérica }}\right)^{2}}{\sum_{i=1}^{N}\left(\phi_{i, \text { exata }}\right)^{2}}} \\
\|E\|_{\infty} & =\frac{\max _{1 \leq i \leq n}\left|\phi_{i, \text { exata }}-\phi_{i, \text { numérica }}\right|}{\max _{1 \leq i \leq n}\left|\phi_{i, \text { exata }}\right|} .
\end{aligned}
$$

\section{Tema da Pesquisa}

Desenvolver, analisar e implementar esquemas "upwind" de alta precisão (maior ou igual a dois), que são CBC/TVD, para os termos convectivos das equações de transporte (2.1), (2.4), (2.16), (2.18), (2.22), (2.24), (2.34), (2.38), (2.39), (2.55) e (2.56) constituem os temas principais deste trabalho de mestrado. Além disso, outro tema relevante, aqui considerado, é analisar e implementar a aproximação de Boussinesq e uma modelagem algébrica não linear para o tensor de tensões de Reynolds visando a simulação de escoamentos incompressíveis transientes em regime turbulento com e sem superfícies livres móveis. 


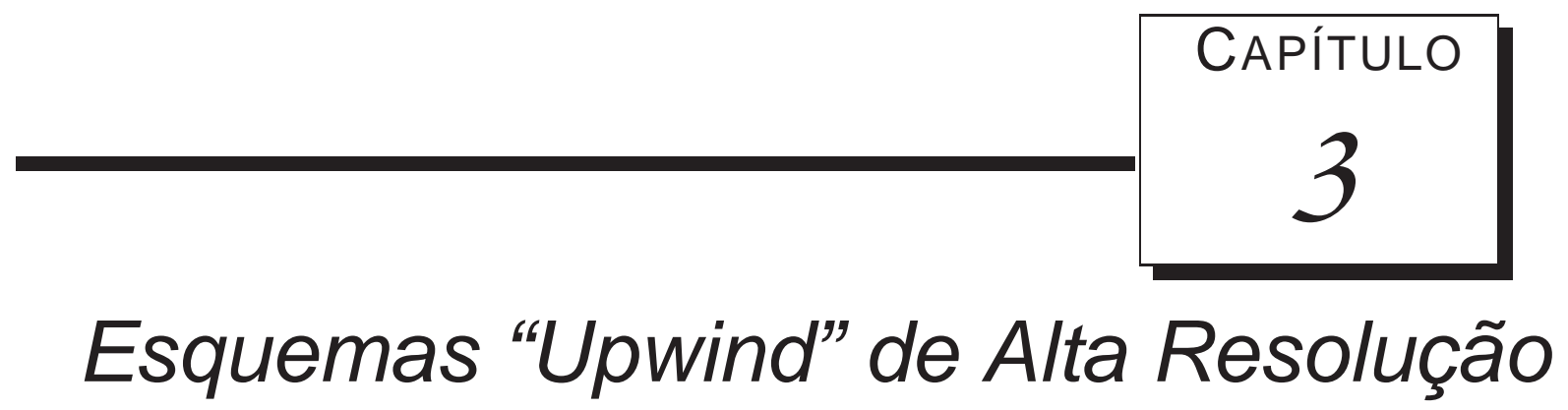

Neste capítulo são apresentados os esquemas ALUS e TOPUS desenvolvidos neste trabalho.

\subsection{Formulação de Variáveis Normalizadas}

A metodologia NVF ("Normalised Variable Formulation") foi proposta por Leonard [80] com objetivo de se obter esquemas convectivos capazes de resolver gradientes elevados e, ao mesmo tempo, manter estabilidade nas soluções numéricas. Considerando as posições D ("Downstream"), R ("Remote-Upstream") e U ("Upstream”) em relação a face computacional $f$ da molécula computacional ilustrada na Figura 3.1, uma variável genérica $\phi$ formulada em NV ("Normalised Variable") de Leonard [80] é definida como segue

$$
\hat{\phi}=\frac{\phi-\phi_{R}}{\phi_{D}-\phi_{R}}
$$

em que $\phi_{D}$ e $\phi_{R}$ são, respectivamente, os valores não normalizados da grandeza $\phi$ nos pontos D e R. Por exemplo, $\hat{\phi}_{f}=\left(\phi_{f}-\phi_{R}\right) /\left(\phi_{D}-\phi_{R}\right)$.

Leonard [80] construiu também o NVD ("Normalised Variable Diagram”) para representar a relação entre as variáveis normalizadas $\hat{\phi}_{f}$ e $\hat{\phi}_{U}$. Por exemplo, na Figura 3.2a os esquemas CDS ("Central-Differencing Scheme") [101], FOU ("First-Order Upwind") [25], QUICK ("Quadratic

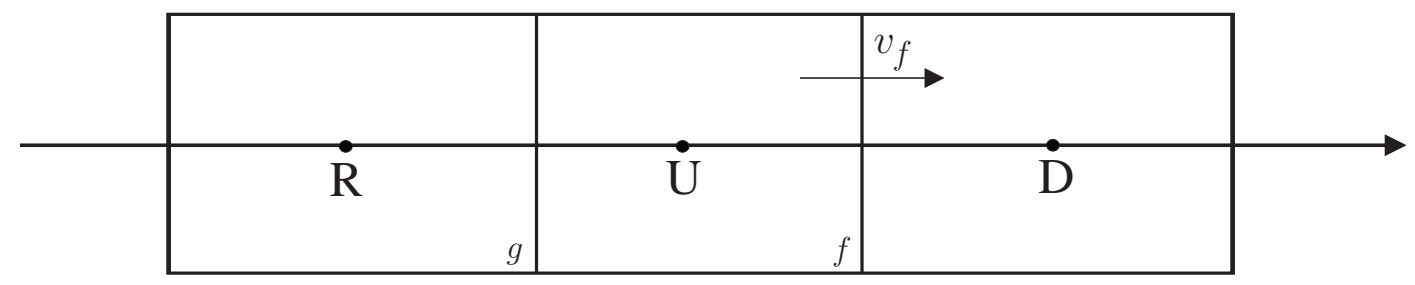

Figura 3.1: Posições D, R e U em relação a face computacional $f$, o sinal da velocidade $v_{f}$ fornece o sentido do escoamento. 
(a)

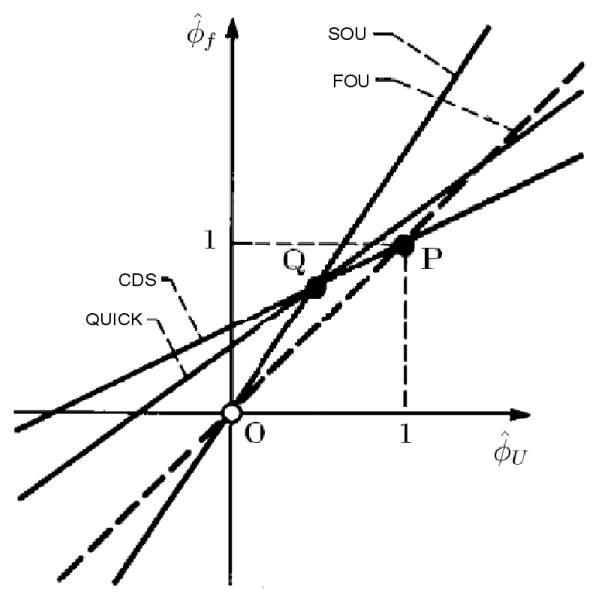

(b)

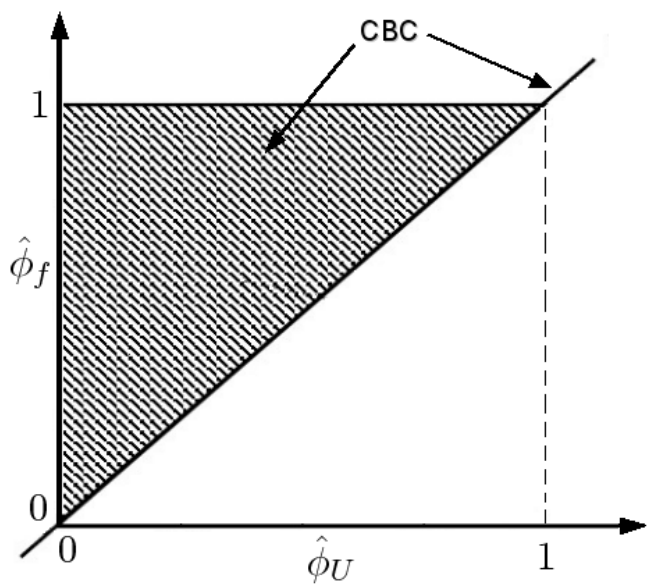

Figura 3.2: Metodologia NVF: (a) Esquemas convectivos clássicos no NVD, (b) Critério CBC.

Upstream Interpolation for Convective Kinematics") [79], SOU ("Second-Order Upwind") [125] estão representados nesse diagrama.

Segundo Leonard [80] qualquer esquema, em geral não linear, formulado em NV que passe pelos pontos $O(0,0), P(1,1)$ e $Q(0.5,0.75)$ do NVD (ver Figura 3.2a) é de segunda ordem de exatidão; e se ele também passar pelo ponto $Q$ com inclinação de 0.75 é de terceira ordem. Leonard recomenda que para valores de $\hat{\phi}_{U}$ menores que 0 ou maiores que 1 , o esquema FOU deve ser usado.

Considerando-se a importância de soluções limitadas no transporte de propriedades físicas, Gaskell e Lau [53] propuseram o critério de limitação CBC. No contexto NV, um esquema convectivo produz solução limitada se ele está inteiramente contido na região hachurada na região CBC mostrada na Figura 3.2b, isto é, o esquema deve satisfazer às seguintes condições:

$$
\begin{cases}\hat{\phi}_{f} \in\left[\hat{\phi}_{U}, 1\right], & \text { para } \hat{\phi}_{f} \in[0,1], \\ \hat{\phi}_{f}=0, & \text { para } \hat{\phi}_{U}=0, \\ \hat{\phi}_{f}=1, & \text { para } \hat{\phi}_{U}=1, \\ \hat{\phi}_{f}=\hat{\phi}_{U}, & \text { para } \hat{\phi}_{f} \notin[0,1] .\end{cases}
$$

Apesar do critério CBC de Gaskell e Lau [53] tratar o problema de estabilidade adequadamente, ele não garante convergência da solução numérica. Para convergência, as restrições TVD de Harten [61] devem ser satisfeitas. No contexto NV essas restrições são expressas por:

$$
\begin{cases}\hat{\phi}_{f} \in\left[\hat{\phi}_{U}, 2 \hat{\phi}_{U}\right] \quad \text { e } \hat{\phi}_{f} \leq 1, & \text { para } \hat{\phi}_{U} \in[0,1], \\ \hat{\phi}_{f}=\hat{\phi}_{U}, & \text { para } \hat{\phi}_{U} \notin[0,1] .\end{cases}
$$

Abaixo, estão listados alguns exemplos de esquemas "upwind" de alta resolução em váriaveis normalizadas de Leonard [80]. 
- ADBQUICKEST [49, 67]

$$
\hat{\phi}_{f}= \begin{cases}(2-\theta) \hat{\phi}_{U}, & \hat{\phi}_{U} \in[0, a), \\ \hat{\phi}_{U}+\frac{1}{2}(1-|\theta|)\left(1-\hat{\phi}_{U}\right)-\frac{1}{6}\left(1-\theta^{2}\right)\left(1-2 \hat{\phi}_{U}\right), & \hat{\phi}_{U} \in[a, b], \\ 1-\theta+\theta \hat{\phi}_{U}, & \hat{\phi}_{U} \in(b, 1], \\ \hat{\phi}_{U}, & \hat{\phi}_{U} \notin[0,1],\end{cases}
$$

em que $\theta=\frac{u \delta t}{\delta x}$ é o número de Courant; e os parâmetros $a$ e $b$ são:

$$
a=\frac{2-3|\theta|+\theta^{2}}{7-6 \theta-3|\theta|+2 \theta^{2}}, \quad b=\frac{-4+6 \theta-3|\theta|+\theta^{2}}{-5+6 \theta-3|\theta|+2 \theta^{2}} .
$$

- CUBISTA [3]

$$
\hat{\phi}_{f}= \begin{cases}1.75 \hat{\phi}_{U}, & \hat{\phi}_{U} \in[0,0.375) \\ 0.75 \hat{\phi}_{U}+0.375, & \hat{\phi}_{U} \in[0.375,0.75] \\ 0.25 \hat{\phi}_{U}+0.75, & \hat{\phi}_{U} \in(0.75,1] \\ \hat{\phi}_{U}, & \hat{\phi}_{U} \notin[0,1]\end{cases}
$$

- VONOS [152]

$$
\hat{\phi}_{f}= \begin{cases}10 \hat{\phi}_{U}, & \hat{\phi}_{U} \in\left[0, \frac{3}{74}\right), \\ 0.375\left(1+2 \hat{\phi}_{U}\right), & \hat{\phi}_{U} \in\left[\frac{3}{74}, 0.5\right), \\ 1.5 \hat{\phi}_{U}, & \hat{\phi}_{U} \in\left[0.5, \frac{2}{3}\right), \\ 1, & \hat{\phi}_{U} \in\left[\frac{2}{3}, 1\right], \\ \hat{\phi}_{U}, & \hat{\phi}_{U} \notin[0,1],\end{cases}
$$

- WACEB [133]

$$
\hat{\phi}_{f}= \begin{cases}2 \hat{\phi}_{U}, & \hat{\phi}_{U} \in[0,0.3) \\ 0.75 \hat{\phi}_{U}+0.375, & \hat{\phi}_{U} \in\left[0.3, \frac{5}{6}\right] \\ 1, & \hat{\phi}_{U} \in\left(\frac{5}{6}, 1\right] \\ \hat{\phi}_{U}, & \hat{\phi}_{U} \notin[0,1]\end{cases}
$$

\subsection{Esquema TOPUS}

Fundamentalmente, o esquema TOPUS ${ }^{1}$ pode ser visto como uma generalização do esquema SMARTER de Waterson e Deconinck [154], e sua idéia básica é usar uma combinação de esquemas de baixa e alta ordem por meio de uma função troca (o limitador). Em particular, considera-se parte de um polinômio de quarto grau inteiramente contido na região CBC e impõe-se as condições de Leonard [80], isto é, o esquema deve passar pelos pontos $O(0,0), Q(0.5,0.75)$ (para atingir segunda ordem), $P(1,1)$ e com inclinação 0.75 em $Q$ (para alcançar terceira ordem) (ver Figura 3.2a).

\footnotetext{
${ }^{1} \mathrm{Na}$ Filosofia, TOPUS é a ilha da Utopia, que segundo o pensador inglês Thomas Morus é um lugar ideal da sociedade igualitária.
} 
Vale salientar que no passado essa mesma idéia foi utilizada para a construção dos esquemas NOTABLE de Pascau e Perez [99], CHARM ("Cubic / Parabolic High-Accuracy Resolution Method") de Zhou [164, 165] e ISNAS ("Interpolation Scheme which is Nonoscillatory for Advected Scalars") de Zijlema [167, 168], e atualmente tais esquemas tem sido nomeados por Waterson e Deconinck [154] como SMARTER. Entretanto, a novidade no esquema TOPUS é a presença em sua formulação de um parâmetro livre permitindo uma generalização do esquema SMARTER.

A motivação para a construção do esquema TOPUS surgiu das deficiências do SMARTER, a saber: (i) não estar inteiramente contido na região TVD; (ii) violar o princípio de monotonicidade de Sweby [139]; (iii) não possuir em sua formulação um parâmetro livre; e (iv) fornecer resultados não satisfatórios para problemas de escoamentos incompressíveis 3D com superfícies livres móveis. Outra motivação para o desenvolvimento do TOPUS (e também do ALUS) está no fato de que as leis de conservação e equações da dinâmica dos fluidos consideradas neste trabalho aparecem com frequiência em problemas de interesse prático.

Em resumo, no contexto NV, o esquema TOPUS para o cálculo dos fluxos numéricos nas interfaces das células computacionais é definido por

$$
\hat{\phi}_{f}=a_{4} \hat{\phi}_{U}^{4}+a_{3} \hat{\phi}_{U}^{3}+a_{2} \hat{\phi}_{U}^{2}+a_{1} \hat{\phi}_{U}, \quad \hat{\phi}_{U} \in[0,1],
$$

em que $a_{1}, a_{2}, a_{3}$, e $a_{4}$ são coeficientes constantes. Nota-se que $\hat{\phi}_{f}$ passa pelo ponto $O(0,0)$ do NVD, ou seja, $\hat{\phi}_{U}=0 \Rightarrow \hat{\phi}_{f}=0$. A determinação dos coeficientes $a_{1}, a_{2}, a_{3}$ e $a_{4}$, sendo um deles livre, é feita impondo-se as outras condições de Leonard [80] para se obter terceira ordem de exatidão, isto é,

- No ponto $Q(0.5,0.75)$ :

$$
a_{4}(0.5)^{4}+a_{3}(0.5)^{3}+a_{2}(0.5)^{2}+a_{1}(0.5)=0.75,
$$

$\mathrm{ou}$

$$
a_{4}+2 a_{3}+4 a_{2}+8 a_{1}=12 .
$$

- No ponto $P(1,1)$ :

$$
a_{4}+a_{3}+a_{2}+a_{1}=1 .
$$

- No ponto $Q(0.5,0.75)$ com inclinação 0.75 :

$$
\hat{\phi}_{U}=0.5 \Rightarrow \hat{\phi}_{f}^{\prime}=4 a_{4} \hat{\phi}_{U}^{3}+3 a_{3} \hat{\phi}_{U}^{2}+2 a_{2} \hat{\phi}_{U}+a_{1}=0.75,
$$

$\mathrm{ou}$

$$
4 a_{4}(0.5)^{3}+3 a_{3}(0.5)^{2}+2 a_{2}(0.5)+a_{1}=0.75,
$$

ou

$$
2 a_{4}+3 a_{3}+4 a_{2}+4 a_{1}=3 .
$$

Considerando-se $a_{4}=\alpha$ e o sistema formado por (3.5), (3.6) e (3.7), obtêm-se os coeficientes:

$$
a_{1}=\frac{-\alpha+10}{4}, \quad a_{2}=\frac{5 \alpha-10}{4}, \quad a_{3}=-2 \alpha+1
$$


E fora da região NVD, o TOPUS coincide com o esquema FOU. Em suma, o esquema convectivo TOPUS em NV é definido por

$$
\hat{\phi}_{f}=\left\{\begin{array}{l}
\alpha \hat{\phi}_{U}^{4}+(-2 \alpha+1) \hat{\phi}_{U}^{3}+\left(\frac{5 \alpha-10}{4}\right) \hat{\phi}_{U}^{2}+\left(\frac{-\alpha+10}{4}\right) \hat{\phi}_{U}, \quad \hat{\phi}_{U} \in[0,1], \\
\hat{\phi}_{U}, \quad \hat{\phi}_{U} \notin[0,1] .
\end{array}\right.
$$

Aplicando a definição de NV (ver equação (3.1)) em (3.9), o esquema TOPUS resultante em variáveis não normalizadas é dado por

$$
\phi_{f}= \begin{cases}\phi_{R}+\left(\phi_{D}-\phi_{R}\right)\left(\alpha \hat{\phi}_{U}^{4}+(-2 \alpha+1) \hat{\phi}_{U}^{3}+\left(\frac{5 \alpha-10}{4}\right) \hat{\phi}_{U}^{2}+\left(\frac{-\alpha+10}{4}\right) \hat{\phi}_{U}\right), & \hat{\phi}_{U} \in[0,1], \\ \phi_{U}, & \hat{\phi}_{U} \notin[0,1],\end{cases}
$$

em que

$$
\hat{\phi}_{U}=\frac{\phi_{U}-\phi_{R}}{\phi_{D}-\phi_{R}} .
$$

Em (3.9), para $\alpha \in[-2,2]$, o esquema TOPUS está inteiramente contido na região CBC. Vale mencionar que, neste trabalho, apenas o TOPUS $\operatorname{com} \alpha=2$ foi testado em leis de conservação 1D e aplicado na simulação de escoamentos de fluidos 2D/3D. No caso em que $\alpha=2$, o esquema TOPUS é TVD (ver Figura 3.3a e demonstração no apêndice A). O esquema SMARTER de Waterson e Deconinck [154] é obtido fazendo-se $\alpha=0$.

O limitador de fluxo $\psi\left(r_{f}\right)$ para o esquema TOPUS é obtido reescrevendo (3.9) por (veja [139])

$$
\hat{\phi}_{f}=\hat{\phi}_{U}+\frac{1}{2} \psi\left(r_{f}\right)\left(1-\hat{\phi}_{U}\right)
$$

em que $r_{f}$ é definido por

$$
r_{f}=\frac{\hat{\phi}_{U}}{1-\hat{\phi}_{U}}
$$

Das equações (3.9) e (3.11) resulta $\psi\left(r_{f}\right)=0$, para $\hat{\phi}_{f}=\hat{\phi}_{U}$ e $\hat{\phi}_{U} \notin[0,1]$. Da equação (3.12), obtém-se $r_{f}<0$, para $\hat{\phi}_{U} \notin[0,1]$. Segue então que $\psi\left(r_{f}\right)=0$, para $r_{f}<0$. Para $r_{f} \geq 0$, considera-se $\psi\left(r_{f}\right)$ expresso como

$$
\psi\left(r_{f}\right)=2\left(a \hat{\phi}_{U}^{3}+b \hat{\phi}_{U}^{2}+c \hat{\phi}_{U}\right) .
$$

De forma que substituindo (3.13) em (3.11) obtém-se

$$
\hat{\phi}_{f}=(-a) \hat{\phi}_{U}^{4}+(a-b) \hat{\phi}_{U}^{3}+(b-c) \hat{\phi}_{U}^{2}+(c+1) \hat{\phi}_{U} .
$$

Comparando-se (3.9), para $\hat{\phi}_{U} \in[0,1]$, com (3.14) as constantes $a, b$ e $c$ são determinadas, e o limitador resultante é

$$
\psi\left(r_{f}\right)=-2 \alpha \hat{\phi}_{U}^{3}+(2 \alpha-2) \hat{\phi}_{U}^{2}+(-0.5 \alpha+3) \hat{\phi}_{U},
$$

ou utilizando-se (3.12) obtém-se

$$
\psi\left(r_{f}\right)=\frac{(-0.5 \alpha+1) r_{f}^{3}+(\alpha+4) r_{f}^{2}+(-0.5 \alpha+3) r_{f}}{\left(1+r_{f}\right)^{3}}, \quad r_{f} \geq 0 .
$$


Em resumo, o limitador para o esquema TOPUS é dado por

$$
\psi\left(r_{f}\right)=\left\{\begin{array}{l}
\frac{(-0.5 \alpha+1) r_{f}^{3}+(\alpha+4) r_{f}^{2}+(-0.5 \alpha+3) r_{f}}{\left(1+r_{f}\right)^{3}}, \quad r_{f} \geq 0, \\
0, \quad r_{f}<0 .
\end{array}\right.
$$

A Figura 3.3b mostra no plano $\psi-r_{f}$ o limitador do esquema TOPUS para $\alpha=-2, \alpha=0$ e $\alpha=2$. Nota-se que o limitador está inteiramente na região TVD de Sweby [139] para $\alpha=2$.

Para a implementação computacional, reescreve-se esse limitador como

$$
\psi\left(r_{f}\right)=\frac{0.5\left(\left|r_{f}\right|+r_{f}\right)\left[(-0.5 \alpha+1) r_{f}^{2}+(\alpha+4) r_{f}+(-0.5 \alpha+3)\right]}{\left(1+\left|r_{f}\right|\right)^{3}} .
$$

Derivando a equação (3.17), para $r_{f} \geq 0$, em relação a $r_{f}$ tem-se

$$
\psi^{\prime}\left(r_{f}\right)=\frac{(-2.5 \alpha-1) r_{f}^{2}+(3 \alpha+2) r_{f}-0.5 \alpha+3}{\left(1+r_{f}\right)^{4}},
$$

Nota-se que o TOPUS com $\alpha=2$ satisfaz uma restrição do princípio de monotonicidade de Sweby [139], isto é, $\psi^{\prime}(0)=2$. Além disso, o TOPUS é monotônico de segunda ordem [139], pois o seu limitador de fluxo satisfaz $\psi(1)=1$. De fato, percebe-se que o limitador (3.17) satisfaz

$$
\psi(1)=\frac{(-0.5 \alpha+1)+(\alpha+4)+(-0.5 \alpha+3)}{(1+1)^{3}}=1 .
$$

Ainda mais, de acordo com Zijlema [168] o limitador (3.17) satisfaz $\psi^{\prime}(1)=0.25$, que é a condição para alcançar terceira ordem de precisão. Na notação mais usual, o esquema TOPUS é

$$
\psi\left(r_{f}\right)=\max \left\{0, \frac{0.5\left(\left|r_{f}\right|+r_{f}\right)\left[(-0.5 \alpha+1) r_{f}^{2}+(\alpha+4) r_{f}+(-0.5 \alpha+3)\right]}{\left(1+\left|r_{f}\right|\right)^{3}}\right\} .
$$

Abaixo, estão listados alguns exemplos de limitadores de fluxo de esquemas "upwind" de alta resolução obtidos da mesma forma que o limitador TOPUS. Outros exemplos serão citados nos capítulos 5 e 6.

- ADBQUICKEST

$$
\psi\left(r_{f}\right)=\max \left\{0, \min \left[2 r_{f}(1-\theta), \frac{2+\theta^{2}-3|\theta|+\left(1-\theta^{2}\right) r_{f}}{3}, 2(1-\theta)\right]\right\},
$$

\section{- CUBISTA}

$$
\psi\left(r_{f}\right)=\max \left\{0, \min \left[2 r_{f}\left(1-C_{1}\right), 0.75+0.25 r_{f}, 2\left(1-C_{2}\right)\right]\right\},
$$

em que $C_{1}=C_{2}=0.25$; 
- VONOS

$$
\psi\left(r_{f}\right)=\max \left\{0, \min \left[r_{f}, 0.75+0.25 r_{f}, 18 r_{f}, 2\right]\right\}
$$

- WACEB

$$
\psi\left(r_{f}\right)=\max \left\{0, \min \left[2 r_{f}, 0.75+0.25 r_{f}, 2\right]\right\}
$$

(a)

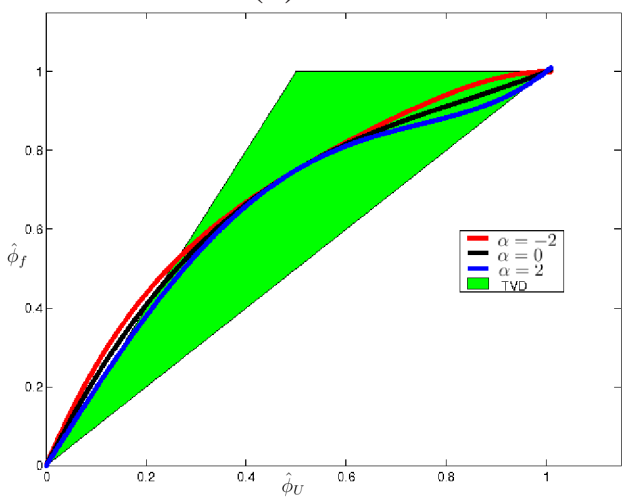

(b)

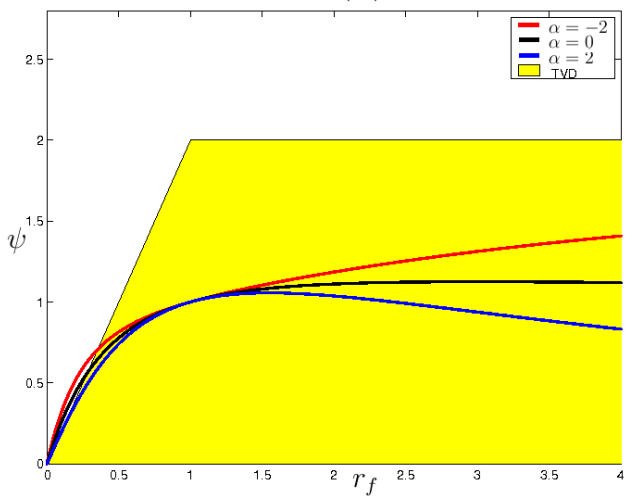

Figura 3.3: Curvas características do esquema TOPUS: (a) região TVD de Harten [61], (b) limitador na região TVD de Sweby [139].

\subsection{Esquema ALUS}

O interesse em desenvolver outro esquema TVD, que possua o número de Courant $(|\theta| \leq 1)$ em sua formulação (veja [83]) e seja matematicamente mais simples (sem perder em exatidão) que o esquema ADBQUICKEST de Ferreira et al. [49], motivou a derivação do esquema ALUS ${ }^{2}$.

Em analogia ao esquema ADBQUICKEST, a estratégia para obter o esquema ALUS é definir o limitador $\psi$ como

$$
\begin{cases}\psi\left(r_{f}\right)=0, & \operatorname{para} r_{f} \leq 0, \\ 0 \leq \psi\left(r_{f}\right) \leq \min \left\{2 r_{f}, 1-|\theta|\right\}, & \text { para } r_{f}>0,\end{cases}
$$

o qual pode ser reescrito na forma mais utilizada na literatura (veja, por exemplo, $[3,154]$ ) como

$$
\psi\left(r_{f}\right)=\max \left\{0, \min \left[2 r_{f}, 1-|\theta|\right]\right\} .
$$

Da mesma forma como feito para o esquema TOPUS na seção 3.2, o limitador (3.21) satisfaz as restrições TVD de Sweby [139], isto é,

$$
\begin{cases}\psi\left(r_{f}\right)=0, & \text { para } r_{f} \leq 0, \\ 0 \leq \psi\left(r_{f}\right) \leq \min \left\{2 r_{f}, 2\right\}, & \text { para } r_{f}>0 .\end{cases}
$$

\footnotetext{
${ }^{2}$ No contexto bíblico, ALUS é um local, onde os israelitas acamparam durante as suas vagueações pelo deserto.
} 
(a)

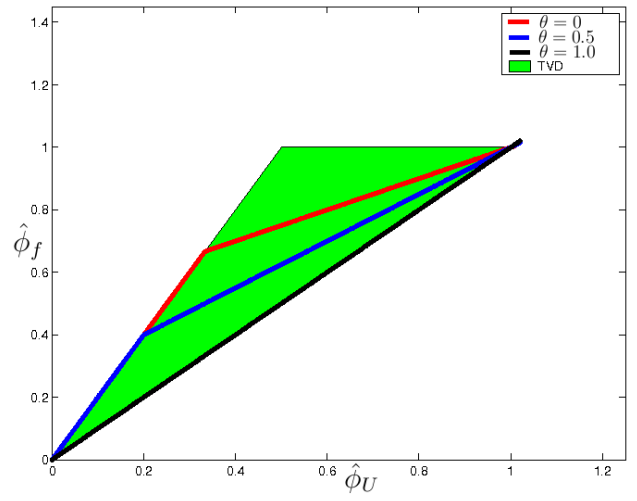

(b)

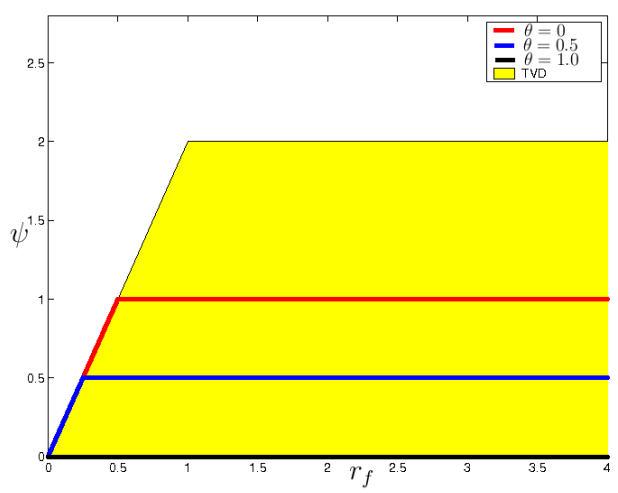

Figura 3.4: Curvas características do esquema ALUS: (a) região TVD de Harten [61], (b) limitador na região TVD de Sweby [139].

Na Figura 3.4, os casos a e b mostram, respectivamente, o esquema ALUS na região TVD de Harten [61] e o seu limitador na região TVD de Sweby [139].

Substituindo (3.21) em (3.11) e usando (3.12) obtém-se o esquema ALUS em NV

$$
\hat{\phi}_{f}= \begin{cases}2 \hat{\phi}_{U}, & \hat{\phi}_{U} \in\left[0, \lambda_{a}\right], \\ 0.5\left[(1+|\theta|) \hat{\phi}_{U}+(1-|\theta|)\right], & \hat{\phi}_{U} \in\left(\lambda_{a}, 1\right], \\ \hat{\phi}_{U}, & \hat{\phi}_{U} \notin[0,1] .\end{cases}
$$

em que $\lambda_{a}$ é a intersecção das retas $0.5\left[(1+|\theta|) \hat{\phi}_{U}+(1-|\theta|)\right]$ e $2 \hat{\phi}_{U}$, que é dada por

$$
\lambda_{a}=\frac{1-|\theta|}{3-|\theta|}
$$

A formulação do ALUS em variáveis não normalizadas, obtida do mesmo modo para o esquema TOPUS, é dada por

$$
\phi_{f}= \begin{cases}2 \phi_{U}-\phi_{R}, & \hat{\phi}_{U} \in\left[0, \lambda_{a}\right], \\ 0.5\left[(1+|\theta|) \phi_{U}+(1-|\theta|) \phi_{D}\right], & \hat{\phi}_{U} \in\left(\lambda_{a}, 1\right], \\ \phi_{U}, & \hat{\phi}_{U} \notin[0,1] .\end{cases}
$$

Outra forma de se reescrever o limitador (3.21) é utilizar as equações (3.12), (3.11) e (3.24), isto é,

$$
\psi\left(r_{f}\right)=\left\{\begin{array}{l}
2 r_{f}, \quad r_{f} \in\left[0, \frac{1-|\theta|}{2}\right], \\
1-|\theta|, \quad r_{f} \in\left(\frac{1-|\theta|}{2},+\infty\right), \\
0, \quad r_{f} \in(-\infty, 0) .
\end{array}\right.
$$




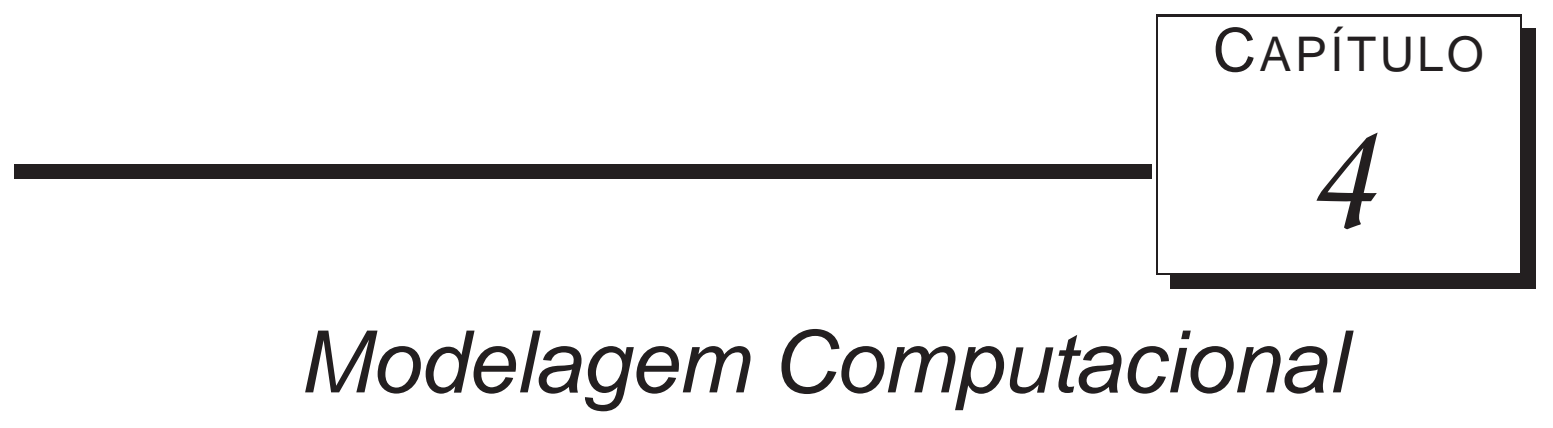

Neste capítulo é discutida a discretização dos termos convectivos das equações de transporte (2.1), (2.4), (2.16), (2.18), (2.22), (2.24), (2.34), (2.38), (2.39), (2.55) e (2.56). Também são apresentados algoritmos para a solução computacional das equações de Navier-Stokes (instantâneas e médias).

As equações de advecção 1D, de convecção-difusão 1D, de Navier-Stokes (instantâneas e médias), da modelagem da turbulência e do modelo Oldroyd-B foram discretizadas pelo método de diferenças finitas [129]. No caso particular de escoamentos incompressíveis, foi utilizada uma malha deslocada ${ }^{1}$ ("staggered grid") [9] para a discretização das equações Navier-Stokes (com ou sem modelagem), e o sistema discreto de equações resultante foi implementado no sistema de simulação "Freeflow" de Castelo et al. [20] (ver também [17, 42, 126] para mais detalhes). Este ambiente computacional computa as equações de transporte e da continuidade baseado no método GENSMAC ("Generalized-Simplified-Marker-and-Cell”) [145, 146]. No caso de sistemas hiperbólicos (águas rasas 1D e de equações de Euler 1D/2D), o método de volumes finitos [82, 147] foi empregado para as discretizações. Para resolver as equações de águas rasas, o pacote computacional CLAWPACK ("Conservation LAW PACKage") de LeVeque [82] foi equipado com os esquemas ALUS e TOPUS. Para solucionar as equações de Euler 2D, o fluxo na face do volume é aproximado pelo método "upwind" tipo Godunov proposto por Roe [117] com reconstrução de variáveis [65] combinado com o limitador de fluxo do esquema TOPUS.

Para a marcha no tempo, foi utilizado em todas as equações dependentes do tempo o método de Euler explícito, com exceção na simulação dos escoamentos de Poiseuille e viscoelásticos onde se utilizou o método implícito de Cranck-Nicolson [129]. Para os termos difusivos e gradientes de pressão, diferenciação centrada de segunda ordem foi adotada.

Os termos convectivos (objeto deste capítulo) são aproximados pelos esquemas "upwind" de alta precisão ALUS e TOPUS.

\footnotetext{
${ }^{1}$ Neste tipo de malha, a pressão e as variáveis turbulentas $\kappa, \varepsilon$ e $\nu_{t}$ são aproximadas no centro da célula e as componentes da velocidade $u$ e $v$ nas faces $\left(i+\frac{1}{2}, j\right)$ e $\left(i, j+\frac{1}{2}\right)$, respectivamente.
} 


\subsection{Discretização dos Termos Convectivos}

Um representante típico para os termos convectivos das equações de transporte (2.1), (2.4), (2.16), (2.18), (2.22), (2.24), (2.34), (2.38), (2.39), (2.55) e (2.56) pode ser colocado na forma

$$
\left.\frac{\partial\left(u_{j} \phi\right)}{\partial x_{j}}\right|_{P}=-\left(\left.\frac{\partial(u \phi)}{\partial x}\right|_{P}+\left.\frac{\partial(v \phi)}{\partial y}\right|_{P}\right),
$$

em que $\phi$ é a variável convectada (por exemplo $u, v, \kappa$, $\varepsilon$, etc.) e $u_{j}$ a velocidade de convecção. O ponto $P$ em (4.1) representa a posição em que o termo convectivo é avaliado. Por exemplo, a Figura 4.1 ilustra esse ponto $P$ de avaliação bem como as posições $\mathrm{D}, \mathrm{R}$ e $\mathrm{U}$, e as faces $f$ e $g$ das células computacionais.

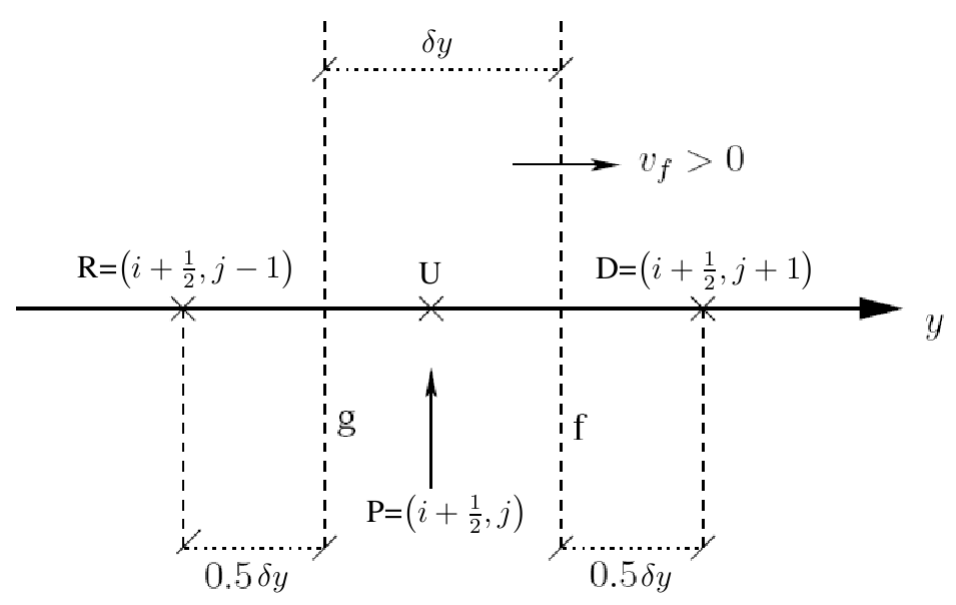

Figura 4.1: Célula computacional mostrando o ponto $P$ de discretização dos termos convectivos, seus vizinhos, as faces envolvidas $f$ e $g$ na aproximação e a variável convectada $\phi$ sendo transportada com velocidade $v_{f}$ na direção $y$.

As derivadas no lado direito de (4.1) são aproximadas no ponto $P$ por

$$
\begin{gathered}
\left.\frac{\partial(u \phi)}{\partial x}\right|_{P} \approx \frac{\left.(u \phi)\right|_{f}-\left.(u \phi)\right|_{g}}{\delta x}=\frac{u_{f} \phi_{f}-u_{g} \phi_{g}}{\delta x} \\
\left.\frac{\partial(v \phi)}{\partial y}\right|_{P} \approx \frac{\left.(v \phi)\right|_{f}-\left.(v \phi)\right|_{g}}{\delta y}=\frac{v_{f} \phi_{f}-v_{g} \phi_{g}}{\delta y}
\end{gathered}
$$

Por simplicidade e sem perda de generalidade, considera-se a variável $\phi=u$ em (4.3) transportada com velocidade $v_{f}$ na direção $y$ (ver Figura 4.1). Nesse caso, $P=\left(i+\frac{1}{2}, j\right), f=j+\frac{1}{2}$, $g=j-\frac{1}{2}$, e aquela derivada é estimada como 


$$
\left.\frac{\partial(v u)}{\partial y}\right|_{\left(i+\frac{1}{2}, j\right)}=\frac{\left(v_{f} u_{i+\frac{1}{2}, j+\frac{1}{2}}-v_{g} u_{i+\frac{1}{2}, j-\frac{1}{2}}\right)}{\delta y}
$$

em que $v_{f}$ e $v_{g}$ são aproximados, respectivamente, utilizando-se as médias

$$
\begin{aligned}
& v_{f}=v_{i+\frac{1}{2}, j+\frac{1}{2}}=\frac{\left(v_{i+1, j+\frac{1}{2}}+v_{i, j+\frac{1}{2}}\right)}{2}, \\
& v_{g}=v_{i+\frac{1}{2}, j-\frac{1}{2}}=\frac{\left(v_{i+1, j-\frac{1}{2}}+v_{i, j-\frac{1}{2}}\right)}{2} .
\end{aligned}
$$

Para completar a aproximação, os esquemas TOPUS (caso $\alpha=2$ ) e ALUS em variáveis não normalizadas são empregados. Esses esquemas estão definidos nas equações (3.10) e (3.25), respectivamente. Para tanto, os valores da propriedade transportada $u$ nas posições $\left(i+\frac{1}{2}, j+\frac{1}{2}\right)$ e $\left(i+\frac{1}{2}, j-\frac{1}{2}\right)$ são obtidos utilizando-se os pontos vizinhos $\mathrm{D}, \mathrm{R}$ e $\mathrm{U}$, os quais são definidos de acordo com a direção das velocidades de convecção (sinais de $v_{f}$ e $v_{g}$ ). Em resumo, tem-se:

- Aproximações para $u_{i+\frac{1}{2}, j+\frac{1}{2}}$ quando $v_{f} \geq 0$; nesse caso as posições $\mathrm{D}, \mathrm{R}$ e $\mathrm{U}$ assumem, respectivamente, os valores $\mathrm{D}=\left(i+\frac{1}{2}, j+1\right), \mathrm{R}=\left(i+\frac{1}{2}, j-1\right)$ e $\mathrm{U}=\left(i+\frac{1}{2}, j\right)$. $\mathrm{E}$ o valor $u_{i+\frac{1}{2}, j+\frac{1}{2}}$ usando-se os esquemas ALUS e TOPUS é obtido como:

- Esquema ALUS:

$$
u_{i+\frac{1}{2}, j+\frac{1}{2}}=\left\{\begin{array}{l}
2 u_{i+\frac{1}{2}, j}-u_{i+\frac{1}{2}, j-1}, \quad \hat{u}_{i+\frac{1}{2}, j} \in\left[0, \lambda_{a}\right], \\
0.5(1+|\theta|) u_{i+\frac{1}{2}, j}+0.5(1-|\theta|) u_{i+\frac{1}{2}, j+1}, \quad \hat{u}_{i+\frac{1}{2}, j} \in\left(\lambda_{a}, 1\right], \\
u_{i+\frac{1}{2}, j}, \quad \hat{u}_{i+\frac{1}{2}, j} \notin[0,1] .
\end{array}\right.
$$

- Esquema TOPUS:

$$
u_{i+\frac{1}{2}, j+\frac{1}{2}}=\left\{\begin{array}{l}
u_{i+\frac{1}{2}, j-1}+\left(u_{i+\frac{1}{2}, j+1}-u_{i+\frac{1}{2}, j-1}\right)\left(2 \hat{u}_{U}^{4}-3 \hat{u}_{U}^{3}+2 \hat{u}_{U}\right), \quad \hat{u}_{U} \in[0,1], \\
u_{i+\frac{1}{2}, j}, \quad \hat{u}_{U} \notin[0,1],
\end{array}\right.
$$

em que

$$
\hat{u}_{U}=\hat{u}_{i+\frac{1}{2}, j}=\frac{u_{i+\frac{1}{2}, j}-u_{i+\frac{1}{2}, j-1}}{u_{i+\frac{1}{2}, j+1}-u_{i+\frac{1}{2}, j-1}} .
$$

- Aproximações para $u_{i+\frac{1}{2}, j+\frac{1}{2}}$ quando $v_{f}<0: \mathrm{D}=\left(i+\frac{1}{2}, j\right), \mathrm{R}=\left(i+\frac{1}{2}, j+2\right)$ e $\mathrm{U}=\left(i+\frac{1}{2}, j+1\right)$

- Esquema ALUS:

$$
u_{i+\frac{1}{2}, j+\frac{1}{2}}=\left\{\begin{array}{l}
2 u_{i+\frac{1}{2}, j+1}-u_{i+\frac{1}{2}, j+2}, \quad \hat{u}_{i+\frac{1}{2}, j+1} \in\left[0, \lambda_{a}\right], \\
0.5(1+|\theta|) u_{i+\frac{1}{2}, j+1}+0.5(1-|\theta|) u_{i+\frac{1}{2}, j}, \quad \hat{u}_{i+\frac{1}{2}, j+1} \in\left(\lambda_{a}, 1\right], \\
u_{i+\frac{1}{2}, j+1}, \quad \hat{u}_{i+\frac{1}{2}, j+1} \notin[0,1] .
\end{array}\right.
$$


- Esquema TOPUS:

$$
u_{i+\frac{1}{2}, j+\frac{1}{2}}=\left\{\begin{array}{l}
u_{i+\frac{1}{2}, j+2}+\left(u_{i+\frac{1}{2}, j}-u_{i+\frac{1}{2}, j+2}\right)\left(2 \hat{u}_{U}^{4}-3 \hat{u}_{U}^{3}+2 \hat{u}_{U}\right), \quad \hat{u}_{U} \in[0,1], \\
u_{i+\frac{1}{2}, j+1}, \quad \hat{u}_{U} \notin[0,1],
\end{array}\right.
$$

em que

$$
\hat{u}_{U}=\hat{u}_{i+\frac{1}{2}, j+1}=\frac{u_{i+\frac{1}{2}, j+1}-u_{i+\frac{1}{2}, j+2}}{u_{i+\frac{1}{2}, j}-u_{i+\frac{1}{2}, j+2}} .
$$

- Aproximações para $u_{i+\frac{1}{2}, j-\frac{1}{2}}$ quando $v_{g} \geq 0: \mathrm{D}=\left(i+\frac{1}{2}, j\right), \mathrm{R}=\left(i+\frac{1}{2}, j-2\right)$ e $\mathrm{U}=\left(i+\frac{1}{2}, j-1\right)$

- Esquema ALUS:

$$
u_{i+\frac{1}{2}, j-\frac{1}{2}}=\left\{\begin{array}{l}
2 u_{i+\frac{1}{2}, j-1}-u_{i+\frac{1}{2}, j-2}, \quad \hat{u}_{i+\frac{1}{2}, j-1} \in\left[0, \lambda_{a}\right], \\
0.5(1+|\theta|) u_{i+\frac{1}{2}, j-1}+0.5(1-|\theta|) u_{i+\frac{1}{2}, j}, \quad \hat{u}_{i+\frac{1}{2}, j-1} \in\left(\lambda_{a}, 1\right], \\
u_{i+\frac{1}{2}, j-1}, \quad \hat{u}_{i+\frac{1}{2}, j-1} \notin[0,1] .
\end{array}\right.
$$

- Esquema TOPUS:

$$
u_{i+\frac{1}{2}, j-\frac{1}{2}}=\left\{\begin{array}{l}
u_{i+\frac{1}{2}, j-2}+\left(u_{i+\frac{1}{2}, j}-u_{i+\frac{1}{2}, j-2}\right)\left(2 \hat{u}_{U}^{4}-3 \hat{u}_{U}^{3}+2 \hat{u}_{U}\right), \quad \hat{u}_{U} \in[0,1], \\
u_{i+\frac{1}{2}, j-1}, \quad \hat{u}_{U} \notin[0,1],
\end{array}\right.
$$

em que

$$
\hat{u}_{U}=\hat{u}_{i+\frac{1}{2}, j-1}=\frac{u_{i+\frac{1}{2}, j-1}-u_{i+\frac{1}{2}, j-2}}{u_{i+\frac{1}{2}, j}-u_{i+\frac{1}{2}, j-2}} .
$$

- Aproximações para $u_{i+\frac{1}{2}, j-\frac{1}{2}}$ quando $v_{g}<0: \mathrm{D}=\left(i+\frac{1}{2}, j-1\right), \mathrm{R}=\left(i+\frac{1}{2}, j+1\right), \mathrm{U}=\left(i+\frac{1}{2}, j\right)$

- Esquema ALUS:

$$
u_{i+\frac{1}{2}, j-\frac{1}{2}}=\left\{\begin{array}{l}
2 u_{i+\frac{1}{2}, j}-u_{i+\frac{1}{2}, j+1}, \quad \hat{u}_{i+\frac{1}{2}, j} \in\left[0, \lambda_{a}\right], \\
0.5(1+|\theta|) u_{i+\frac{1}{2}, j}+0.5(1-|\theta|) u_{i+\frac{1}{2}, j-1}, \quad \hat{u}_{i+\frac{1}{2}, j} \in\left(\lambda_{a}, 1\right], \\
u_{i+\frac{1}{2}, j}, \quad \hat{u}_{i+\frac{1}{2}, j} \notin[0,1] .
\end{array}\right.
$$

- Esquema TOPUS:

$u_{i+\frac{1}{2}, j-\frac{1}{2}}=\left\{\begin{array}{l}u_{i+\frac{1}{2}, j+1}+\left(u_{i+\frac{1}{2}, j-1}-u_{i+\frac{1}{2}, j+1}\right)\left(2 \hat{u}_{U}^{4}-3 \hat{u}_{U}^{3}+2 \hat{u}_{U}\right), \quad \hat{u}_{U} \in[0,1], \\ u_{i+\frac{1}{2}, j}, \quad \hat{u}_{U} \notin[0,1],\end{array}\right.$

em que

$$
\hat{u}_{U}=\hat{u}_{i+\frac{1}{2}, j}=\frac{u_{i+\frac{1}{2}, j}-u_{i+\frac{1}{2}, j+1}}{u_{i+\frac{1}{2}, j-1}-u_{i+\frac{1}{2}, j+1}} .
$$


Outro exemplo de aproximação "upwind" usando os esquemas ALUS e TOPUS é ilustrado no caso em que a variável transportada, com velocidade $u$ na direção $x$, é a variável turbulenta $\kappa$. Nesse caso, a aproximação para a derivada convectiva correspondente é dada por

$$
\left.\frac{\partial(u \kappa)}{\partial x}\right|_{P}=\frac{\left.(u \kappa)\right|_{f}-\left.(u \kappa)\right|_{g}}{\delta x}
$$

em que $P, f$ e $g$ assumem, respectivamente, as posições $(i, j), i+\frac{1}{2}$ e $i-\frac{1}{2}$. Desta forma, a equação (4.6) torna-se

$$
\left.\frac{\partial(\kappa u)}{\partial x}\right|_{(i, j)}=\frac{u_{i+\frac{1}{2}, j} \kappa_{i+\frac{1}{2}, j}-u_{i-\frac{1}{2}, j} \kappa_{i-\frac{1}{2}, j}}{\delta x},
$$

Aproximações para a grandeza $\kappa$ na equação (4.7) são implementadas usando-se os esquemas ALUS e TOPUS como seguem:

- Aproximações para $\kappa_{i+\frac{1}{2}, j}$ quando $u_{i+\frac{1}{2}, j} \geq 0: \mathrm{D}=(i+1, j), \mathrm{R}=(i-1, j)$ e $\mathrm{U}=(i, j)$

- Esquema ALUS:

$$
\kappa_{i+\frac{1}{2}, j}=\left\{\begin{array}{l}
2 \kappa_{i, j}-\kappa_{i-1, j}, \quad \hat{\kappa}_{i, j} \in\left[0, \lambda_{a}\right], \\
0.5(1+|\theta|) \kappa_{i, j}+0.5(1-|\theta|) \kappa_{i+1, j}, \quad \hat{\kappa}_{i, j} \in\left(\lambda_{a}, 1\right], \\
\kappa_{i, j}, \quad \hat{\kappa}_{i, j} \notin[0,1] .
\end{array}\right.
$$

- Esquema TOPUS:

$$
\kappa_{i+\frac{1}{2}, j}=\left\{\begin{array}{l}
\kappa_{i-1, j}+\left(\kappa_{i+1, j}-\kappa_{i-1, j}\right)\left(2 \hat{\kappa}_{U}^{4}-3 \hat{\kappa}_{U}^{3}+\hat{\kappa}_{U}\right), \quad \hat{\kappa}_{U} \notin[0,1], \\
\kappa_{i, j}, \quad \hat{\kappa}_{U} \notin[0,1],
\end{array}\right.
$$

em que

$$
\hat{\kappa}_{U}=\kappa_{i, j}=\frac{\kappa_{i, j}-\kappa_{i-1, j}}{\kappa_{i+1, j}-\kappa_{i-1, j}} .
$$

- Aproximações para $\kappa_{i+\frac{1}{2}, j}$ quando $u_{i+\frac{1}{2}, j}<0: \mathrm{D}=(i, j), \mathrm{R}=(i+2, j)$ e $\mathrm{U}=(i+1, j)$,

- Esquema ALUS:

$$
\kappa_{i+\frac{1}{2}, j}=\left\{\begin{array}{l}
2 \kappa_{i+1, j}-\kappa_{i+2, j}, \quad \hat{\kappa}_{i+1, j} \in\left[0, \lambda_{a}\right], \\
0.5(1+|\theta|) \kappa_{i+1, j}+0.5(1-|\theta|) \kappa_{i, j}, \quad \hat{\kappa}_{i+1, j} \in\left(\lambda_{a}, 1\right], \\
\kappa_{i+1, j}, \quad \hat{\kappa}_{i+1, j} \notin[0,1] .
\end{array}\right.
$$


- Esquema TOPUS:

$$
\kappa_{i+\frac{1}{2}, j}=\left\{\begin{array}{l}
\kappa_{i+2, j}+\left(\kappa_{i, j}-\kappa_{i+2, j}\right)\left(2 \hat{\kappa}_{U}^{4}-3 \hat{\kappa}_{U}^{3}+\hat{\kappa}_{U}\right), \quad \hat{\kappa}_{U} \in[0,1], \\
\kappa_{i+1, j}, \quad \hat{\kappa}_{U} \notin[0,1],
\end{array}\right.
$$

em que

$$
\hat{\kappa}_{U}=\hat{\kappa}_{i+1, j}=\frac{\kappa_{i+1, j}-\kappa_{i+2, j}}{\kappa_{i, j}-\kappa_{i+2, j}} .
$$

- Aproximações para $\kappa_{i-\frac{1}{2}, j}$ quando $u_{i-\frac{1}{2}, j} \geq 0: \mathrm{D}=(i, j), \mathrm{R}=(i-2, j)$ e $\mathrm{U}=(i-1, j)$

- Esquema ALUS:

$$
\kappa_{i-\frac{1}{2}, j}=\left\{\begin{array}{l}
2 \kappa_{i-1, j}-\kappa_{i-2, j}, \quad \hat{\kappa}_{i-1, j} \in\left[0, \lambda_{a}\right], \\
0.5(1+|\theta|) \kappa_{i-1, j}+0.5(1-|\theta|) \kappa_{i, j}, \quad \hat{\kappa}_{i-1, j} \in\left(\lambda_{a}, 1\right], \\
\kappa_{i-1, j}, \quad \hat{\kappa}_{i-1, j} \notin[0,1] .
\end{array}\right.
$$

- Esquema TOPUS:

em que

$$
\kappa_{i-\frac{1}{2}, j}=\left\{\begin{array}{l}
\kappa_{i-2, j}+\left(\kappa_{i, j}-\kappa_{i-2, j}\right)\left(2 \hat{\kappa}_{U}^{4}-3 \hat{\kappa}_{U}^{3}+\hat{\kappa}_{U}\right), \quad \hat{\kappa}_{U} \in[0,1], \\
\kappa_{i-1, j}, \quad \hat{\kappa}_{U} \notin[0,1],
\end{array}\right.
$$

$$
\hat{\kappa}_{U}=\hat{\kappa}_{i-1, j}=\frac{\kappa_{i-1, j}-\kappa_{i-2, j}}{\kappa_{i, j}-\kappa_{i-2, j}} .
$$

- Aproximações para $\kappa_{i-\frac{1}{2}, j}$ quando $u_{i-\frac{1}{2}, j}<0$ : $\mathrm{D}=(i-1, j), \mathrm{R}=(i+1, j)$ e $\mathrm{U}=(i, j)$

- Esquema ALUS:

$$
\kappa_{i-\frac{1}{2}, j}=\left\{\begin{array}{l}
2 \kappa_{i, j}-\kappa_{i+1, j}, \quad \hat{\kappa}_{i, j} \in\left[0, \lambda_{a}\right], \\
0.5(1+|\theta|) \kappa_{i, j}+0.5(1-|\theta|) \kappa_{i-1, j}, \quad \hat{\kappa}_{i, j} \in\left(\lambda_{a}, 1\right], \\
\kappa_{i, j}, \quad \hat{\kappa}_{i, j} \notin[0,1] .
\end{array}\right.
$$

- Esquema TOPUS:

$$
\kappa_{i-\frac{1}{2}, j}=\left\{\begin{array}{l}
\kappa_{i+1, j}+\left(\kappa_{i-1, j}-\kappa_{i+1, j}\right)\left(2 \hat{\kappa}_{U}^{4}-3 \hat{\kappa}_{U}^{3}+\hat{\kappa}_{U}\right), \quad \hat{\kappa}_{U} \in[0,1], \\
\kappa_{i, j}, \quad \hat{\kappa}_{i, j} \notin[0,1],
\end{array}\right.
$$

em que

$$
\hat{\kappa}_{U}=\hat{\kappa}_{i, j}=\frac{\kappa_{i, j}-\kappa_{i+1, j}}{\kappa_{i-1, j}-\kappa_{i+1, j}} .
$$

As outras derivadas convectivas nas equações de conservação são implementadas de maneira análoga às aproximações dos termos não-lineares nas equações (4.4) e (4.7). É importante observar que os esquemas ALUS e TOPUS fazem uso de três pontos vizinhos (D, U, e R) para estimar uma variável convectada. Portanto, podem ocorrer valores fora do domínio de solução para as células computacionais que estão próximas às fronteiras. Neste caso, o esquema FOU de primeira ordem é empregado. 


\subsection{Algoritmo para Simulação de Escoamentos Incom- pressíveis Laminares}

Os passos de um ciclo computacional do algoritmo são descritos na seqüência para resolver as EDPs (2.24) e (2.25), e o método de cálculo é baseado no método da projeção de Chorin [23]. Considera-se que no tempo $t_{0}$ as condições iniciais e de contorno são conhecidas para o campo de velocidade e pressão [146]. O campo de velocidade no tempo $t=t_{0}+\delta t$ é calculado pela sequiência de passos:

Passo 1: Atualizam-se as condições de contorno nas regiões de entrada e saída de fluido e nas paredes rígidas. Calcula-se o campo de velocidade na superfície livre usando-se as equações (2.30) e (2.31). A pressão na superfície livre é determinada utilizando-se a equação (2.32) [146];

Passo 2: Calcula-se o campo de velocidade intermediária $\tilde{u}_{i}$ por meio de

$$
\tilde{u}_{i}=u_{i}+\delta t\left\{-\frac{\partial\left(u_{i} u_{j}\right)}{\partial x_{j}}-\frac{\partial \tilde{p}}{\partial x_{i}}+\frac{1}{R e} \frac{\partial}{\partial x_{j}}\left(\frac{\partial u_{i}}{\partial x_{j}}\right)+\frac{1}{F r^{2}} g_{i}\right\}, \quad i=1,2,3,
$$

em que $\tilde{p}$ é uma pressão arbitrária satisfazendo a condição correta para a pressão na superfície livre;

Passo 3: Resolve-se a equação de Poisson para o potencial auxiliar $\psi$

$$
\frac{\partial}{\partial x_{i}}\left(\frac{\partial \psi}{\partial x_{i}}\right)=\left(\frac{\partial \tilde{u}_{i}}{\partial x_{i}}\right), \quad i=1,2,3
$$

$\operatorname{com} \psi=0$ nas regiões de saída e superfície livre, e com $\frac{\partial \psi}{\partial n}=0$ nos contornos de entrada e rígido (ver, por exemplo, [4]). Neste trabalho, o método gradiente conjugado [64] foi usado para resolver o sistema de equações lineares (4.9);

Passo 4: Atualiza-se o campo de velocidade por (ver detalhes em [34])

$$
u_{i}=\tilde{u}_{i}-\frac{\partial \psi}{\partial x_{i}}, \quad i=1,2,3
$$

Passo 5: Atualiza-se a pressão da seguinte maneira (para detalhes veja [146])

$$
p=\tilde{p}+\frac{\psi}{\delta t}
$$

Passo 6: Calculam-se, por Euler explícito [11], as posições das partículas marcadoras representando o fluido por meio do sistema de Equações Diferenciais Ordinárias (EDOs)

$$
\frac{d x_{i}}{d t}=u_{i}, \quad i=1,2,3
$$

E volta-se ao Passo 1 para dar início ao próximo ciclo computacional. 


\subsection{Algoritmo para Simulação de Escoamentos Incom- pressíveis Turbulentos}

Nesta seção são apresentados os passos do algoritmo para o cálculo das variáveis $u, v, \kappa \mathrm{e} \varepsilon$ nas EDPs (2.34), (2.35), (2.38) e (2.39). Como na seção anterior, considera-se que no instante $t_{0}$ as variáveis dependentes são conhecidas e as condições de fronteira associadas estão especificadas [146]. As equações médias de Navier-Stokes e as equações que descrevem as características da turbulência são resolvidas de maneira desacoplada (da mesma maneira como foi feito na seção anterior): as equações médias do movimento e continuidade são resolvidas primeiro, mantendo-se todas as grandezas turbulentas "congeladas"; a seguir, as equações para as variáveis turbulentas são resolvidas [42]. A seqüência de um ciclo computacional é descrita como segue:

Passo 1: Com a viscosidade turbulenta $\nu_{t}$ conhecida no tempo inicial $t_{0}$, ou num ciclo prévio, computa-se um campo de velocidade tentativo $\tilde{u}_{i}$ em $t=t_{0}+\delta t$, por meio de

$$
\tilde{u}_{i}=u_{i}+\delta t\left\{-\frac{\partial\left(u_{i} u_{j}\right)}{\partial x_{j}}-\frac{\partial \tilde{p}}{\partial x_{i}}+\frac{1}{R e} \frac{\partial}{\partial x_{j}}\left(\frac{\partial u_{i}}{\partial x_{j}}\right)+\frac{1}{F r^{2}} g_{i}-\frac{1}{R e} \frac{\partial\left(\overline{u_{i} u_{j}}\right)}{\partial x_{j}}\right\}, \quad i=1,2 .
$$

Neste estudo, o tensor de tensão de Reynolds $\overline{u_{i} u_{j}}$ é calculado pelo modelo $\kappa-\varepsilon$ padrão (2.36) ou pelo modelo RSAEM (2.51);

Passo 2: Resolve-se a equação de Poisson para o potencial auxiliar $\psi$

$$
\frac{\partial}{\partial x_{i}}\left(\frac{\partial \psi}{\partial x_{i}}\right)=\left(\frac{\partial \tilde{u}_{i}}{\partial x_{i}}\right), \quad i=1,2,
$$

utilizando-se as mesmas condições do Passo 3 do algoritmo descrito na seção 4.2;

Passo 3: Atualiza-se o campo de velocidade por (ver [34])

$$
u_{i}=\tilde{u}-\frac{\partial \psi}{\partial x_{i}}, \quad i=1,2
$$

Passo 4: Atualiza-se a pressão a partir da equação (ver [146])

$$
p=\tilde{p}+\frac{\psi}{\delta t}
$$

Passo 5: Calcula-se energia cinética turbulenta $\kappa$ como

$$
\kappa^{(n+1)}=\kappa^{(n)}+\delta t\left\{-\frac{\partial\left(\kappa u_{j}\right)}{\partial x_{j}}+\frac{1}{R e} \frac{\partial}{\partial x_{j}}\left(\left(1+\frac{\nu_{t}}{\sigma_{\kappa}}\right) \frac{\partial \kappa}{\partial x_{j}}\right)+\nu_{t} D_{i j} \frac{\partial u_{i}}{\partial x_{j}}-\varepsilon\right\}, \quad i=1,2,
$$

em que $D_{i j}$ é definido em (2.37) e $(n+1)$ denota o passo atual;

Passo 6: Obtém-se a dissipação de energia $\varepsilon$ por

$$
\varepsilon^{(n+1)}=\varepsilon^{(n)}+\delta t\left\{-\frac{\partial\left(\varepsilon u_{j}\right)}{\partial x_{j}}+\frac{1}{R e} \frac{\partial}{\partial x_{j}}\left(\left(1+\frac{\nu_{t}}{\sigma_{\varepsilon}}\right) \frac{\partial \varepsilon}{\partial x_{j}}\right)+\frac{1}{T_{t}}\left(C_{1 \varepsilon} \nu_{t} D_{i j} \frac{\partial u_{i}}{\partial x_{j}}-C_{2 \varepsilon} \varepsilon\right)\right\} ;
$$

Passo 7: Calcula-se a viscosidade turbulenta $\nu_{t}$ por (2.41) ou (2.52), dependendo do modelo de turbulência escolhido; 
Passo 8: Determinam-se as posições das partículas marcadoras através da aplicação do método de Euler [11] ao sistema de EDOs

$$
\frac{d x_{i}}{d t}=u_{i}, \quad i=1,2
$$

Passo 9: Atualizam-se as condições de contorno necessárias para o próximo ciclo. 


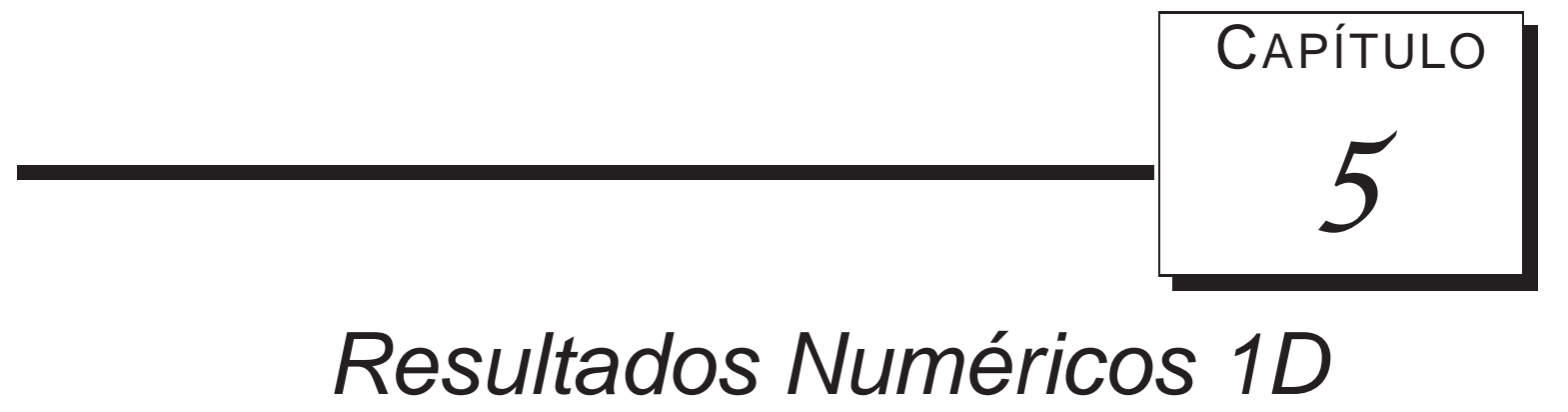

Com o objetivo de demonstrar o comportamento, a validade, a flexibilidade e a robustez dos esquemas ALUS e TOPUS, neste capítulo, um número extensivo de resultados numéricos 1D são apresentados. Comparações com soluções exatas e resultados de outros esquemas "upwind" consagrados na literatura são também apresentadas.

\subsection{Advecção Linear}

Aqui, os resultados da solução numérica de (2.1), com condições iniciais e tempo de simulação distintos, são considerados. Esses dados foram publicados em anais de eventos e periódicos (ver $[48,105,110,111,115])$. As condições de contorno em (2.3) são $u_{L}=u_{R}=0$. Os seguintes esquemas foram adotados para simular este problema: ADBQUICKEST, ALUS, FOU, Lax-Wendroff [77], TOPUS e VONOS.

- Caso 1: considera-se a equação (2.1) com $a=1, x \in[0,2]$ e condição inicial [74]

$$
u_{0}(x)= \begin{cases}\exp \left(-\log 50\left(\frac{x-0.15}{0.05}\right)^{2}\right), & x \in[0,0.2), \\ 1, & x \in(0.3,0.4), \\ 20 x-10, & x \in(0.5,0.55), \\ -20 x+12, & x \in[0.55,0.66), \\ \sqrt{1-\left(\frac{x-0.75}{0.05}\right)^{2}}, & x \in(0.7,0.8), \\ 0, & x \in I,\end{cases}
$$

em que $I=[0.2,0.3] \cup[0.4,0.5] \cup[0.66,0.7] \cup[0.8,2]$.

Neste caso foram adotados uma malha de $N=400$ células $\left(\delta x=5 \times 10^{-3}\right)$, espaçamentos temporais $\delta t=2.5 \times 10^{-4}$ e $\delta t=2.5 \times 10^{-3}$, e tempo de simulação $t=1.0$. Os números de Courant considerados nas simulações foram $\theta=0.05$ e $\theta=0.5(\theta=(a \delta t) / \delta x)$. Os resultados numéricos usando os vários esquemas "upwind" mencionados acima estão mostrados nas Figuras 5.1 e 5.2. Como se pode ver a partir dessa figura, os esquemas ALUS 
e FOU apresentaram dissipação numérica evidente, com o ALUS um pouco menos dissipativo. Observa-se também por essas figuras que os esquemas Lax-Wendroff e VONOS sofreram oscilações indesejáveis. Por outro lado, os esquemas ADBQUICKEST e TOPUS forneceram, quando comparados com a solução exata [92], resultados de boa qualidade. Diferentemente do ADBQUICKEST, nota-se na Figura 5.2, que o TOPUS capturou bem os picos presentes na solução do problema quando se utilizou $\theta=0.5$.

- Caso 2: com o objetivo de testar o comportamento dos esquemas ALUS e TOPUS num problema mais difícil ("W-shape"), proposto por Wei e Gu [156], adota-se a equação (2.1) com $a=1, x \in[-1,1]$ e condição inicial

$$
u_{0}(x)= \begin{cases}1, & x \in[0,0.2] \\ 4 x-0.6, & x \in(0.2,0.4] \\ -4 x+2.6, & x \in(0.4,0.6] \\ 1, & x \in(0.6,0.8] \\ 0, & x \in S=[-1,0) \cup(0.8,1] .\end{cases}
$$

Neste caso, foram adotadas as mesmas condições (malha, números de Courant, espaçamentos temporais) das simulações do caso 1. O tempo final de simulação foi $t=0.25$. Os resultados numéricos usando os vários esquemas "upwind" mencionados anteriormente estão apresentados nas Figuras 5.3 e 5.4. Neste caso, os comportamentos dos esquemas foram similares aos observados no Caso 1. Porém, os picos e vales foram bem resolvidos com os esquemas ADBQUICKEST, ALUS e TOPUS, sem apresentar qualquer vestígio de oscilação não física.

- Caso 3: neste caso, utiliza-se a equação (2.1) com $a=1, x \in[-1,1]$ e condição inicial [62]

$$
u_{0}(x)= \begin{cases}-x \sin \left(\frac{3 \pi x^{2}}{2}\right), & x \in\left[-1,-\frac{1}{3}\right] \\ |\sin (2 \pi x)|, & x \in\left(-\frac{1}{3}, \frac{1}{3}\right) \\ 2 x-1-\frac{1}{6} \sin (3 \pi x), & x \in\left[\frac{1}{3}, 1\right]\end{cases}
$$

Para a simulação desse problema altamente descontínuo, foram consideradas as mesmas condições (malha, números de Courant, espaçamentos temporais) do caso 1. O tempo de simulação $t=0.125$ foi adotado. Nas Figuras 5.5 e 5.6 são apresentadas as comparações entre as soluções exata e numéricas. Pode-se inferir claramente dessas comparações as mesmas conclusões daquelas extraídas nos casos anteriores. Cabe salientar que o método numérico de diferenças finitas explícito combinado com as estratégias "upwind" ALUS e TOPUS forneceu soluções satisfatórias para ambos os números de Courant utilizados nos casos delineados nesta seção. 

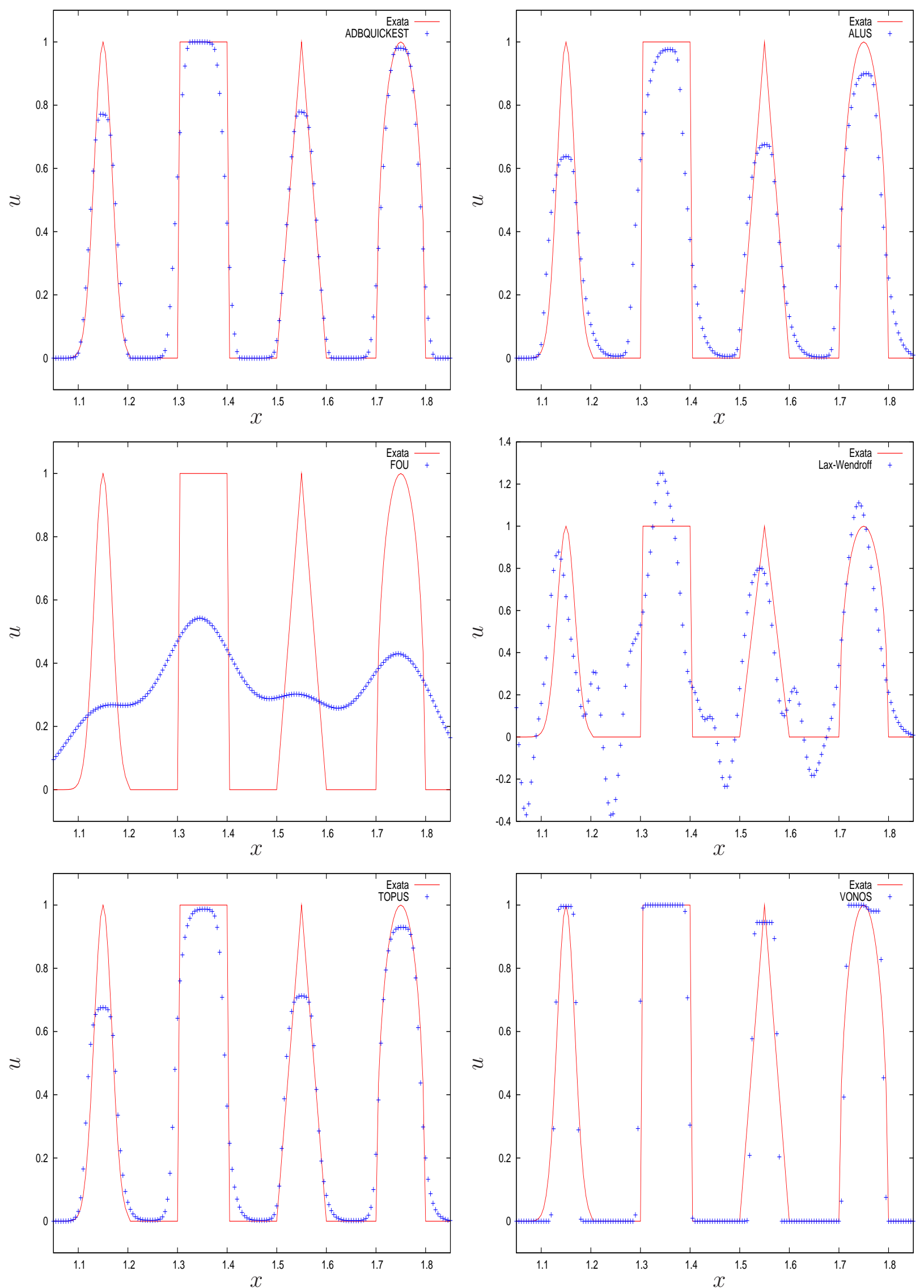

Figura 5.1: Simulação do caso 1 com número de Courant $\theta=0.05-$ Resultados numéricos para advecção de escalar usando os esquemas ADBQUICKEST, ALUS, FOU, Lax-Wendroff, TOPUS e VONOS 

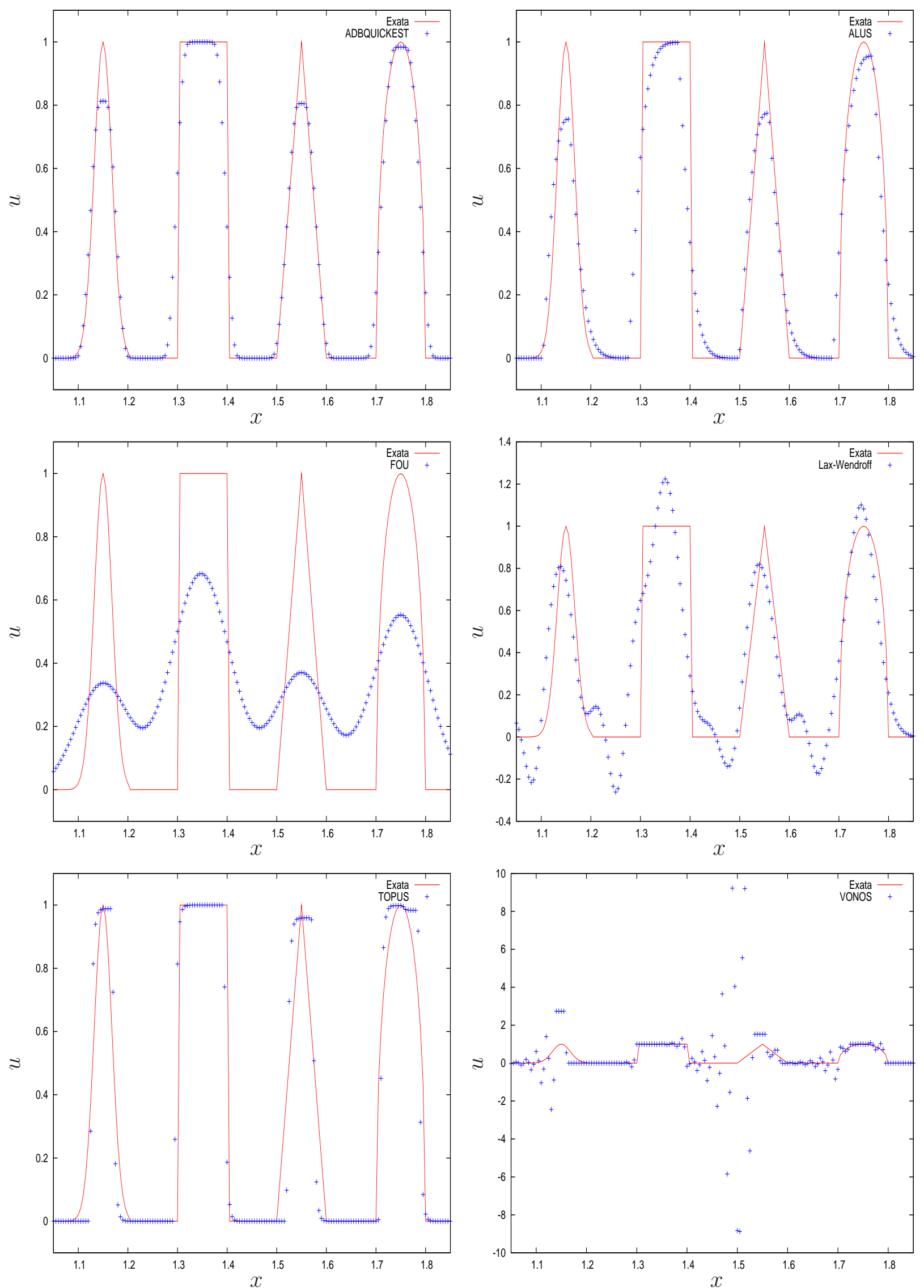

Figura 5.2: Simulação do caso 1 com número de Courant $\theta=0.5-$ Resultados numéricos para advecção de escalar usando os esquemas ADBQUICKEST, ALUS, FOU, Lax-Wendroff, TOPUS e VONOS. 

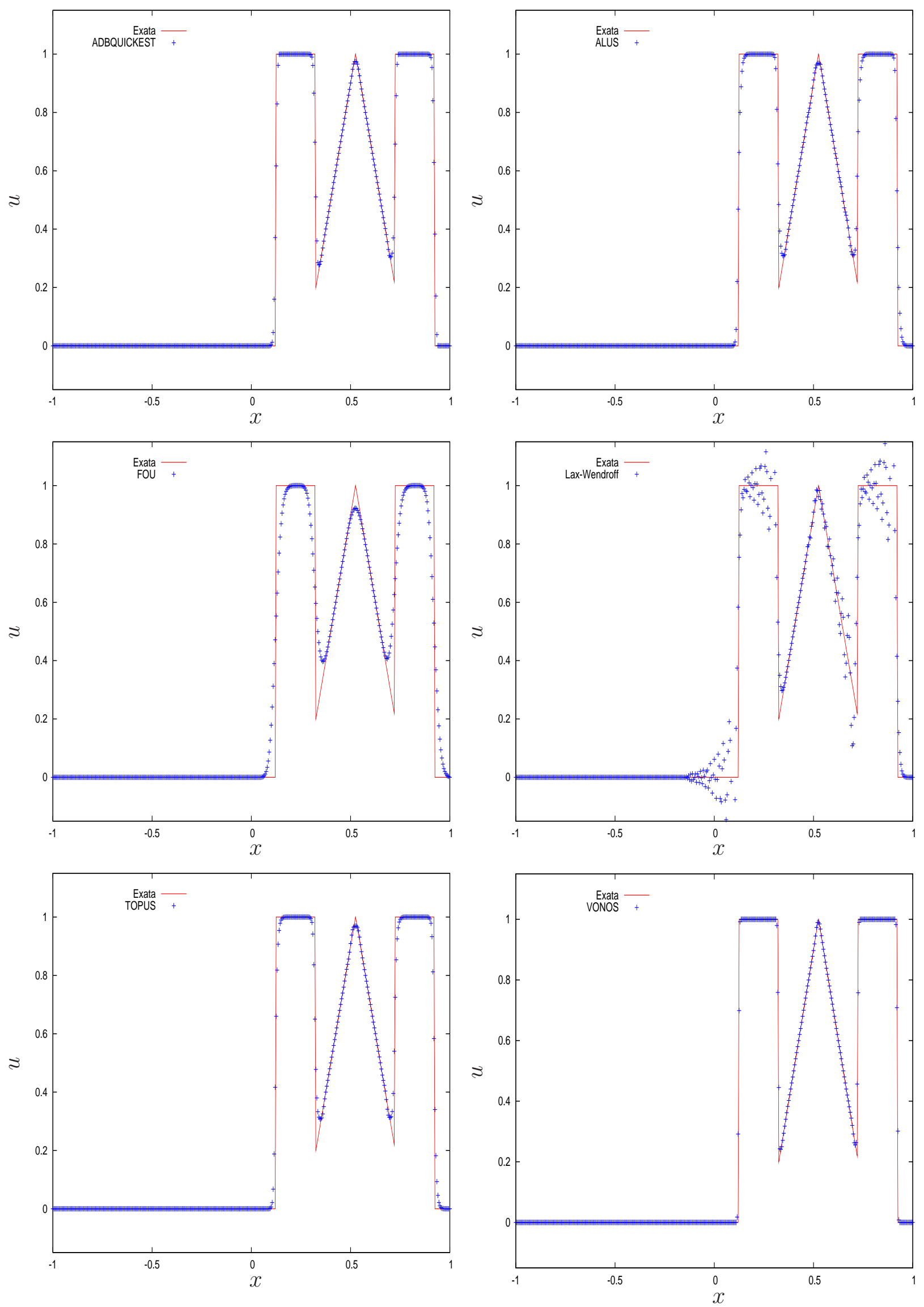

Figura 5.3: Simulação do caso 2 com número de Courant $\theta=0.05-$ Resultados numéricos para advecção de escalar usando os esquemas ADBQUICKEST, ALUS, FOU, Lax-Wendroff, TOPUS e VONOS. 

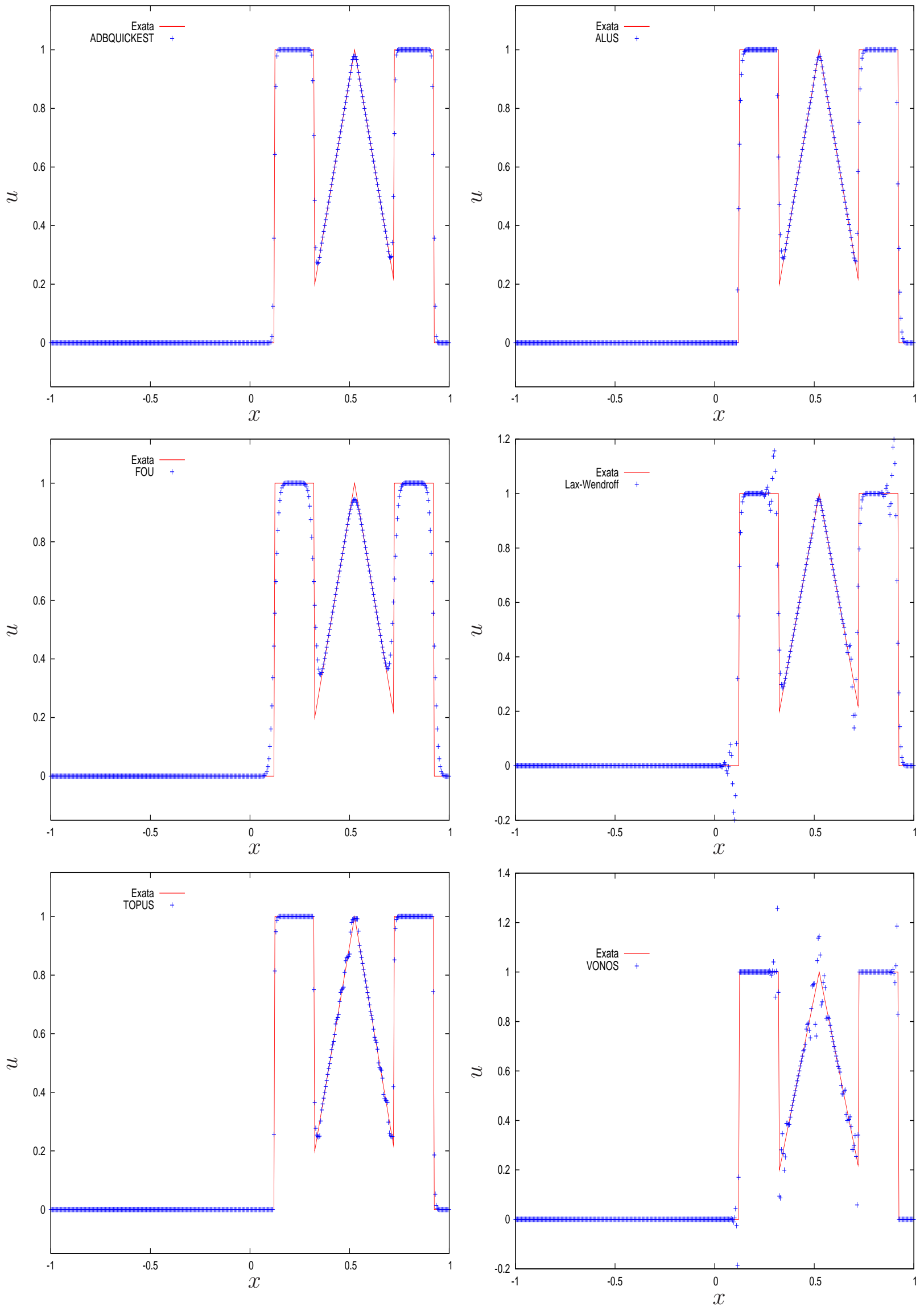

Figura 5.4: Simulação do caso 2 com número de Courant $\theta=0.5$ - Resultados numéricos para advecção de escalar usando os esquemas ADBQUICKEST, ALUS, FOU, Lax-Wendroff, TOPUS e VONOS. 

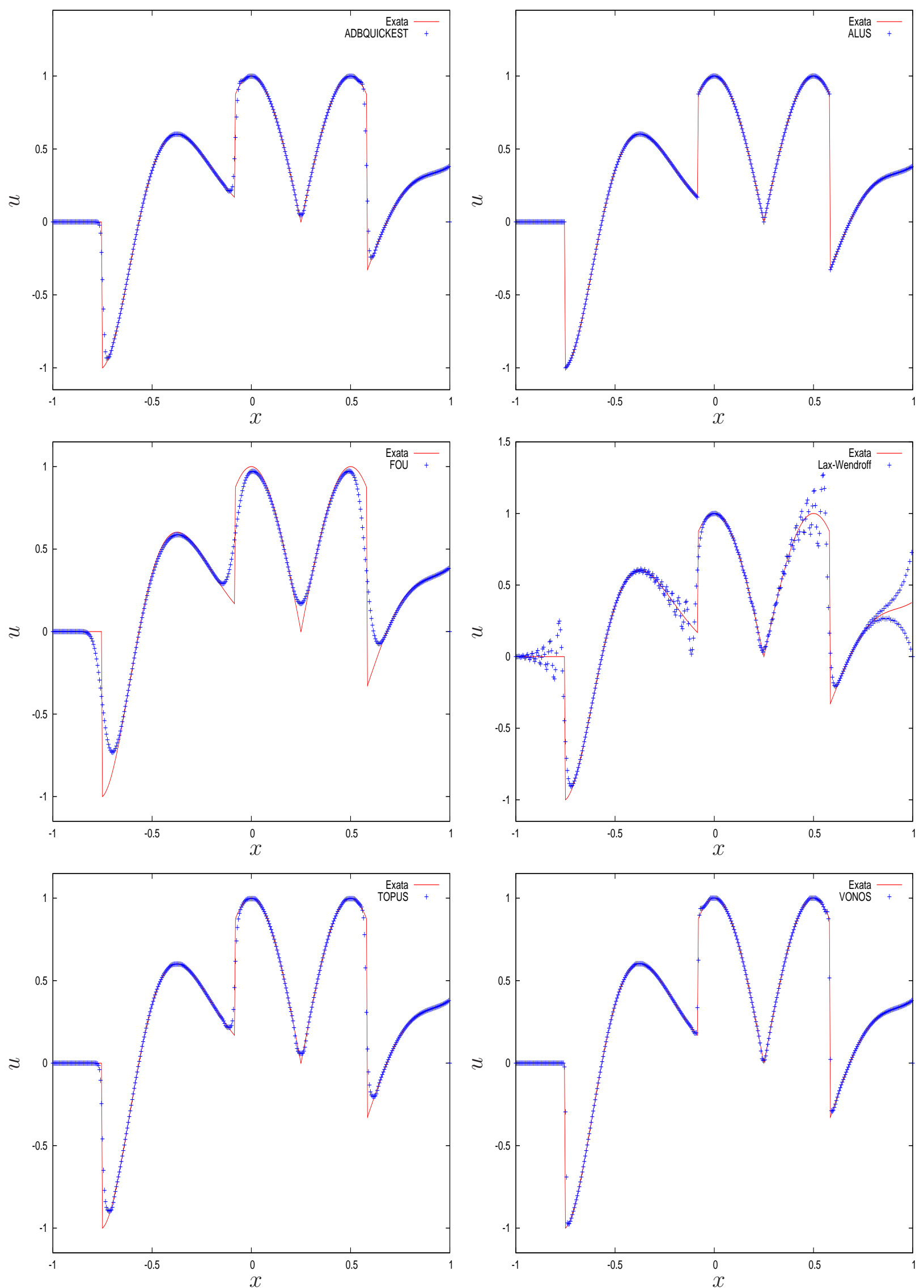

Figura 5.5: Simulação do caso 3 com número de Courant $\theta=0.05-$ Resultados numéricos para advecção de escalar usando os esquemas ADBQUICKEST, ALUS, FOU, Lax-Wendroff, TOPUS e VONOS. 

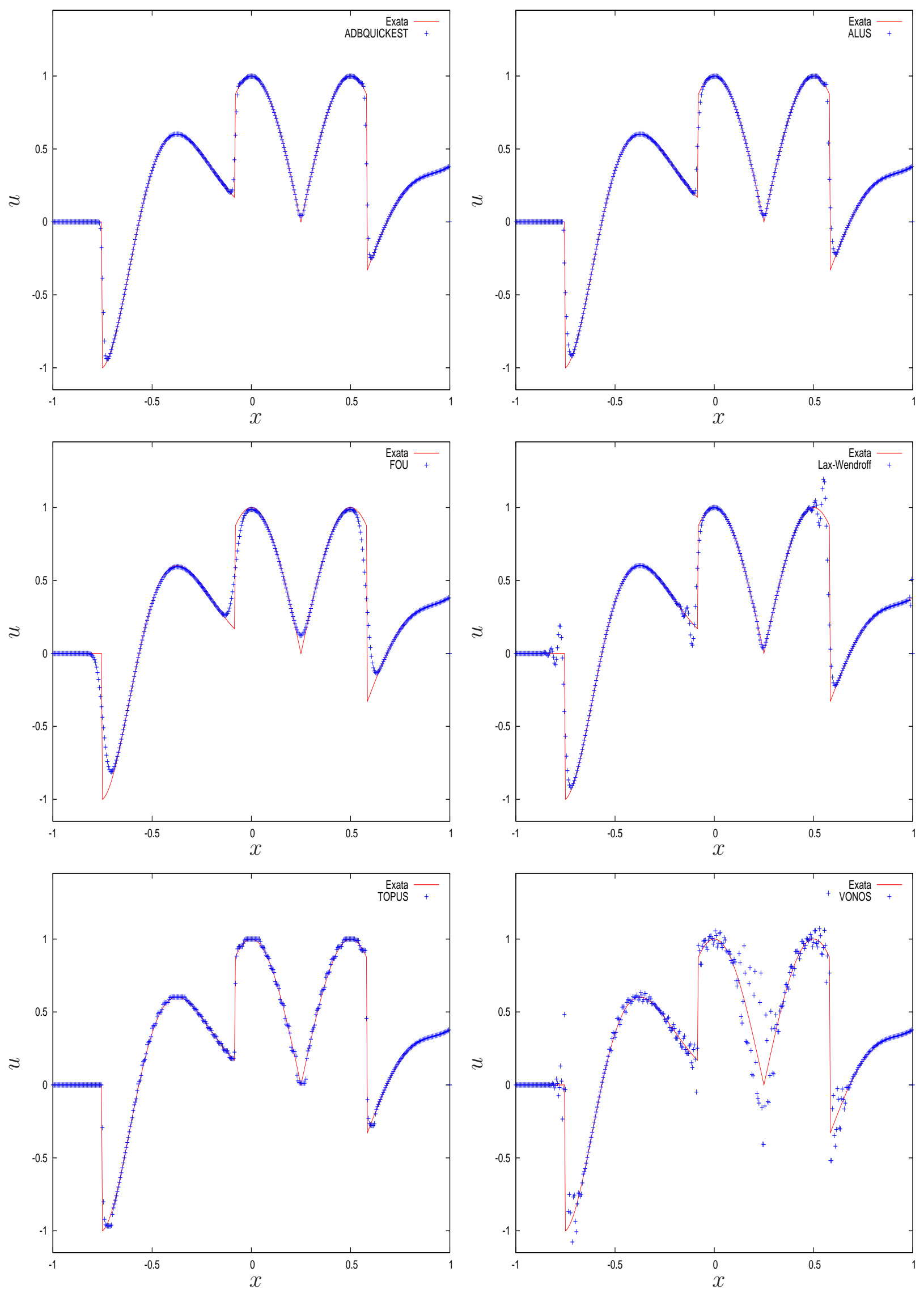

Figura 5.6: Simulação do caso 3 com número de Courant $\theta=0.5-$ Resultados numéricos para advecção de escalar usando os esquemas ADBQUICKEST, ALUS, FOU, Lax-Wendroff, TOPUS e VONOS. 


\subsection{Equação Linear de Convecção-Difusão}

Nesta seção são apresentadas soluções numéricas para a equação linear de convecção-difusão (2.4) com a condição (2.14) (problema "boundary layer"). Os resultados fornecidos pelo TOPUS foram parcialmente publicados em anais de evento (ver [114]). Para solução numérica dessa EDP no domínio $x \in[0,1]$, foram consideradas a condição inicial $u(x, 0)=0$ e a de contorno $u(0, t)=$ $u(1, t)=1$. A solução analítica deste problema é dada pela equação (2.15) [24]. As soluções exatas e numéricas a três viscosidades $(\nu=0.01,0.1$ e 1) são mostradas na Figura 5.7, onde $R e=1 / \nu$. Nessa figura, as soluções numéricas foram obtidas com os esquemas ADBQUICKEST, ALUS, SMART e TOPUS em três malhas diferentes ( $N=10,80$ e 640 células computacionais), com espaçamento temporal $\delta t=0.01 / N$ e tempo final de simulação $t=0.5$. Observa-se a partir dessa figura que os resultados numéricos com os esquemas ADBQUICKEST, ALUS, SMART e TOPUS estão, de maneira geral, em boa concordância com as soluções exatas e são bastante semelhantes entre si. É interessante destacar também que estes dados são consistentes com aqueles de Corre e Lerat [24] para este problema.

Na Tabela 5.1 são apresentadas estimativas, em várias normas, da taxa de convergência de cada um dos esquemas usando $\nu=0.02, \delta t=0.01 / N$ e tempo final $t=0.5$. Nota-se por essa tabela que as estimativas dessas taxas obtidas com os esquemas ALUS e TOPUS são superiores àquelas do ADBQUICKEST e SMART. Observa-se, ainda, por essa mesma tabela que o esquema TOPUS forneceu uma taxa maior entre as malhas de $N=80$ e $N=160$ células. Cabe mencionar ao leitor que o método numérico de diferenças finitas implementado com os esquemas "upwind" de alta resolução não consegue computar a solução numérica em malhas grosseiras com $R e=1 / \nu$ elevado.

\subsection{Problemas de Riemann para a Equação de Burgers}

A seguir dois casos envolvendo a obtenção da solução numérica de (2.4) com e sem viscosidade são descritos. Os resultados obtidos foram parcialmente publicados em anais de eventos e periódicos (ver [48, 103, 105, 109, 110, 111, 115]).

\section{Equação de Burgers com viscosidade}

Nesse caso, adota-se a equação (2.4), com a condição (2.8), e sujeita a três condições iniciais. Os esquemas ALUS e TOPUS foram utilizados nas simulações.

- Caso 1: neste caso são consideradas, para $x \in[0,1]$, as condições de contorno $u(0, t)=$ $\tanh (0.25 / \nu)$ e $u(1, t)=-\tanh (0.25 / \nu)$ e inicial $u(x, 0)=\tanh ((0.25-0.5 x) / \nu)$. A solução analítica desta EDP é definida em (2.10). Para acessar a convergência do processo numérico, as malhas de $N=100(\delta x=0.01), N=200(\delta x=0.005), N=400(\delta x=$ $0.0025)$ e $N=800(\delta x=0.00125)$ células computacionais foram adotadas. O espaçamento temporal foi $\delta t=0.0001$ e o tempo final de simulação $t=1.5$. Para a viscosidade utilizou-se $\nu=0.05$.

A Figura 5.8 mostra as soluções exata e numéricas obtidas usando os esquemas ALUS e TOPUS na malha de $N=100$ células. Dessa figura, percebe-se que a solução numérica é muito similar à exata. O resultado do estudo da convergência é apresentado na Tabela 5.2, onde nota-se que a segunda ordem foi atingida. Além disso, os resultados numéricos obtidos na simulação do esquema ALUS foram "ligeiramente" melhores que aqueles fornecidos pelo TOPUS. 

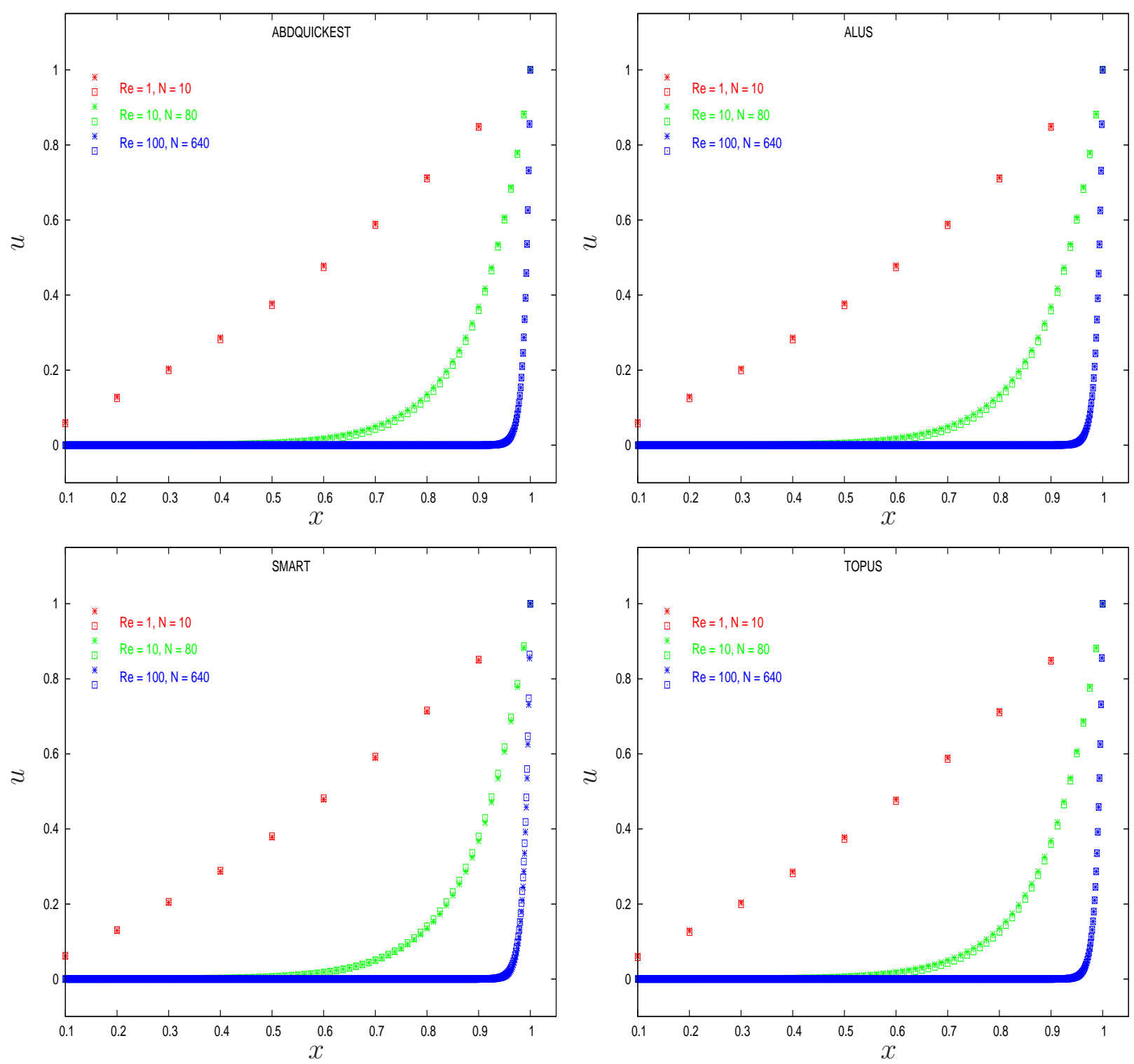

Figura 5.7: Soluções exatas ("quadrados") e resultados numéricos ("estrelas") para o problema da camada de limite obtidos com a utilização de esquemas "upwind" de alta resolução. 
Tabela 5.1: Estudo da convergência de esquemas convectivos de alta resolução, associado com um método de diferenças finitas explícito, aplicado a solução da equação da camada limite.

\begin{tabular}{cccccccc}
\hline & & Erro & Ordem & Erro & Ordem & Erro & Ordem \\
Esquema & $N$ & $\|E\|_{1}$ & $\|E\|_{1}$ & $\|E\|_{2}$ & $\|E\|_{2}$ & $\|E\|_{\infty}$ & $\|E\|_{\infty}$ \\
\hline ADBQUICKEST & 80 & $1.375 \times 10^{-2}$ & - & $1.012 \times 10^{-2}$ & - & $6.800 \times 10^{-3}$ & - \\
& 160 & $6.253 \times 10^{-3}$ & 1.137 & $4.476 \times 10^{-3}$ & 1.177 & $2.690 \times 10^{-3}$ & 1.338 \\
& 320 & $2.268 \times 10^{-3}$ & 1.463 & $1.621 \times 10^{-3}$ & 1.465 & $9.100 \times 10^{-4}$ & 1.564 \\
& 640 & $7.725 \times 10^{-4}$ & 1.554 & $5.596 \times 10^{-4}$ & 1.534 & $3.000 \times 10^{-4}$ & 1.601 \\
ALUS & & & & & & & \\
& 160 & $2.174 \times 10^{-2}$ & - & $1.619 \times 10^{-2}$ & - & $1.094 \times 10^{-2}$ & - \\
& 320 & $1.209 \times 10^{-4}$ & 2.233 & $8.420 \times 10^{-4}$ & 2.270 & $4.700 \times 10^{-4}$ & 2.382 \\
& 640 & $1.714 \times 10^{-4}$ & 2.828 & $1.081 \times 10^{-4}$ & 2.961 & $5.999 \times 10^{-5}$ & 2.972 \\
SMART & & & & & & & \\
& 80 & $2.081 \times 10^{-1}$ & - & $1.430 \times 10^{-1}$ & - & $9.215 \times 10^{-2}$ & - \\
& 160 & $1.271 \times 10^{-1}$ & 0.711 & $8.767 \times 10^{-2}$ & 0.706 & $5.065 \times 10^{-2}$ & 0.863 \\
& 320 & $7.035 \times 10^{-2}$ & 0.853 & $4.899 \times 10^{-2}$ & 0.840 & $2.697 \times 10^{-2}$ & 0.909 \\
& 640 & $3.701 \times 10^{-2}$ & 0.927 & $2.596 \times 10^{-2}$ & 0.916 & $1.391 \times 10^{-2}$ & 0.955 \\
& & & & & & & \\
TOPUS & 80 & $2.688 \times 10^{-2}$ & - & $1.966 \times 10^{-2}$ & - & $1.318 \times 10^{-2}$ & - \\
& 160 & $6.237 \times 10^{-3}$ & 2.108 & $4.466 \times 10^{-3}$ & 2.140 & $2.680 \times 10^{-3}$ & 2.298 \\
& 320 & $1.306 \times 10^{-3}$ & 2.256 & $9.398 \times 10^{-4}$ & 2.248 & $5.300 \times 10^{-4}$ & 2.338 \\
& 640 & $2.495 \times 10^{-4}$ & 2.388 & $1.891 \times 10^{-4}$ & 2.313 & $1.100 \times 10^{-4}$ & 2.295 \\
\hline
\end{tabular}
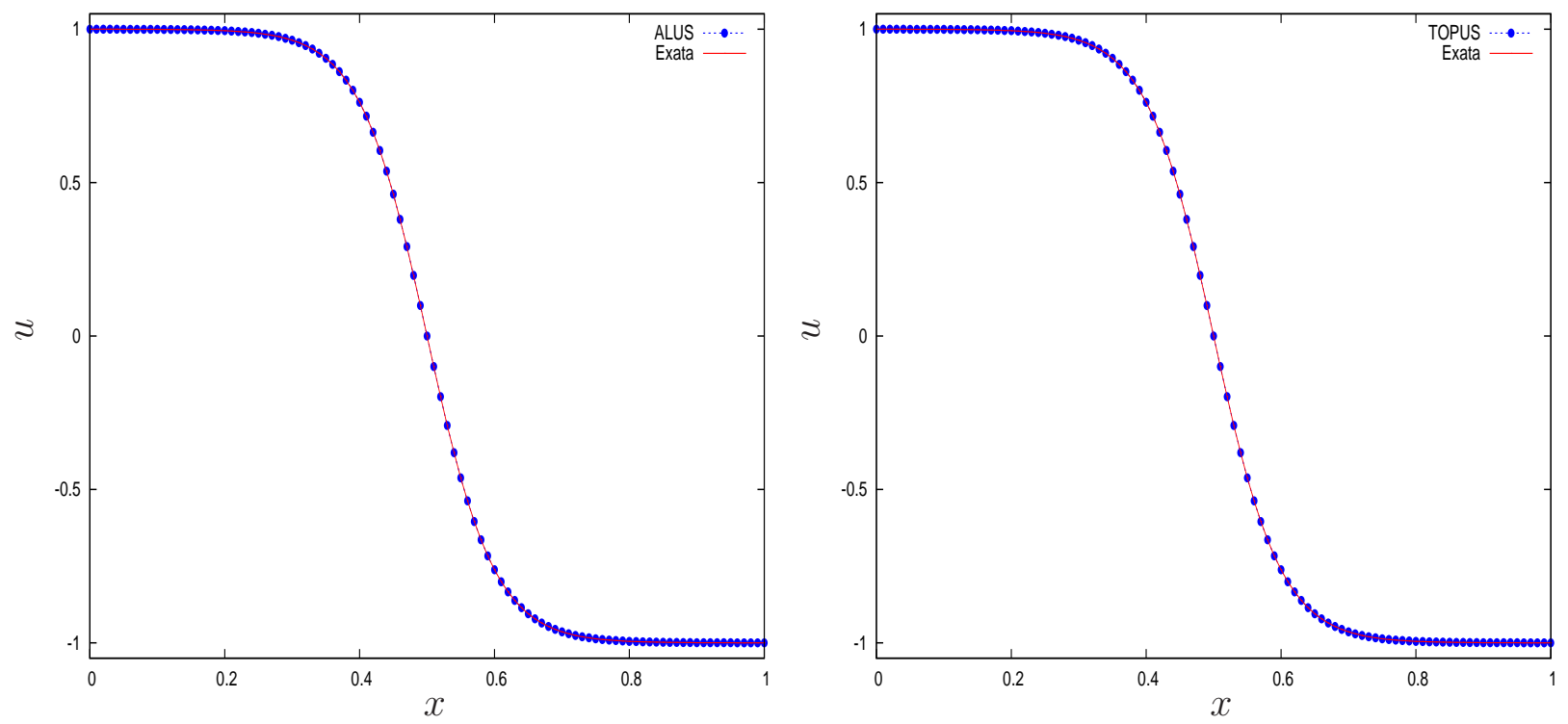

Figura 5.8: Caso 1: Comparação entre as soluções exata e numéricas da equação de Burgers. 
Tabela 5.2: Estudo da convergência dos esquemas ALUS e TOPUS aplicados à equação de Burgers com viscosidade $\nu=0.05$.

\begin{tabular}{|c|c|c|c|c|c|c|c|}
\hline Esquema & $N$ & $\begin{array}{c}\text { Erro } \\
\|E\|_{1}\end{array}$ & $\begin{array}{c}\text { Ordem } \\
\|E\|_{1}\end{array}$ & $\begin{array}{c}\text { Erro } \\
\|E\|_{2}\end{array}$ & $\begin{array}{c}\text { Ordem } \\
\|E\|_{2}\end{array}$ & $\begin{array}{c}\text { Erro } \\
\|E\|_{\infty}\end{array}$ & $\begin{array}{l}\text { Ordem } \\
\|E\|_{\infty}\end{array}$ \\
\hline \multirow[t]{4}{*}{ ALUS } & 100 & $9.916 \times 10^{-5}$ & - & $1.513 \times 10^{-4}$ & - & $3.100 \times 10^{-4}$ & - \\
\hline & 200 & $2.431 \times 10^{-5}$ & 2.028 & $3.764 \times 10^{-5}$ & 2.007 & $7.898 \times 10^{-5}$ & 1.973 \\
\hline & 400 & $5.985 \times 10^{-6}$ & 2.022 & $9.396 \times 10^{-6}$ & 2.002 & $2.003 \times 10^{-5}$ & 1.979 \\
\hline & 800 & $1.448 \times 10^{-6}$ & 2.047 & $2.330 \times 10^{-6}$ & 2.012 & $5.007 \times 10^{-6}$ & 2.000 \\
\hline \multirow[t]{4}{*}{ TOPUS } & 100 & $1.110 \times 10^{-4}$ & - & $1.677 \times 10^{-4}$ & - & $3.401 \times 10^{-4}$ & - \\
\hline & 200 & $3.052 \times 10^{-5}$ & 1.863 & $4.493 \times 10^{-5}$ & 1.900 & $8.900 \times 10^{-5}$ & 1.934 \\
\hline & 400 & $7.946 \times 10^{-6}$ & 1.941 & $1.164 \times 10^{-5}$ & 1.949 & $2.301 \times 10^{-5}$ & 1.952 \\
\hline & 800 & $2.012 \times 10^{-6}$ & 1.982 & $2.973 \times 10^{-6}$ & 1.969 & $6.021 \times 10^{-6}$ & 1.934 \\
\hline
\end{tabular}

- Caso 2: as condições iniciais e de contorno utilizadas, neste teste, são $u(x, 0)=\sin (2 \pi x)$ e $u(0, t)=u(1, t)=0$, respectivamente. Essas condições foram escolhidas com o objetivo de promover a formação do choque. Nas simulações foram adotados uma malha de 400 células computacionais $(\delta x=0.0025), \nu=0.001$, espaçamento temporal $\delta t=0.00125 \mathrm{e}$ tempo final de simulação $t=1$. A Figura 5.9 mostra as soluções numéricas em diferentes tempos. Nota-se a partir dessa figura, que houve a formação de uma descontinuidade na posição $x=0.5$, e que as soluções numéricas do ALUS e TOPUS capturaram este choque livre de oscilações. Os esquemas ALUS e TOPUS tiveram desempenho similar neste teste.
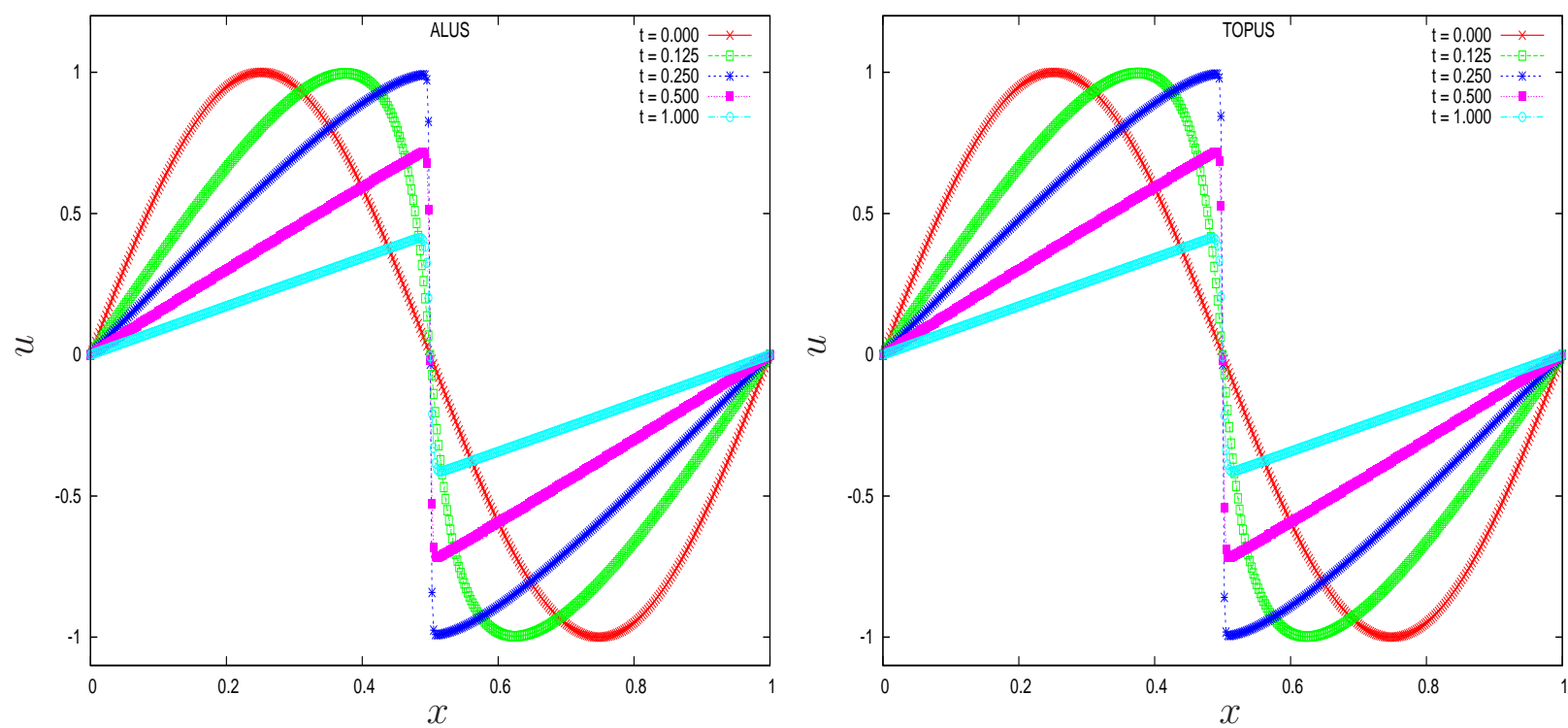

Figura 5.9: Caso 2: Soluções numéricas da equação de Burgers com viscosidade. 
- Caso 3: neste caso teste, as condições iniciais e de contorno adotadas foram, respectivamente, $u(x, 0)=1+\cos (x)$ e $u(0, t)=u(2 \pi, t)=1+\cos (t)$, em que $x \in[0,2 \pi]$. Na simulação desse teste foi empregada uma malha de 2500 células computacionais $(\delta x=0.0008 \pi)$, viscosidade $\nu=0.001$, passo temporal $\delta t=0.0001$ e tempo final de simulação $t=3.5$. Os perfis transientes obtidos com os esquemas ALUS e TOPUS estão mostrados na Figura 5.10. Da mesma forma como ocorreu anteriormente, os esquemas ALUS e TOPUS apresentaram soluções transientes livres de oscilações numéricas e boa resolução dos choques. Observa-se também que esses dados transientes são consistentes com aqueles apresentados por Fatkullin e Hesthaven [40].
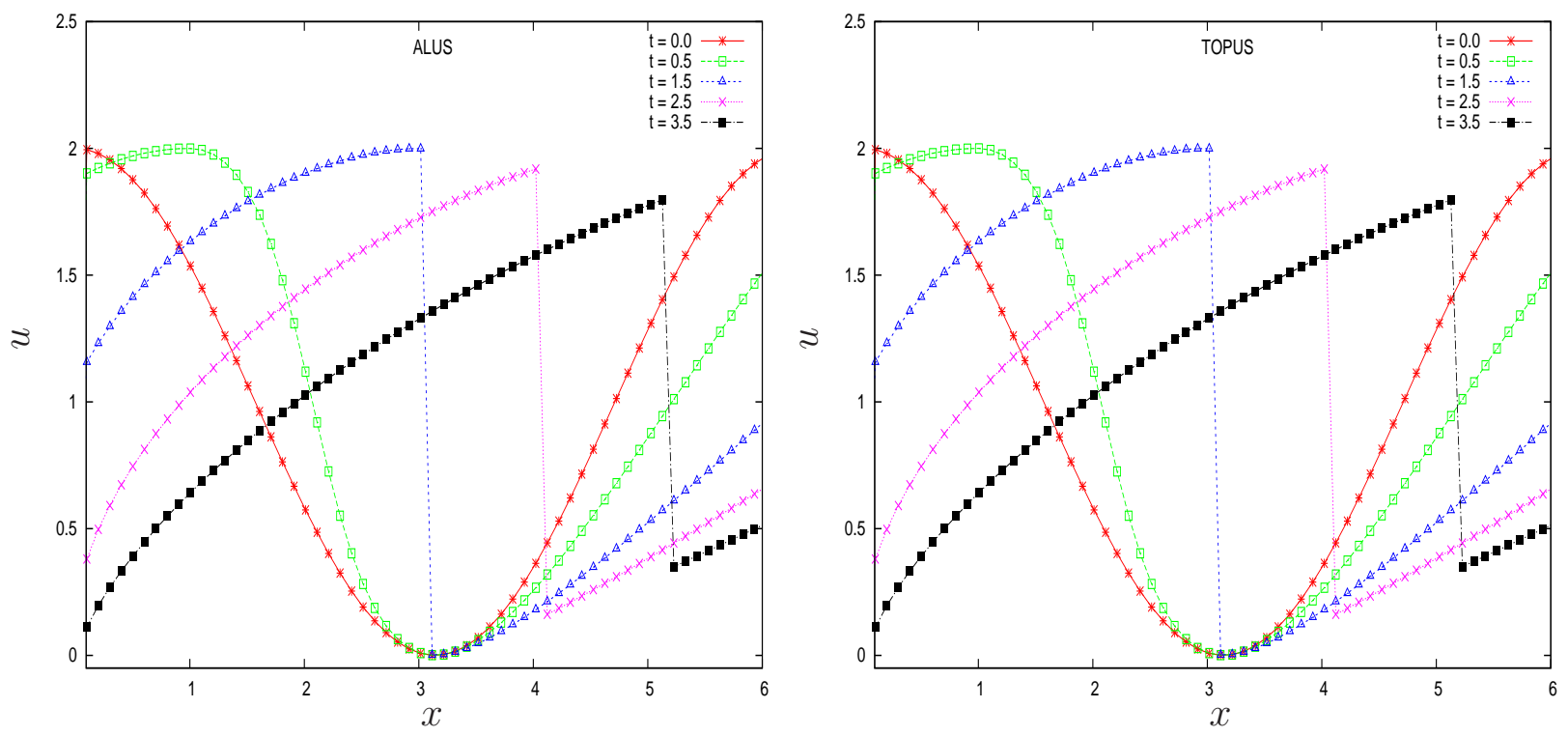

Figura 5.10: Caso 3: Soluções numéricas da equação de Burgers com viscosidade.

\section{Equação de Burgers sem viscosidade}

A equação de Burgers sem viscosidade é dada pela equação (2.4) adotando as condições (2.9). Seguem dois casos aqui investigados, visando a obtenção da solução numérica de problemas mais complexos.

- Caso 1 (Onda de choque) [1]: neste caso, a condição inicial (equação (2.11)) adotada é $u_{L}=0.8$ e $u_{R}=0.2$ para $x \in[-1,1]$. Para a simulação foram utilizados uma malha de 200 células computacionais $(\delta x=0.01)$, marcha no tempo $\delta t=0.009$ e tempo final de simulação $t=0.5$. Nas Figuras 5.11a e 5.12a são apresentados os resultados numéricos com ALUS e TOPUS mais a solução exata dada em (2.12) [1]. Vê-se por essa figura que houve boa concordância entre as soluções numérica e exata. Nota-se também, por essa mesma figura, que ocorreu vestígios de difusão numérica próximo ao ponto $x=0.225$. Os erros $\|E\|_{2}$ (ver equação (2.69)) - cometidos pelos esquemas ALUS e TOPUS foram $4.3 \times 10^{-2} \mathrm{e}$ $4.4 \times 10^{-2}$, respectivamente. As figuras $5.11 \mathrm{~b}$ e $5.12 \mathrm{~b}$ mostram os perfis transientes obtidos. Mais uma vez, é possível notar que essas soluções numéricas estão livres de oscilações. Vale destacar, ainda, que os resultados numéricos aqui apresentados são superiores aqueles obtidos por Ahmed [1] usando os esquemas FOU, Lax-Wendroff e Warming-Beam [153]. 
(a)

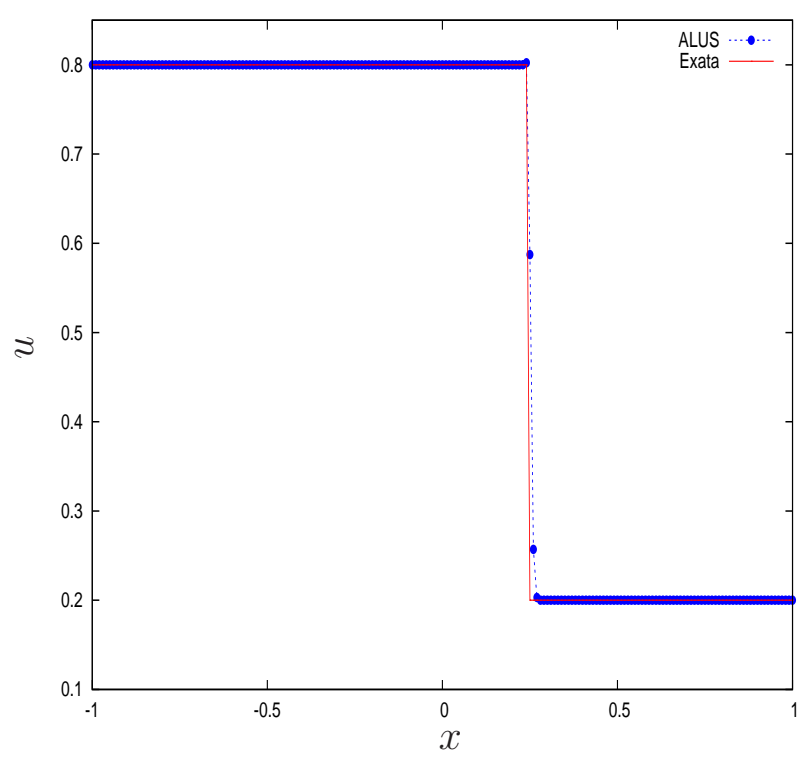

(b)

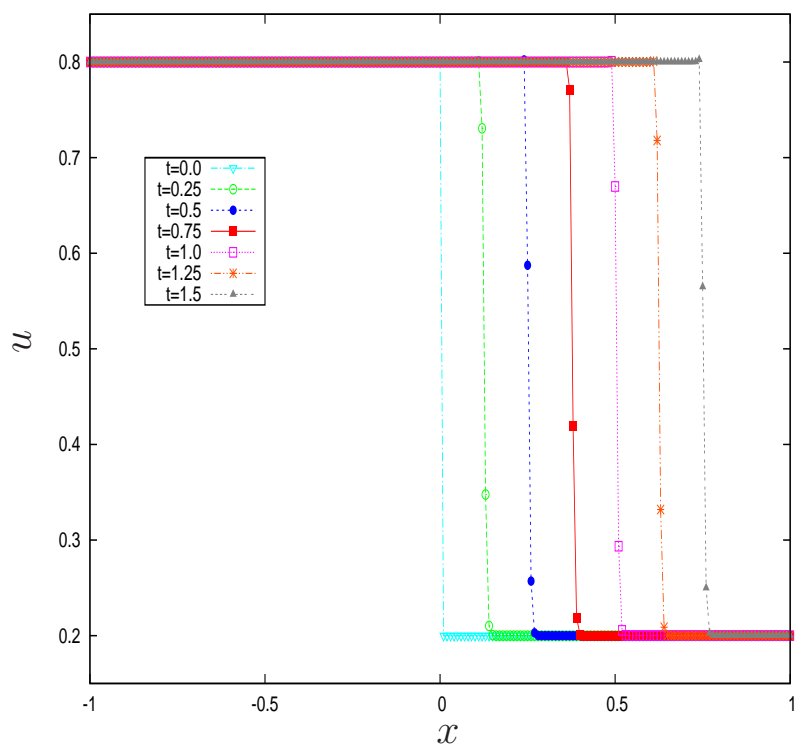

Figura 5.11: Simulação numérica de uma onda de choque usando o ALUS: (a) comparação entre as soluções numérica e exata; (b) perfil transiente da solução numérica.

(a)

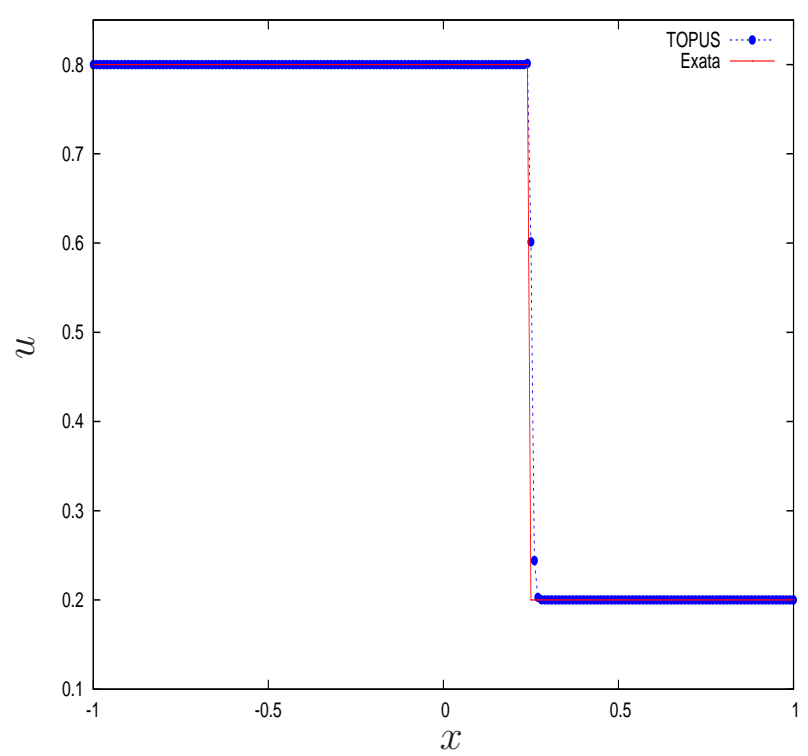

(b)

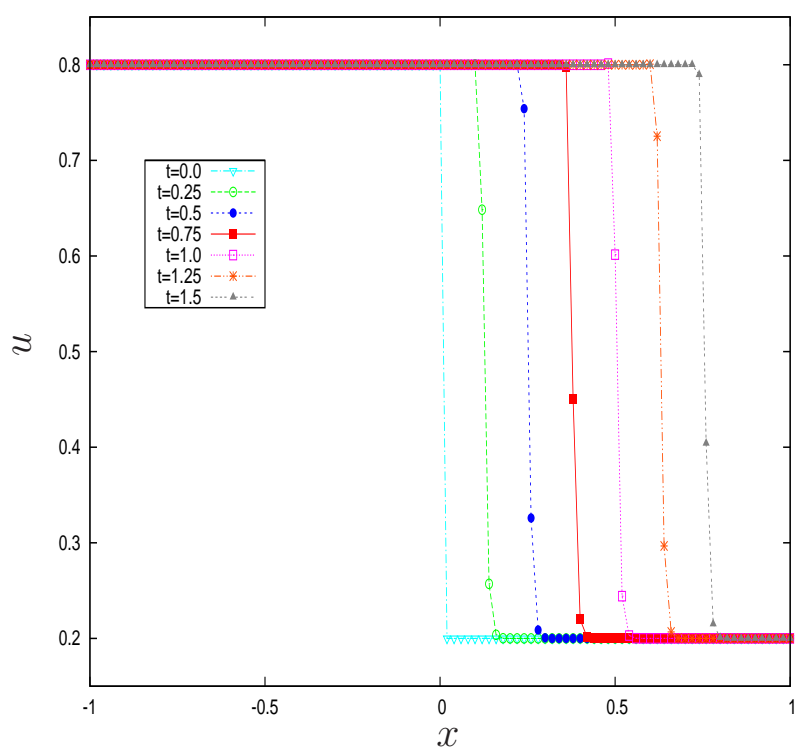

Figura 5.12: Simulação numérica de uma onda de choque usando o TOPUS: (a) comparação entre as soluções numérica e exata; (b) perfil transiente da solução numérica. 
- Caso 2 (Onda de rarefação) [1]: neste teste, a condição inicial (2.11) é $u_{L}=0.0$ e $u_{R}=0.5$ para $x \in[-1.5,1]$. Nessa simulação, o tempo final de simulação é $t=2.0$, o espaçamento temporal $\delta t=0.01625$ e a malha empregada possui 200 células computacionais $(\delta x=$ 0.0125). O problema foi simulado usando-se os esquemas convectivos ALUS, Superbee [118], TOPUS e VONOS. Na Figura 5.13 é apresentada a comparação entre as soluções numéricas e a exata dada por (2.13) [1]. Pode-se notar por essa figura que o resultado obtido com o esquema TOPUS foi melhor que aqueles dos outros esquemas. Observou-se também que o esquema ALUS apresentou dissipação numérica próximo ao ponto $x=0$.
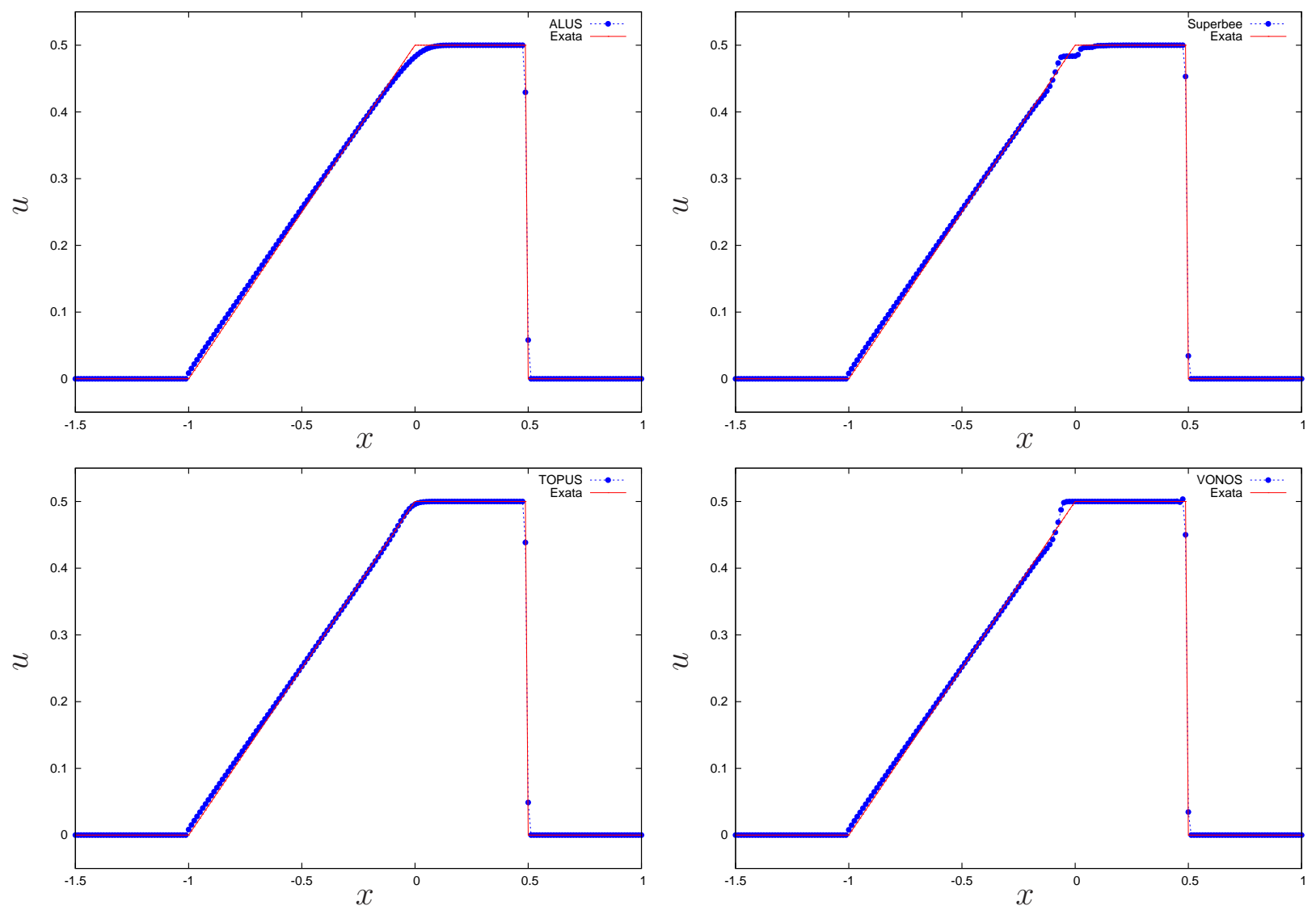

Figura 5.13: Simulação numérica de uma onda de rarefação usando os esquemas convectivos ALUS, Superbee, TOPUS e VONOS. 


\subsection{Equação de Buckley-Leverett}

Nesta seção são apresentados resultados numéricos obtidos para a equação de Buckley-Leverett [7] definida em (2.4) com (2.7). Para resolver esta complicada equação, foram utilizados os esquemas ALUS, FOU e TOPUS. Como Ahmed [1], dois casos de teste foram simulados:

- Caso 1: neste teste, a condição inicial (2.5) é dada por

$$
u(x, 0)= \begin{cases}0.5, & x=0, \\ 0, & x \in(0,1] .\end{cases}
$$

A malha empregada na simulação possui 30 células $(\delta x=0.033)$. O tempo final de simulação e o espaçamento temporal foram $t=0.162$ e $\delta t=0.009$, respectivamente. O resultado numérico está ilustrado na Figura 5.14a. Nota-se que o FOU apresentou comportamento difusivo. Também, observa-se que os esquemas ALUS e TOPUS não produziram oscilações numéricas na região da descontinuidade $(x=0.26)$.

- Caso 2: a condição inicial (2.5) abordada neste caso é expressa por

$$
u(x, 0)= \begin{cases}0.75, & x=0, \\ 0, & x \in(0,3] .\end{cases}
$$

A malha considerada nesta simulação tem 50 células $(\delta x=0.06)$. O tempo final de simulação e o espaçamento temporal foram $t=0.9$ e $\delta t=0.009$, respectivamente. O resultado numérico está ilustrado na Figura 5.14b. Mais uma vez, na região do choque, os esquemas ALUS e TOPUS produziram soluções numéricas livres de oscilações.

(a)

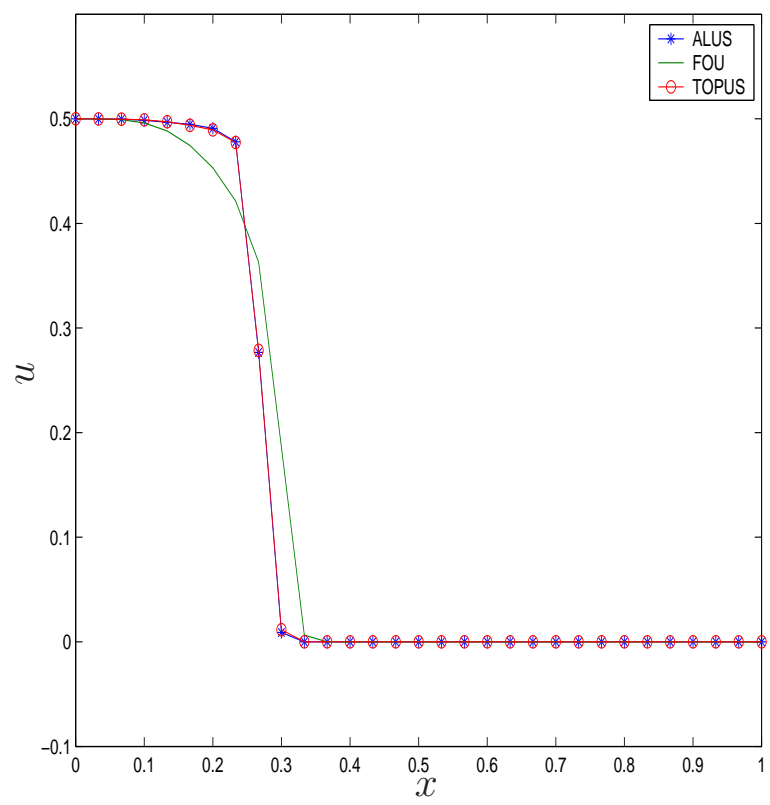

(b)

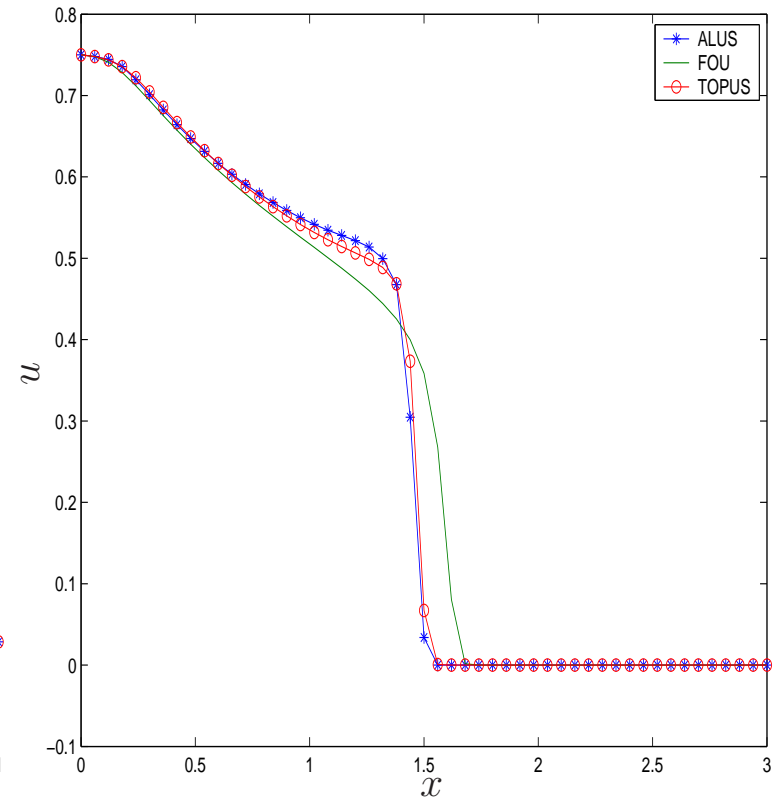

Figura 5.14: Resultados numéricos da equação de Buckley-Leverett: (a) caso 1; (b) caso 2.

É importante destacar que os resultados numéricos fornecidos nas simulações do ALUS e TOPUS são superiores aqueles obtidos por Ahmed [1] utilizando os esquemas Lax-Wendroff, Lax-Friedrichs e Warming-Beam. 


\subsection{Equação de Águas Rasas}

O problema conhecido por "dam-break" (ver [82]), modelado pelo sistema hiperbólico 1D (2.16), aparece freqüentemente na literatura com o objetivo de testar novos esquemas numéricos. Ele também é adotado neste trabalho para testar os esquemas convectivos ADBQUICKEST, ALUS, Superbee e TOPUS. Para tanto, a condição inicial é dada por

$$
\begin{aligned}
& h(x, 0)= \begin{cases}3, & x \in[-5,0], \\
1, & x \in(0,5],\end{cases} \\
& u(x, 0)=0 .
\end{aligned}
$$

Para a resolução do problema, foi utilizado o pacote computacional CLAWPACK de LeVeque [82] equipado com os limitadores de fluxo dos esquemas ADBQUICKEST, ALUS, Superbee e TOPUS. Este robusto código computa a solução numérica de equações diferenciais hiperbólicas no contexto de volumes finitos usando uma abordagem de propagação de onda (ver detalhes em [82]) . Neste teste foram empregados uma malha de 100 células computacionais $(\delta x=0.1) \mathrm{e}$ tempo final de simulação $t=2$. As Figuras 5.15 e 5.16 mostram as soluções numéricas e de referência (usando o método Godunov [82] e uma malha de 1000 células), para a profundidade $h$ e vazão $h u$, respectivamente. Por essas figuras, observa-se que os esquemas Superbee e TOPUS produziram soluções numéricas de boa qualidade e em concordância com a de referência.

CLAWPACK is a software package designed to compute numerical solutions to hyperbolic partial differential equations using a wave propagation approach. 

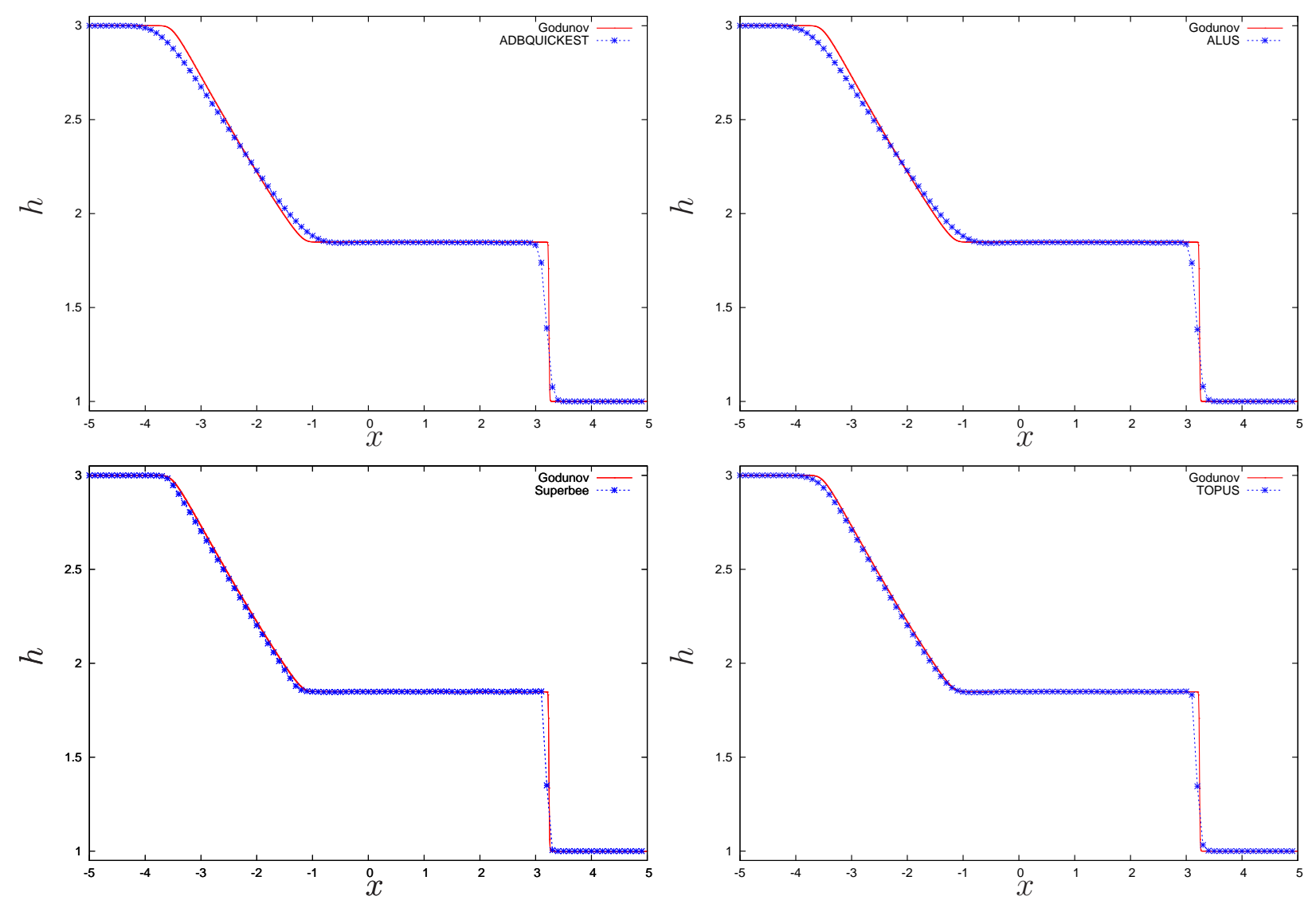

Figura 5.15: Soluções numéricas e de referência para a profundidade $h$ no problema "dam-break". 

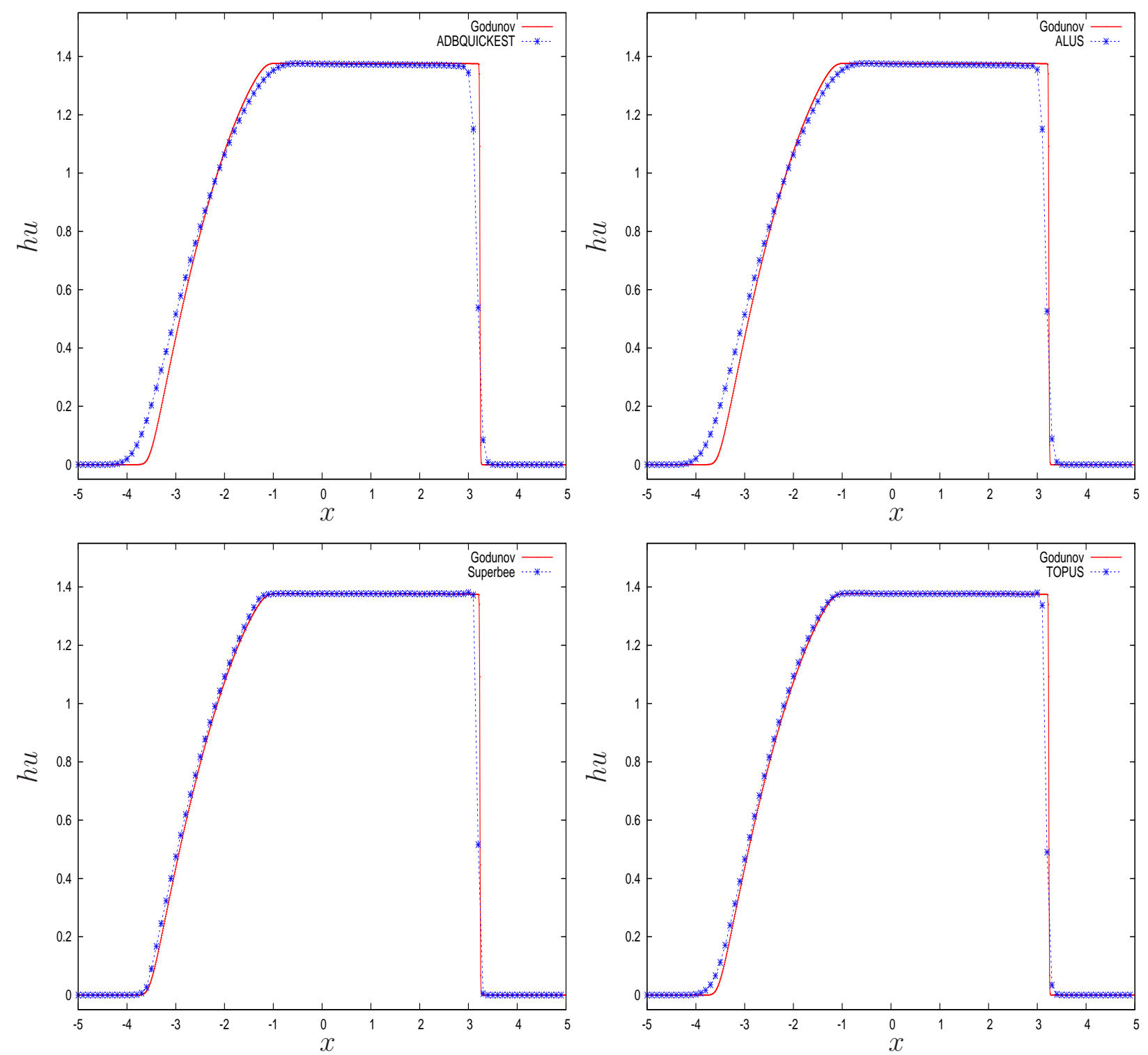

Figura 5.16: Soluções numéricas e de referência para a vazão hu no problema "dam-break". 


\subsection{Problemas de Riemann para as Equações de Euler}

Este é um problema interessante e que aparece também com freqüência na literatura para testar esquemas numéricos. A seguir, três problemas de Riemann são simulados com o uso dos esquemas ADBQUICKEST, ALUS, ARORA [10], Lax-Wendroff, MINMOD [119], SMART, Superbee e TOPUS. Os resultados expostos nesta seção foram parcialmente publicados em anais de eventos e periódicos [48, 75, 106, 109, 110, 114, 115].

\subsubsection{Tubo de Choque de Sod}

Neste problema, proposto por Sod [130], é considerada a equação (2.18), $x \in[0,1]$, sujeita às condições iniciais

$$
[\rho, u, p]^{T}= \begin{cases}{[1,0,1]^{T},} & x \in[0,0.5), \\ {[0.125,0,0.1]^{T},} & x \in[0.5,1] .\end{cases}
$$

Nesse problema, foram considerados o tempo final de simulação (instante de ocorrência do choque) $t=0.2$ e o espaçamento temporal $\delta t=0.6 \delta x$. Duas malhas com $N=50(\delta x=0.02)$ e $N=200(\delta x=0.005)$ células computacionais foram adotados nas simulações. A Figura 5.17 mostra as soluções numéricas, usando os esquemas ADBQUICKEST, ALUS, Lax-Wendroff, SMART, Superbee e TOPUS, e de referência (FOU na malha de 2000 células - $\delta x=0.0005$ ). Nota-se por meio dessa figura que o TOPUS resolveu bem o choque, quando comparado com os demais esquemas. Cabe mencionar também que a convergência das soluções numéricas para a de referência foi melhor para o esquema TOPUS.

\subsubsection{Tubo de Choque de Shu-Osher}

Neste tubo de choque, proposto por Shu-Osher [124], é considerada a equação (2.18), $x \in$ $[-1,3]$, suplementada com a condição inicial

$$
[\rho, u, p]^{T}= \begin{cases}{[3.86,2.63,10.33]^{T},} & x \in[-1,-0.8), \\ {[1+0.2 \sin (5 x), 0,1]^{T},} & x \in[-0.8,3] .\end{cases}
$$

Para a simulação deste problema, o tempo final de processamento foi $t=1$ (instante de ocorrência do choque) e espaçamento temporal de $\delta t=0.6 \delta x$. Duas malhas com $N=200(\delta x=0.02) \mathrm{e}$ $N=300(\delta x=0.0133)$ células computacionais foram adotadas. A solução de referência para esse problema foi obtida da mesma forma como feito no tubo de choque de Sod. A Figura 5.18 apresenta as soluções numéricas obtidas usando os esquemas ADBQUICKEST, ALUS, Lax-Wendroff, SMART, Superbee e TOPUS e a de referência. Vê-se por essa figura, que a simulação usando-se o TOPUS forneceu resultados em boa concordância com a solução de referência. Ainda mais, o esquema ALUS teve comportamento similar ao do ADBQUICKEST. 

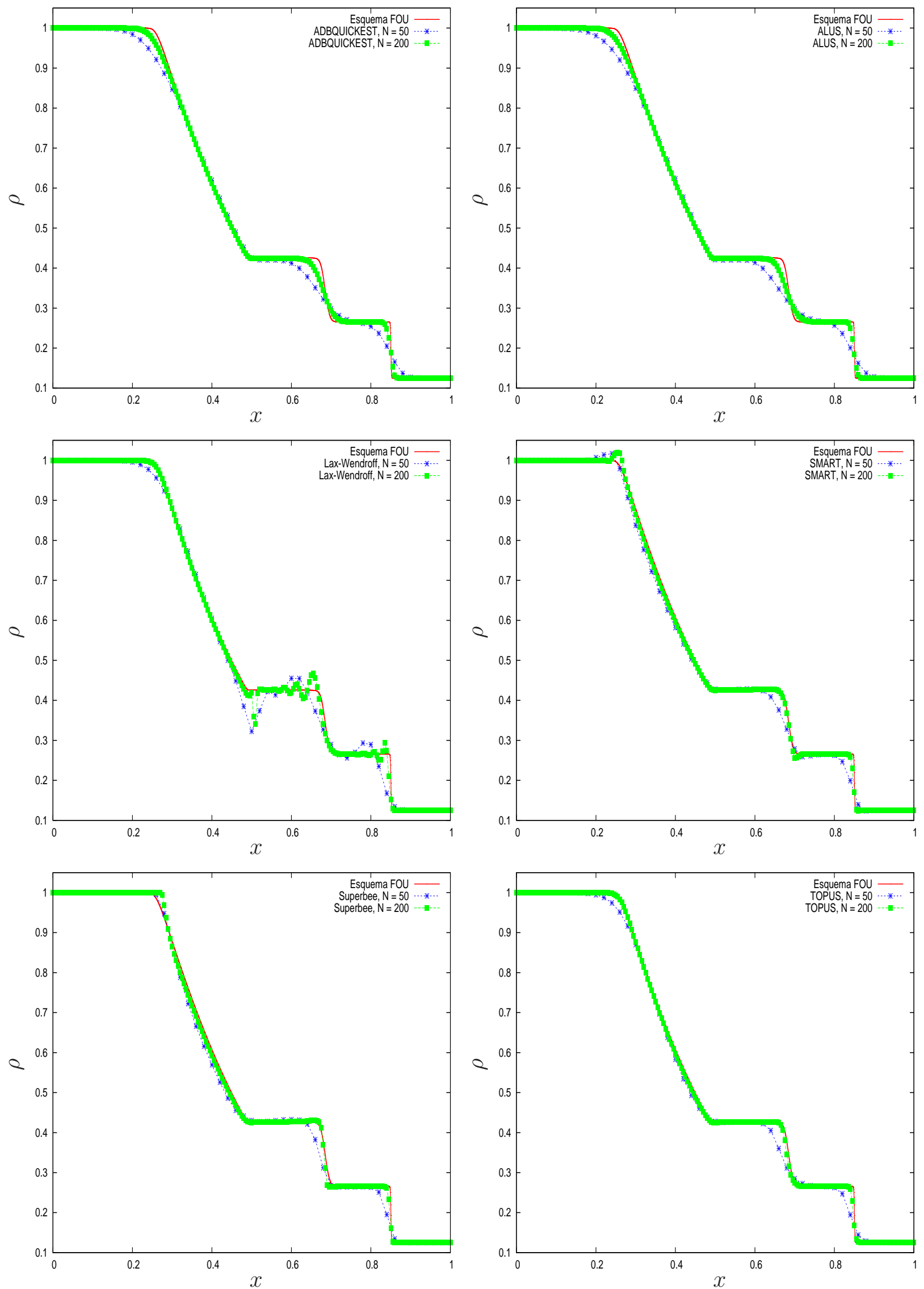

Figura 5.17: Soluções numéricas do tubo de choque de Sod obtidas com os esquemas ADBQUICKEST, ALUS, Lax-Wendroff, SMART, Superbee e TOPUS. 

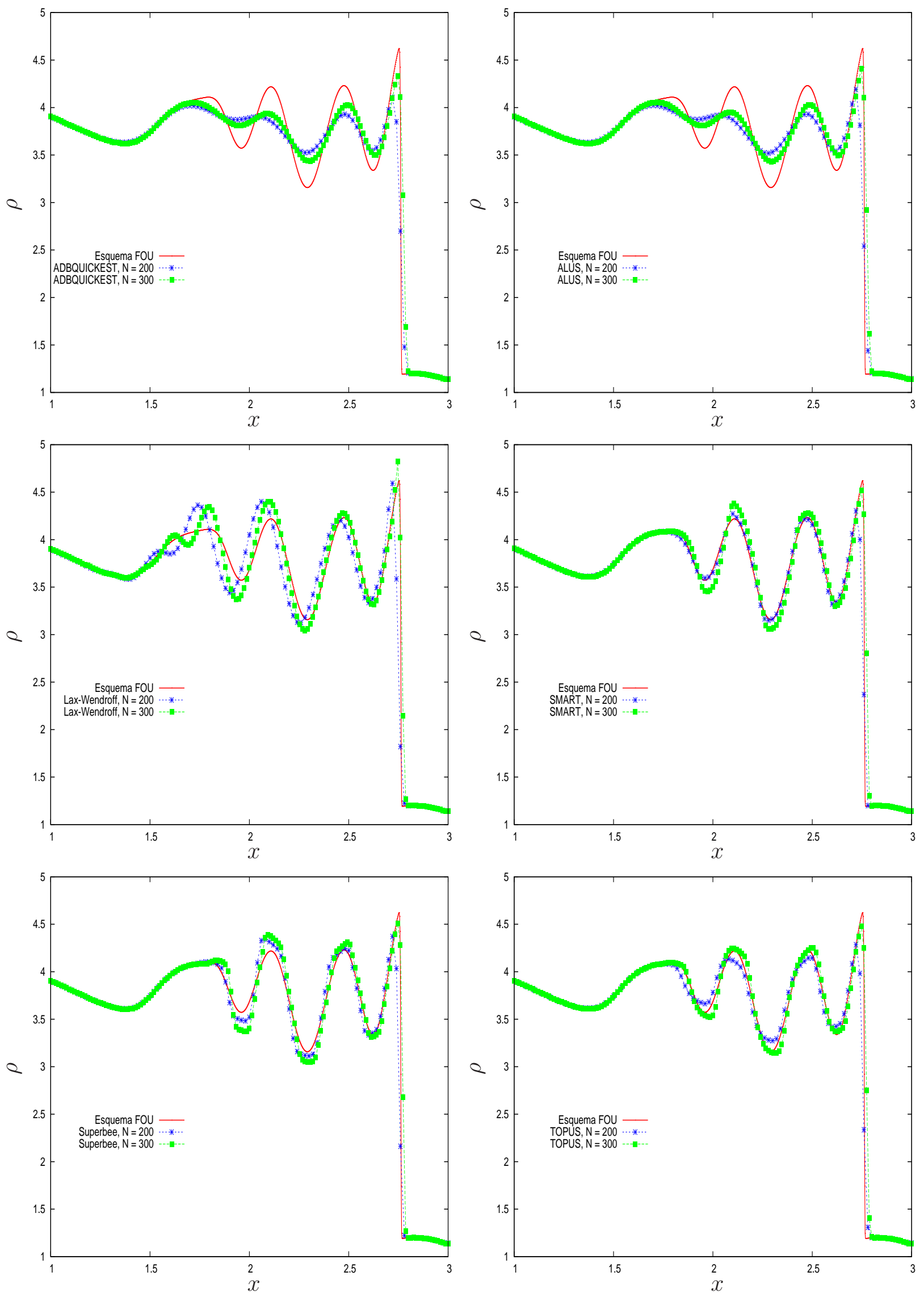

Figura 5.18: Soluções numéricas do tubo de choque de Shu-Osher obtidas com os esquemas ADBQUICKEST, ALUS, Lax-Wendroff, SMART, Superbee e TOPUS. 


\subsubsection{Tubo de Choque de Shu-Osher Modificado}

Este problema de Riemann, proposto por Titarev e Toro [144], é uma variação do tubo de choque de Shu-Osher [124]. Ele constitui um excelente teste para esquemas "upwind" de alta resolução. Considera-se a equação (2.18), $x \in[-5,5]$, com condições iniciais dadas por

$$
[\rho, u, p]^{T}= \begin{cases}{[1.515695,0.523346,1.805]^{T},} & x \in[-5,4.5), \\ {[1+0.1 \sin (20 \pi x), 0,1]^{T},} & x \in[4.5,5] .\end{cases}
$$

Para a simulação deste problema, foram empregados os seguintes dados: tempo final de processamento $t=5$ (instante de ocorrência do choque); espaçamento temporal $\delta t=0.0024$; e malha computacional de $N=2500$ células computacionais $(\delta x=0.004)$. A solução de referência foi considerada usando o esquema FOU numa malha de 20000 células $(\delta x=0.0005)$. A Figura 5.19 apresenta as soluções numéricas obtidas usando os esquemas ADBQUICKEST, ALUS, ARORA, MINMOD, Superbee e TOPUS e de referência. Novamente, com base nessa figura, fica clara a superioridade do esquema TOPUS.

\subsubsection{Tubos de Choque de Toro}

Neste tubo de choque, proposto por Toro [147], é considerada a equação (2.18), $x \in[0,1]$, sujeita a duas condições iniciais.

- Caso 1: neste caso, a condição inicial é expressa por

$$
[\rho, u, p]^{T}= \begin{cases}{[1,0,1000]^{T},} & x \in[0,0.5), \\ {[1,0,0.01]^{T},} & x \in[0.5,1] .\end{cases}
$$

Para a simulação desse caso, o tempo final de processamento $t=0.012$ (instante do choque), o espaçamento temporal $\delta t=0.6 \delta x$, e duas malhas de $N=200(\delta x=0.05)$ e $N=400$ $(\delta x=0.0025)$ células foram utilizados. A solução de referência para este problema foi obtida com a simulação do esquema FOU numa malha de 4000 células ( $\delta x=0.00025$ ). A Figura 5.20 mostra as soluções de referência e as numéricas obtidas nas simulações dos esquemas ADBQUICKEST, ALUS, ARORA, MINMOD, SMART e TOPUS. Dessa figura, percebe-se que as soluções obtidas com MINMOD e TOPUS são livres de difusão numérica. Também nota-se que essas soluções são bastante consistentes com a de referência.

- Caso 2: neste caso, a condição inicial é dada por

$$
[\rho, u, p]^{T}= \begin{cases}{[5.99924,19.5975,460.894]^{T},} & x \in[0,0.4), \\ {[5.99242,-6.19633,46.095]^{T},} & x \in[0.4,1] .\end{cases}
$$

Para a simulação desse caso, o tempo final de processamento $t=0.035$ (instante do choque) foi utilizado. $\mathrm{O}$ espaçamento temporal, as malhas empregadas e a solução de referência foram iguais a do caso descrito anteriormente. A Figura 5.21 apresenta as soluções de referência e as numéricas obtidas nas simulações usando os esquemas ADBQUICKEST, ALUS, ARORA, SMART, Superbee e TOPUS. Dessa figura, nota-se que as simulações dos esquemas Superbee e TOPUS produziram resultados mais próximos da solução de referência, com a convergência do método usando o TOPUS sendo mais satisfatória do que com o Superbee. 

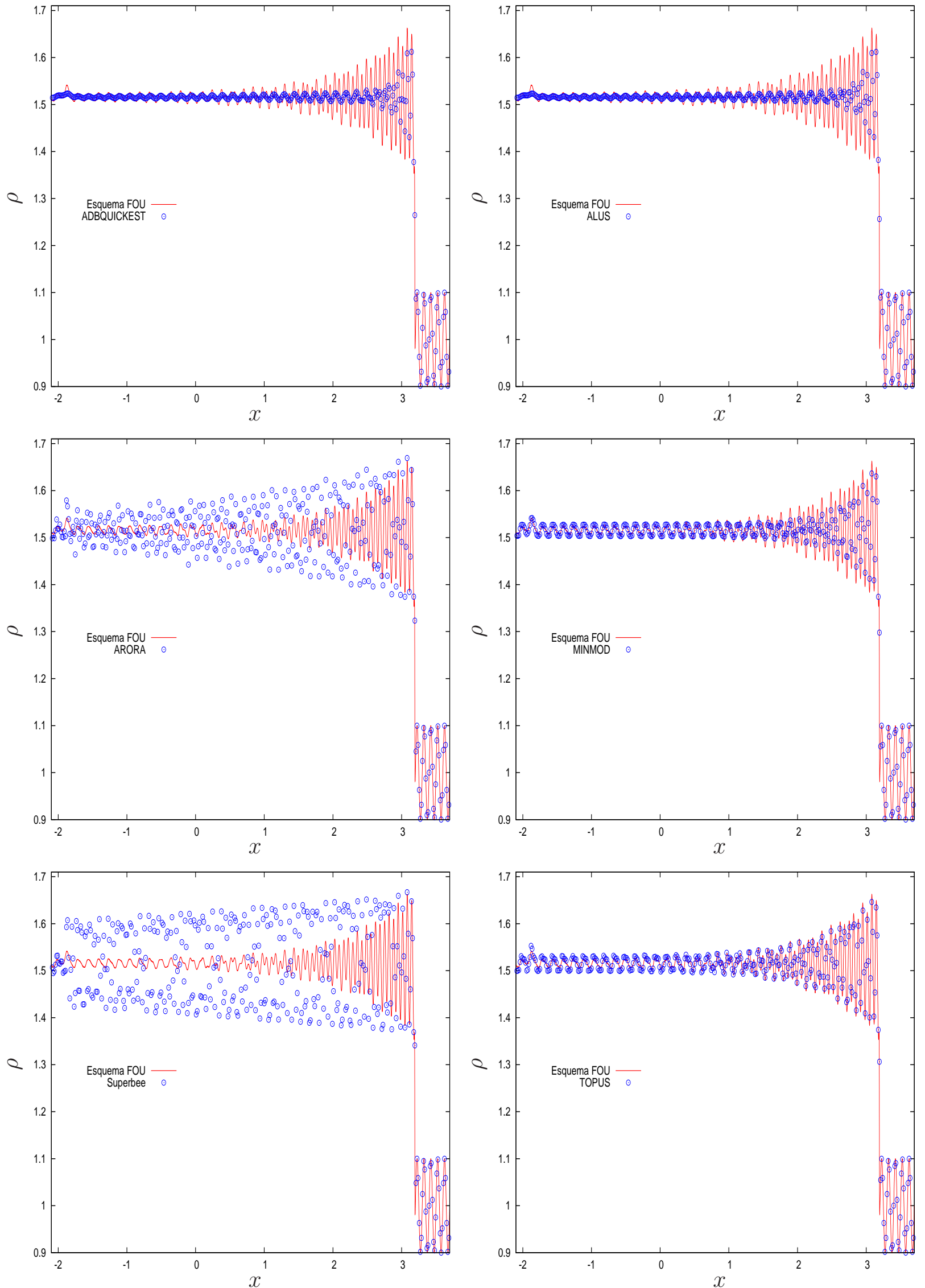

Figura 5.19: Soluções numéricas do tubo de choque de Shu-Osher modificado obtidas com os esquemas ADBQUICKEST, ALUS, ARORA, MINMOD, Superbee e TOPUS. 

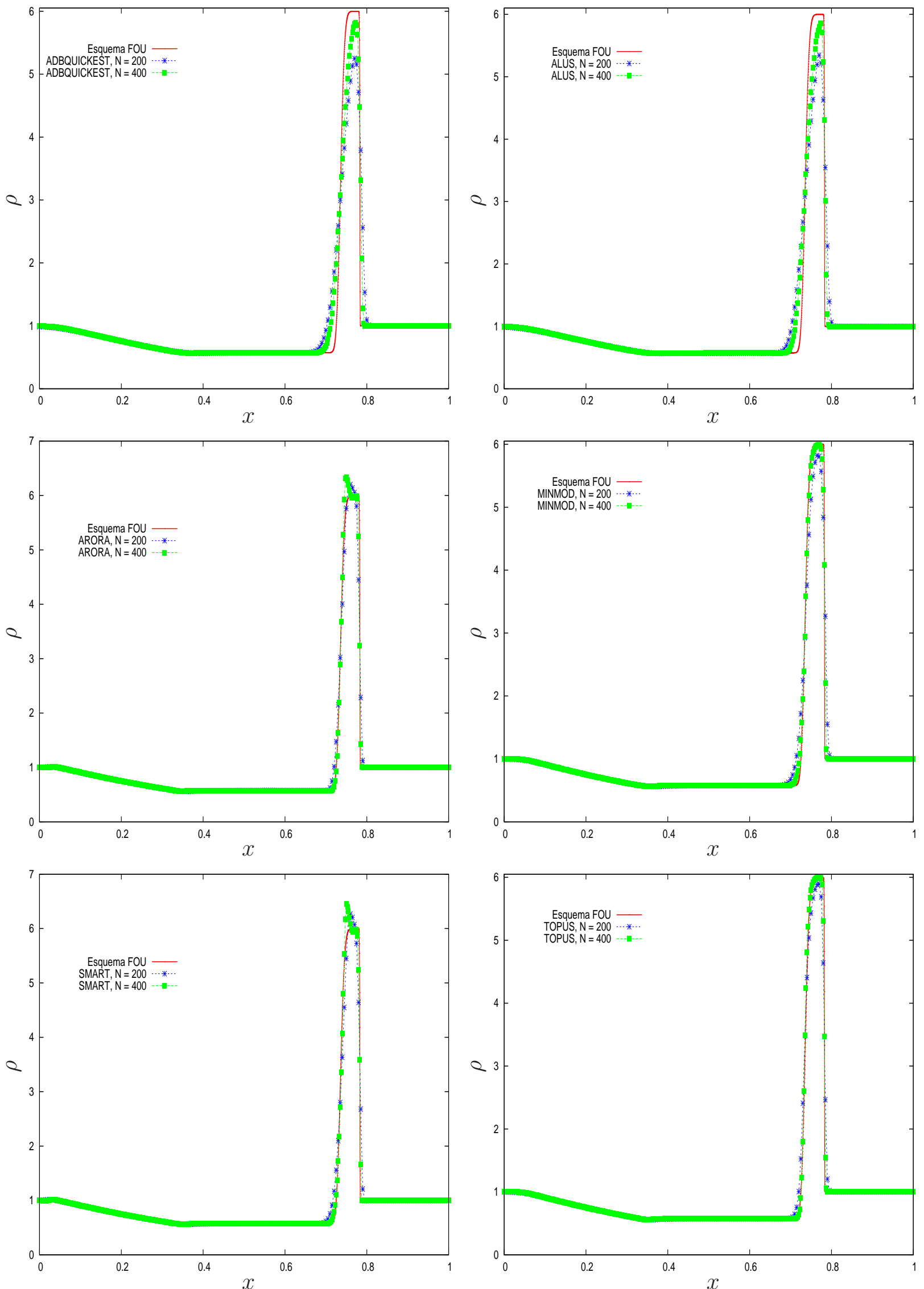

Figura 5.20: Caso 1: Soluções numéricas do tubo de choque de Toro obtidas com os esquemas ADBQUICKEST, ALUS, ARORA, MINMOD, SMART e TOPUS. 

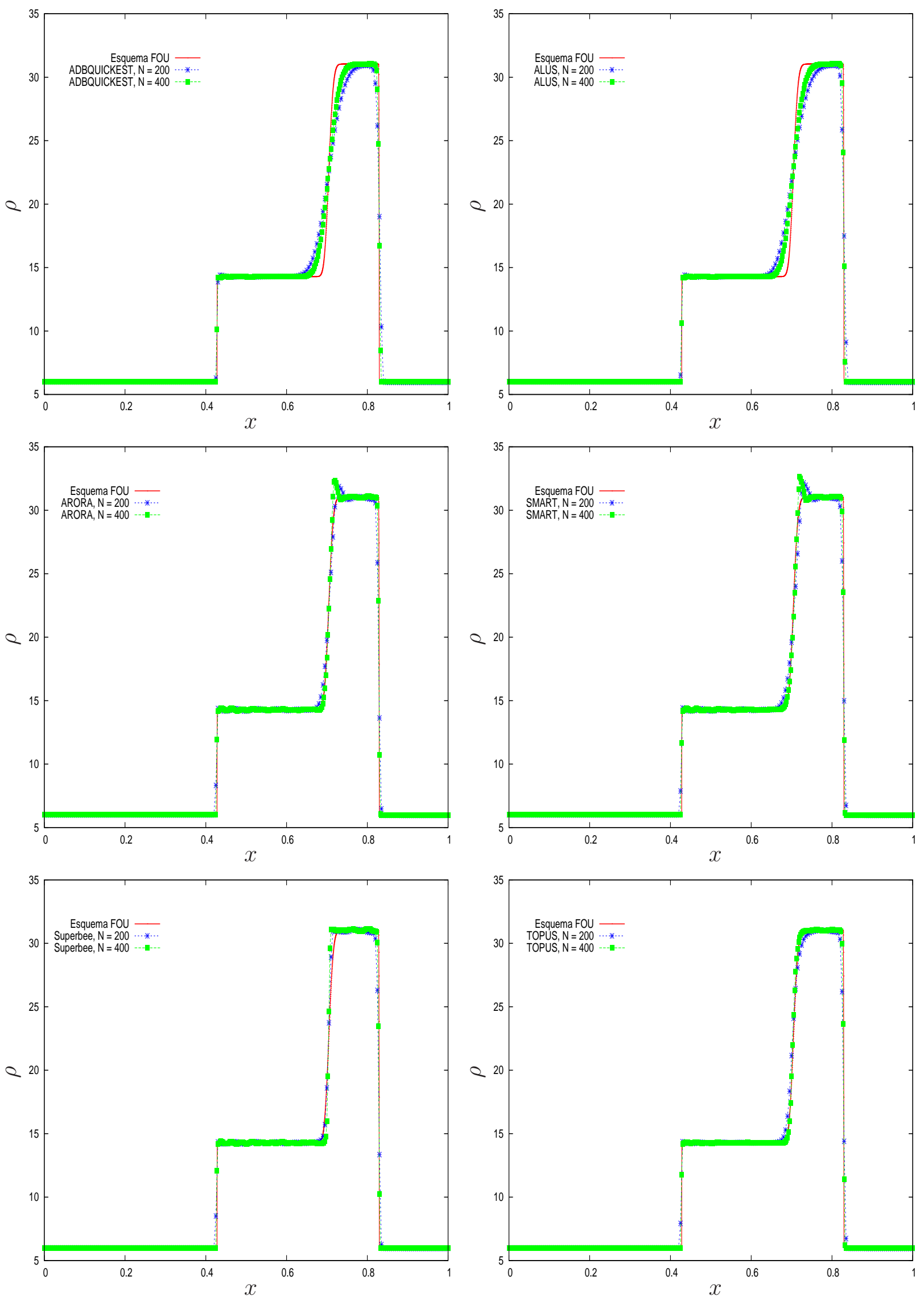

Figura 5.21: Caso 2: Soluções numéricas do tubo de choque de Toro obtidas com os esquemas ADBQUICKEST, ALUS, ARORA, SMART, Superbee e TOPUS. 


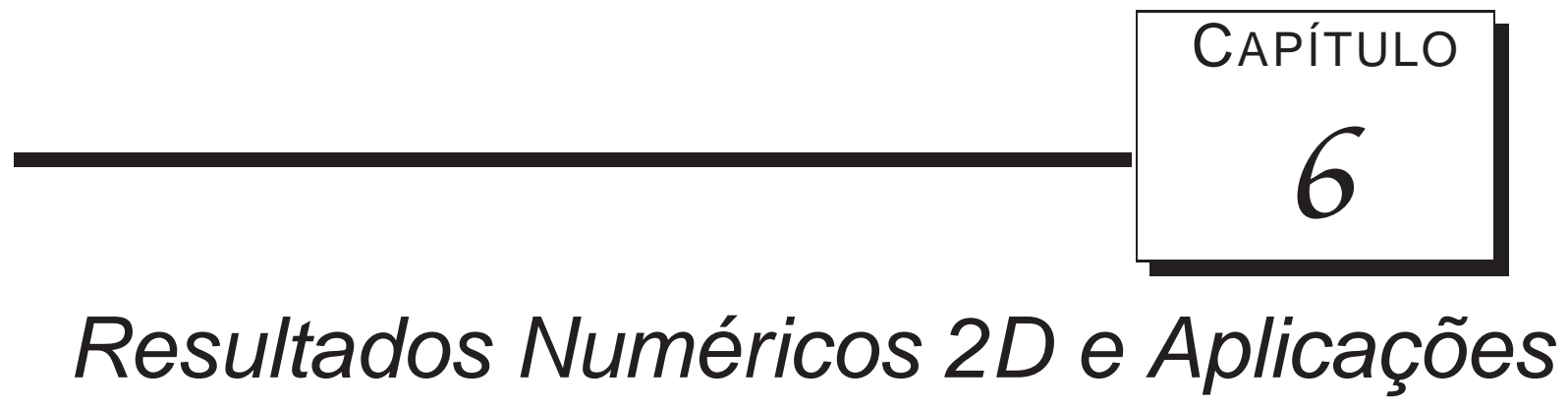

Até este ponto vários problemas 1D tem sido simulados, com o objetivo de verificar a validade, a flexibilidade e a robustez dos esquemas "upwind" propostos nesta dissertação. Neste capítulo, problemas de escoamentos de fluidos 2D complexos são simulados com o uso dos esquemas ALUS e TOPUS, entre outros. Ambas as formulações incompressível e compressível são consideradas.

\subsection{Problemas Incompressíveis}

Nesta seção são apresentados resultados numéricos de uma variedade de problemas de escoamentos de fluidos incompressíveis 2D com ou sem superfícies livres. O código computacional "Freeflow" (versão 2D) de Castelo et al. [20] foi usado nas simulações desses problemas.

\subsubsection{Escoamento de Poiseuille em Regime Laminar}

Para a simulação desse problema confinado, os esquemas ADBQUICKEST, ALUS, CUBISTA e TOPUS foram utilizados. Em particular, as soluções numéricas obtidas nas simulações com o esquema ADBQUICKEST foram parcialmente publicadas em periódico (ver [48]). O problema consiste do escoamento transiente entre duas placas planas situadas em $y=0.0 \mathrm{~m}$ e $y=L=1.0 \mathrm{~m}$. O domínio computacional é $5 L \times L$. Inicialmente, o fluido é movimentado por um gradiente de pressão, gradualmente, ele escoa através do canal e, finalmente, atinge o regime estacionário. A solução semi-analítica (ver [91, 140]) para a componente $u$ da velocidade é definida por

$$
u=-4 y(y-1)-\frac{32}{\pi^{3}} \sum_{n=0}^{N t}(2 n+1)^{3} \sin (\pi y(2 n+1)) \exp \left(-R e^{-1}(2 n+1)^{2} \pi^{2} t\right),
$$

em que $x$ é a coordenada na direção das linhas de corrente do escoamento, $y$ é a coordenada na direção normal ao canal e $N t$ é o número de termos da série. Para as comparações com os dados numéricos, foram usados $N t=1000$ termos na série (6.1). O código "Freeflow-2D" equipado com os esquemas ADBQUICKEST, ALUS, CUBISTA e TOPUS e o método de Crank-Nicolson [26] para a marcha no tempo rodou esse problema usando os seguintes dados: três malhas - $100 \times 20$ $(\delta x=\delta y=0.05 \mathrm{~m}), 200 \times 40(\delta x=\delta y=0.025 \mathrm{~m})$ e $400 \times 80(\delta x=\delta y=0.0125 \mathrm{~m})$; tempo de processamento $t=20 \mathrm{~s}$; e números de Reynolds $R e=0.01$ e $R e=500$. No contorno de 
entrada do fluido, a condição inicial foi calculada por (6.1). A Figura 6.1 mostra, no caso do número de Reynolds ser $R e=0.01$ e a malha de $100 \times 20$ células, a comparação entre as soluções semi-analíticas para a velocidade $u$ em $x=\delta x$ e as soluções numéricas nos tempos $t=0.000157 \mathrm{~s}$, $t=0.0005 \mathrm{~s}, t=0.001 \mathrm{~s}$ e $t=4 \mathrm{~s}$. Observa-se por essa figura que os resultados numéricos obtidos com os esquemas ADBQUICKEST, ALUS, CUBISTA e TOPUS concordam muito bem com as soluções semi-analíticas. Os contornos da componente de velocidade $u$, no tempo $t=20 \mathrm{~s}$, fornecidos com a simulação do esquema TOPUS em três diferentes malhas estão ilustrados na Figura 6.2. Esses contornos resultantes com os esquemas ADBQUICKEST, ALUS, CUBISTA mostram ser qualitativamente semelhantes aos obtidos com o TOPUS e, por esse motivo, foram omitidos.

Com o objetivo de se estimar a ordem de convergência dos esquemas simulados, calcularam-se os erros $\|E\|_{1}$ e $\|E\|_{2}$. A Tabela 6.1 apresenta esses erros e as estimativas da ordem. Pode-se concluir pelos resultados dessa tabela que a segunda ordem de convergência foi praticamente atingida. Nota-se, ainda, que o esquema TOPUS apresentou melhores resultados na malha mais grosseira.
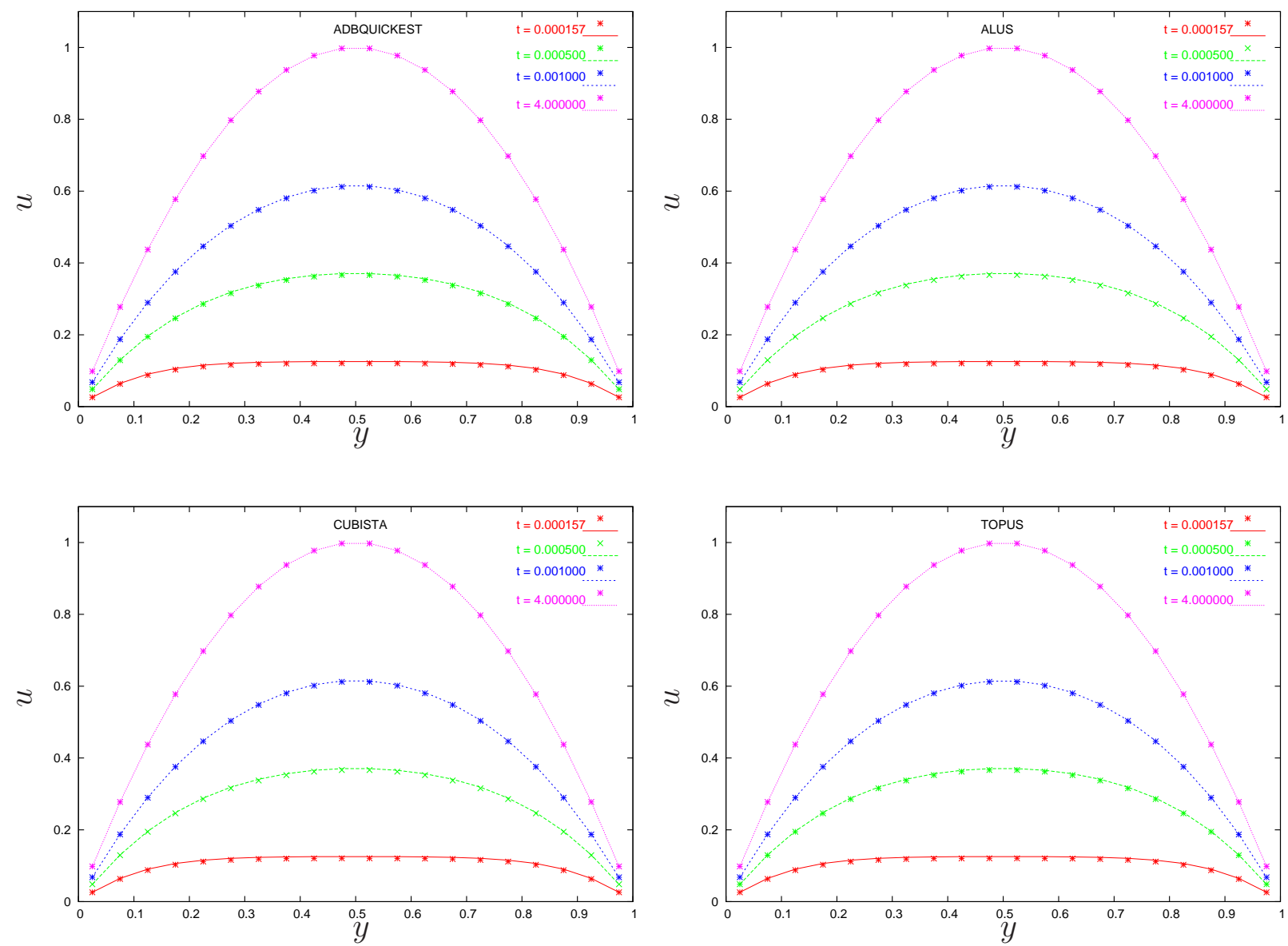

Figura 6.1: Comparação entre as soluções numéricas ("estrelas") e semi-analíticas ("linhas contínuas") para o escoamento de Poiseuille. 
(a) $100 \times 20$ células

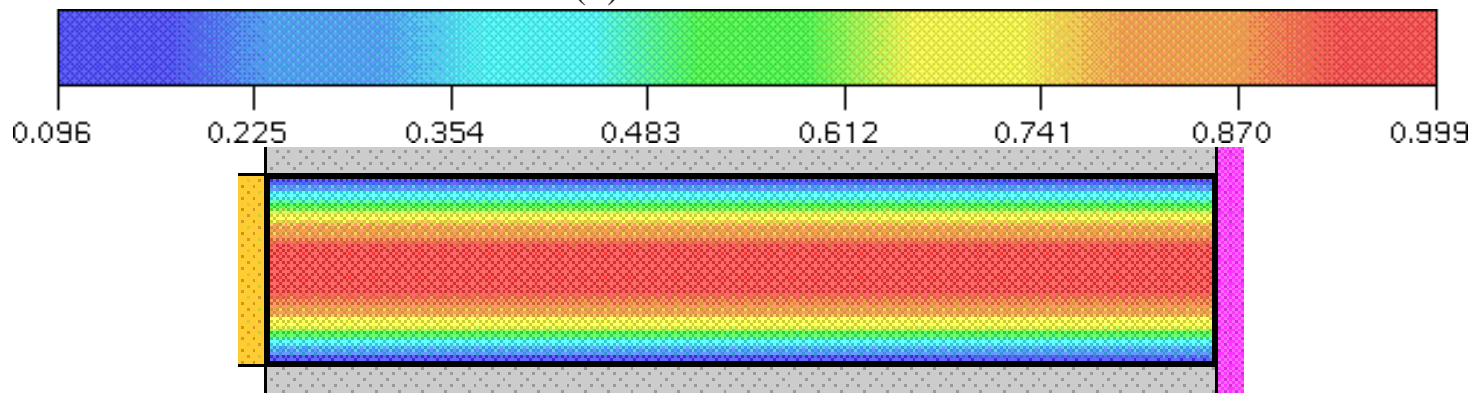

(b) $200 \times 40$ células

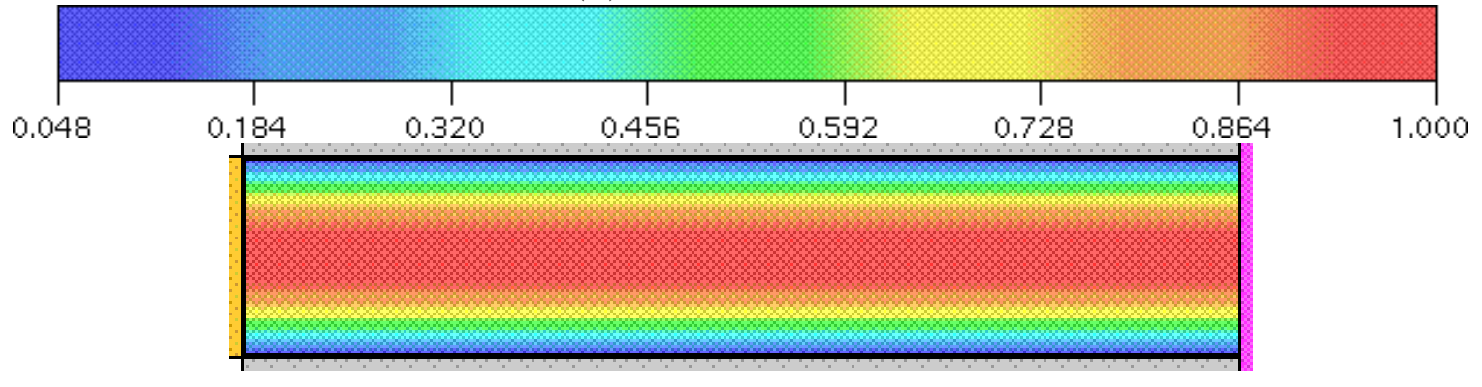

(c) $400 \times 80$ células

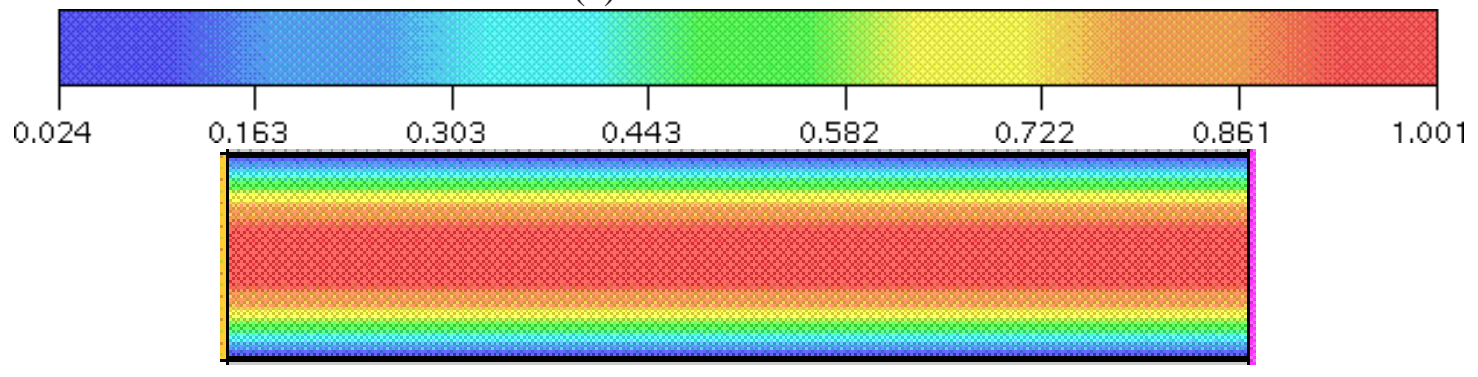

Figura 6.2: Resultados numéricos para a velocidade $u$ obtidos nas simulações do escoamento de Poiseuille usando o esquema TOPUS e três malhas diferentes a Reynolds $R e=500$. 
Tabela 6.1: Estudo da convergência dos esquemas de alta resolução, relacionados com o método implícito de Crank-Nicolson, aplicados ao problema de Poiseuille a Reynolds $R e=500$.

\begin{tabular}{lccccc}
\hline & & Erro & Ordem & Erro & Ordem \\
Esquema & Malha & $\|E\|_{1}$ & $\|E\|_{1}$ & $\|E\|_{2}$ & $\|E\|_{2}$ \\
\hline \multirow{3}{*}{ ADBQUICKEST } & $100 \times 20$ & $8.963 \times 10^{-4}$ & - & $3.654 \times 10^{-4}$ & - \\
& $200 \times 40$ & $2.333 \times 10^{-4}$ & 1.942 & $1.327 \times 10^{-4}$ & 1.461 \\
& $400 \times 80$ & $7.588 \times 10^{-5}$ & 1.620 & $4.165 \times 10^{-5}$ & 1.672 \\
\hline \multirow{2}{*}{ ALUS } & $100 \times 20$ & $9.132 \times 10^{-4}$ & - & $3.726 \times 10^{-4}$ & - \\
& $200 \times 40$ & $2.339 \times 10^{-4}$ & 1.965 & $1.329 \times 10^{-4}$ & 1.487 \\
& $400 \times 80$ & $7.576 \times 10^{-5}$ & 1.626 & $4.166 \times 10^{-5}$ & 1.674 \\
\hline \multirow{2}{*}{ CUBISTA } & $100 \times 20$ & $9.047 \times 10^{-4}$ & - & $3.694 \times 10^{-4}$ & - \\
& $200 \times 40$ & $2.334 \times 10^{-4}$ & 1.955 & $1.328 \times 10^{-4}$ & 1.476 \\
TOPUS & $400 \times 80$ & $7.580 \times 10^{-5}$ & 1.623 & $4.165 \times 10^{-5}$ & 1.673 \\
& $100 \times 20$ & $9.168 \times 10^{-4}$ & - & $3.748 \times 10^{-4}$ & - \\
& $200 \times 40$ & $2.340 \times 10^{-4}$ & 1.970 & $1.330 \times 10^{-4}$ & 1.495 \\
& $400 \times 80$ & $7.608 \times 10^{-5}$ & 1.621 & $4.178 \times 10^{-5}$ & 1.671 \\
\hline
\end{tabular}

\subsubsection{Escoamento numa Expansão Brusca}

Nesta seção são apresentados resultados numéricos para o problema do escoamento confinado 2D numa expansão brusca ${ }^{1}$ [138] nos regimes laminar e turbulento. Esses resultados foram parcialmente publicados em anais de eventos (ver referência [111, 113]).

\section{Caso Laminar}

Os dados numéricos aqui apresentados foram obtidos com os esquemas ADBQUICKEST, ALUS, CDS, CUBISTA, FOU, TOPUS, VONOS e WACEB. A geometria do problema, cujo domínio é $4 m \times 0.2 m$, está ilustrada na Figura 6.3. O objetivo principal nesse problema consiste em estimar o comprimento da recirculação principal $x r$, que é determinada como sendo o ponto sobre a primeira seqüência de células adjacentes ao contorno rígido inferior, em que a componente $u$ da velocidade troca de sinal.

Os dados do modelo utilizado nas simulações são o diâmetro do injetor $h=0.1 \mathrm{~m}$, a velocidade de injeção $U_{0}=1 \mathrm{~ms}^{-1}$ e o parâmetro de escala $L=0.2 \mathrm{~m}$. Conforme Brandi [17], as simulações deste escoamento confinado foram realizadas a Reynolds $R e=100$, 200, 400, 600 e 800 numa malha de $400 \times 20(\delta x=\delta y=0.01 m)$ células computacionais. Na entrada do canal é imposto um perfil parabólico definido por

$$
u(y)=\frac{-4 U}{h^{2}} y^{2}+\frac{4 U}{h} y .
$$

Os resultados numéricos para a recirculação $x r$, usando os vários esquemas e os dados experimentais de Armaly et al. [8], estão mostrados na Tabela 6.2. Nota-se por essa tabela que os resultados numéricos obtidos com os esquemas estão em boa concordância com os dados experimentais. Também, percebe-se que a estimativa para a recirculação com o esquema CDS é melhor, quando comparada com os outros esquemas, e as estimativas com o ALUS e TOPUS ficaram próximas daquela obtida com o esquema centrado. Os contornos da componente de velocidade $u$, no tempo

\footnotetext{
${ }^{1}$ Este problema é conhecido na literatura por "backward facing step".
} 
$t=100 \mathrm{~s}$, a Reynolds 100 e 800 estão mostrados, respectivamente, nas Figuras 6.4 e 6.5. Dessas figuras, é possível observar que com o aumento do número de Reynolds implicou num aumento da recirculação $x r$ e aparecimento de uma outra recirculação no topo do canal para $R e=800$. Ressalta-se que esses resultados estão, qualitativamente, consistentes com os dados dos trabalhos de Brandi [17] e de Stuart e Dochan [138].

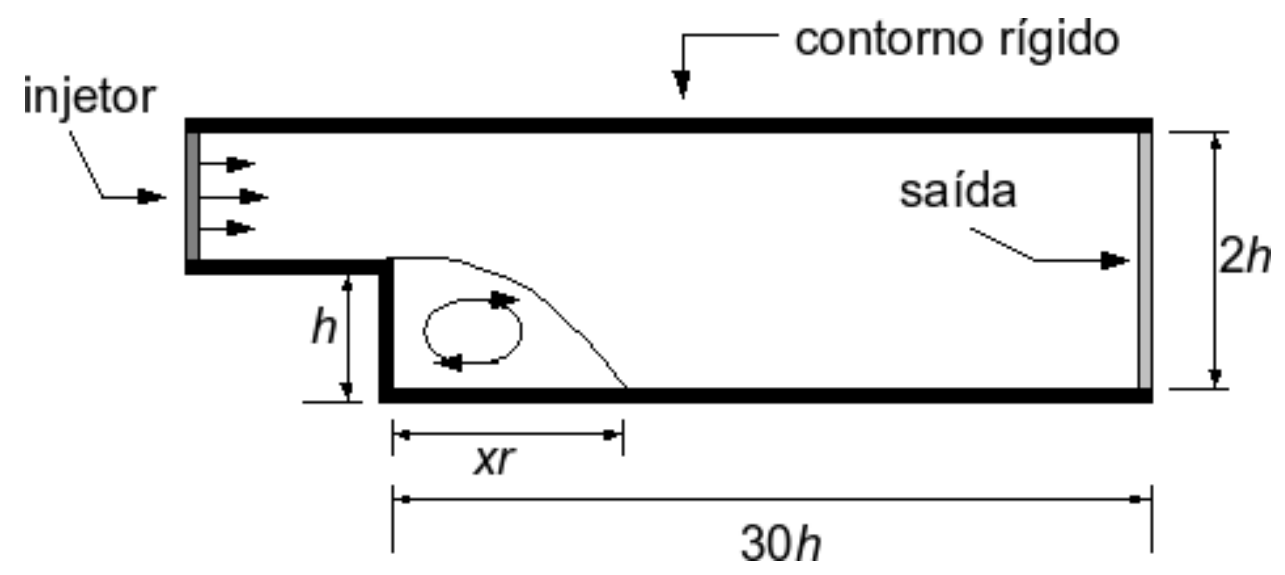

Figura 6.3: Geometria para o problema confinado numa expansão brusca.

Tabela 6.2: Comparação entre resultados experimentais e numéricos obtidos para o problema confinado numa expansão brusca usando diferentes esquemas convectivos e números de Reynolds.

\begin{tabular}{|cccccc|}
\hline \hline & \multicolumn{5}{c|}{$\mathrm{Re}$} \\
\cline { 2 - 6 } & 100 & 200 & 400 & 600 & 800 \\
\hline Experimental [8] & 3.06 & 5.16 & 8.72 & 11.28 & 14.34 \\
ADBQUICKEST & 3.137 & 5.140 & 8.141 & 9.731 & 10.713 \\
ALUS & 3.135 & 5.181 & 8.382 & 9.973 & 10.691 \\
CDS & 3.138 & 5.213 & 8.561 & 10.358 & 10.997 \\
CUBISTA & 3.133 & 5.139 & 8.151 & 9.710 & 10.666 \\
FOU & 2.749 & 4.337 & 6.942 & 8.906 & 10.230 \\
TOPUS & 3.128 & 5.145 & 8.224 & 9.827 & 10.777 \\
VONOS & 3.163 & 5.148 & 8.016 & 9.553 & 10.576 \\
WACEB & 3.137 & 5.151 & 8.200 & 9.799 & 10.735 \\
\hline \hline
\end{tabular}

Além disso, um teste de convergência das soluções numéricas, para a componente $u$, foi feito com Reynolds 600 em três malhas consistindo de $200 \times 10,400 \times 20$ e $800 \times 40$ células computacionais. Esses dados estão mostrados na Figura 6.6, onde se observa como foi calculado o comprimento de recirculação ${ }^{2}(x r)$. Pode-se observar nessa figura que o maior valor de $x g$, e portanto o maior $x r$, é obtido quando se utiliza a malha mais fina. Comparando-se o valor de $x r$ com aqueles da Tabela 6.2, que foram obtidos numa malha de $400 \times 20$ células, fica claro que a solução numérica se aproxima do dado experimental $(x r=11.28)$ com o refinamento da malha.

\footnotetext{
${ }^{2}$ Neste trabalho, tem-se $x r=(x g-1) / 0.1$, em que $x g$ é o ponto onde a velocidade troca de sinal.
} 
(a) ADBQUICKEST

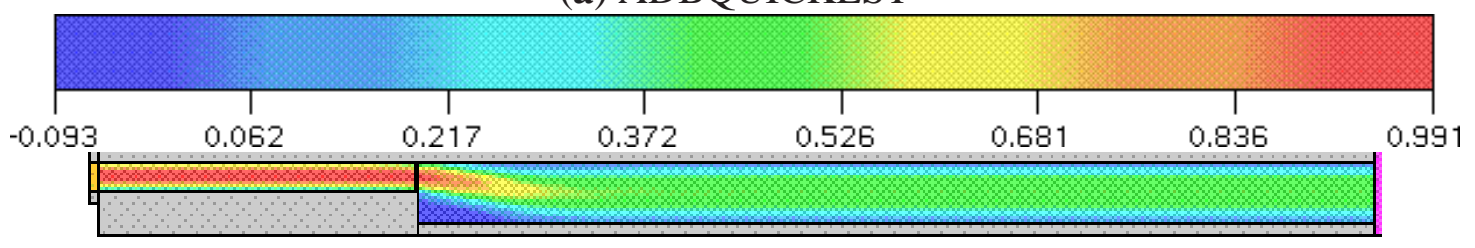

(b) ALUS

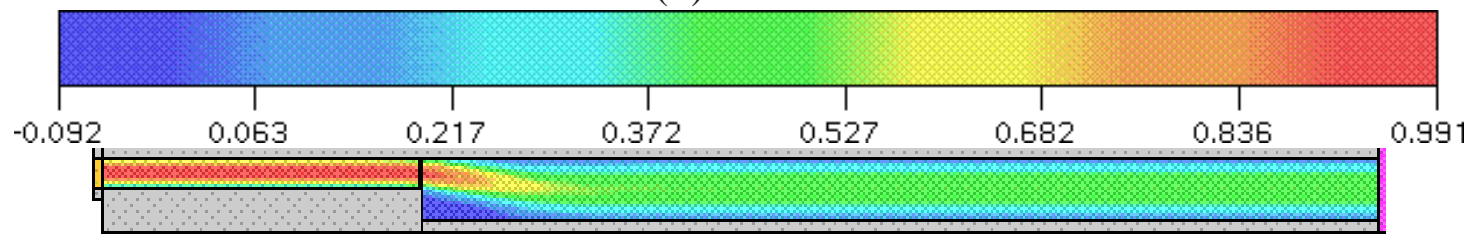

(c) CDS

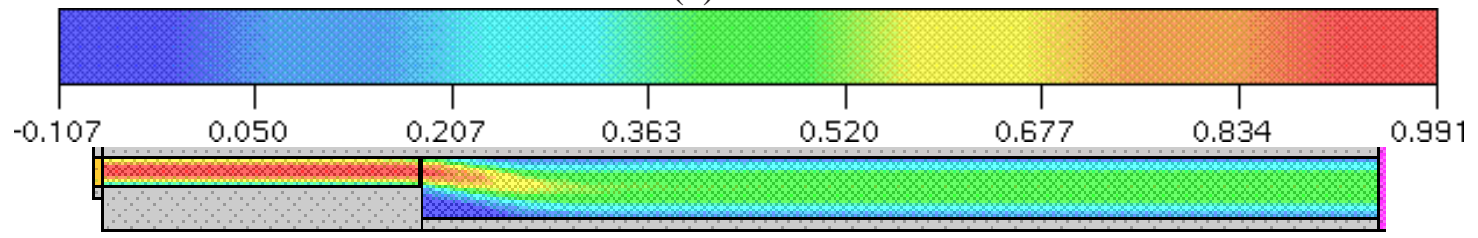

(d) CUBISTA

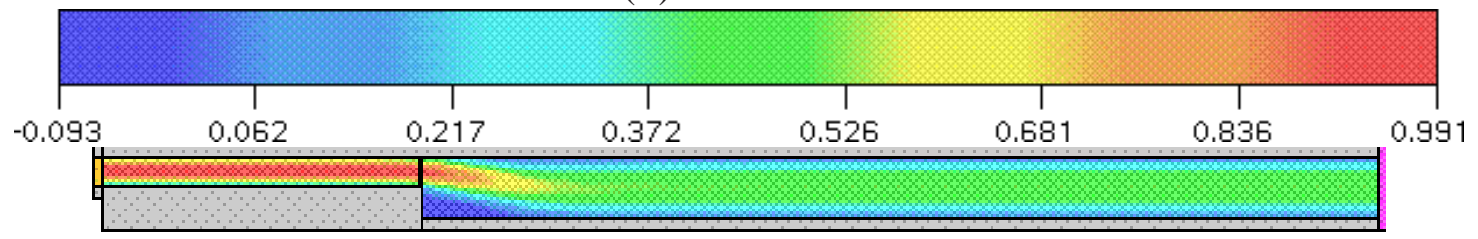

(e) FOU

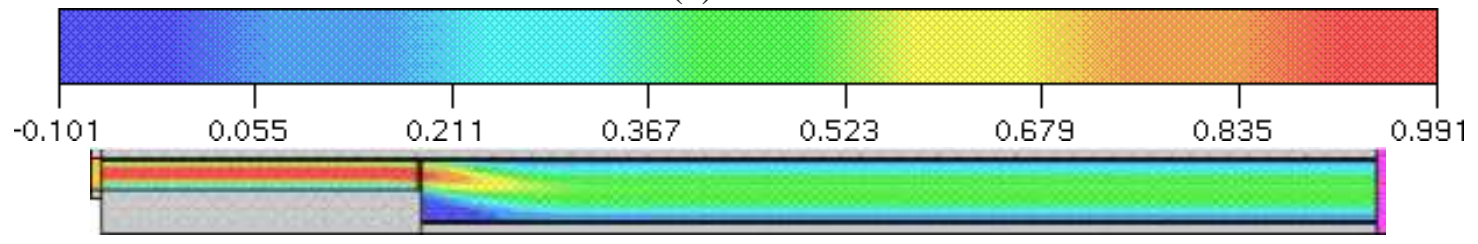

(f) TOPUS

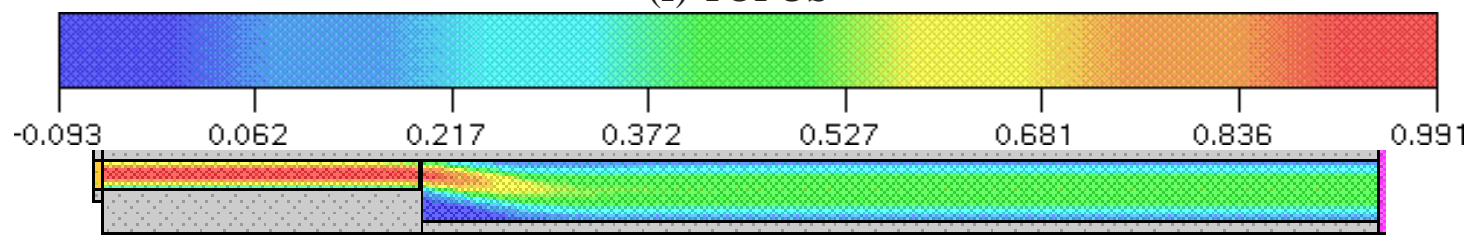

(g) VONOS

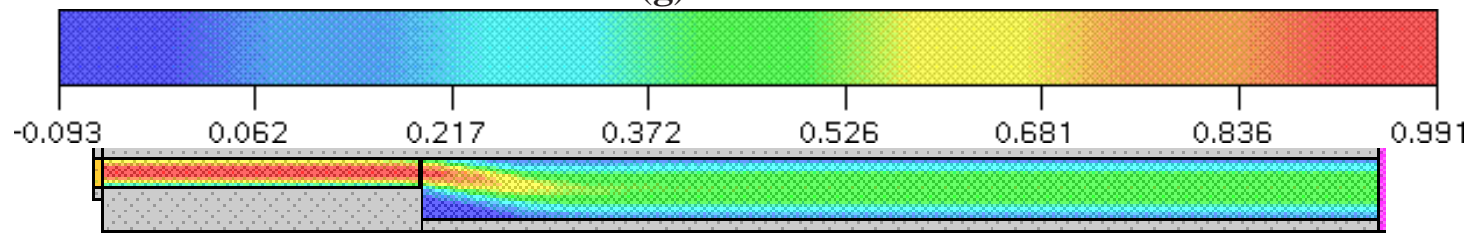

(h) WACEB

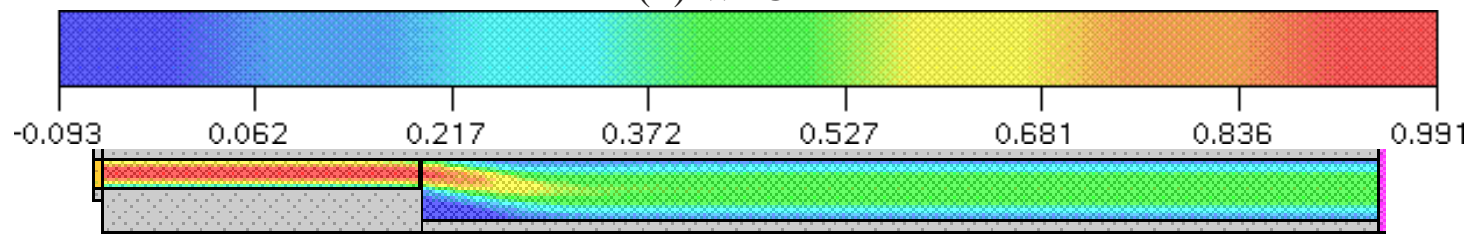

Figura 6.4: Resultados numéricos para a velocidade $u$ obtidos nas simulações do escoamento numa expansão brusca a Reynolds $R e=100$. 
(a) ADBQUICKEST

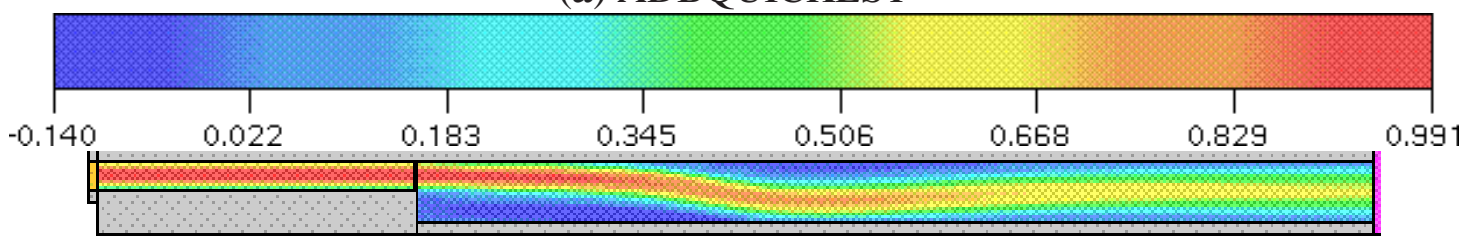

(b) ALUS

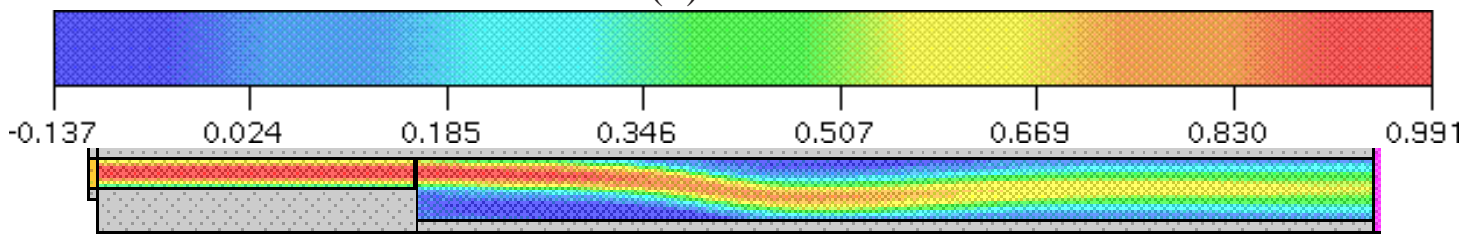

(c) $\mathrm{CDS}$

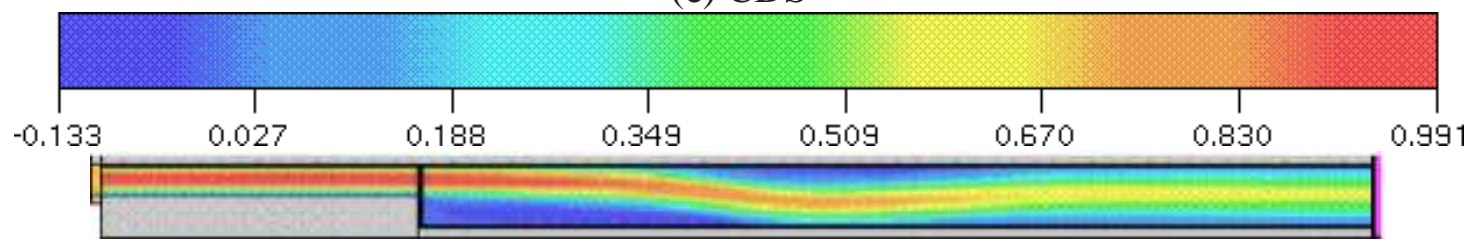

(d) CUBISTA

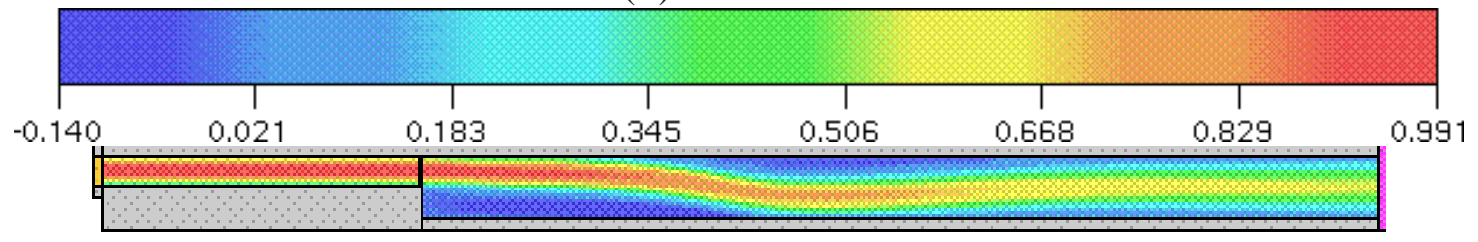

(e) FOU

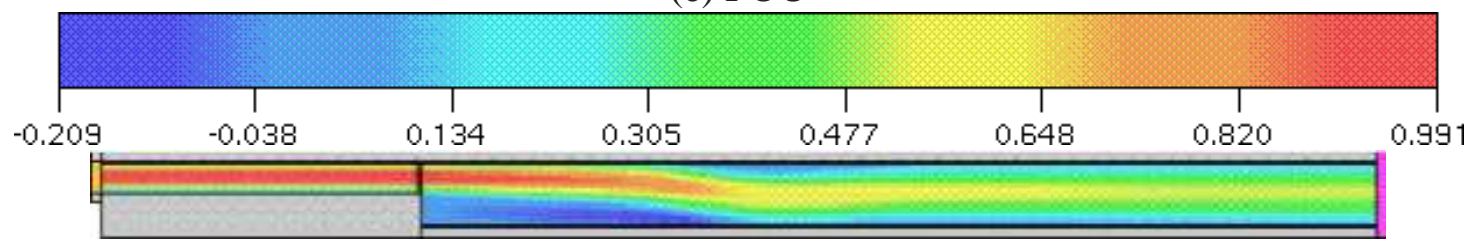

(f) TOPUS

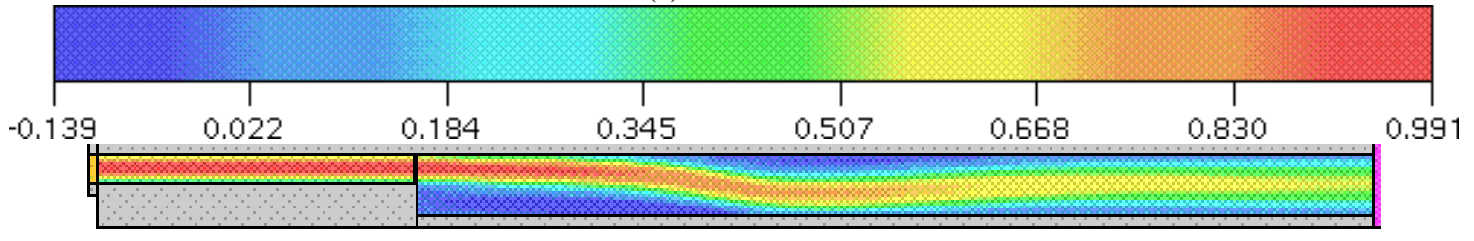

(g) VONOS

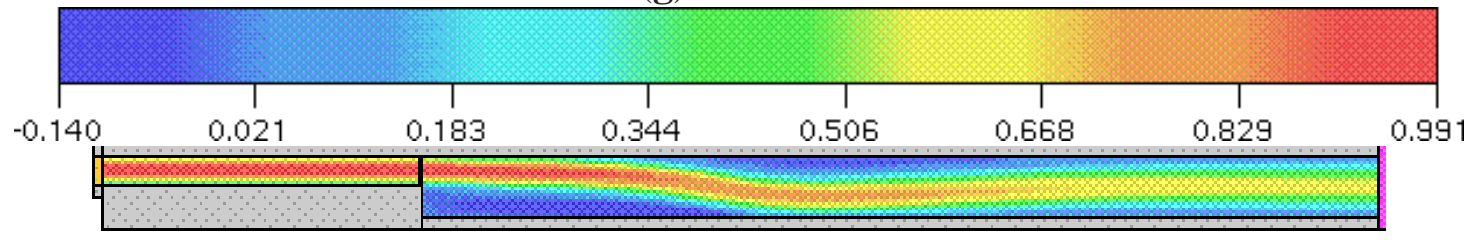

(h) WACEB

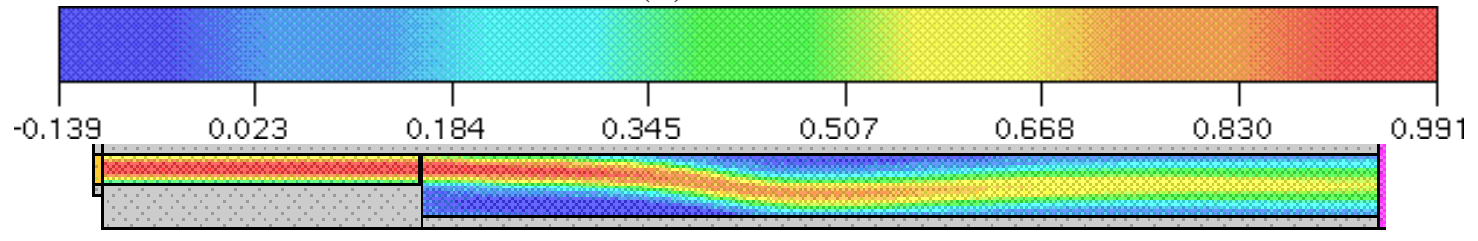

Figura 6.5: Resultados numéricos para a velocidade $u$ obtidos nas simulações do escoamento numa expansão brusca a Reynolds $R e=800$. 
(a) ADBQUICKEST $(x r=10.055)$

(b) ALUS $(x r=10.153)$

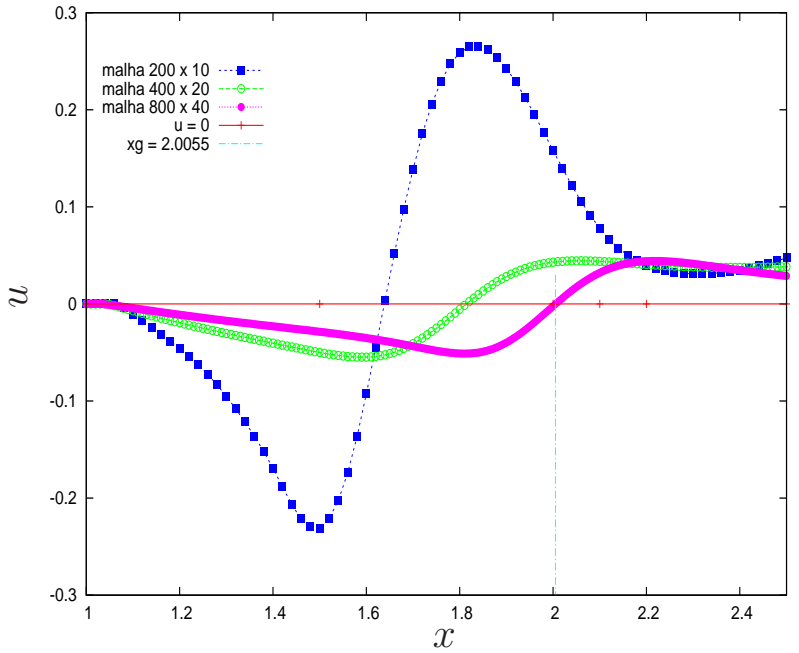

(c) CUBISTA $(x r=10.074)$

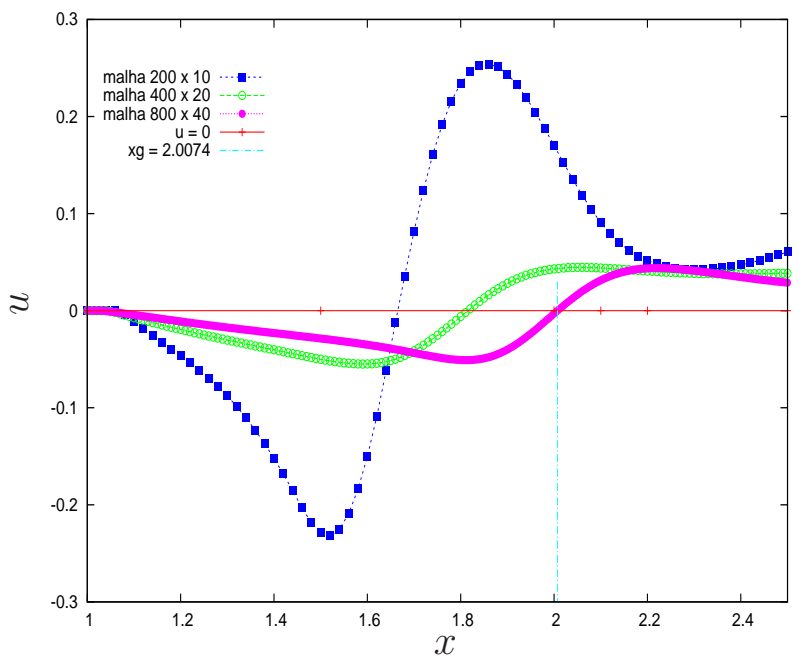

(e) $\operatorname{VONOS}(x r=9.966)$

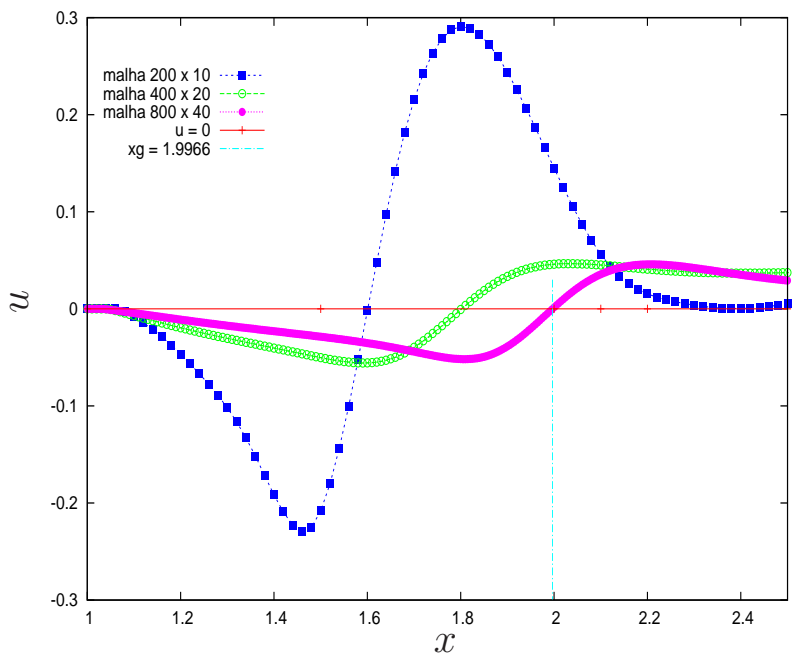

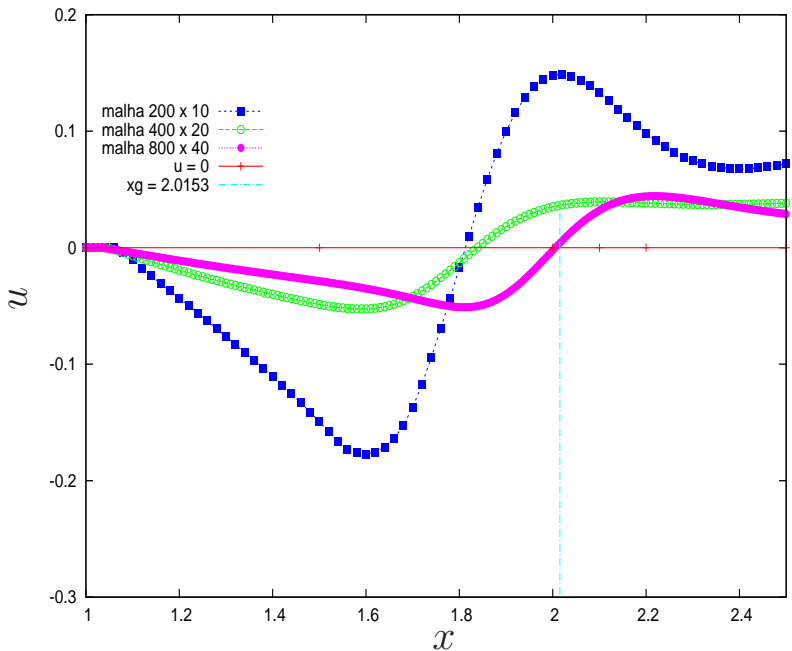

(d) TOPUS $(x r=10.083)$

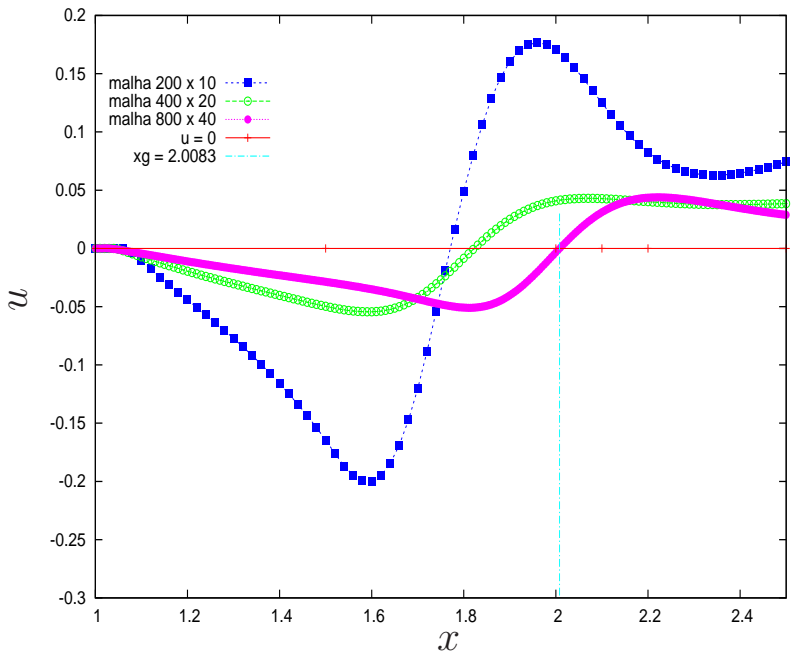

(f) WACEB $(x r=10.080)$

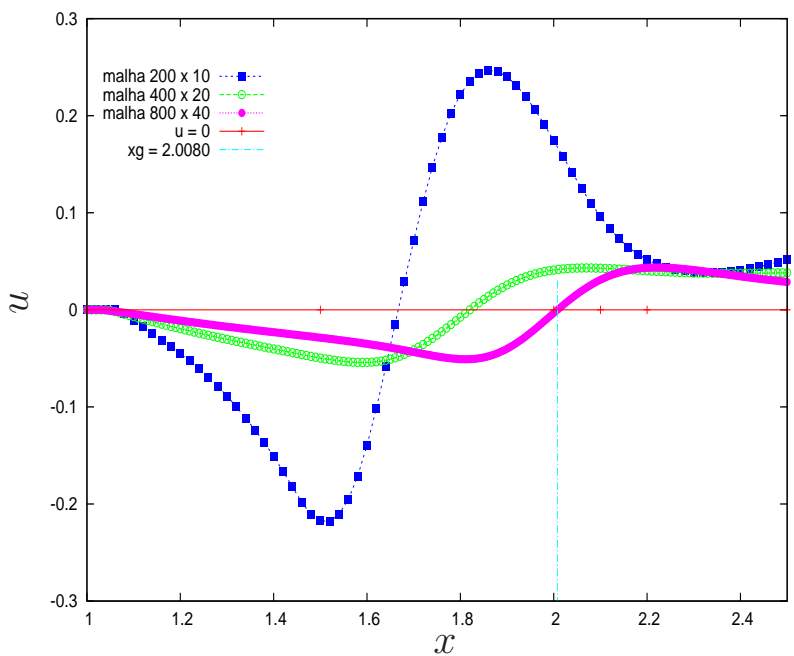

Figura 6.6: Perfil da componente $u$ da velocidade do problema da expansão brusca quando $R e=$ 600, mostrando a melhoria da solução numérica com o refinamento da malha. 


\section{Caso Turbulento}

Também, para a simulação do escoamento numa expansão brusca, foram utilizados os modelos $\kappa-\varepsilon$ clássico e o modelo algébrico RSAEM. Os resultados numéricos aqui apresentados são para esse problema, cujo domínio é $4 m \times 0.3 m$, em regime turbulento. Os esquemas utilizados nas simulações foram os mesmos do caso laminar, com exceção dos esquemas CDS e FOU. Como no caso laminar, o objetivo principal é estimar o comprimento da recirculação principal $x r$. Os dados do modelo simulado são: diâmetro do injetor $h=0.1 \mathrm{~m}$; velocidade de injeção $U=1 \mathrm{~ms}^{-1}$; parâmetro de escala $L=0.3 \mathrm{~m}$; número de Reynolds $R e=1.32 \times 10^{5}$ e três malhas: $200 \times 15$ $(\delta x=\delta y=0.02 m), 400 \times 30(\delta x=\delta y=0.01 m)$ e $800 \times 60(\delta x=\delta y=0.005 m)$ células computacionais.

Tomando-se como base os dados numérico de Tangham e Speziale [136] e experimental de Eaton e Johnston [36], cujos valores da recirculação são $x r=6.0$ e $x r=7.1$, respectivamente; as Tabelas 6.3 e 6.4 apresentam as comparações desses dados com os numéricos obtidos com os modelos de turbulência $\kappa-\varepsilon$ e RSAEM, respectivamente. Observando a primeira tabela, nota-se que o esquema ADBQUICKEST forneceu resultado mais perto do experimental na malha grossa $(200 \times 15$ células) quando comparado com os outros esquemas. Por outro lado, a simulação com o TOPUS na malha fina $(800 \times 60$ células $)$ forneceu um valor mais próximo do experimental. Quanto aos dados apresentados na segunda tabela, nota-se que a combinação da modelagem RSAEM e TOPUS forneceu resultados bastante satisfatórios. Em suma, as Tabelas 6.3 e 6.4 demonstram que os resultados numéricos obtidos com os modelos de turbulência simulados estão em concordância com Speziale e Thangam [136]. Como ilustração, os contornos da componente de velocidade $u$, no tempo $t=100 \mathrm{~s}$, obtidos usando os modelos $\kappa-\varepsilon$ e RSAEM estão mostrados, respectivamente, nas Figuras 6.7 e 6.8.

Tabela 6.3: Valores da recirculação obtidos para o problema de expansão brusca no caso turbulento usando o modelo $\kappa-\varepsilon$.

\begin{tabular}{|cccc|}
\hline \hline & \multicolumn{3}{c|}{ Malha (número de células) } \\
\cline { 2 - 4 } Esquema “upwind" & $200 \times 15$ & $400 \times 30$ & $800 \times 60$ \\
\hline ADBQUICKEST & 6.9783 & 6.1302 & 5.5768 \\
ALUS & 7.7915 & 6.3045 & 5.6214 \\
CUBISTA & 7.2878 & 6.1952 & 5.5939 \\
TOPUS & 7.9848 & 6.6994 & 6.1756 \\
VONOS & 6.2253 & 5.7561 & 5.4209 \\
WACEB & 7.2263 & 6.1604 & 5.5065 \\
\hline \hline
\end{tabular}


(a) ADBQUICKEST

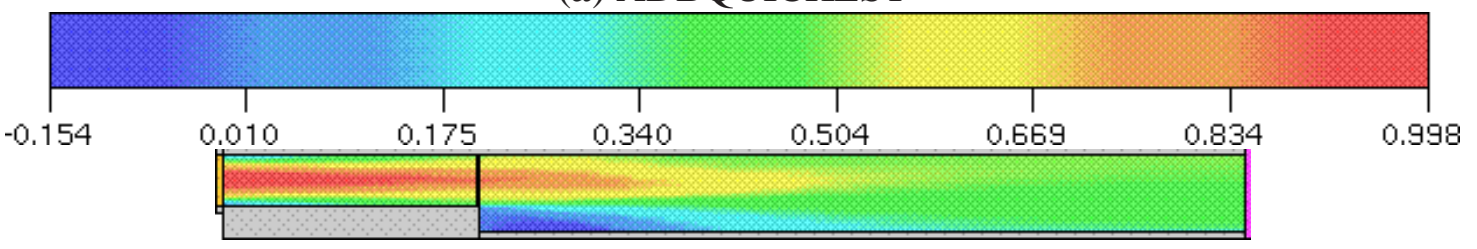

(b) ALUS

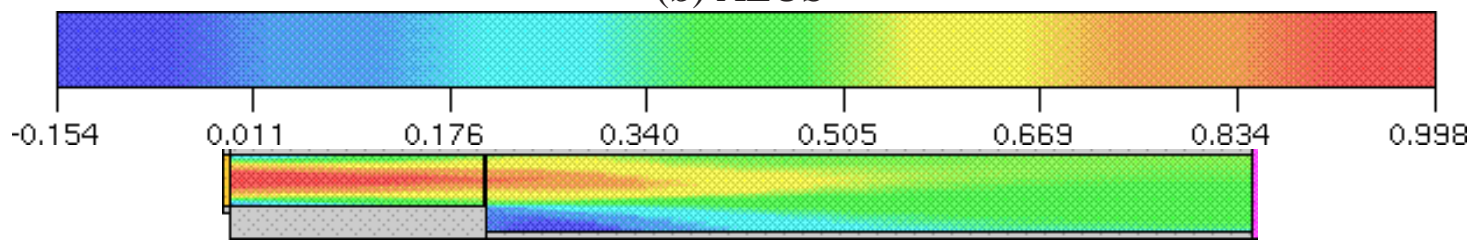

(c) CUBISTA

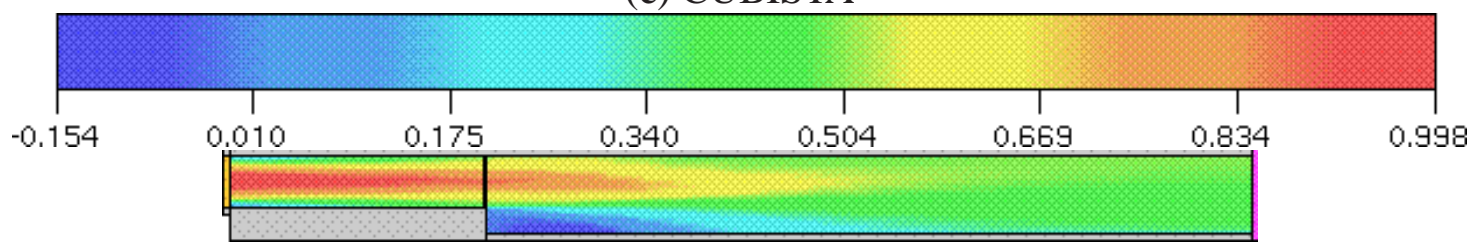

(d) TOPUS

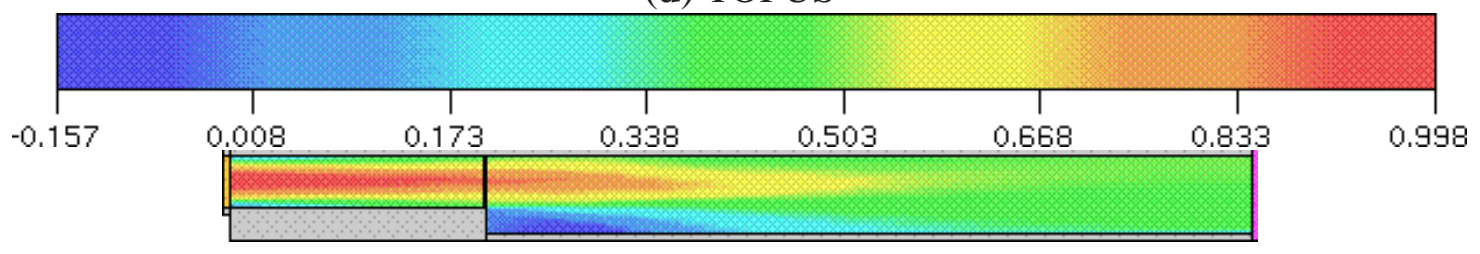

(e) VONOS

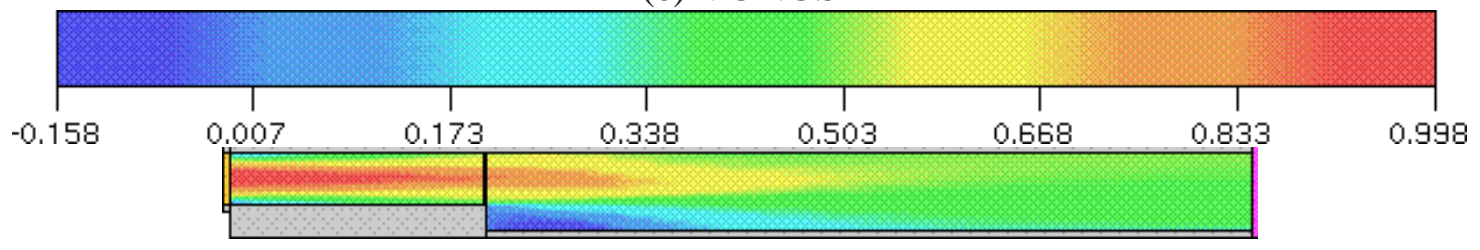

(f) WACEB

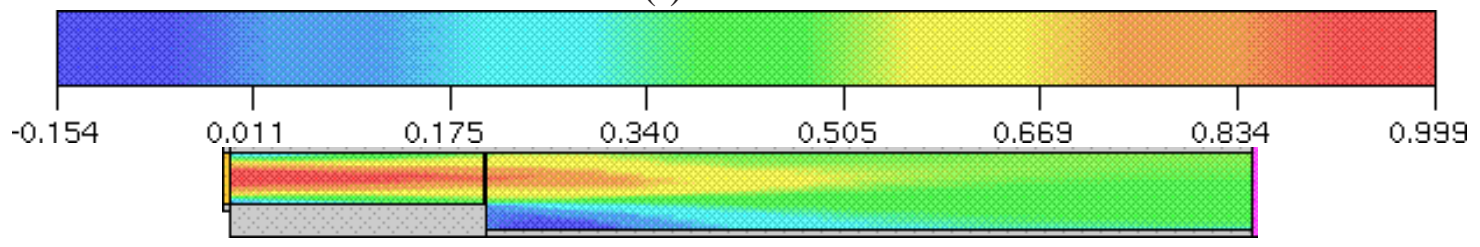

Figura 6.7: Resultados numéricos do campo de velocidade na direção $x$ obtidos nas simulações do problema da expansão brusca usando o modelo $\kappa-\varepsilon$. 
(a) ADBQUICKEST

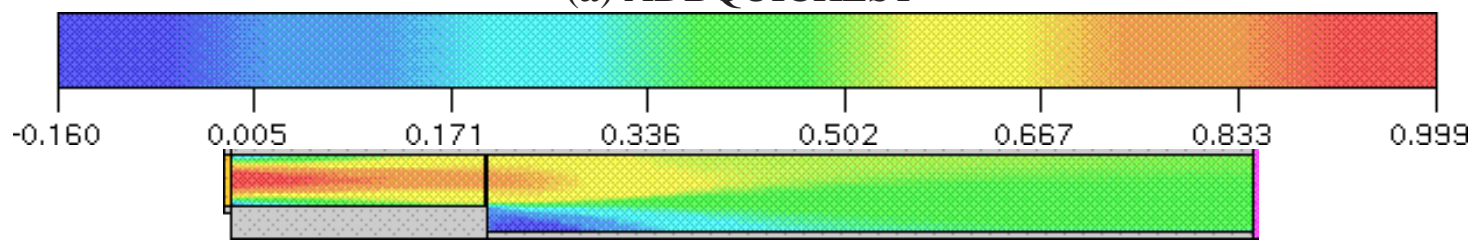

(b) ALUS

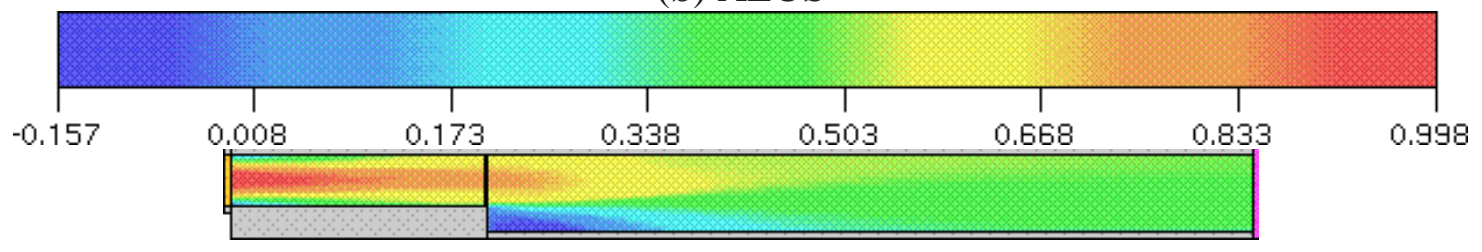

(c) CUBISTA

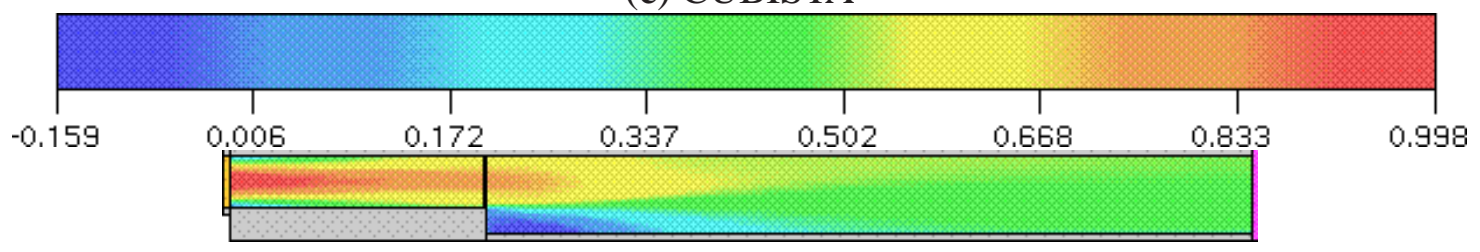

(d) TOPUS

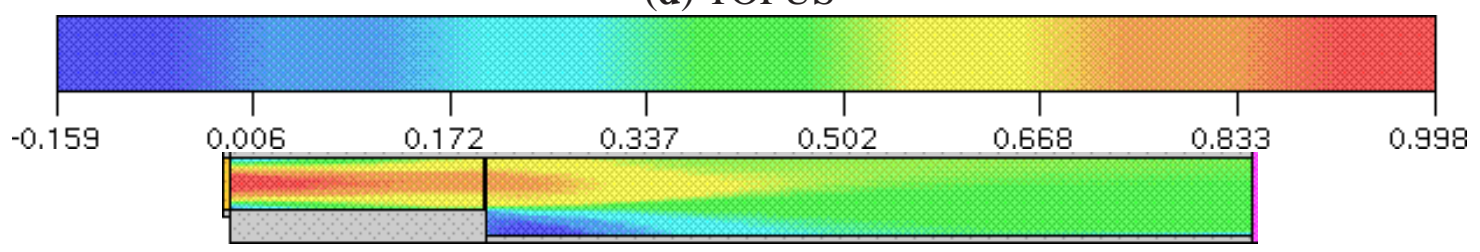

(e) VONOS

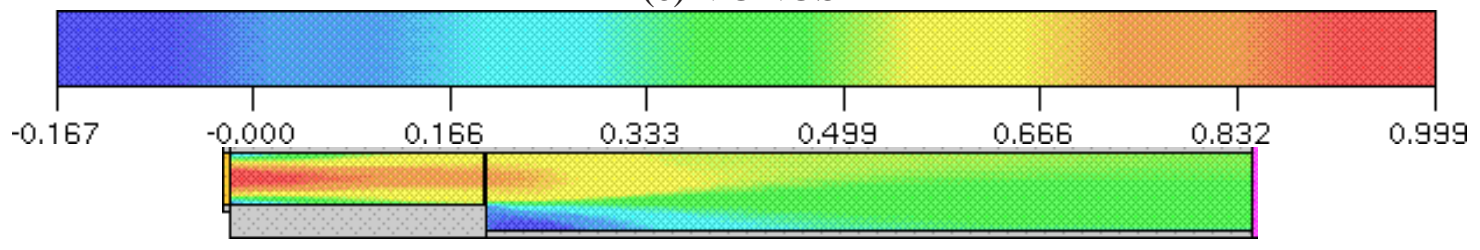

(f) WACEB

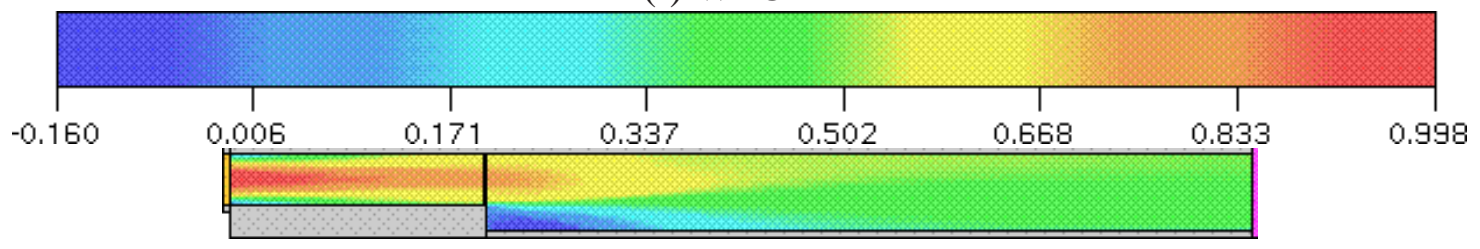

Figura 6.8: Resultados numéricos do campo de velocidade na direção $x$ obtidos nas simulações do problema da expansão brusca usando o modelo algébrico RSAEM. 
Tabela 6.4: Valores da recirculação obtidos para o problema de expansão brusca no caso turbulento usando o modelo RSAEM.

\begin{tabular}{|cccc|}
\hline \hline & \multicolumn{3}{c|}{ Malha (número de células) } \\
\cline { 2 - 4 } Esquema "upwind" & $200 \times 15$ & $400 \times 30$ & $800 \times 60$ \\
\hline ADBQUICKEST & 4.9626 & 4.6576 & 4.5389 \\
ALUS & 5.3447 & 4.7722 & 4.5606 \\
CUBISTA & 5.1222 & 4.7122 & 4.5478 \\
TOPUS & 5.4326 & 4.9411 & 4.8958 \\
VONOS & 4.6680 & 4.4576 & 4.4875 \\
WACEB & 5.0157 & 4.6876 & 4.5440 \\
\hline \hline
\end{tabular}

\subsubsection{Colapso de uma Coluna de Fluido}

Nesta seção são apresentadas as soluções numéricas confrontadas com dados experimentais do problema ${ }^{3}$ do colapso de uma coluna de fluido. Os resultados mostrados nesta seção foram parcialmente publicados em anais de eventos e periódico (ver referências [48, 112, 113]). Esse problema é um teste bastante significativo para validação de métodos numéricos para escoamentos com superfícies livres móveis. Ele consiste de uma coluna de fluido confinado entre paredes, que inicialmente está em repouso, conforme ilustrado na Figura 6.9. Em seguida, uma das paredes é removida subitamente e o fluido fica livre para escoar sob à ação do campo gravitacional. Martin e Moyce [87] foram os primeiros pesquisadores a estudar experimentalmente tal problema em detalhes. Em seguida, Koshizuka e Oka [70] investigaram a distribuição do campo de velocidade e a taxa de colapso da coluna de água. Mais recentemente, o experimento de Martin e Moyce [87] foi repetido com outras técnicas por Stansby et al. [137].

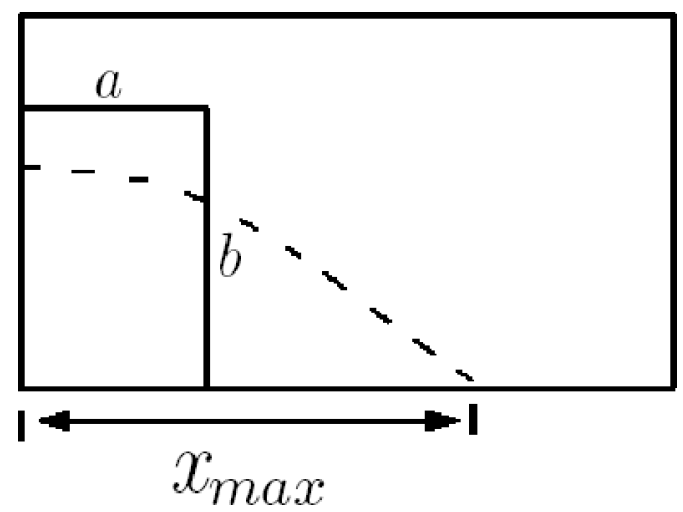

Figura 6.9: Geometria do problema do colapso de uma coluna de fluido: $a=0.05 \mathrm{~m}$ e $b=0.1 \mathrm{~m}$.

As simulações foram feitas sem (direta) e com modelagem da turbulência (modelos $\kappa-\varepsilon$ e RSAEM), ambas associadas com os esquemas ADBQUICKEST, ALUS, CUBISTA e TOPUS. Os seguintes dados foram empregados nas simulações: domínio computacional $0.3 \mathrm{~m} \times 0.15 \mathrm{~m}$ e malha de $150 \times 75$ células $(\delta x=\delta y=0.002 m)$ computacionais. As escalas envolvidas no problema são $L=a$ e $U_{0}=\sqrt{0.4905} \mathrm{~m} / \mathrm{s}$, de maneira que o número de Reynolds é $R e=9.91 \times 10^{4}$. As Figuras 6.11 e 6.12 ilustram, respectivamente, os resultados obtidos com e sem modelagem de turbulência

\footnotetext{
${ }^{3}$ Este problema é conhecido na literatura por "broken dam".
} 
combinados com os esquemas ALUS e TOPUS para a frente do fluido $\left(x_{\max }\right)$ em função do tempo. Os resultados obtidos com os esquemas ADBQUICKEST e CUBISTA foram omitidos, pois eles mostraram-se qualitativamente semelhantes. Como pode ser visto por essas figuras, houve boa concordância entre os dados numéricos e experimentais de Koshizuka e Oka [70] e de Martin e Moyce [87]. A Figura 6.10 ilustra, nos tempos $t=0.05 s, t=0.10 s, t=0.15 \mathrm{~s}$ e $t=0.20 \mathrm{~s}$, os campos de pressão do escoamento.

\section{(a) ALUS}
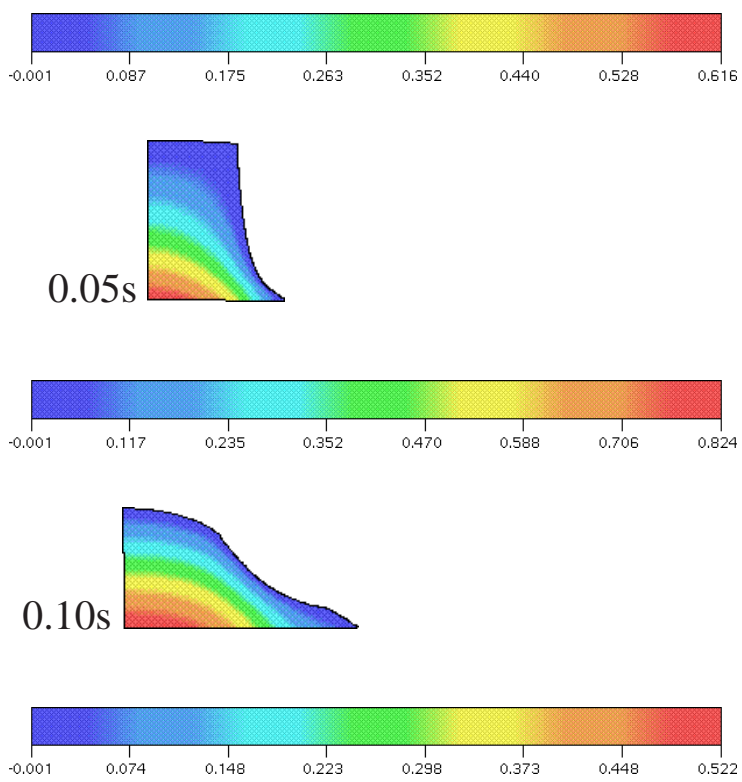

$0.15 \mathrm{~s}$

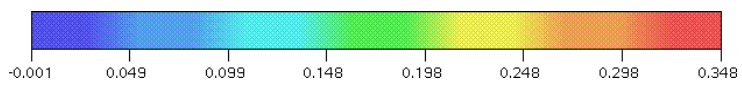

$0.20 \mathrm{~s}$ (b) TOPUS

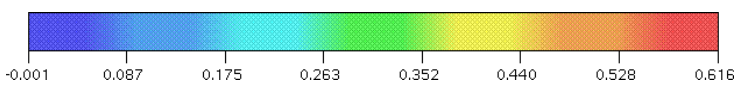

$0.05 \mathrm{~s}$

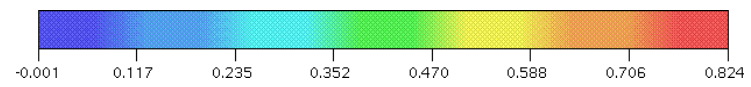

$0.10 \mathrm{~s}$
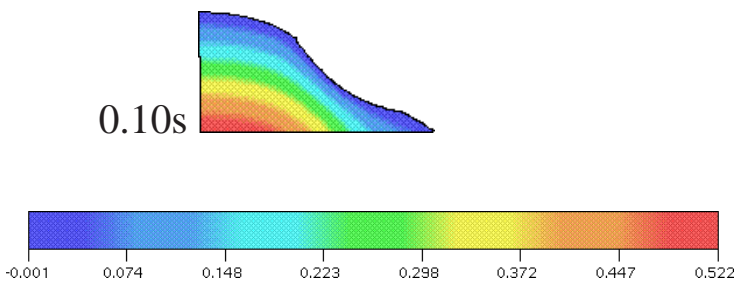

$0.15 \mathrm{~s}$
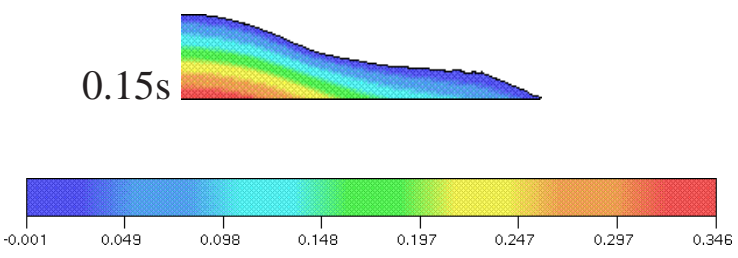

$0.20 \mathrm{~s}$

Figura 6.10: Distribuição do campo de pressão obtida na simulação do problema do colapso de coluna de fluido 2D em regime turbulento usando os esquemas ALUS e TOPUS. 
(a) Simulação direta

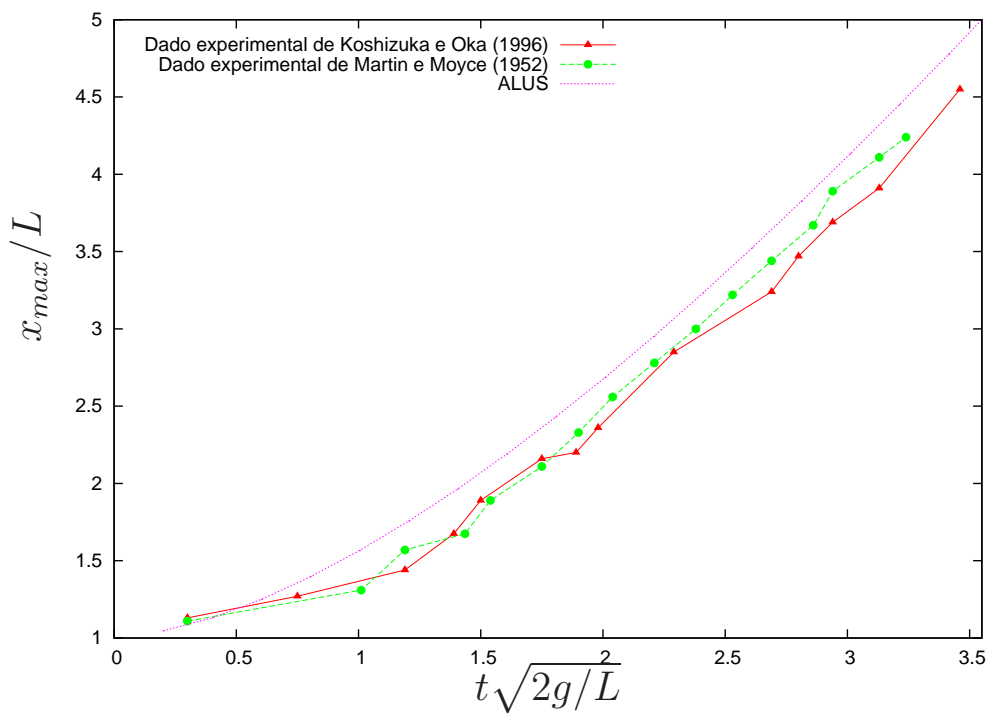

(b) Modelo $\kappa-\varepsilon$

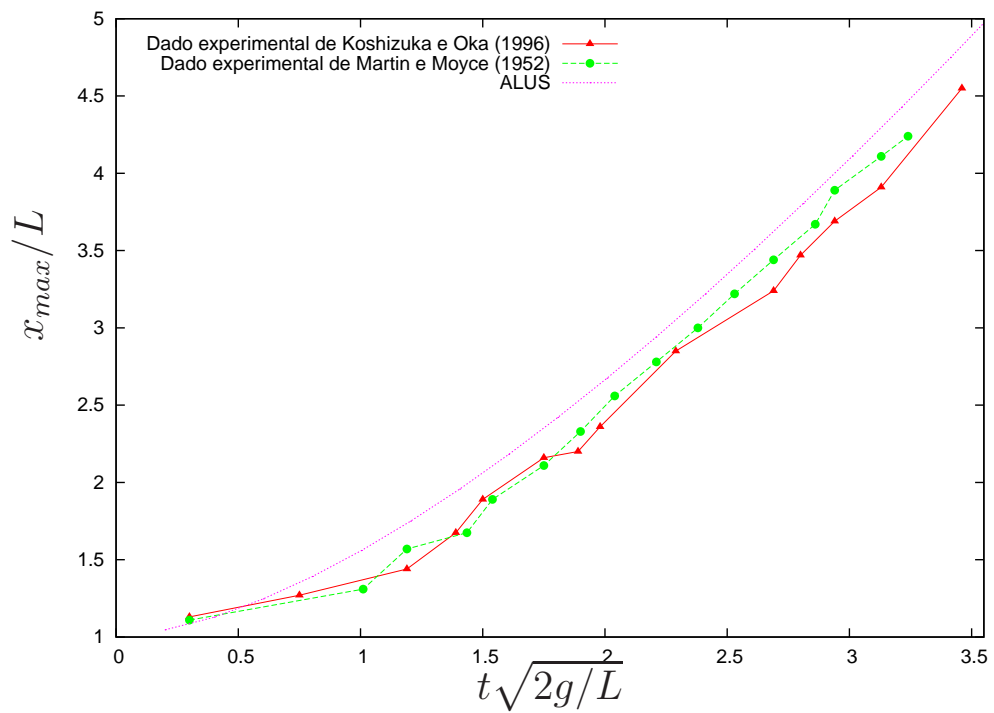

(c) Modelo RSAEM

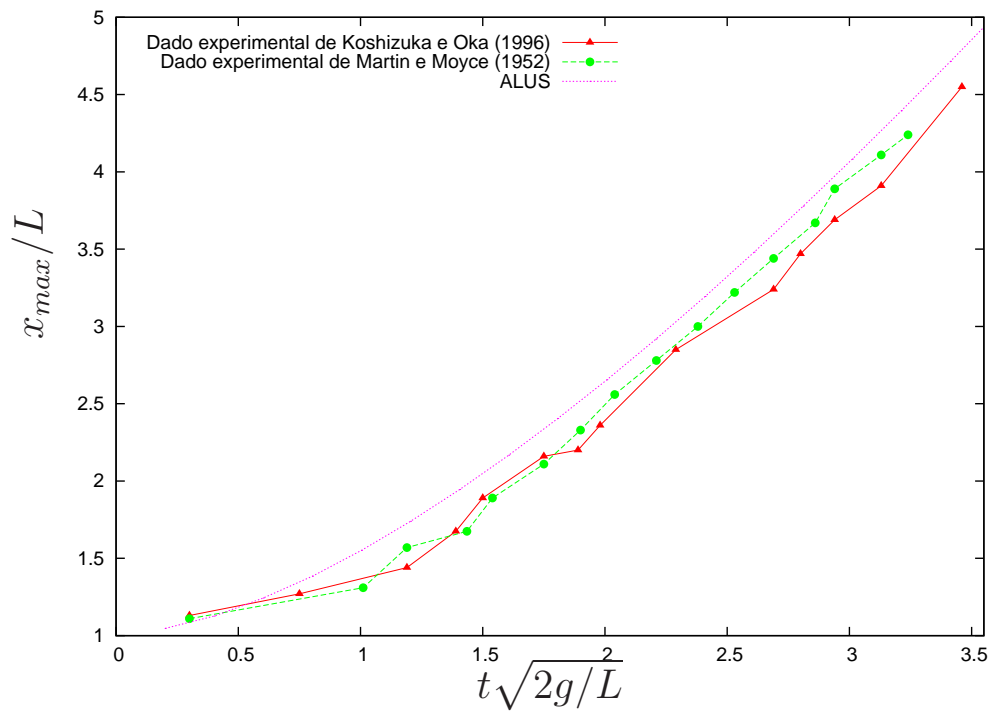

Figura 6.11: Comparação entre os dados experimentais e os resultados numéricos obtidos nas simulações usando o esquema ALUS com e sem modelagem de turbulência. 
(a) Simulação direta

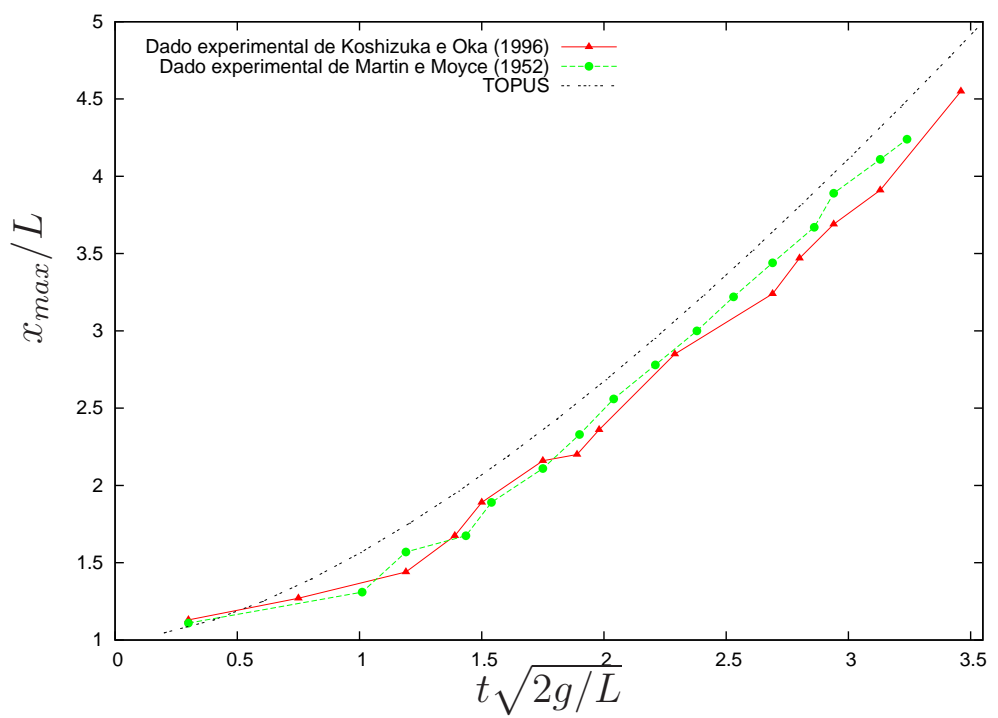

(b) Modelo $\kappa-\varepsilon$

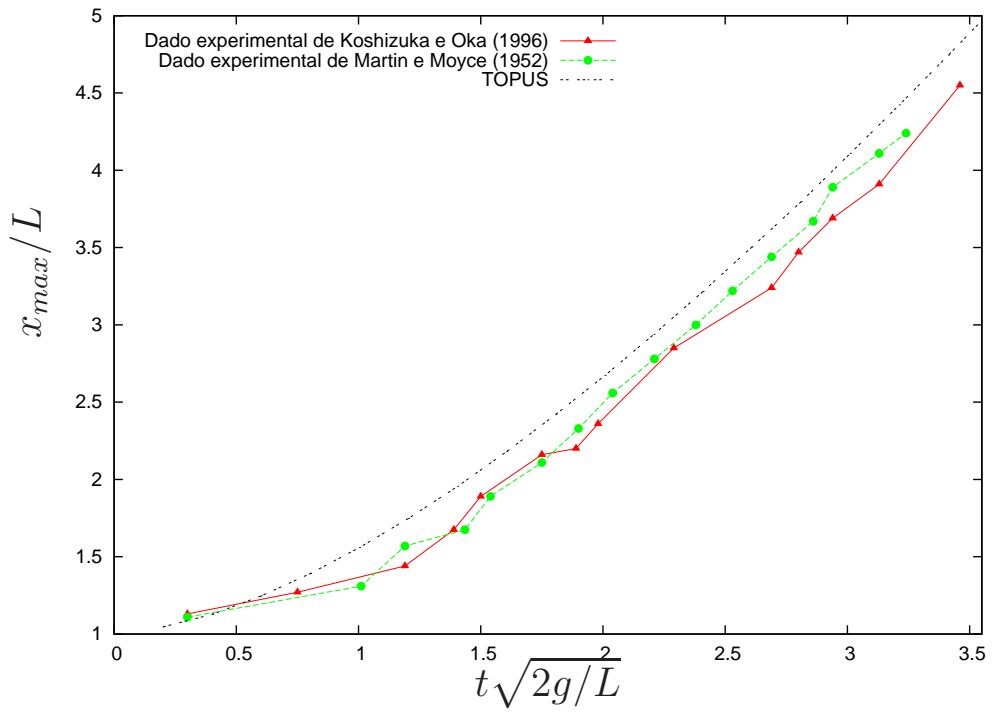

(c) Modelo RSAEM

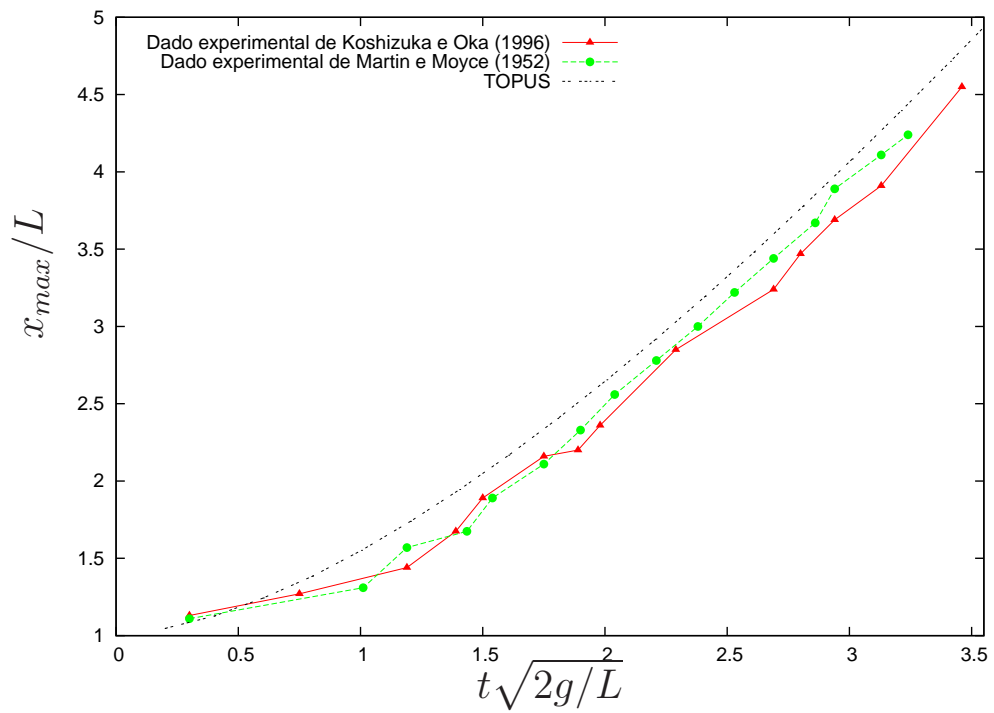

Figura 6.12: Comparação entre os dados experimentais e os resultados numéricos obtidos nas simulações usando o esquema TOPUS com e sem modelagem de turbulência. 


\subsubsection{Jato Livre sobre uma Superfície Rígida Impermeável}

Nesta seção são apresentadas simulações de escoamentos nos regimes laminar e turbulento de um jato livre incidindo perpendicularmente numa superfície rígida impermeável sob o efeito do campo gravitacional. Nessas simulações foram utilizados os esquemas ADBQUICKEST, ALUS, CUBISTA, TOPUS e VONOS. Os resultados mostrados nesta subseção foram parcialmente publicados em anais de eventos (ver $[112,113])$.

\section{Caso Laminar}

Aqui são apresentados os resultados numéricos no caso laminar. A geometria considerada para simular o problema está ilustrada na Figura 6.13. A solução analítica do Watson nesse caso é dada por (ver [155])

$$
h(x)= \begin{cases}\frac{\pi}{\sqrt{3}} \frac{\nu(x+l)}{Q}, & \text { para } x \geq x_{0}, \\ a+\frac{\left(3 \sqrt{3} c^{2}-2 \pi\right)}{3 \sqrt{3} c^{2}} \delta(x), & \text { para } x<x_{0},\end{cases}
$$

em que $h(x)$ é a altura da superfície livre, $U_{0}$ é a velocidade de injeção do fluido, $Q$ é vazão do fluido, $a=Q / U_{0}, c=1.402$. Os termos $\delta(x), x_{0}$ e $l$ são, respectivamente, definidos por:

$$
\begin{aligned}
\delta(x) & =\sqrt{\frac{3 \sqrt{3} c^{3}}{2(\pi-c \sqrt{3})} \frac{\nu x}{U_{0}}}, \\
x_{0} & =\left(\frac{3 c \sqrt{3}(\pi-c \sqrt{3})}{2 \pi^{2}}\right) a R e, \\
l & =\left(\frac{3 c \sqrt{3}(2 c \sqrt{3}-\pi)}{2 \pi^{2}}\right) a R e .
\end{aligned}
$$

Os dados do problema simulado, cujo domínio tem dimensões $0.4 m \times 0.04 m$, são o diâmetro do injetor $L=0.01 \mathrm{~m}$ (escala de comprimento ), a velocidade de injeção $U_{0}=1 \mathrm{~ms}^{-1}$, a constante gravitacional $g=9.81 \mathrm{~ms}^{-2}$ e a altura do injetor a partir da superfície rígida $H=0.037 \mathrm{~m}$. Nas simulações foram empregadas três malhas de $200 \times 20(\delta x=\delta y=0.002 m), 400 \times 40(\delta x=\delta y=$ $0.001 \mathrm{~m})$ e $800 \times 80(\delta x=\delta y=0.0005 \mathrm{~m})$ células computacionais e Reynolds $R e=2 \times 10^{3}$.

A Figura 6.15 apresenta as comparações entre as soluções numéricas obtidas com os esquemas ADBQUICKEST, ALUS, CUBISTA, TOPUS e a solução analítica de Watson [155]. A partir dessas figuras, vê-se que as soluções numéricas convergiram para a solução analítica com o refinamento da malha. As simulações com o esquema TOPUS nas malhas de $200 \times 20$ e $400 \times 40$ células produziram soluções numéricas oscilatórias, a qual foi estabilizada quando se empregou a malha mais fina. Em contrapartida, o esquema ALUS não apresentou vestígios de instabilidades. Como ilustração, a Figura 6.14 apresenta o campo de pressão no tempo $t=2 s$. 


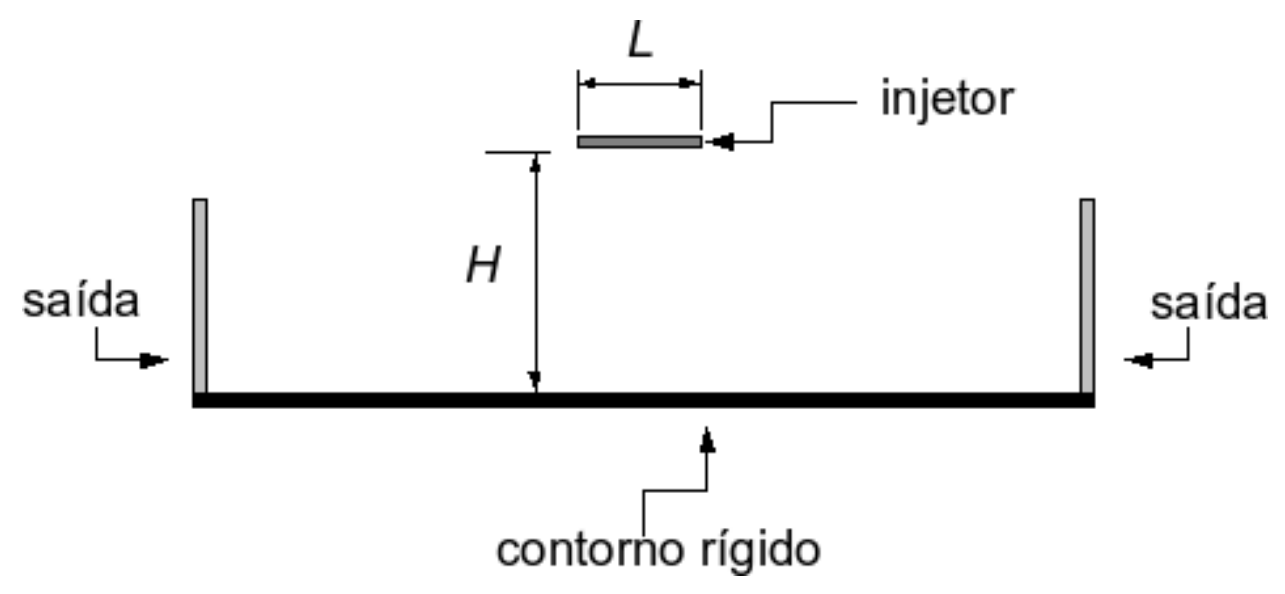

Figura 6.13: Geometria de um jato livre incidindo sobre um contorno rígido.

(a) ADBQUICKEST

(b) ALUS

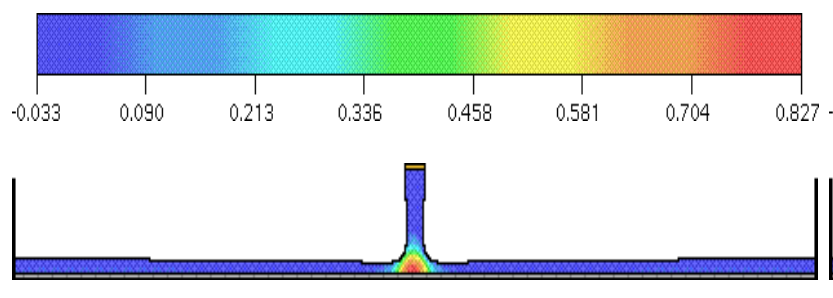

(c) CUBISTA

(d) TOPUS
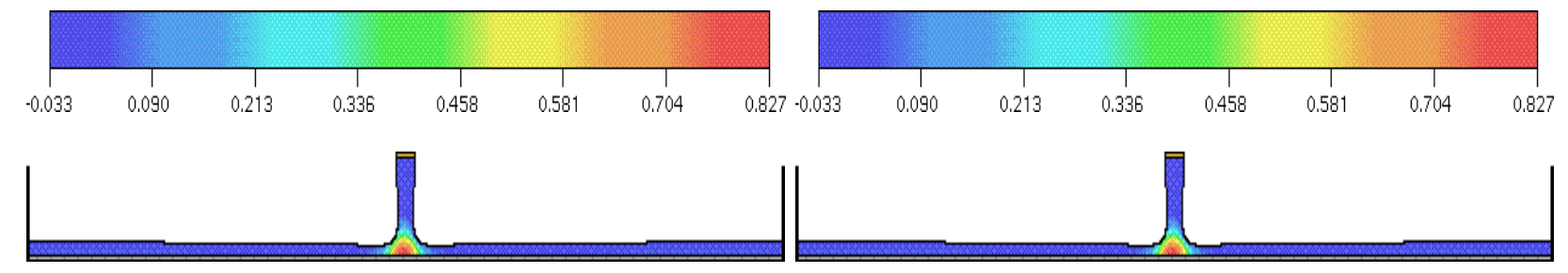

Figura 6.14: Resultados numéricos para o campo de pressão do escoamento em regime laminar de um jato livre incidindo sobre um contorno rígido. 
(a) ADBQUICKEST

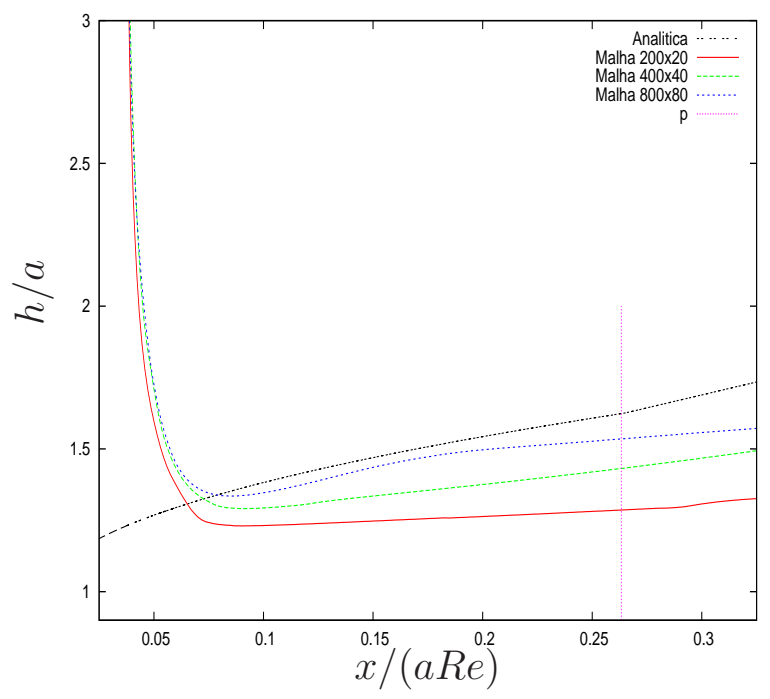

(c) CUBISTA

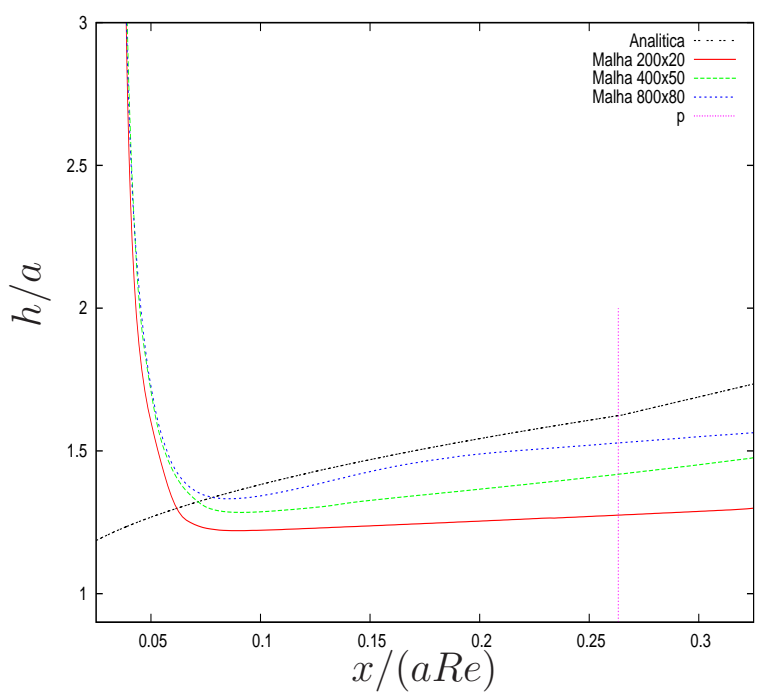

(b) ALUS

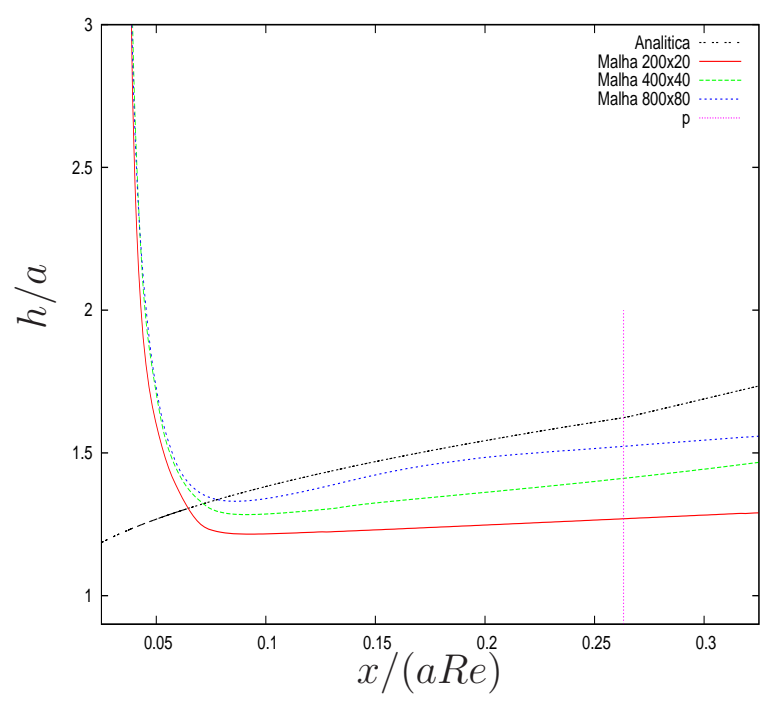

(d) TOPUS

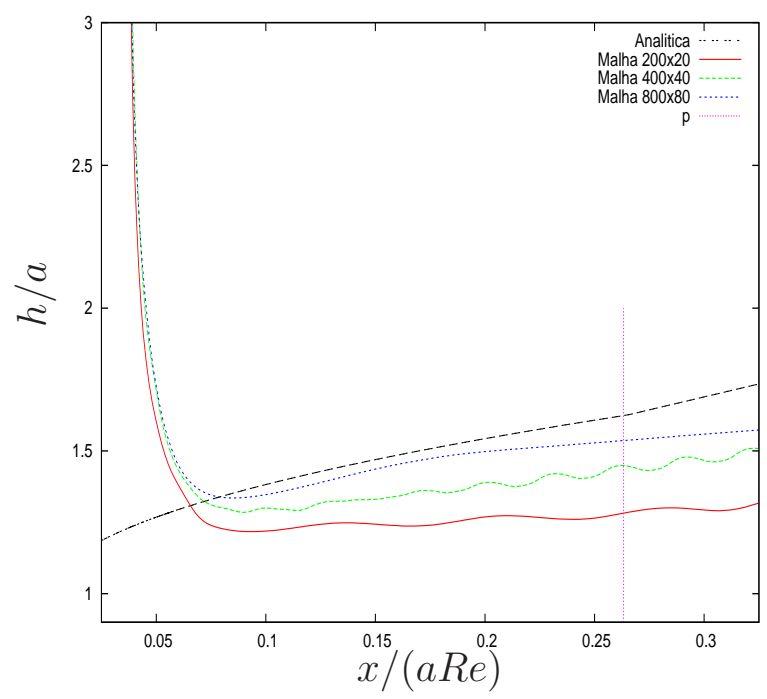

Figura 6.15: Comparação entre a solução analítica de Watson e as soluções numéricas obtidas com os esquemas ADBQUICKEST, ALUS, CUBISTA e TOPUS no caso laminar, $\mathrm{p}=x_{0} /(a R e)$. 


\section{Caso Turbulento}

Neste caso, Watson [155] também apresenta uma solução analítica, a qual foi utilizada para verificar os esquemas "upwind" abordados nesta subseção. A solução de Watson nesse caso é dada por

$$
h(x)= \begin{cases}\frac{81(7 A)^{\frac{1}{4}} k}{800}\left(\frac{\nu}{Q}\right)^{\frac{1}{4}}(x+l), & x \geq x_{0}, \\ a+\left(1-\frac{A}{k}\right) \delta(x), & x<x_{0},\end{cases}
$$

em que $A=0.239$ e $k=0.260$. Em (6.4), os parâmetros $\delta(x), x_{0}$ e $l$ são calculados por

$$
\begin{aligned}
\delta(x) & =\left(\frac{81}{320(9 A-2)}\right)^{\frac{4}{5}} 7^{\frac{1}{5}} k\left(\frac{a \nu}{Q}\right)^{\frac{1}{5}} x^{\frac{4}{5}}, \\
x_{0} & =\frac{320(9 A-2)}{81 \times 7^{\frac{1}{4}} A^{\frac{5}{4}}} a R e^{\frac{1}{4}}, \\
l & =\frac{160(1-2 A)}{9 \times 7^{\frac{1}{4}} A^{\frac{5}{4}}} a R e^{\frac{1}{4}} .
\end{aligned}
$$

Os dados do problema simulado, cujo domínio tem dimensões $0.2 m \times 0.05 m$, são o diâmetro do injetor $L=0.01 \mathrm{~m}$, a escala de velocidade $U_{0}=1 \mathrm{~ms}^{-1}$ (a velocidade de injeção), a escala de comprimento $L$, a constante gravitacional $g=9.81 \mathrm{~ms}^{-2}$, e a altura do injetor a partir da superfície rígida $H=0.015 \mathrm{~m}$. Nas simulações foram empregadas as malhas $200 \times 50(\delta x=\delta y=0.001 \mathrm{~m})$, $400 \times 100(\delta x=\delta y=0.0005 m)$ e $800 \times 200(\delta x=\delta y=0.00025 m)$ e Reynolds $R e=5 \times 10^{4}$.

As Figuras 6.16 e 6.17 mostram as comparações das soluções numéricas, obtidas com os modelos $\kappa-\varepsilon$ e RSAEM, e analítica de Watson. Pode-se perceber dessas figuras que, de maneira geral, as soluções numéricas nas malhas grossa e intermediária convergiram para a solução na malha mais fina. Observa-se também que as simulações com a modelagem $\kappa-\varepsilon$ (Figura 6.16) não apresentaram oscilações, ao passo que as simulações com a modelagem RSAEM (Figura 6.17) apresentaram problemas, isto é, essa modelagem associada com os esquemas ALUS e CUBISTA mostrou-se inadequada. Em suma, pode-se concluir que, nesse problema com superfície livre, a modelagem $\kappa-\varepsilon$ mostrou ser superior. E isso é na opinião do autor uma surpresa, uma vez que o modelo matemático do RSAEM é mais rigoroso e propõe eliminar algumas deficiências da modelagem clássica. 
(a) ADBQUICKEST

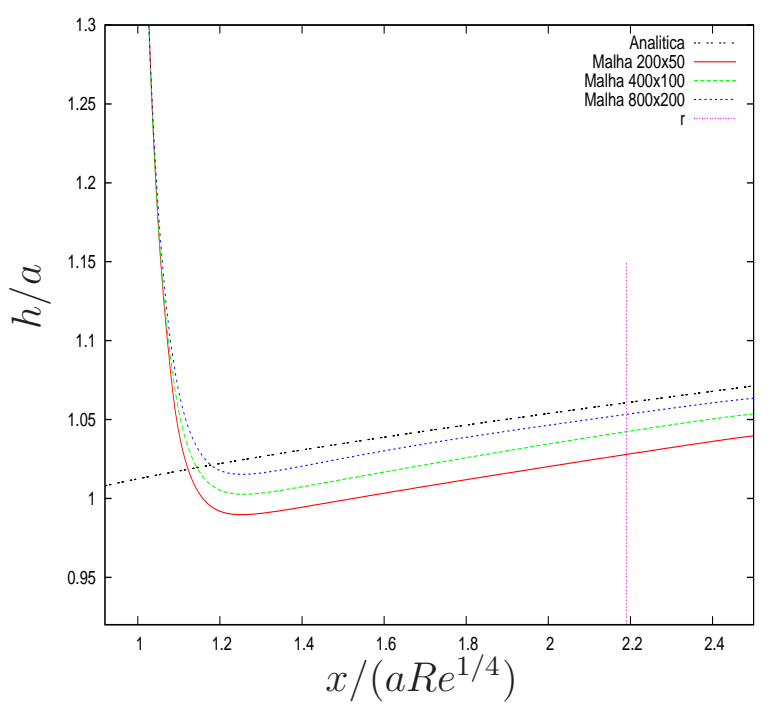

(c) CUBISTA

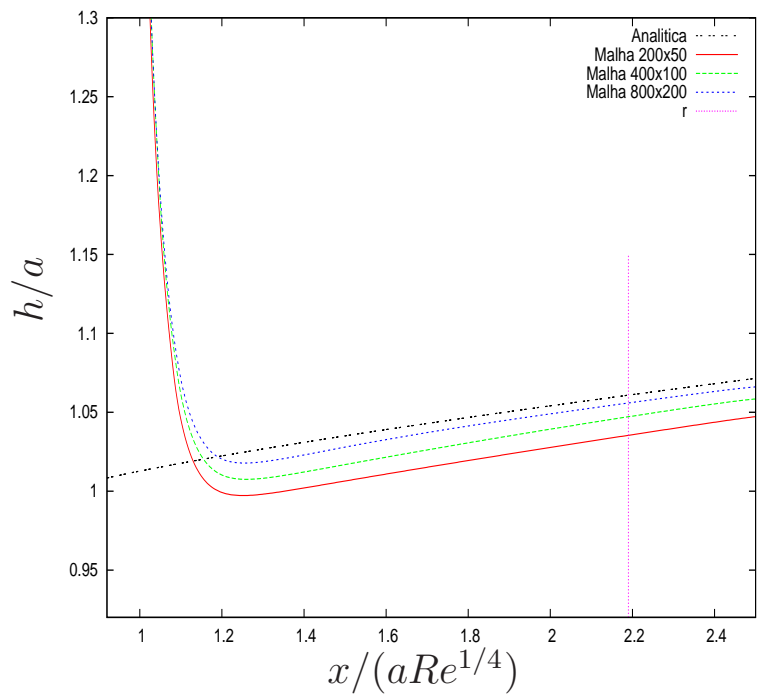

(b) ALUS

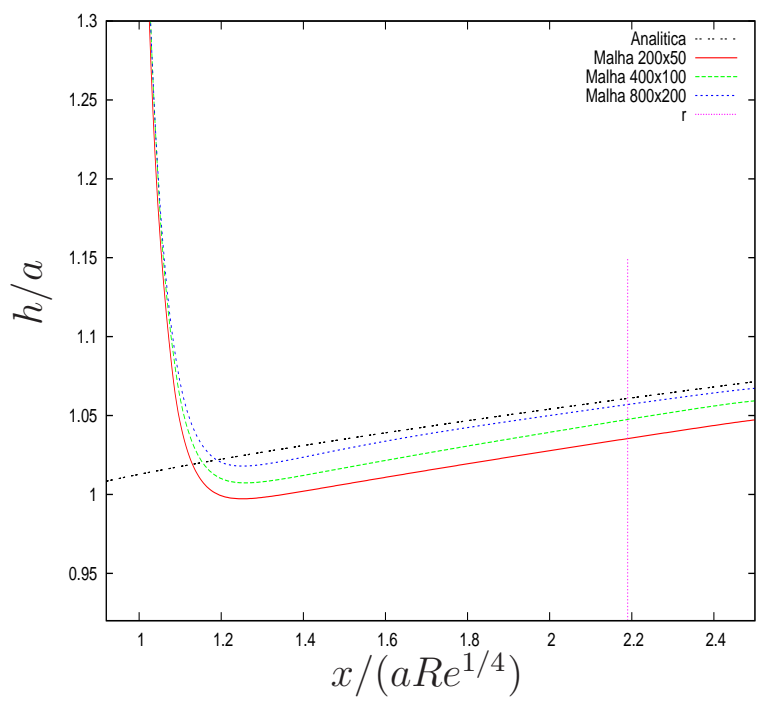

(d) TOPUS

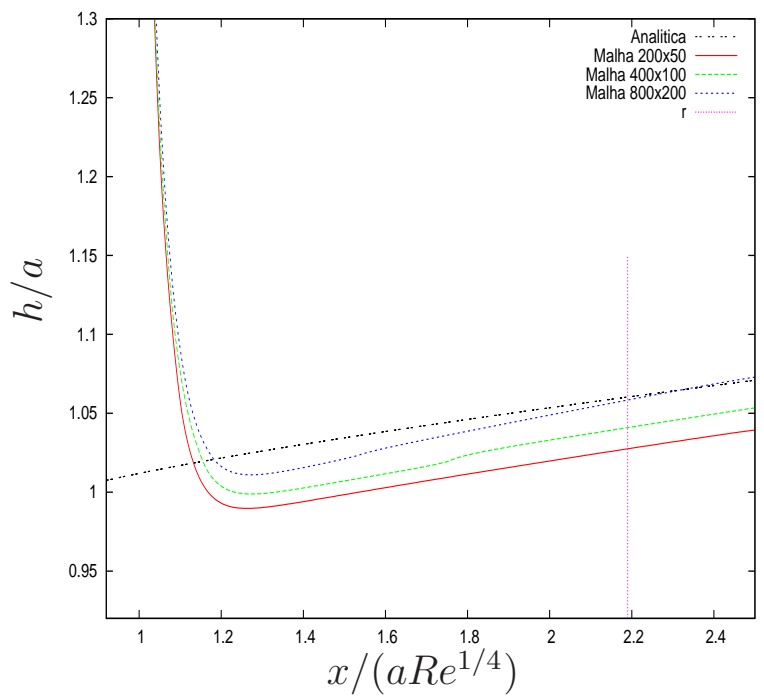

Figura 6.16: Comparação entre a solução analítica de Watson e os resultados numéricos obtidos com os esquemas ADBQUICKEST, ALUS, CUBISTA e TOPUS combinados com o modelo $\kappa-\varepsilon$, $\mathrm{r}=x_{0} /\left(a R e^{1 / 4}\right)$. 
(a) ADBQUICKEST

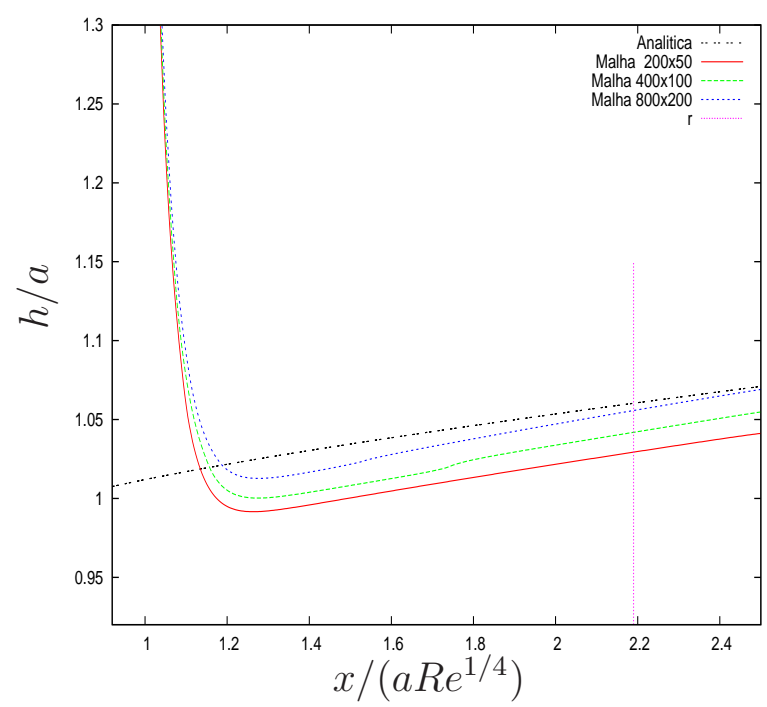

(c) CUBISTA

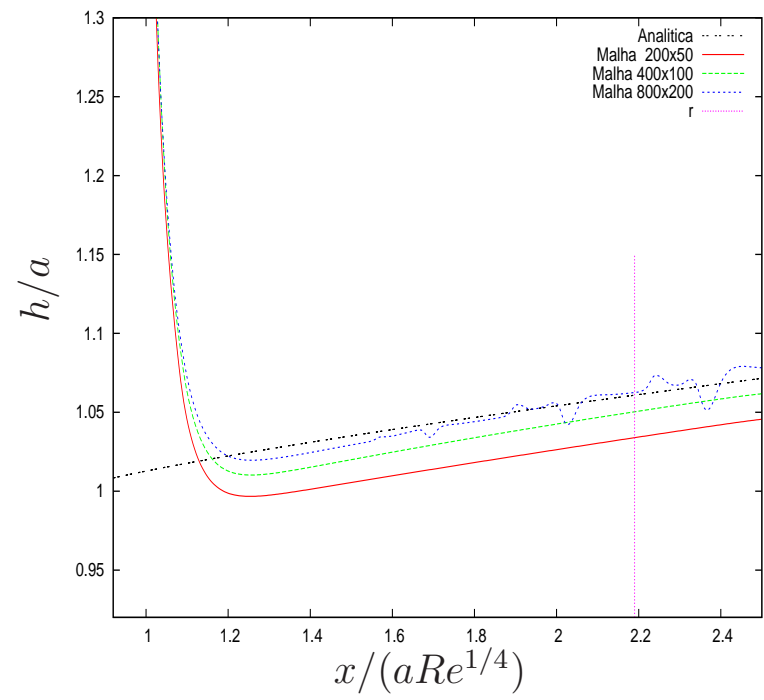

(b) ALUS

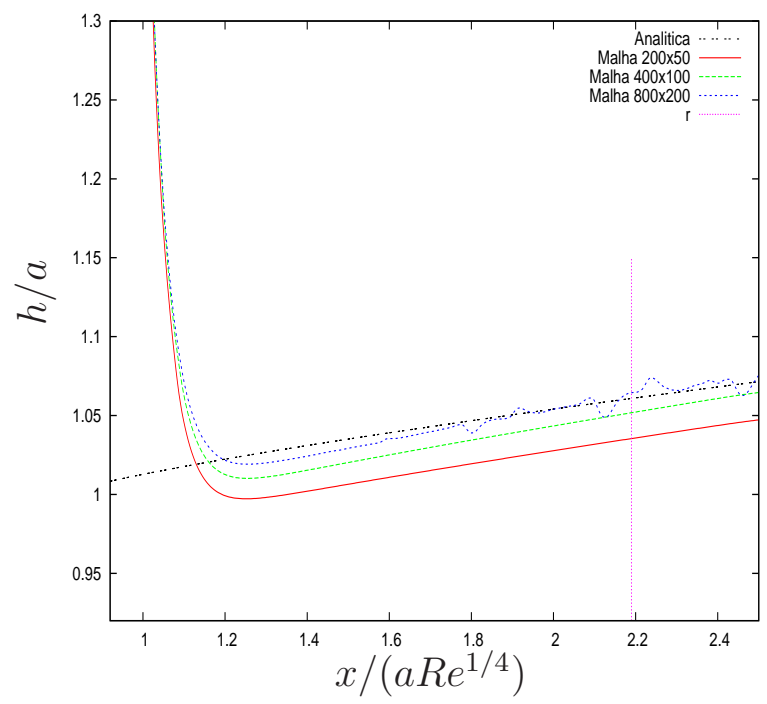

(d) TOPUS

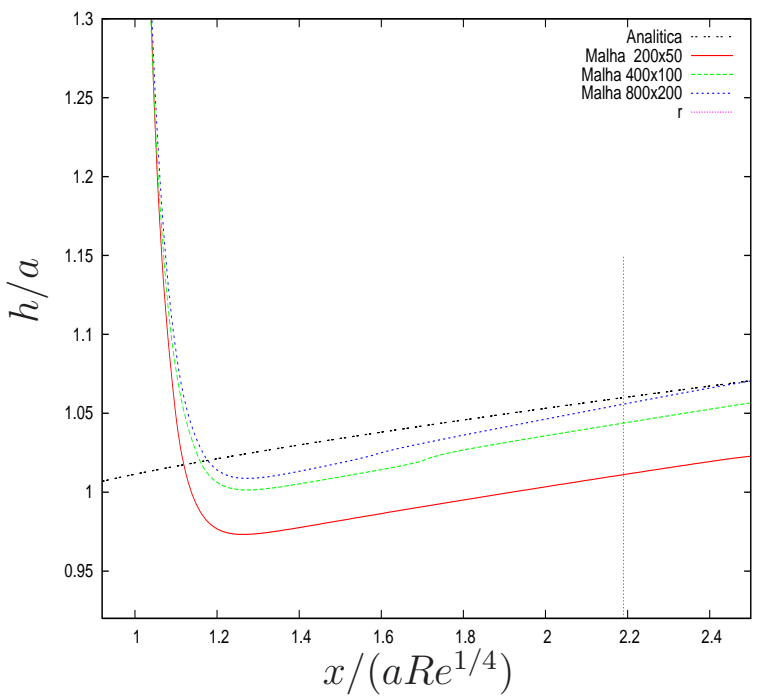

Figura 6.17: Comparação entre a solução analítica de Watson e os resultados numéricos obtidos com os esquemas ADBQUICKEST, ALUS, CUBISTA e TOPUS combinados com o modelo $\mathrm{RSAEM}, \mathrm{r}=x_{0} /\left(a R e^{1 / 4}\right)$. 


\subsubsection{Aplicação: Escoamentos de um Fluido Viscoelástico}

Nesta seção são apresentados os resultados numéricos obtidos na simulação de escoamentos viscoelásticos com/sem superfícies livres de um fluido Oldroyd-B [58, 88]. Nessas simulações, os esquemas ALUS e TOPUS foram usados para estimar os termos convectivos das EDPs (2.55) e (2.56).

\subsubsection{Escoamento num Canal}

Neste problema é considerado um canal de dimensões $5 m \times 1 m$ vazio. Inicialmente, o fluido é injetado na seção de entrada e, após um determinado tempo, ele alcança a região de saída. As soluções analíticas do problema em estado estacionário são dadas por [15]

$$
\begin{gathered}
u(y)=-6 y(y-L), \quad v(y)=0 \\
T_{11}(y)=2 W e(1-\beta)\left(\frac{\partial u}{\partial y}\right)^{2}, \quad T_{12}(y)=(1-\beta)\left(\frac{\partial u}{\partial y}\right), \quad T_{22}(y)=0 .
\end{gathered}
$$

Para a simulação deste problema, os seguintes dados foram utilizados: escala de comprimento $L=1.0 \mathrm{~m}$, velocidade de injeção $U_{0}=1 \mathrm{~ms}^{-1}$, tempo de relaxação $\lambda_{1}=2.0 \mathrm{~s}$, tempo de retardamento $\lambda_{2}=1.0 \mathrm{~s}$, número de Weissenberg $W e=2.0$ e números de Reynolds $R e=0.01$ e $R e=0.1$. Quatro malhas foram adotadas para a simulação: $M 1=25 \times 5, M 2=50 \times 10$, $M 3=100 \times 20$ e $M 4=200 \times 40$ (células computacionais). Os resultados apresentados na seqüência são obtidos com o uso dos esquemas ALUS e TOPUS.

A Figura 6.18 mostra os contornos obtidos com o TOPUS no tempo $t=2.175 \mathrm{~s}$ para a velocidade $u$, a pressão $p$ e os tensores $T_{11}$ e $T_{12}$. Os perfis estacionários para $u, T_{11}$ e $T_{12}$ na metade do canal $(x=2.5 m)$ e no tempo $t=100 s$ são apresentados na Figura 6.19. Esses perfis obtidos com o esquema ALUS mostram ser qualitativamente semelhantes aos obtidos com o esquema TOPUS e, por essa razão, foram omitidos.

Com o objetivo de comparar os esquemas ALUS e TOPUS nesse problema, os erros $\|E\|_{2}$ entre as soluções numéricas e analíticas (ver [15]) em cada malha foram calculados. Esses dados mais as estimativas para a ordem numérica estão mostrados nas Tabelas 6.5 e 6.6, respectivamente. Nota-se que ambos os esquemas apresentaram resultados similares e atingiram, no global, ordem de convergência próximo de 2. 
(a) $u$

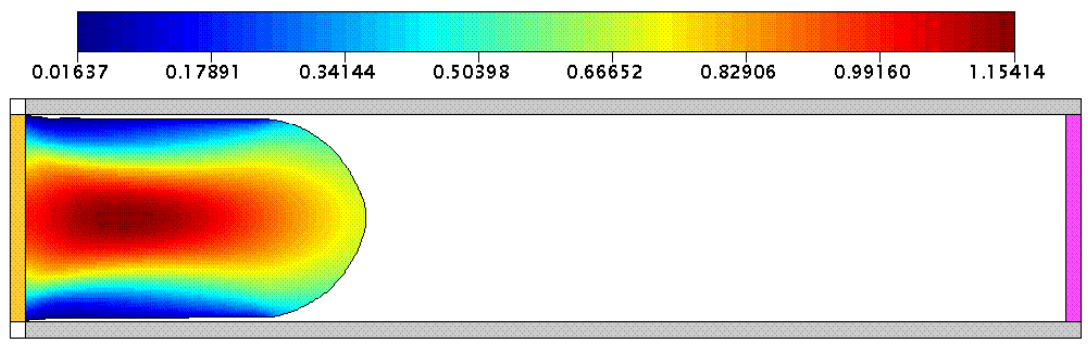

(b) $p$

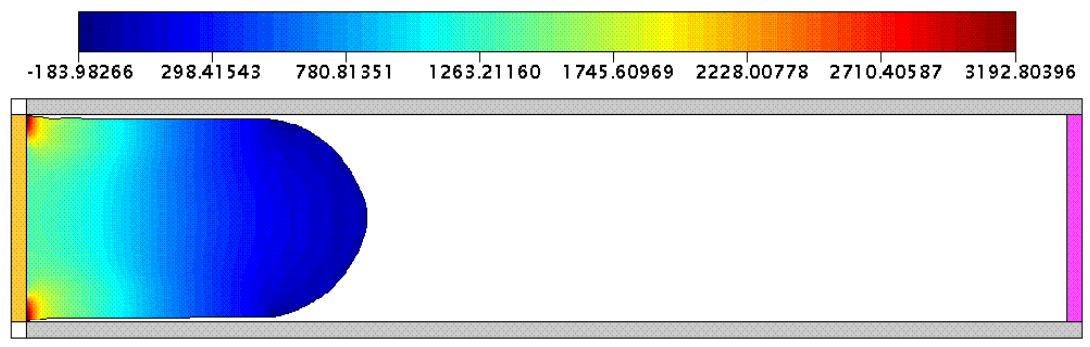

(c) $T_{11}$

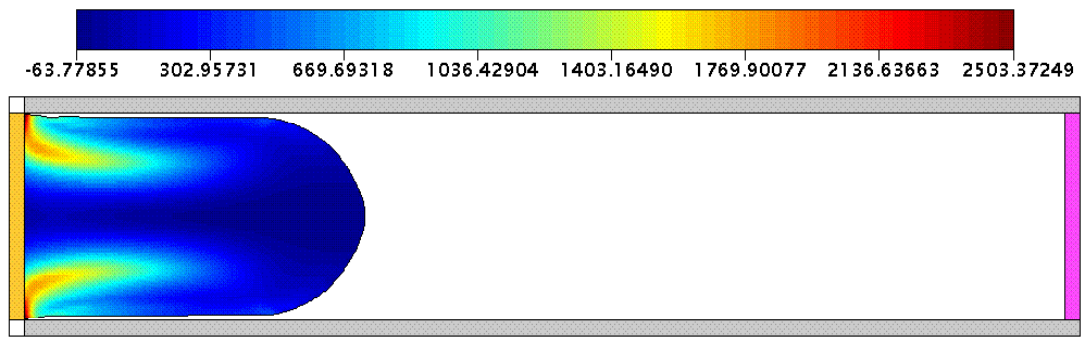

(d) $T_{12}$

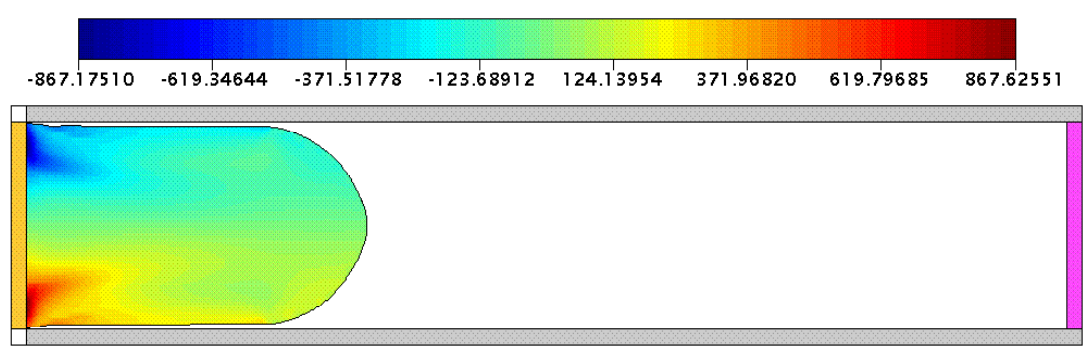

Figura 6.18: Soluções numéricas obtidas na simulação com o esquema TOPUS no instante $t=$ 2.175 s na malha de $200 \times 40$ células a Reynolds $R e=0.01$. 
(a) Soluções numéricas e exata da componente $u$ da velocidade.

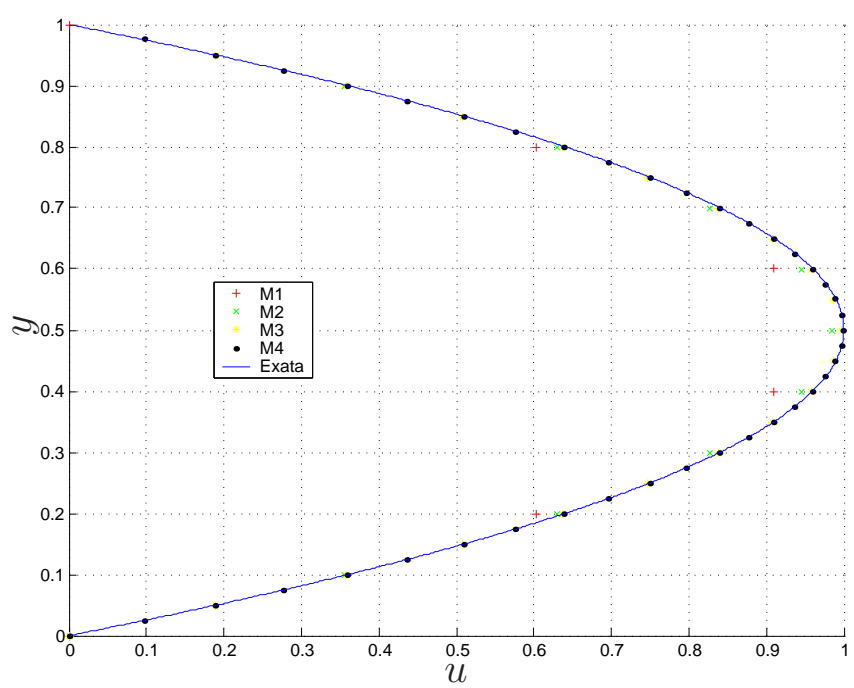

(b) Soluções numéricas e exata da componente $T_{11}$ do tensor de tensão.

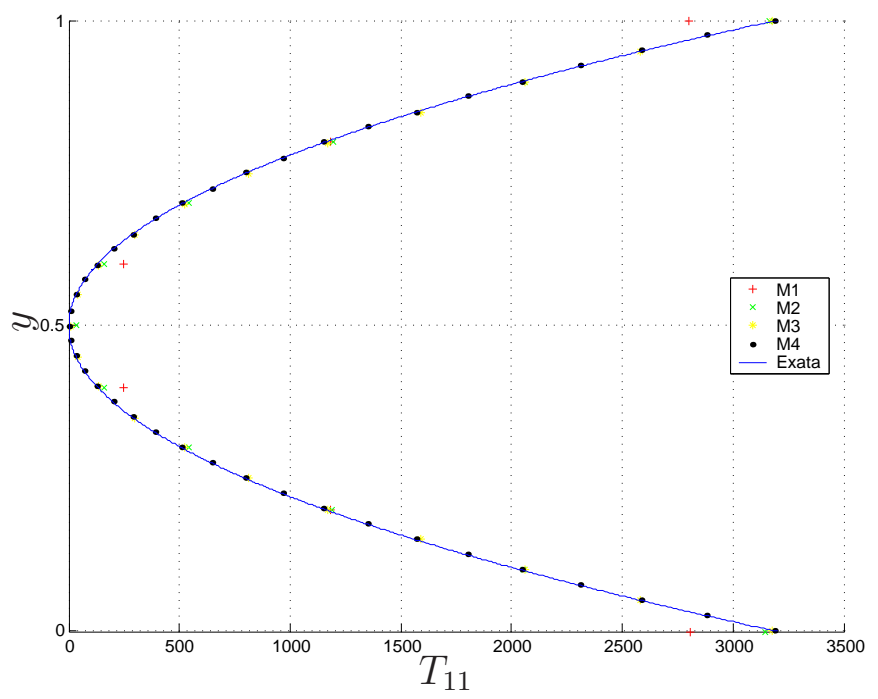

(c) Soluções numéricas e exata da componente $T_{12}$ do tensor de tensão.

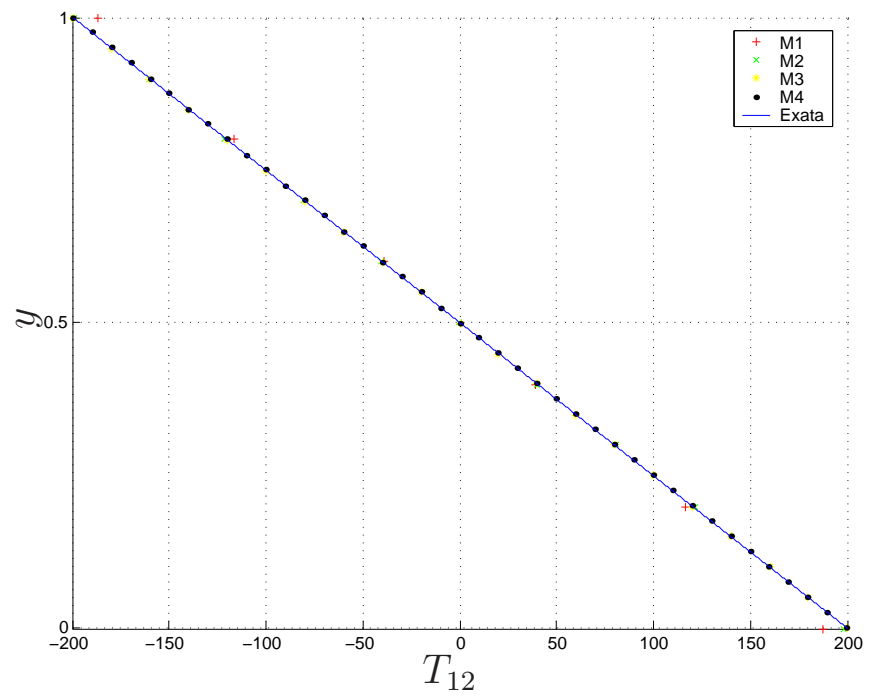

Figura 6.19: Comparação entre as soluções numéricas, obtidas com a utilização do esquema TOPUS a Reynolds $R e=0.01$ em diferentes malhas, e analíticas. 
Tabela 6.5: Escoamento viscoelástico num canal - Erro $\|E\|_{2}$ entre as soluções numérica e analítica.

\begin{tabular}{cccccc}
\hline \hline & & & \multicolumn{3}{c}{ Erro $\|E\|_{2}$} \\
\cline { 3 - 5 } Esquema & $\operatorname{Re}$ & Malha & $u$ & $T_{11}$ & $T_{12}$ \\
\hline & & $25 \times 5$ & $5.4210 \times 10^{-2}$ & $1.2187 \times 10^{-1}$ & $5.6288 \times 10^{-2}$ \\
& $50 \times 10$ & $1.4995 \times 10^{-2}$ & $1.9986 \times 10^{-2}$ & $6.8054 \times 10^{-3}$ \\
& 0.01 & $100 \times 20$ & $3.5969 \times 10^{-3}$ & $8.0583 \times 10^{-3}$ & $2.7897 \times 10^{-3}$ \\
& & $200 \times 40$ & $9.1137 \times 10^{-4}$ & $2.1099 \times 10^{-3}$ & $1.4114 \times 10^{-3}$
\end{tabular}

ALUS

\begin{tabular}{|c|c|c|c|c|c|}
\hline & \multirow{4}{*}{0.1} & $25 \times 5$ & $5.4213 \times 10^{-2}$ & $1.2184 \times 10^{-1}$ & $5.6278 \times 10^{-2}$ \\
\hline & & $50 \times 10$ & $1.4995 \times 10^{-2}$ & $1.9979 \times 10^{-2}$ & $6.8117 \times 10^{-3}$ \\
\hline & & $100 \times 20$ & $3.5968 \times 10^{-3}$ & $8.0649 \times 10^{-3}$ & $2.7921 \times 10^{-3}$ \\
\hline & & $200 \times 40$ & $9.1133 \times 10^{-4}$ & $2.1118 \times 10^{-3}$ & $1.4113 \times 10^{-3}$ \\
\hline \multirow{9}{*}{ TOPUS } & \multirow{5}{*}{0.01} & $25 \times 5$ & $5.4168 \times 10^{-2}$ & $1.2234 \times 10^{-1}$ & $5.6382 \times 10^{-2}$ \\
\hline & & $50 \times 10$ & $1.5037 \times 10^{-2}$ & $1.9567 \times 10^{-2}$ & $6.8238 \times 10^{-3}$ \\
\hline & & $100 \times 20$ & $3.5895 \times 10^{-3}$ & $8.3832 \times 10^{-3}$ & $2.8868 \times 10^{-3}$ \\
\hline & & $200 \times 40$ & $9.1447 \times 10^{-4}$ & $2.0753 \times 10^{-3}$ & $1.4258 \times 10^{-3}$ \\
\hline & & & & & \\
\hline & \multirow{4}{*}{0.1} & 25 & $\times 10^{-2}$ & $\times 10^{-1}$ & $<10^{-2}$ \\
\hline & & & $1.5037 \times 10^{-2}$ & $1.9561 \times 10^{-2}$ & $6.8282 \times 10^{-}$ \\
\hline & & $100 \times 20$ & $3.5894 \times 10^{-3}$ & $8.3899 \times 10^{-3}$ & $2.8891 \times 10^{-5}$ \\
\hline & & $200 \times 40$ & $9.1443 \times 10^{-4}$ & $2.0768 \times 10^{-3}$ & $1.4260 \times 10^{-3}$ \\
\hline
\end{tabular}


Tabela 6.6: Escoamento viscoelástico num canal - Taxa da convergência do método numérico na norma 2.

\begin{tabular}{cccccc}
\hline \hline \multirow{2}{*}{ Esquema } & & & \multicolumn{3}{c}{ Taxa de convergência $-\|E\|_{2}$} \\
\cline { 3 - 6 } & & Malha & $u$ & $T_{11}$ & $T_{12}$ \\
\hline & & $50 \times 5$ & - & - & - \\
& & $100 \times 20$ & 2.0597 & 1.3104 & 1.2866 \\
& 0.01 & $200 \times 40$ & 1.9806 & 1.9333 & 0.9829
\end{tabular}

ALUS

\begin{tabular}{ccccc} 
& $25 \times 5$ & - & - & - \\
& $50 \times 10$ & 1.8542 & 2.6084 & 3.0465 \\
& $100 \times 20$ & 2.0597 & 1.3088 & 1.2866 \\
0.1 & $200 \times 40$ & 1.9807 & 1.9332 & 0.9843 \\
\hline & $25 \times 5$ & - & - & - \\
& $50 \times 10$ & 1.8489 & 2.6444 & 3.0466 \\
& $100 \times 20$ & 2.0667 & 1.2228 & 1.2411 \\
0.01 & $200 \times 40$ & 1.9728 & 2.0141 & 1.0177
\end{tabular}

TOPUS

\begin{tabular}{cccc}
$25 \times 5$ & - & - & - \\
$50 \times 10$ & 1.8490 & 2.6445 & 3.0454 \\
$100 \times 20$ & 2.0667 & 1.2213 & 1.2409 \\
$200 \times 40$ & 1.9728 & 2.0143 & 1.0187 \\
\hline
\end{tabular}




\subsubsection{Jato Oscilante}

Nesta subseção são apresentados resultados numéricos obtidos na simulação de um jato oscilante. É importante informar ao leitor que soluções analíticas e experimentais não são disponíveis na literatura para uma comparação quantitativa com os dados numéricos descritos neste trabalho. O objetivo aqui é investigar o comportamento do método GENSMAC equipado com os esquemas ALUS e TOPUS quando aplicado na simulação de um problema de escoamento de fluido altamente viscoso. Pretende-se apenas mostrar que o método simula com sucesso o fenômeno de dobras (em inglês "buckling" - ver as referências [27, 28, 58, 69]).

Para a simulação deste problema envolvendo um jato viscoelástico, foram adotados os seguintes dados: escala de comprimento $L=3.0 \times 10^{-3} \mathrm{~m}$ (comprimento do injetor); constantes temporais $\lambda_{1}=1.5 \times 10^{-2} s, \lambda_{2}=3.0 \times 10^{-3} \mathrm{~s}$; escala de velocidade $U_{0}=0.5 \mathrm{~ms}^{-1}$; altura do injetor $H=0.047 \mathrm{~m}$; constante gravitacional $\mathrm{g}=9.81 \mathrm{~ms}^{-2}$. Os números de Reynolds e de Weissenberg das simulações foram, respectivamente, $R e=0.15$ e $W e=2.5$. Nas simulações, a malha utilizada possui $100 \times 75$ células $\left(\delta x=\delta y=5.0 \times 10^{-4} \mathrm{~m}\right)$. O espaçamento temporal e o tempo final de simulação são $\delta t=3.0 \times 10^{-5} s$ e $t=0.78 \mathrm{~s}$, respectivamente.

Na Figura 6.20 (e sua continuação 6.21) são mostrados os resultados numéricos obtidos em diferentes tempos de simulação para o campo de velocidade na direção vertical (eixo $y$ ) utilizando o método GENSMAC combinado com o esquema TOPUS. É importante informar ao leitor que soluções analíticas e experimentais não são disponíveis na literatura para uma comparação quantitativa com os dados numéricos descritos neste trabalho. Porém, vale salientar que esses dados numéricos estão em boa concordância com os resultados de Grossi [58] e de Kim et al. [69]. Além disso, estes resultados são consistentes dentro da metodologia de simulação de escoamentos viscoelásticos apresentada por Martins [88]. Os resultados obtidos com o ALUS foram qualitativamente semelhantes aos obtidos com o TOPUS e, por isso, eles foram omitidos. 
(a) $t=0.081 \mathrm{~s}$

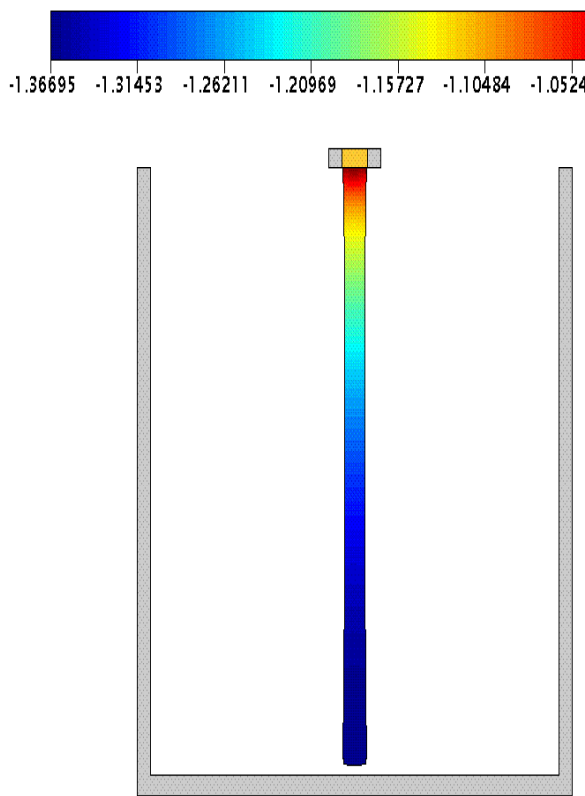

(b) $t=0.09 \mathrm{~s}$

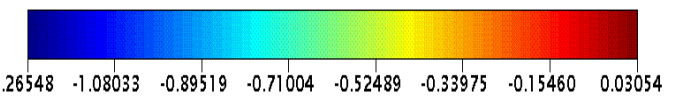

Figura 6.20: Resultados numéricos da componente $v$ da velocidade obtidos usando o esquema TOPUS a Reynolds $R e=0.15$ e a Weissenberg $W e=2.5$.

\subsubsection{Aplicação: Problema "Sluice Gate"}

Nesta subseção são apresentados resultados numéricos para o problema "sluice gate" (ver $[60,93])$. Os resultados obtidos na simulação deste problema foram parcialmente publicados em anais de eventos e periódico (ver $[48,112])$. O problema é geralmente usado como um modelo para estudar escoamentos em estruturas hidráulicas [93, 122], onde se determinam os efeitos da superfície livre e a distribuição de pressão. Esse problema é parecido com o do colapso de um fluido descrito na subseção 6.1.3, porém com um violento movimento da superfície livre gerado pelo "sluice gate".

Os dados para a simulação do problema são os mesmos daqueles utilizados no problema do colapso de uma coluna de fluido (ver subseção 6.1.3). As soluções numéricas para o campo de pressão e componentes da velocidade obtidas sem modelagem de turbulência com os esquemas ALUS e TOPUS estão apresentadas, respectivamente, nas Figuras 6.23, 6.24 e 6.25. Dessas figuras vê-se claramente a formação das estruturas turbilhonares (grandes vórtices) e suas interações com a superfície livre.

\subsubsection{Aplicação: Jato num Corpo de Fluido em Repouso}

Nesta subseção são apresentados os resultados numéricos da simulação de um jato penetrando num corpo de fluido em repouso a uma distância $H=0.6 \mathrm{~m}$ da superfície livre (ver Brandi [17]). Os resultados apresentados nesta subseção foram parcialmente publicados em anais de eventos (ver [112]). A geometria do modelo simulado está ilustrada na Figura 6.22. Nesta aplicação os seguintes dados foram adotados: $D=0.05 \mathrm{~m}$; velocidade do fluido no injetor $U=2 \mathrm{~ms}^{-1}$; Reynolds $R e=5 \times 10^{4}$; e malha de $100 \times 100$ células computacionais $(\delta x=\delta y=0.01 \mathrm{~m})$. Os esquemas ALUS e TOPUS são usados nesta aplicação. 
(c) $t=0.24 \mathrm{~s}$

(d) $t=0.30 \mathrm{~s}$

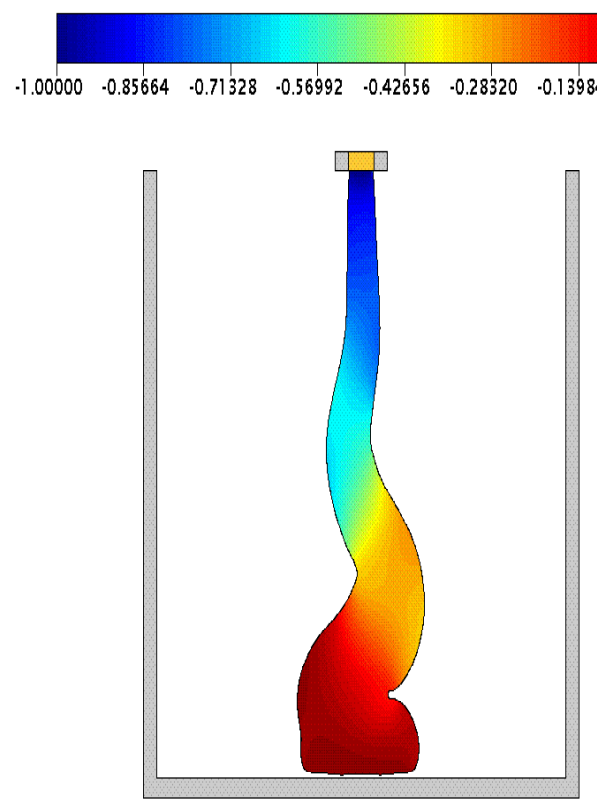

(e) $t=0.60 \mathrm{~s}$

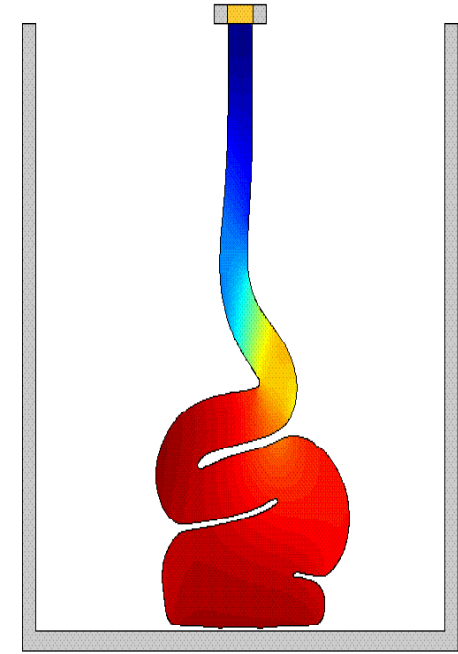

(f) $t=0.78 \mathrm{~s}$
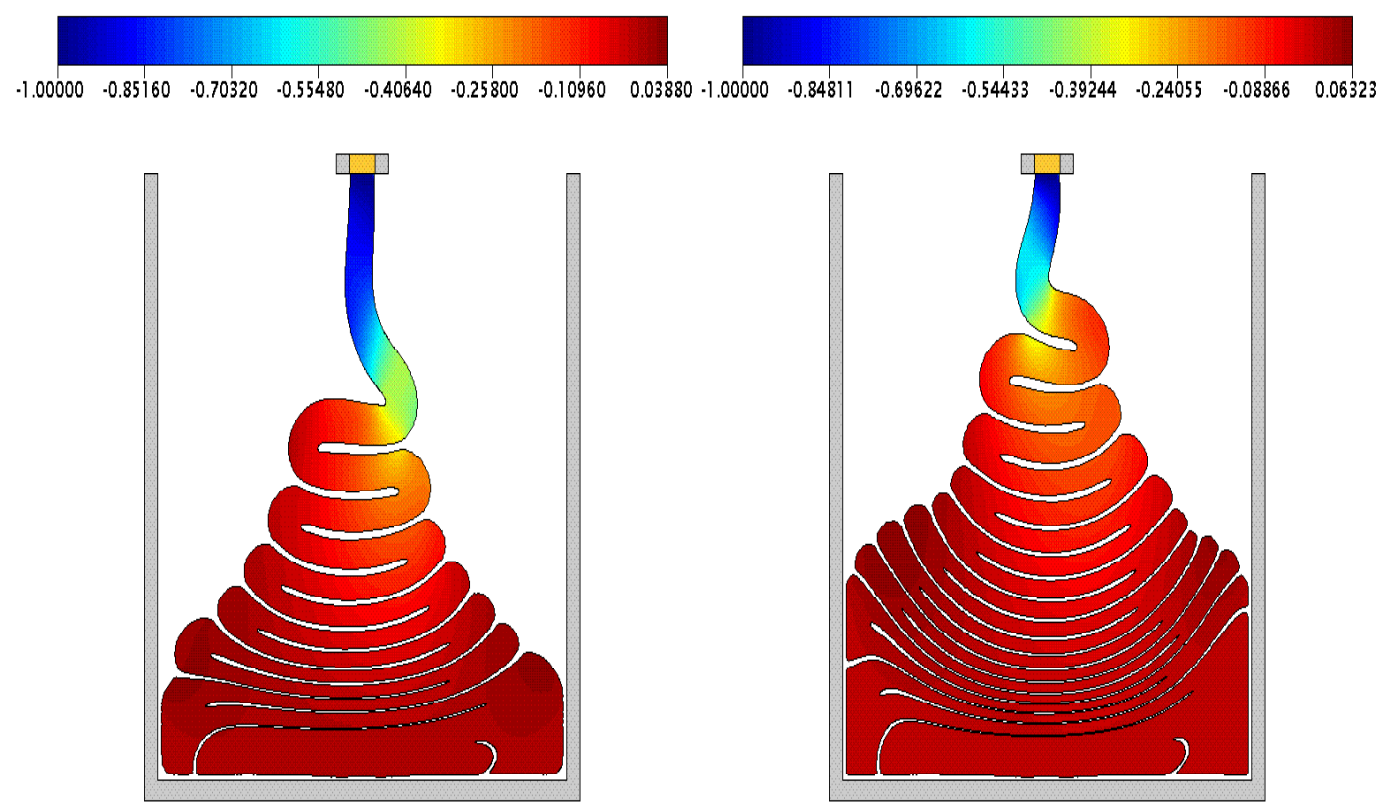

Figura 6.21: Continuação da Figura 6.20. 


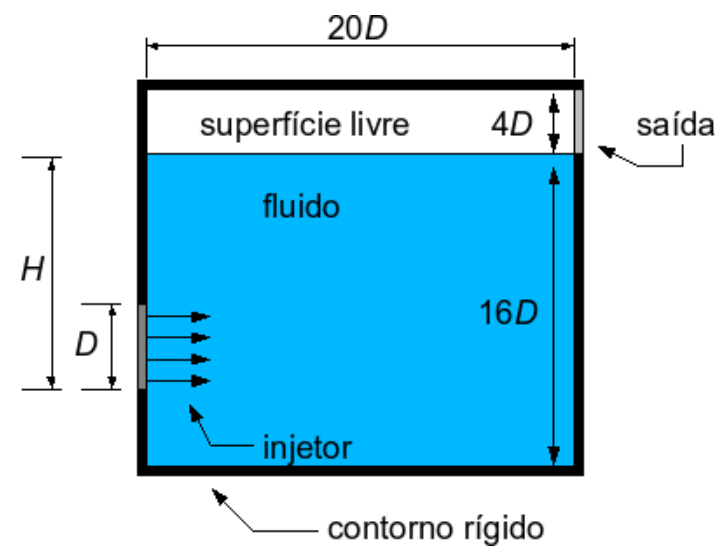

Figura 6.22: Geometria do problema de um jato penetrando num corpo de fluido.

As soluções numéricas obtidas para o campo de pressão e para as componentes $u$ e $v$ da velocidade estão mostradas nas Figuras 6.26, 6.27 e 6.28, respectivamente. Nessas figuras, percebe-se, inicialmente, a formação de um par de vórtices com orientações contrárias. Esse par de vórtices se movimenta em direção à superfície livre, provocando a formação de grandes ondulações na mesma. Grandes estruturas turbilhonares, ao longo de todo o domínio do problema, podem ser visualizadas no tempo final $t=3 \mathrm{~s}$. Em suma, pode-se notar que pequenos e grandes vórtices apareceram no escoamento transiente simulado, provocando fortes efeitos na superfície livre. É importante salientar que os resultados numéricos obtidos nesta aplicação estão em concordância com as soluções numéricas de Brandi [17] e Ferreira et al. [44]. 
(a) ALUS
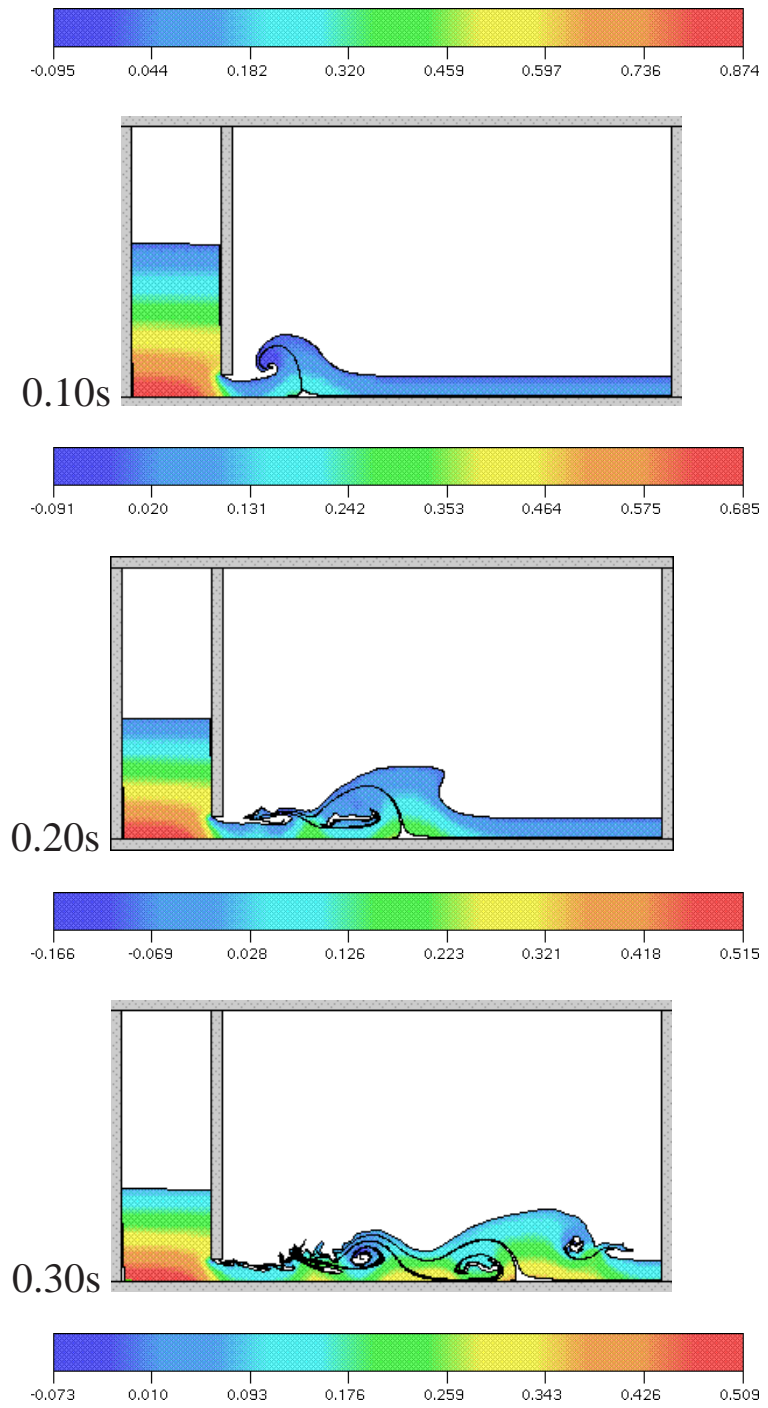

$0.40 \mathrm{~s}$

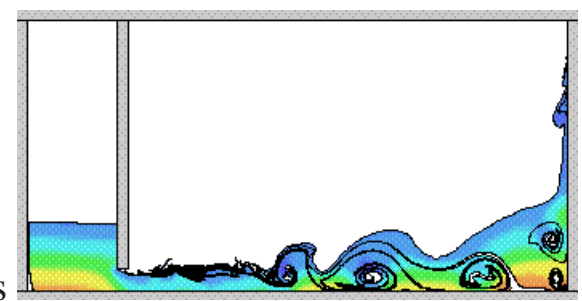

(b) TOPUS

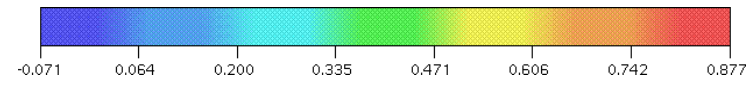

$0.10 \mathrm{~s}$
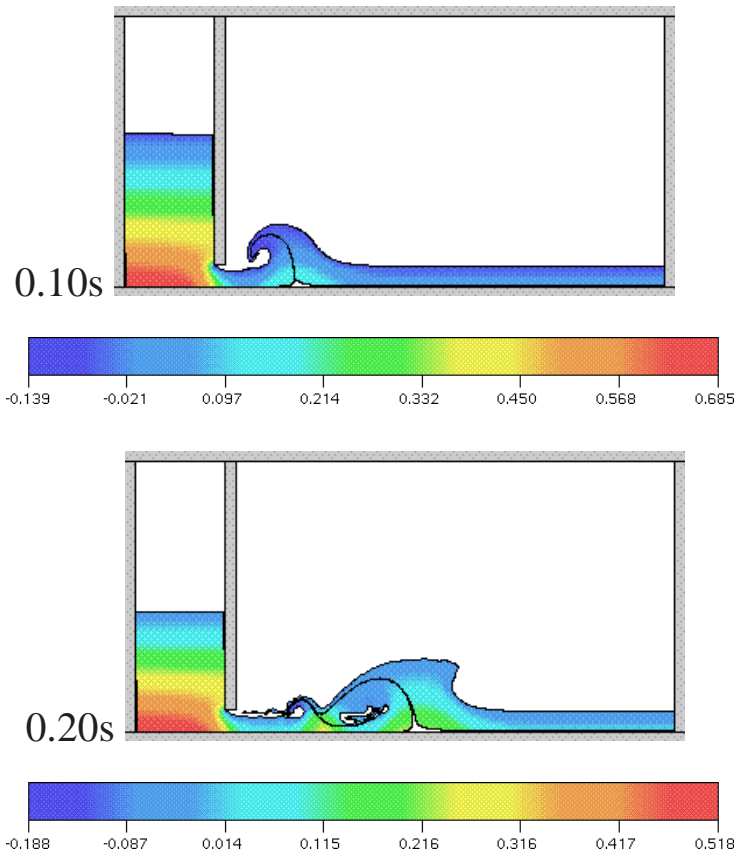

$0.30 \mathrm{~s}$
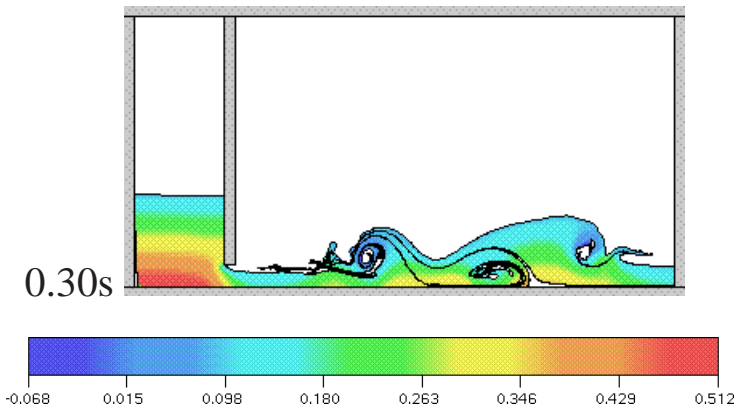

$0.40 \mathrm{~s}$

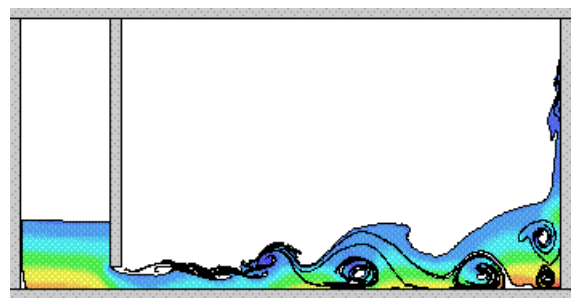

Figura 6.23: Evolução dos contornos do campo de pressão do problema "sluice gate". 
(a) ALUS
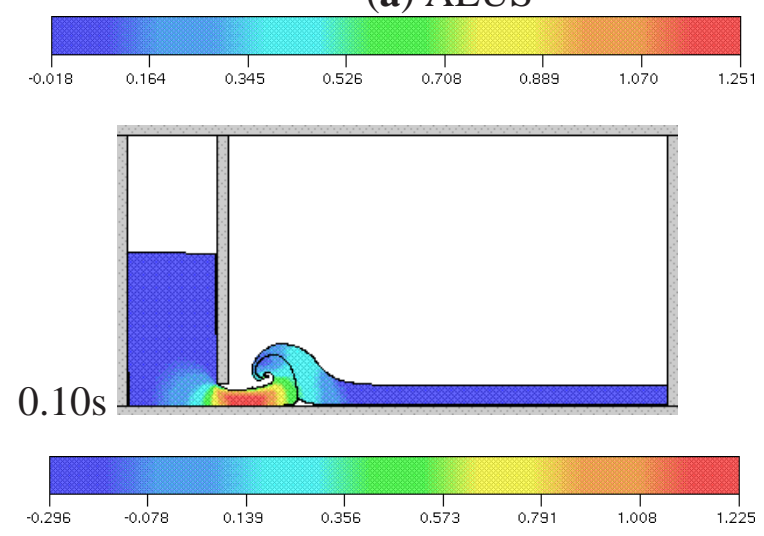

$0.20 \mathrm{~s}$
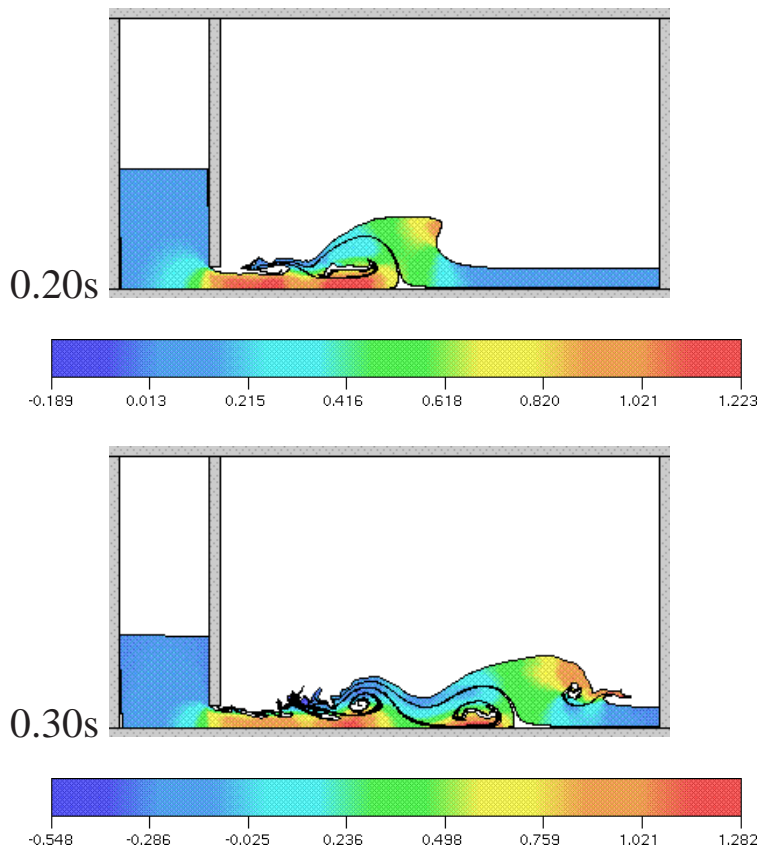

$0.40 \mathrm{~s}$

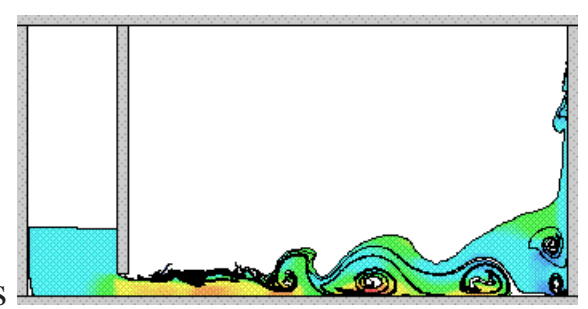

(b) TOPUS

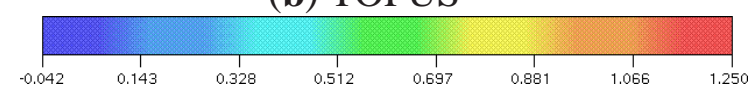

$0.10 \mathrm{~s}$
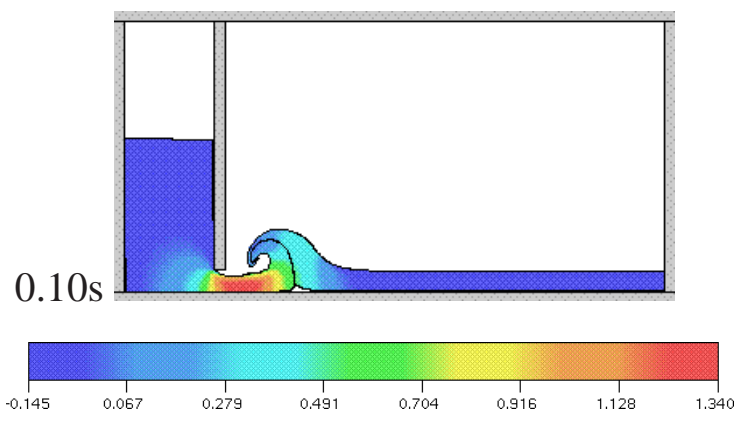

$0.20 \mathrm{~s}$
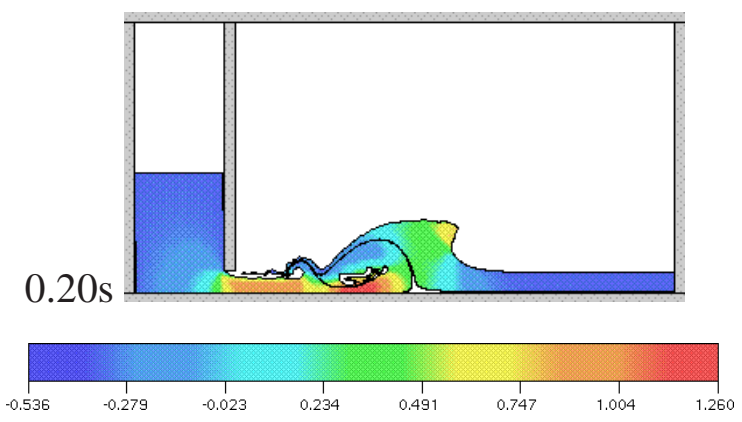

$0.30 \mathrm{~s}$
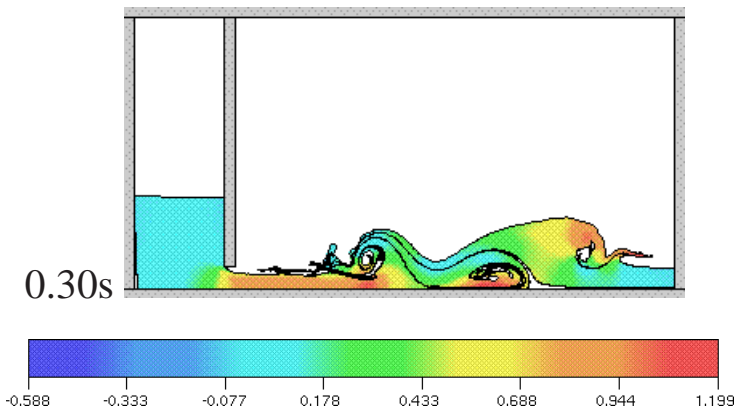

$0.40 \mathrm{~s}$

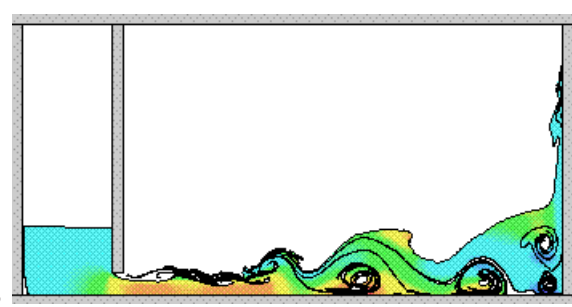

Figura 6.24: Evolução dos contornos da componente de velocidade $u$ do problema "sluice gate". 
(a) ALUS
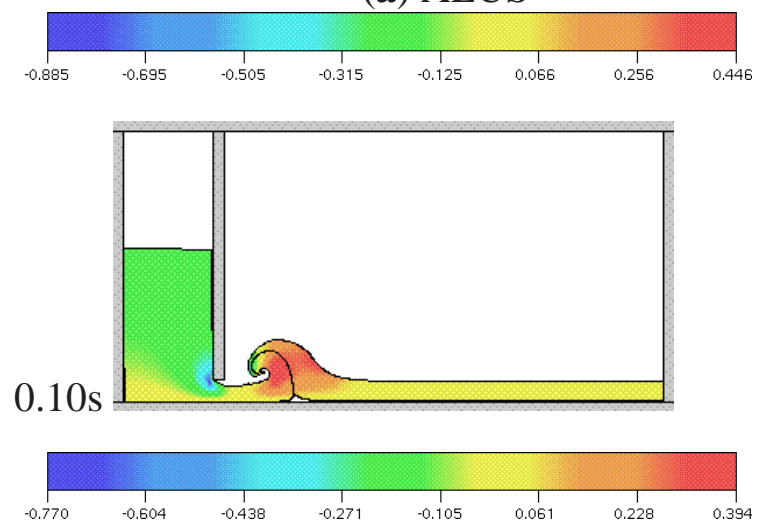

$0.20 \mathrm{~s}$
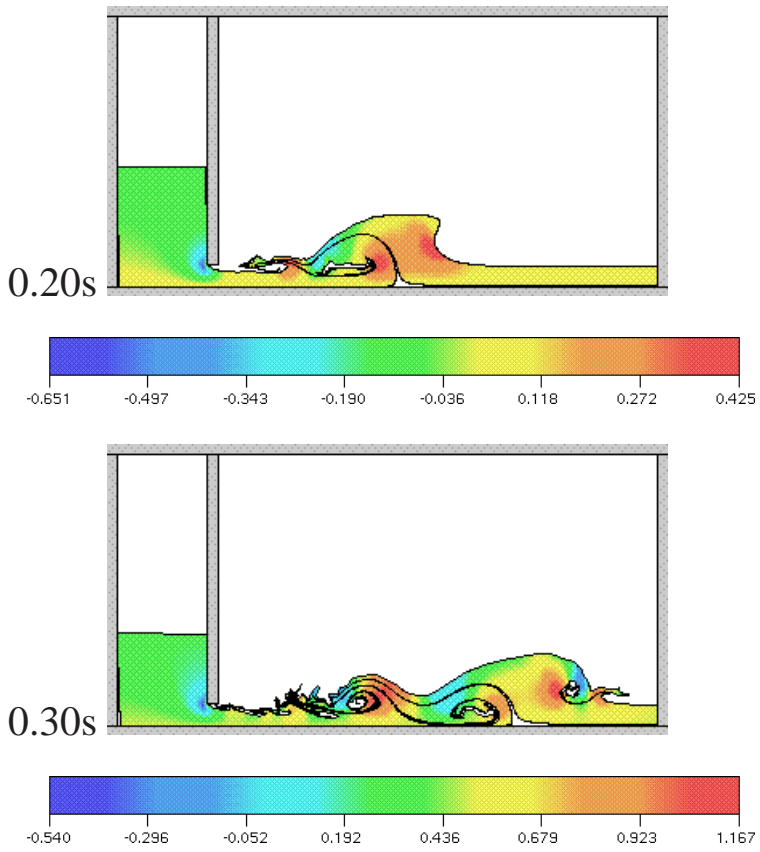

$0.40 \mathrm{~s}$

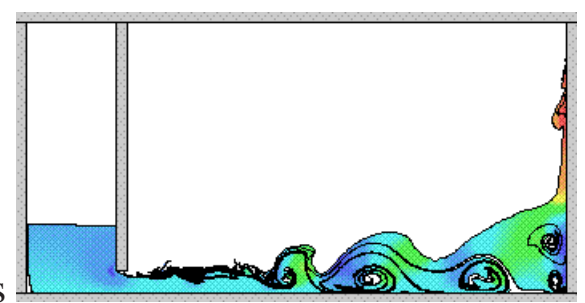

(b) TOPUS

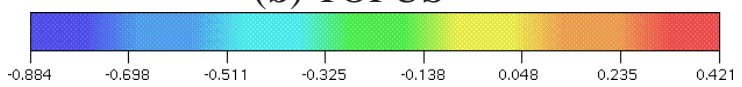

$0.10 \mathrm{~s}$
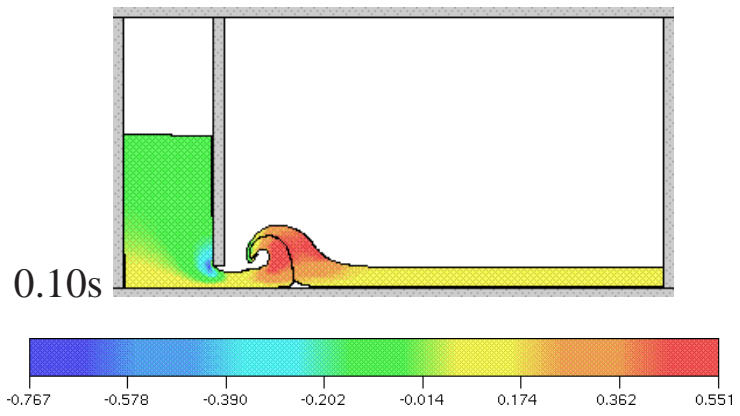

$0.20 \mathrm{~s}$
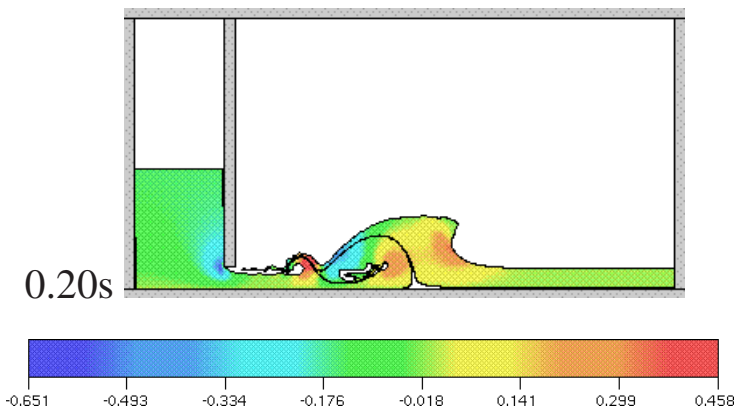

$0.30 \mathrm{~s}$
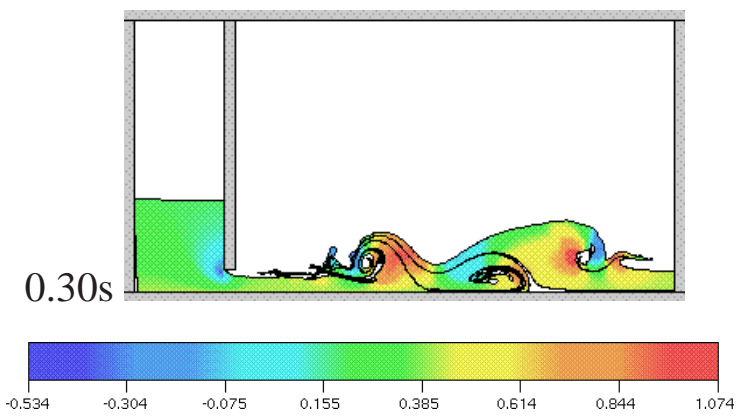

$0.40 \mathrm{~s}$

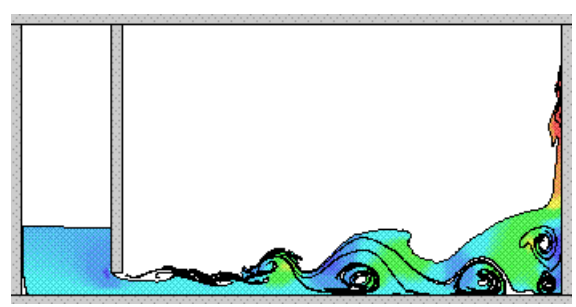

Figura 6.25: Evolução dos contornos da componente de velocidade $v$ do problema "sluice gate". 
(a) ALUS
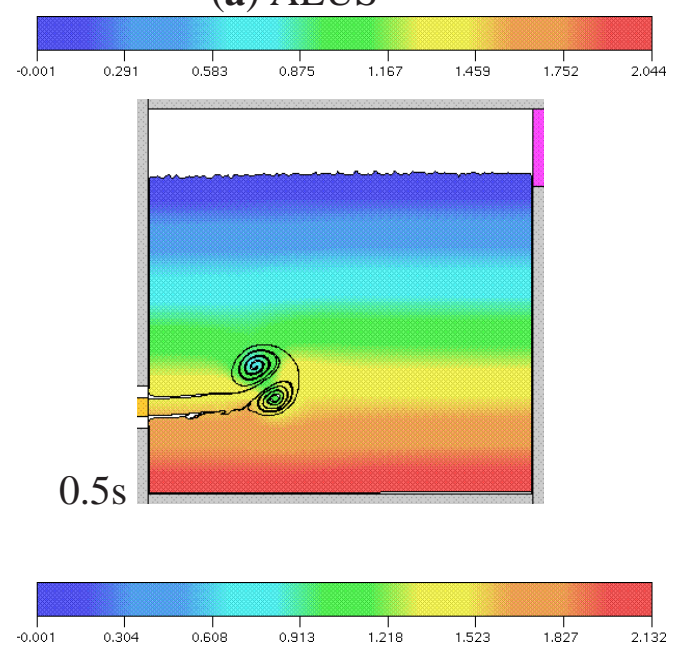

$1.0 \mathrm{~s}$
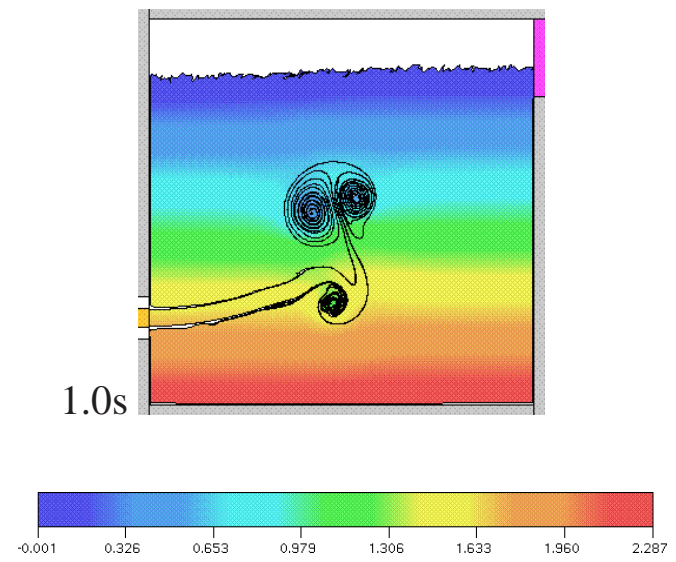

$2.0 \mathrm{~s}$
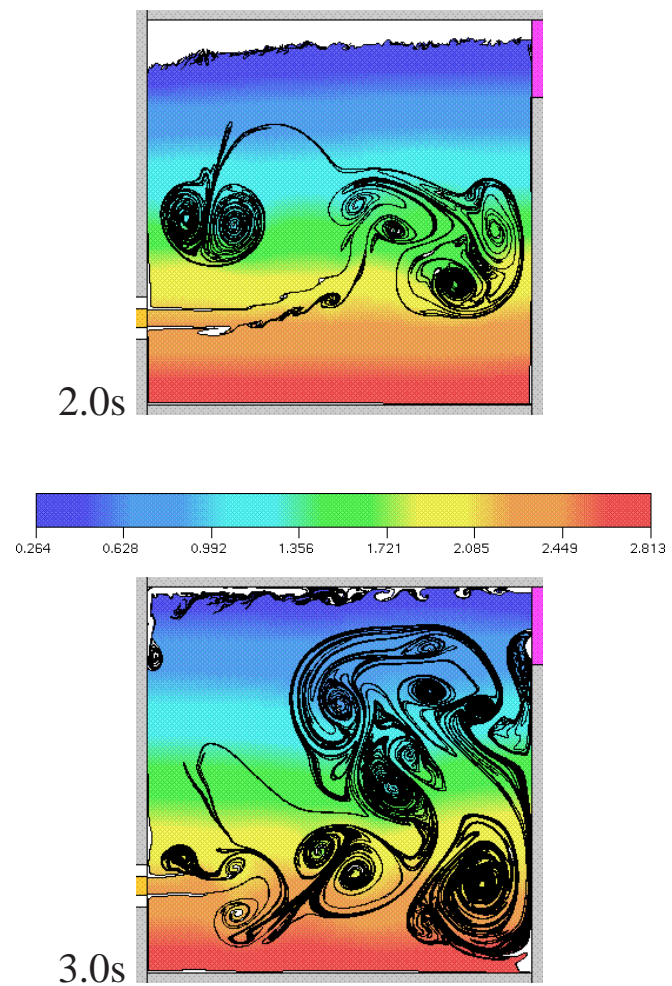

(b) TOPUS
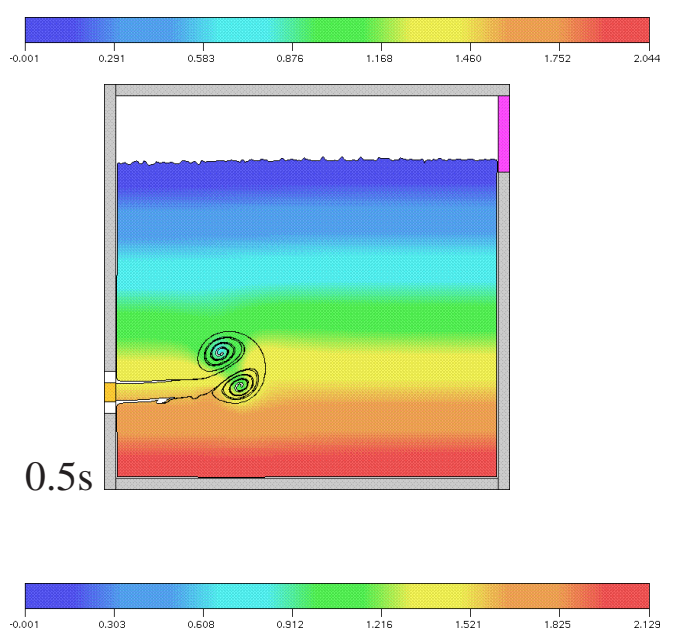

$1.0 \mathrm{~s}$

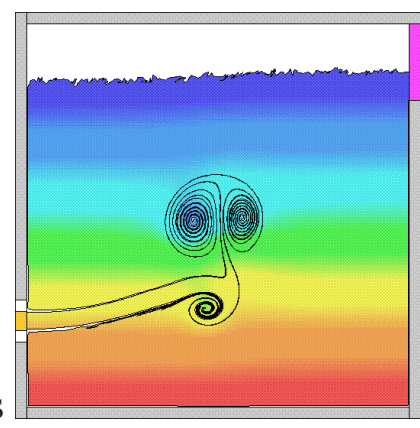

$2.0 \mathrm{~s}$
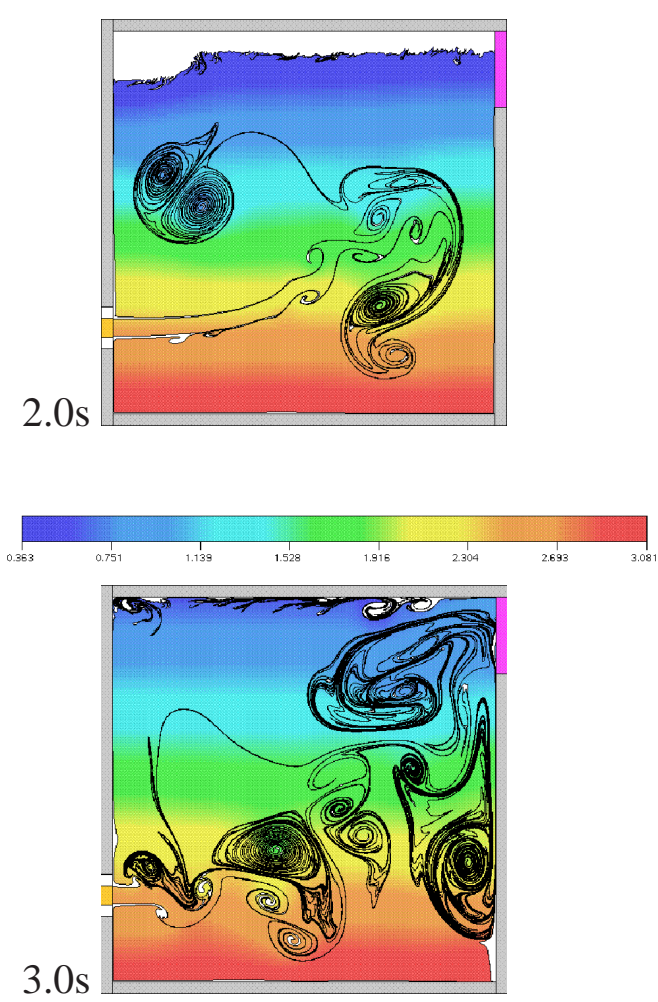

Figura 6.26: Contornos de pressão de um jato penetrando numa porção de fluido em repouso. 
(a) ALUS
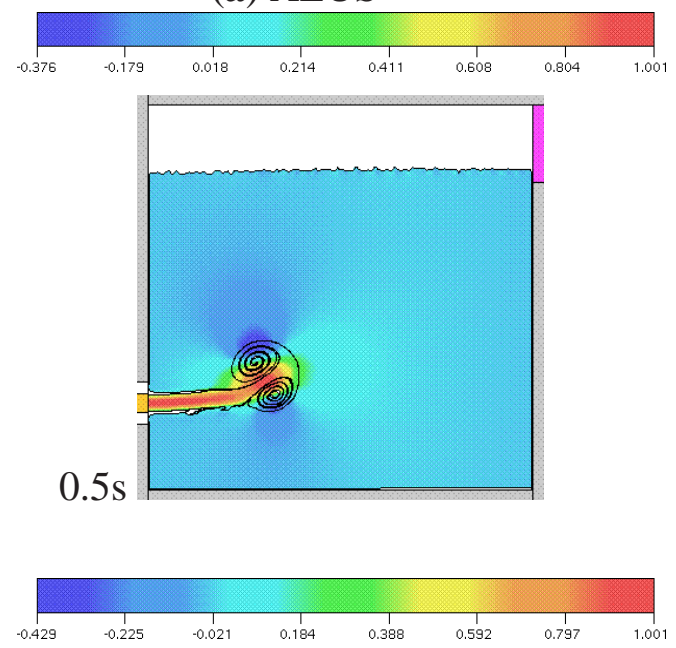

$1.0 \mathrm{~s}$
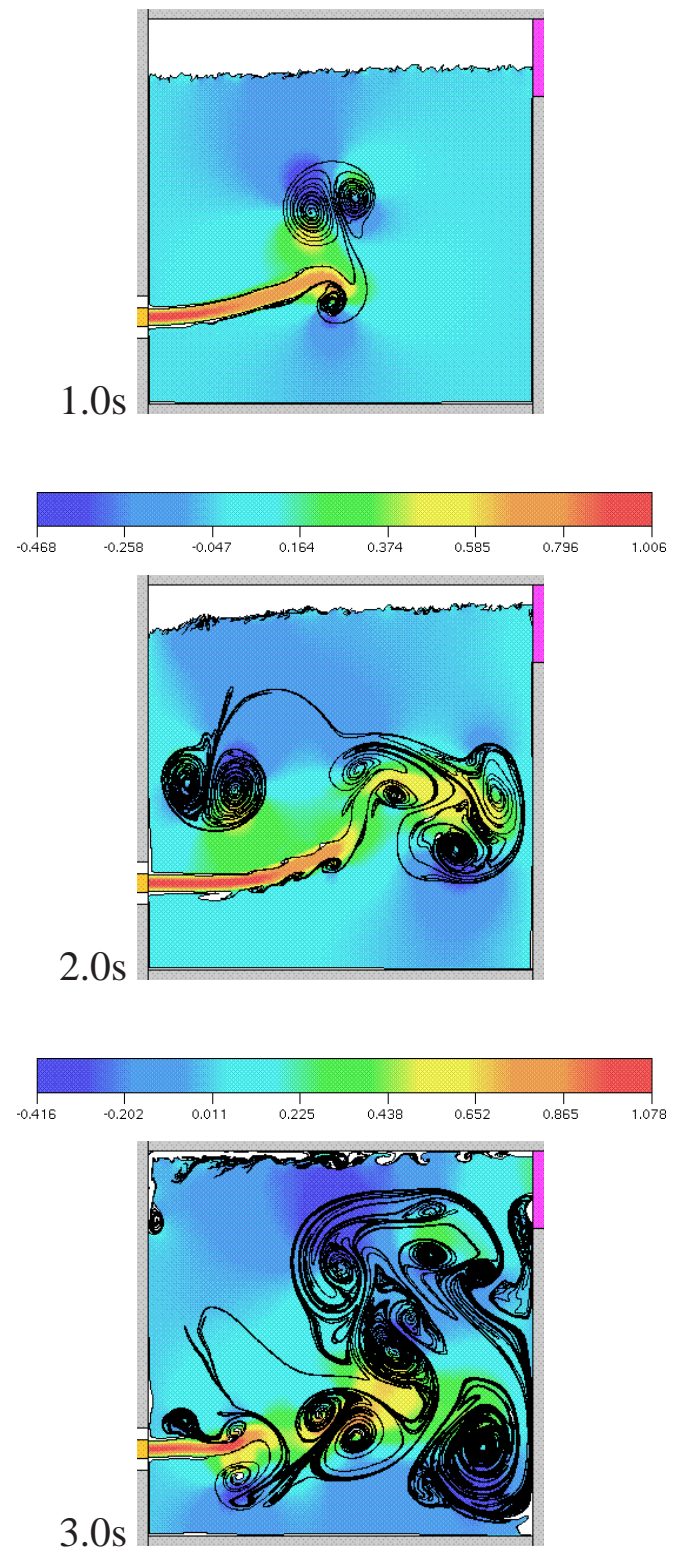

(b) TOPUS
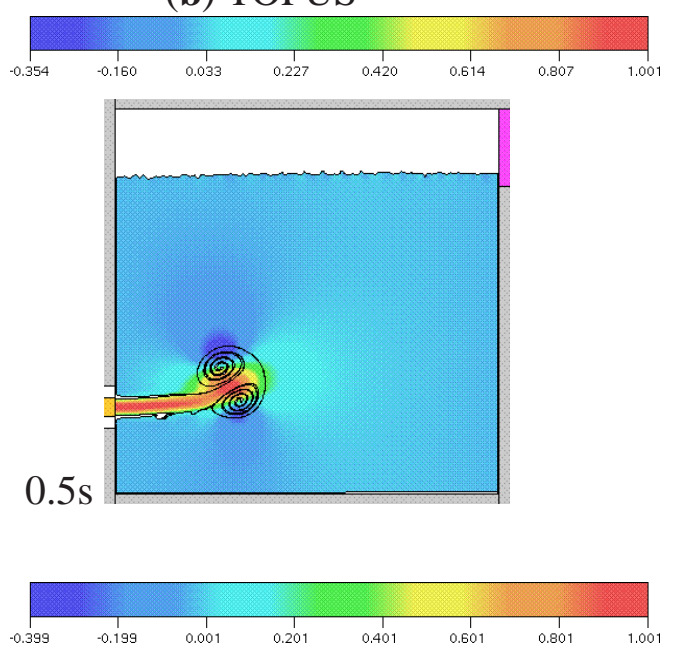

$1.0 \mathrm{~s}$
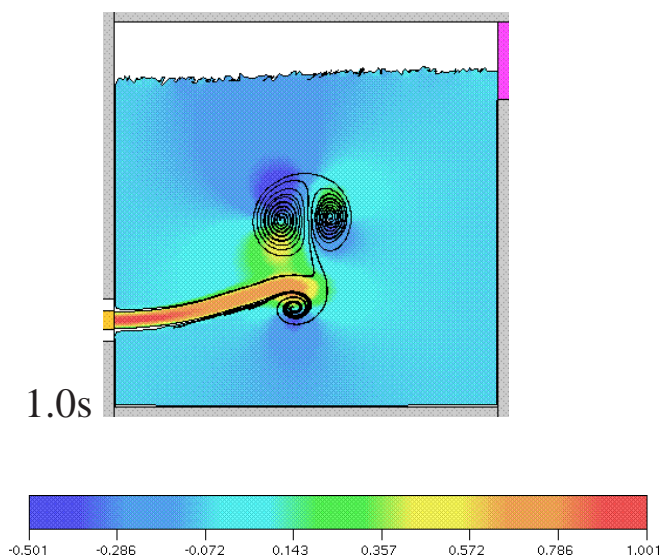

$2.0 \mathrm{~s}$
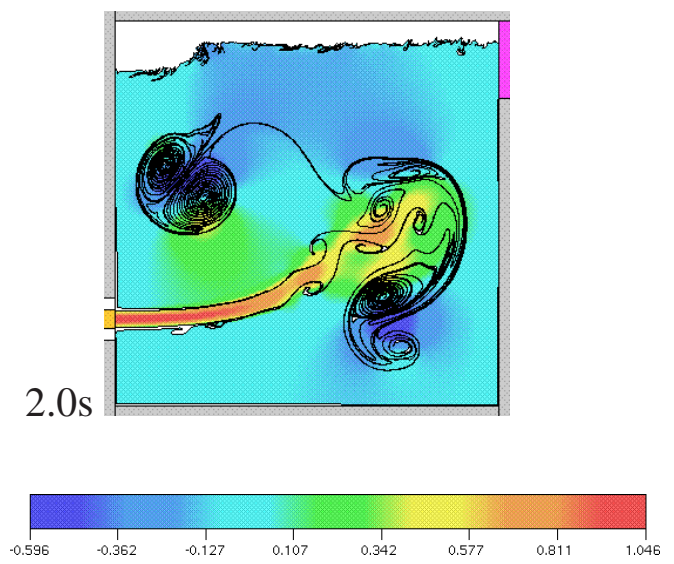

$3.0 \mathrm{~s}$

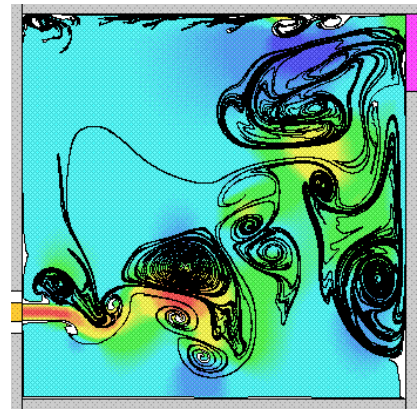

Figura 6.27: Contornos da componente $u$ da velocidade de um jato penetrando numa porção de fluido em repouso. 
(a) ALUS
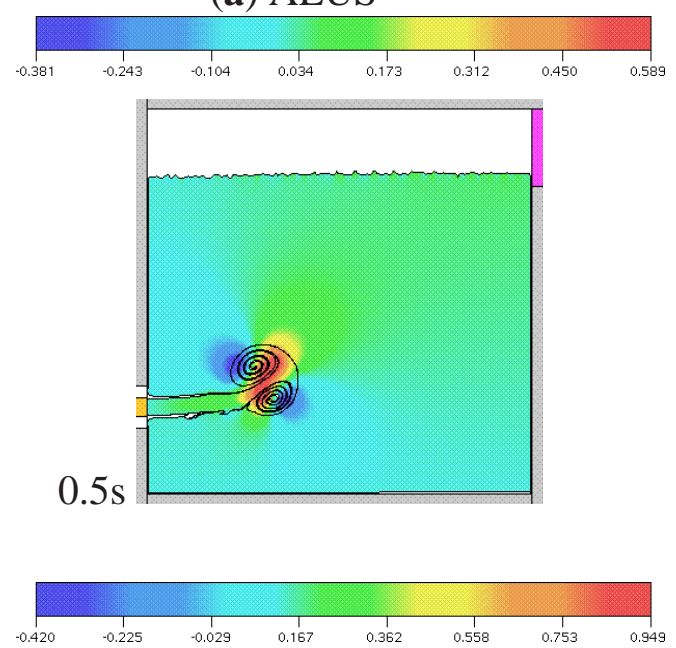

$1.0 \mathrm{~s}$
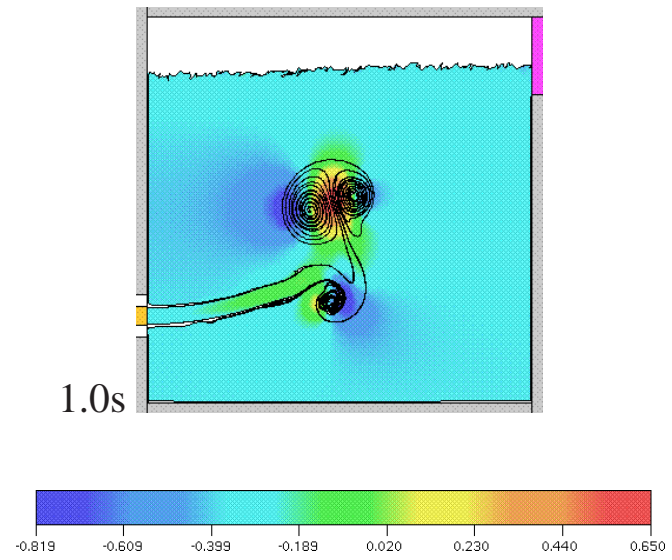

$2.0 \mathrm{~s}$
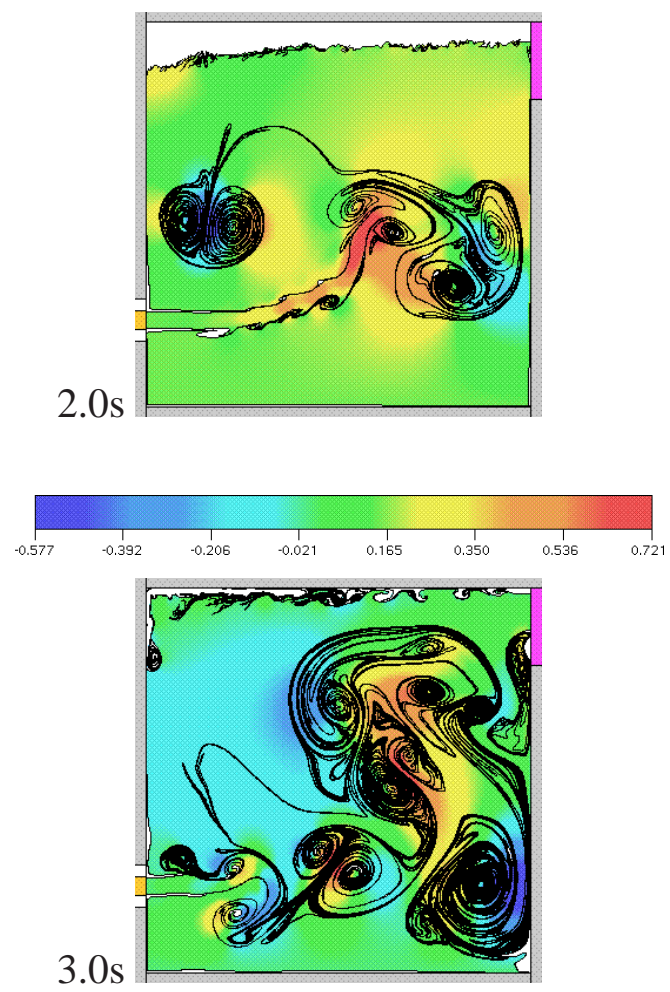

(b) TOPUS
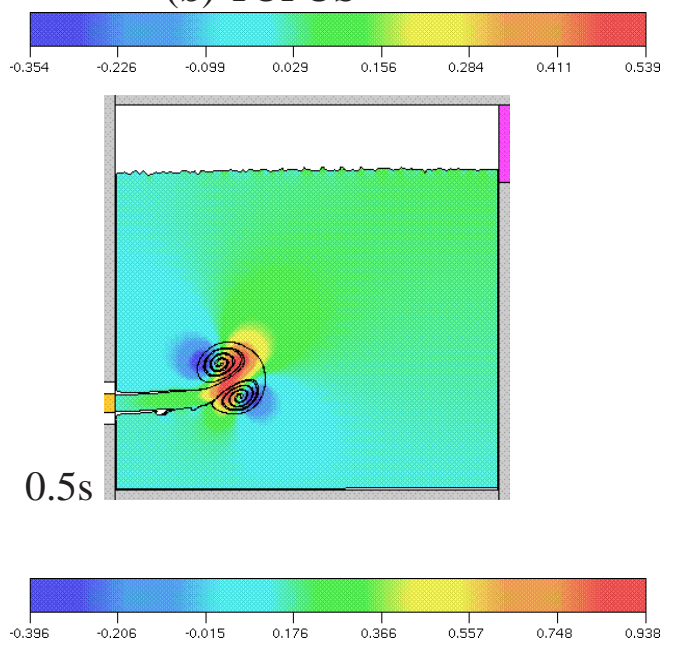

$1.0 \mathrm{~s}$
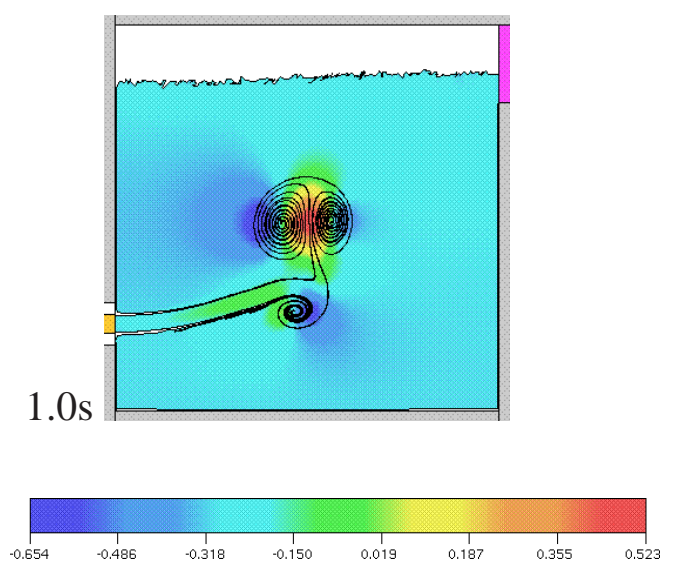

$2.0 \mathrm{~s}$
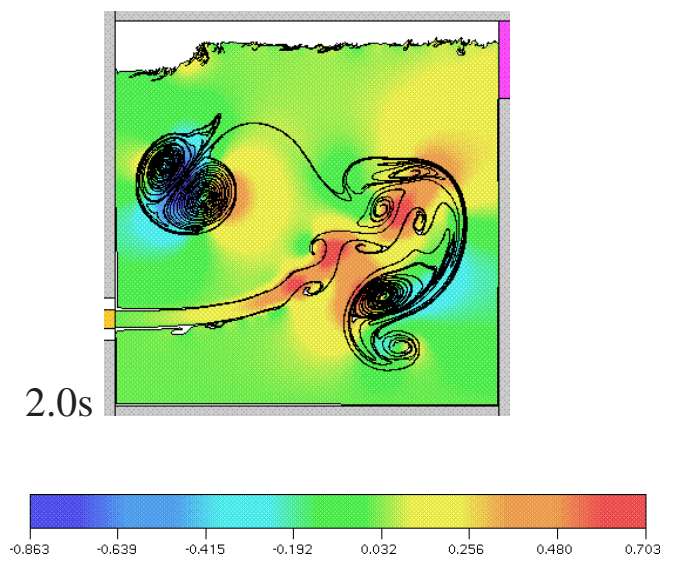

$3.0 \mathrm{~s}$

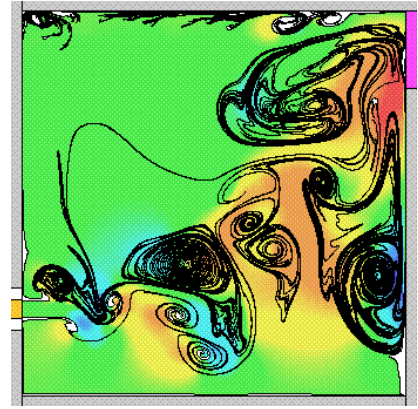

Figura 6.28: Contornos da componente $v$ da velocidade de um jato penetrando numa porção de fluido em repouso. 


\subsection{Problemas Compressíveis}

Nesta seção, são apresentados resultados numéricos de escoamentos compressíveis ao redor de aerofólios obtidos nas simulações com os limitadores de fluxo dos esquemas TOPUS e van Albada [150]. A formulação matemática do limitador TOPUS adotada nestes problemas compressíveis é descrita no apêndice D. Não se utilizou o limitador do esquema ALUS, pois ele é uma função contínua por partes (não suave). Limitadores diferenciáveis, como o TOPUS e o van Albada, permitem transições suaves entre regiões do escoamento e outras vantagens (ver Bigarella [14]). A modelagem matemática dos escoamentos aerodinâmicos aqui abordados é definida pelas equações de Euler 2D delineadas na seção 2.5. Para obtenção das soluções numéricas, as reconstruções do método Roe de segunda ordem [29, 65, 117] em variáveis conservadas (Cons) e primitivas (Prim) são aplicadas. Uma malha estruturada do tipo "O" $[65,66]$ com $251 \times 151$ volumes de controle foi adotada nas simulações. As soluções numéricas descritas abaixo foram parcialmente publicadas em anais de eventos e periódico (ver referências [30, 103, 109]).

\subsubsection{Diamante}

Resultados numéricos da simulação do escoamento supersônico ao redor de um aerofólio diamante [6] são aqui delineados. Os dados do modelo simulado são o choque oblíquo com ângulo de deflecção $10^{\circ}$ e número de Mach 1.5. As soluções numéricas fornecidas com a utilização dos limitadores de fluxo TOPUS e van Albada para o ângulo do choque, cujo resultado teórico é $56.5^{\circ}$ (ver [6]), são apresentadas na Tabela 6.7. Nota-se por essa tabela que a estimativa para o ângulo do choque obtida com o TOPUS, em ambas as reconstruções, está bem próxima daqueles de van Albada e teórico. A convergência do processo numérico usando o TOPUS é mostrada na Figura 6.29 , onde pode-se ser visto que ela não é afetada por esse limitador em ambas as reconstruções do método Roe [117].

Tabela 6.7: Comparação entre os resultados numéricos e teórico da inclinação da onda de choque oblíqua.

\begin{tabular}{|c||c||c|}
\hline Limitador & Reconstrução de variáveis & Inclinação da onda de choque \\
\hline & Conservadas & $56.74^{\circ}$ \\
van Albada & Primitivas & $56.79^{\circ}$ \\
\hline \multirow{2}{*}{ TOPUS } & Conservadas & $57.53^{\circ}$ \\
\hline \hline Resultado teórico [6] & Primitivas & $57.04^{\circ}$ \\
\hline
\end{tabular}

\subsubsection{RAE 2822}

São apresentados agora os resultados numéricos obtidos na simulação do escoamento transônico ao redor de um aerofólio RAE 2822 a Mach 0.725 e ângulo de ataque $2.310^{\circ}$ [160]. A Figura 6.30a mostra a convergência do método numérico com o limitador TOPUS nas duas reconstruções do método Roe [29, 65, 117], onde $\log ($ Res ) denota o logaritmo do resíduo calculado em cada iteração do método. Observa-se por essa figura que o método numérico convergiu em ambas as reconstruções. A Figura $6.30 \mathrm{~b}$ mostra a comparação entre os resultados numéricos com TOPUS e van Albada e o dado experimental de Slater [128] para o coeficiente de pressão (Cp), onde x/c denota a razão entre as coordenadas dos pontos do domínio e o comprimento da corda do perfil do 


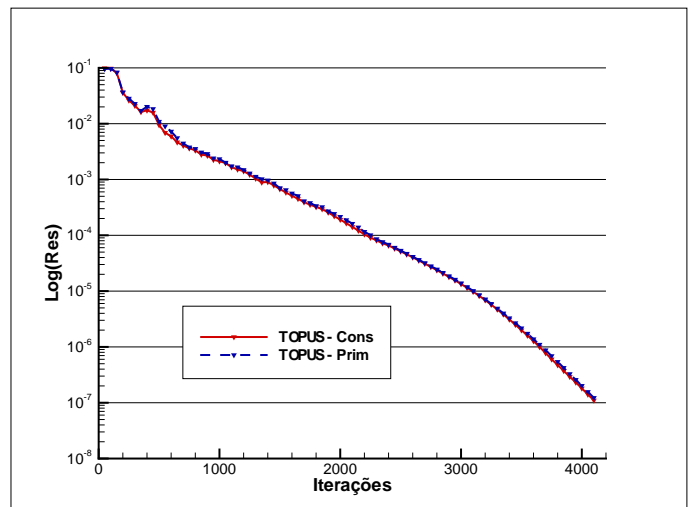

Figura 6.29: Convergência do método numérico na simulação do escoamento supersônico ao redor de um aerofólio diamante.

aerofólio $(\mathrm{x} \in[0, \mathrm{c}])$. Vê-se nessa figura que os resultados com TOPUS e van Albada são, praticamente, idênticos, e os quais estão em concordância com o experimento de Slater. As distribuições de pressão e Mach, obtidos com o TOPUS, ao redor do aerofólio são ilustradas nas Figuras 6.31.

Os perfis de pressão, número de Mach e densidade simulados com o TOPUS nas duas reconstruções estão apresentados nas Figuras 6.32, 6.33 e 6.34, respectivamente. Em particular, os detalhes da posição e intensidade do choque podem ser visualizados nas Figuras 6.32b, 6.33b e 6.34b. Observa-se por essas figuras que ambas as reconstruções são muito similares.

(a)

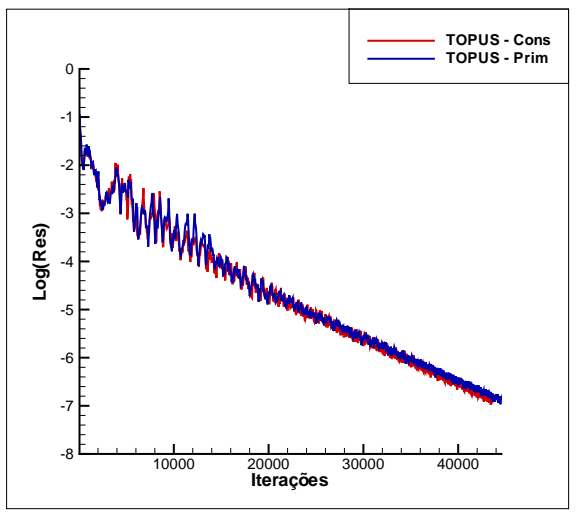

(b)

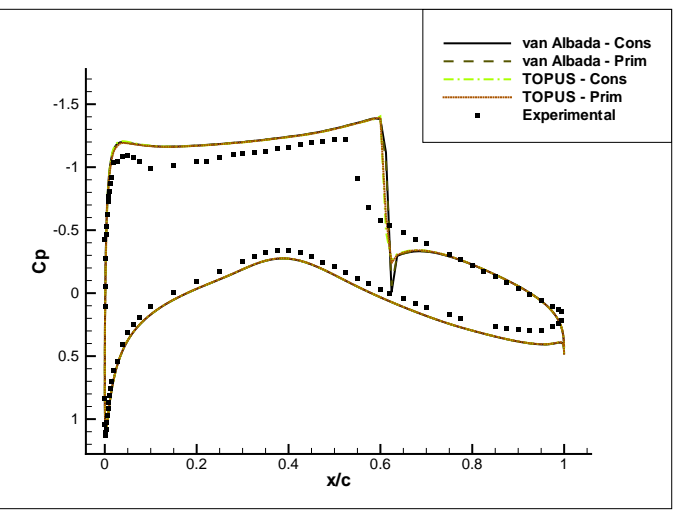

Figura 6.30: Simulação do escoamento transônico ao redor do aerofólio RAE 2822: (a) análise de convergência; (b) comparação entre os resultados numéricos e dado experimental para o coeficiente de pressão Cp. 
(a) Pressão (P)

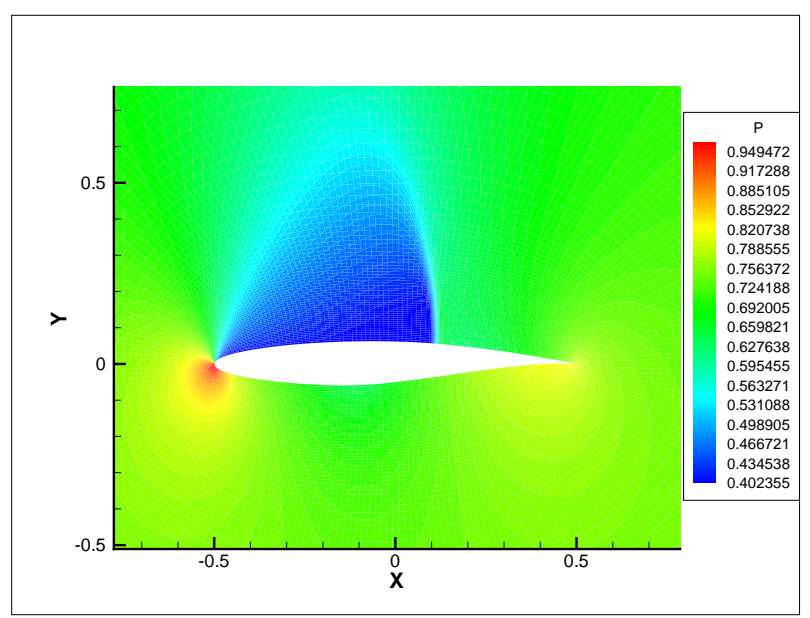

(b) Mach

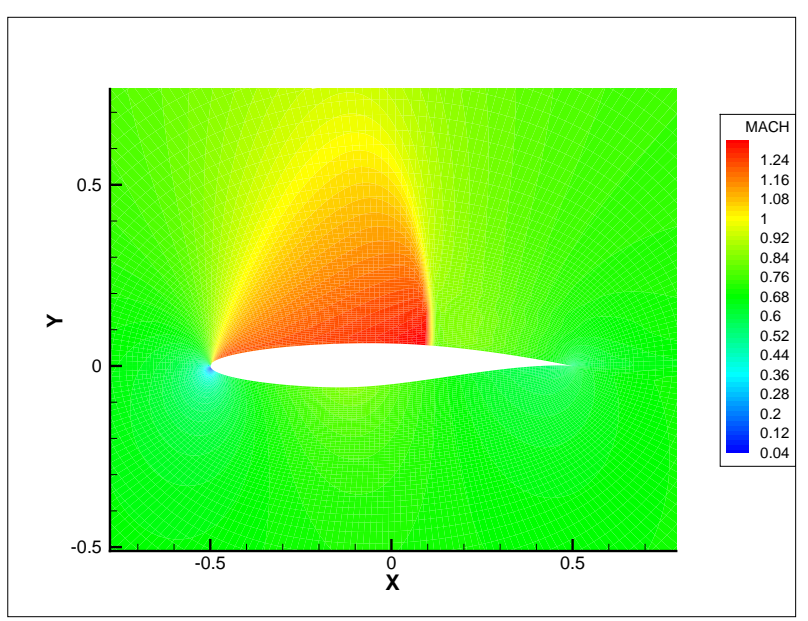

Figura 6.31: Resultados numéricos da distribuição de pressão e Mach ao redor do aerofólio RAE 2822.

(a)

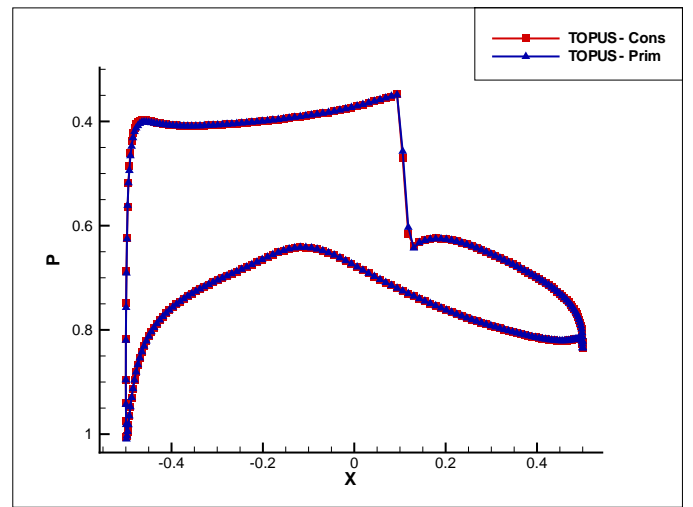

(b)

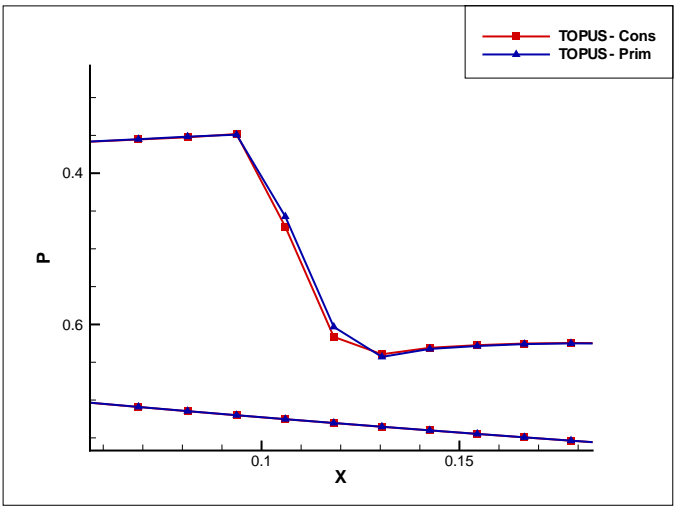

Figura 6.32: Resultados numéricos da distribuição da pressão P: (a) ao redor do aerofólio RAE 2822; (b) detalhe da região do choque.

(a)

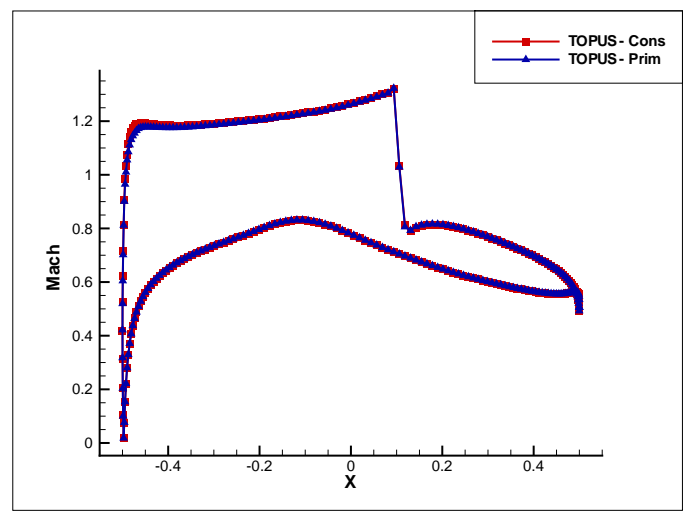

(b)

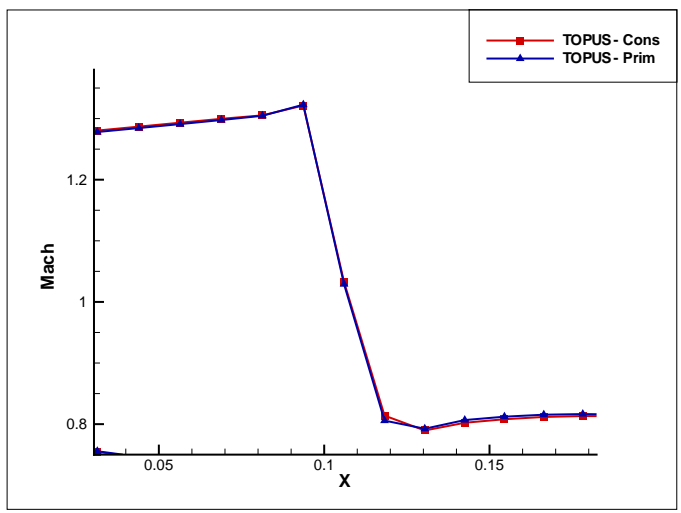

Figura 6.33: Resultados numéricos da distribuição Mach: (a) ao redor do aerofólio RAE 2822; (b) detalhe da região do choque. 
(a)

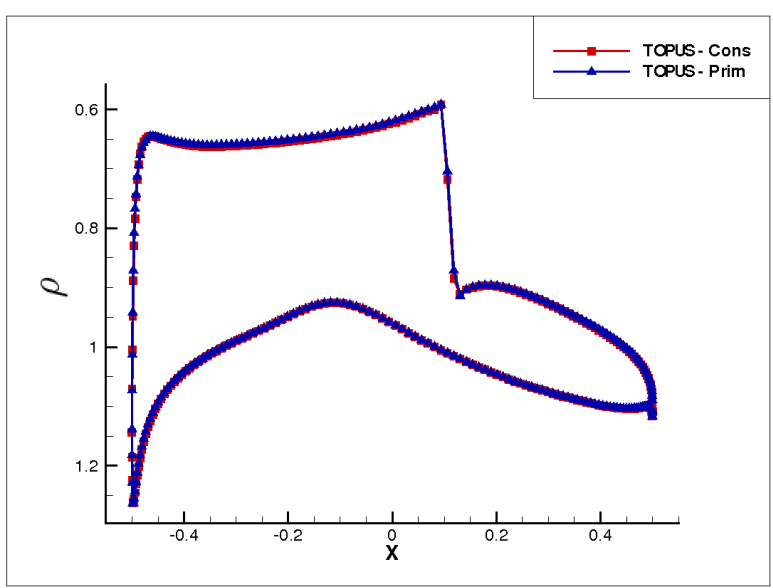

(b)

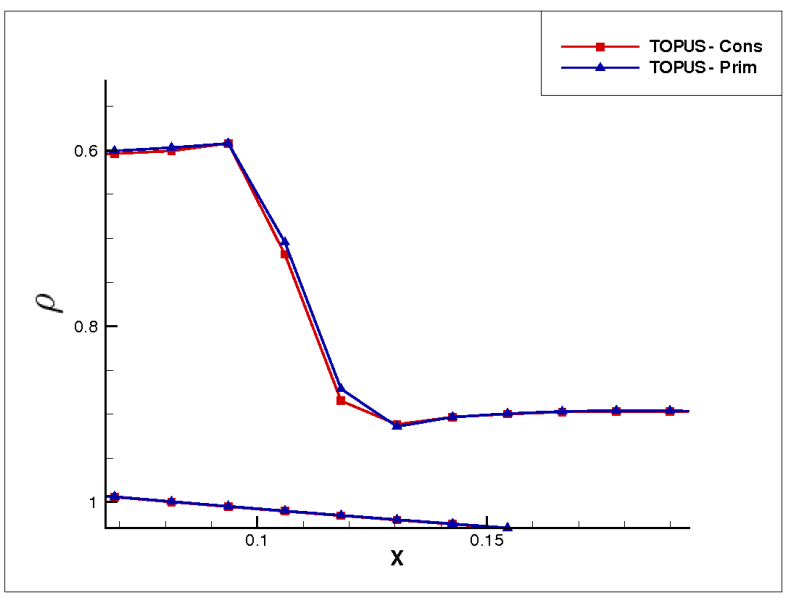

Figura 6.34: Resultados numéricos da distribuição de densidade $\rho$ : (a) ao redor do aerofólio RAE 2822; (b) detalhe da região do choque.

\subsubsection{NACA 0012}

Resultados numéricos obtidos com os limitadores TOPUS e van Albada para o escoamento ao redor do aerofólio NACA 0012 [160] são mostrados nesta subseção. As simulações foram realizadas utilizando três combinações de Mach e ângulo de ataque: Mach 0.3 com ângulo $0^{\circ}$; Mach 0.5 com ângulo $8^{\circ}$; e Mach 0.75 com ângulo $4^{\circ}$.

Nas Figuras 6.35, 6.36 e 6.37 estão mostradas as comparações dos dados numéricos obtidos (em variáveis primitivas e conservadas) e experimentais de Thibert et al. [143] para o coeficiente de pressão $\mathrm{Cp}$ nas três combinações de Mach e ângulo de ataque descritas acima. De maneira geral, observa-se por essas figuras que o esquema TOPUS simulou muito bem o problema, sendo difícil dizer qual esquema forneceu o melhor resultado: se o van Albada ou se o TOPUS.

(a) Variáveis conservadas

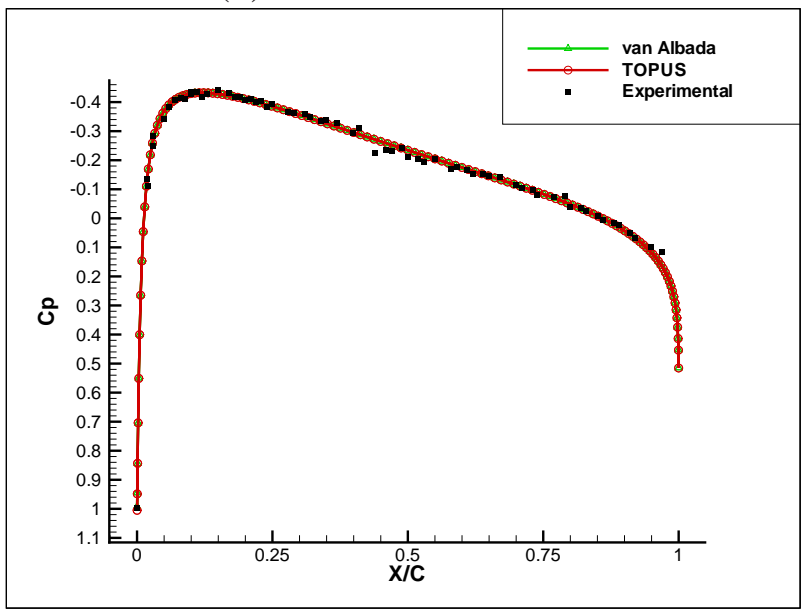

(b) Variáveis primitivas

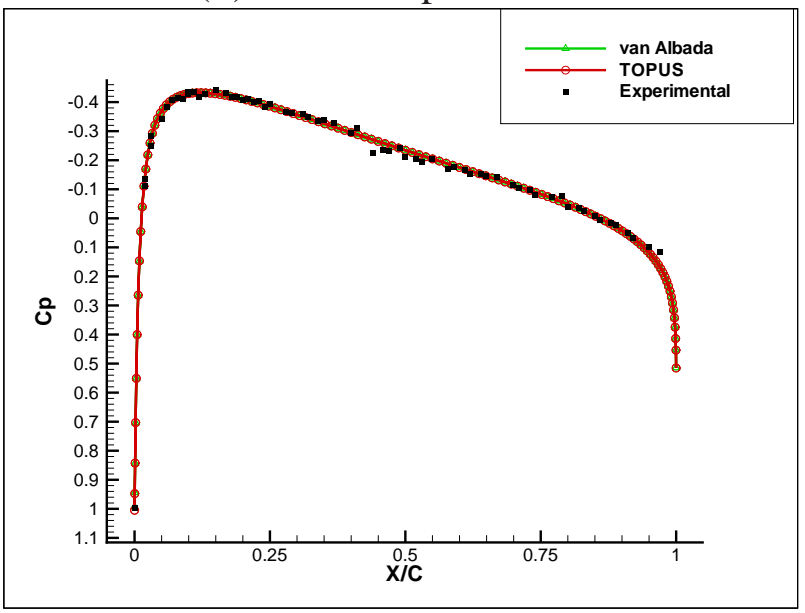

Figura 6.35: Comparação entre os resultados numéricos e dado experimental para o coeficiente de pressão Cp ao redor do aerofólio NACA 0012 a Mach 0.3 e ângulo de ataque $0^{\circ}$. 
(a) Variáveis conservadas

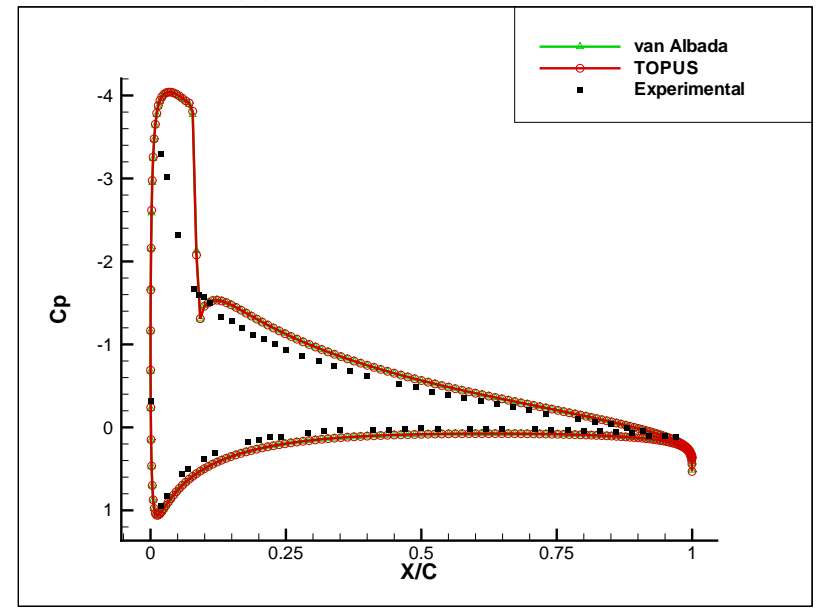

(b) Variáveis primitivas

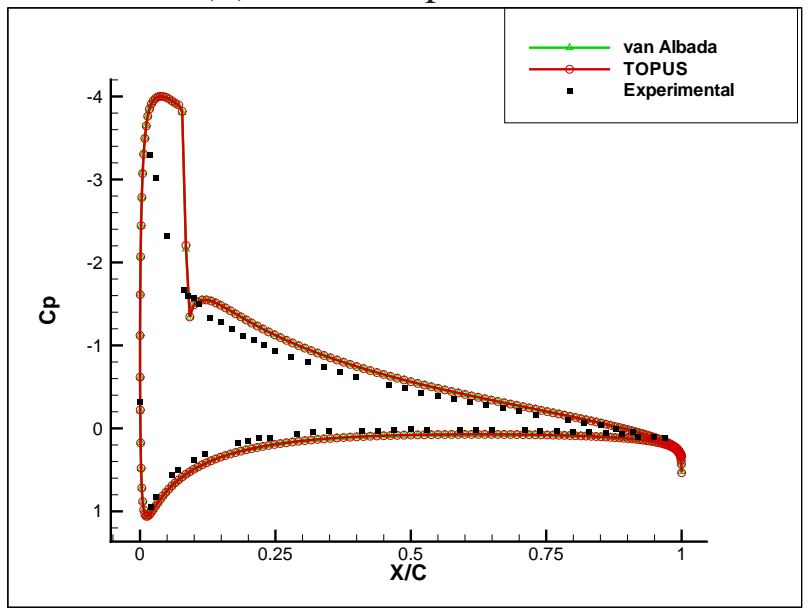

Figura 6.36: Comparação entre os resultados numéricos e dado experimental para o coeficiente de pressão Cp ao redor do aerofólio NACA 0012 a Mach 0.5 e ângulo de ataque $8^{\circ}$.

(a) Variáveis conservadas

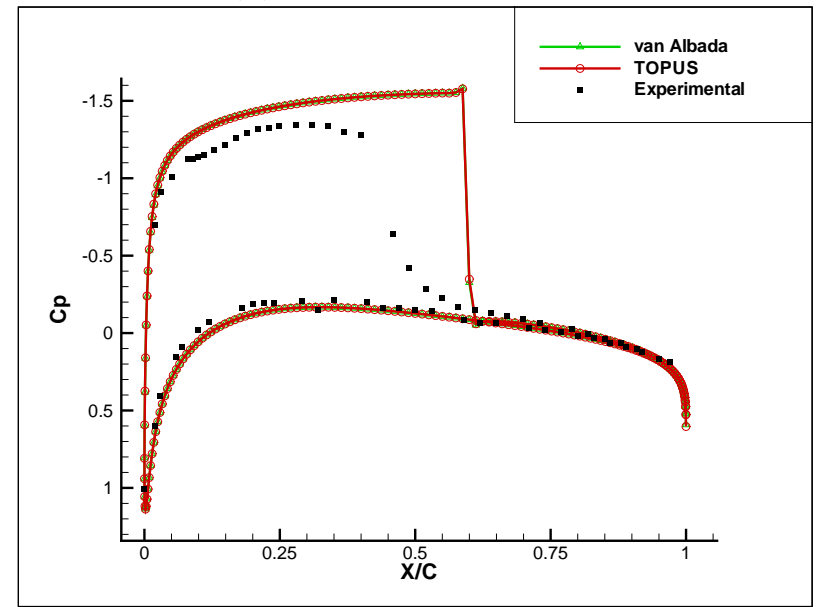

(b) Variáveis primitivas

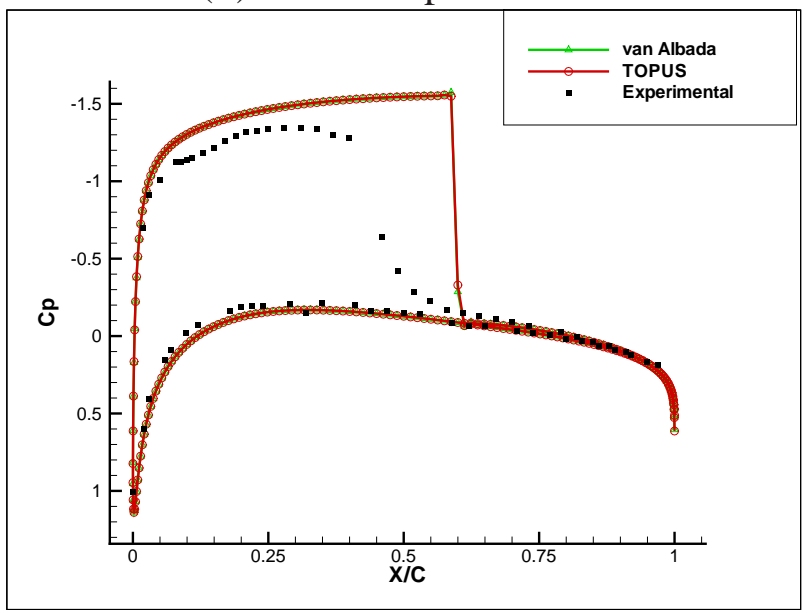

Figura 6.37: Comparação entre os resultados numéricos e dado experimental para o coeficiente de pressão Cp ao redor do aerofólio NACA 0012 a Mach 0.75 e ângulo de ataque $4^{\circ}$. 


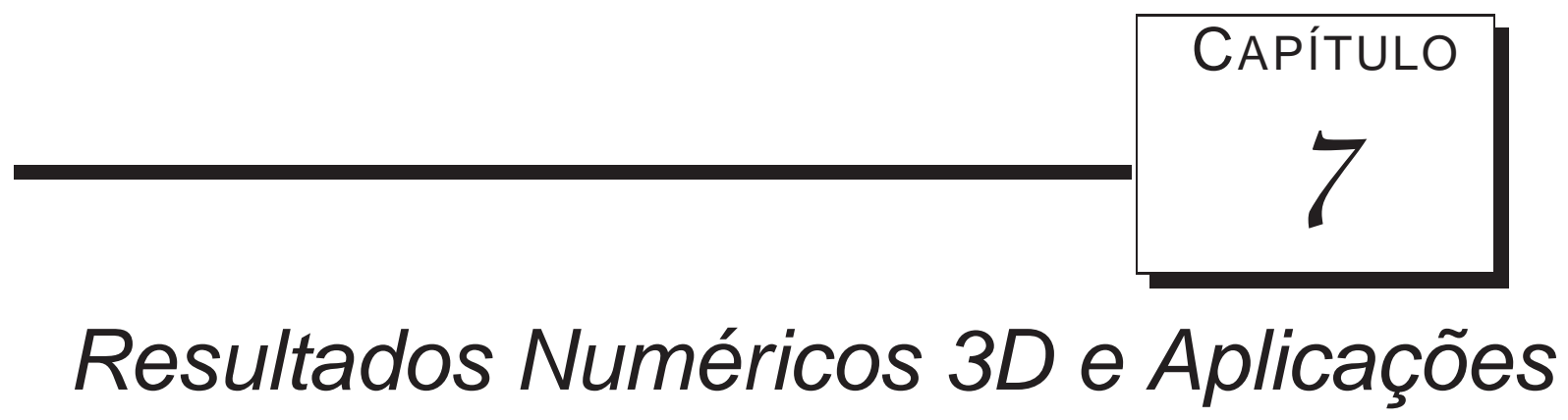

Para demonstrar a aplicabilidade dos esquemas "upwind" de alta resolução ALUS e TOPUS em problemas altamente complexos, neste capítulo são apresentadas simulações numéricas, sem modelagem da turbulência, de problemas de escoamentos incompressíveis transientes 3D com superfícies livres móveis a vários Reynolds. O sistema de simulação "Freeflow" (versão 3D) de Castelo et al. [20], equipado com os esquemas ALUS e TOPUS, foi utilizado para as simulações. Quatro tipos de problemas são considerados: colapso de um bloco de fluido; ressalto hidráulico circular; jatos oscilantes; e interação fluido-estrutura. A seguir, são apresentados os detalhes das simulações.

\subsection{Colapso de um Bloco de Fluido}

Este escoamento de um colapso de um bloco de fluido sob à ação da gravidade constituem uma versão 3D do problema 2D descrito na seção 6.1.3. Desta forma, nesta seção, pretende-se validar o código "Freeflow-3D" equipado com as novas estratégias "upwind" denominadas de ALUS e TOPUS. Os resultados descritos abaixo foram parcialmente publicados em anais de eventos e periódico (ver referências [48, 112, 113]).

Para a simulação do problema, considera-se um bloco de fluido em repouso com dimensões $0.05 \mathrm{~m} \times 0.1 \mathrm{~m} \times 0.1 \mathrm{~m}$. As escalas envolvidas no problema são $L=0.05 \mathrm{~m}$ e $U_{0}=\sqrt{0.4905} \mathrm{~m} / \mathrm{s}$, de maneira que o número de Reynolds é $R e=9.91 \times 10^{4}$. Uma malha de $150 \times 50 \times 75(\delta x=$ $\delta y=\delta z=0.002 m$ ) células computacionais foi usada. A Figura 7.1 mostra a solução numérica do colapso, em quatro tempos, obtida com o esquema TOPUS, onde pode se ver a evolução do escoamento como um todo. As distribuições de pressão obtidas com os esquemas ALUS e TOPUS são mostradas na Figura 7.2 (caso (a) - ALUS e caso (b) - TOPUS).

A Figura 7.3 mostra o confronto entre os dados numéricos obtidos com o ADBQUICKEST, ALUS, CUBISTA e TOPUS, e os dados experimentais de Koshizuka/Oka e Martin/Moyce [70, 87] para a frente do fluido $\left(x_{\max }\right)$ em função do tempo. As soluções numéricas são extraídas no plano de corte $y=0.05 \mathrm{~m}$. De maneira geral, observa-se nessa figura a boa concordância entre os dados numéricos e os dados experimentais. 


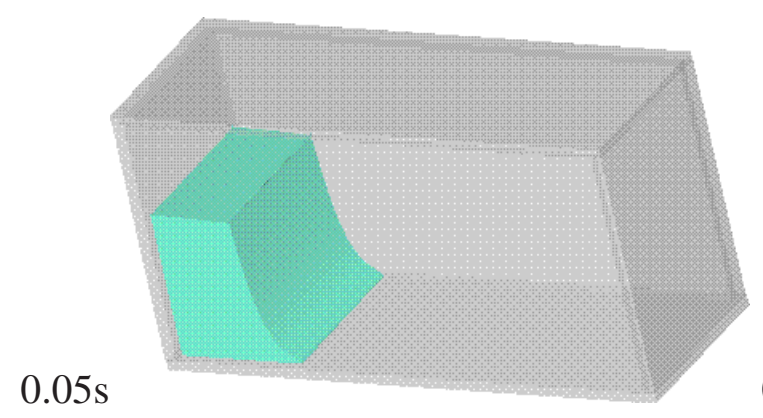

$0.15 \mathrm{~s}$
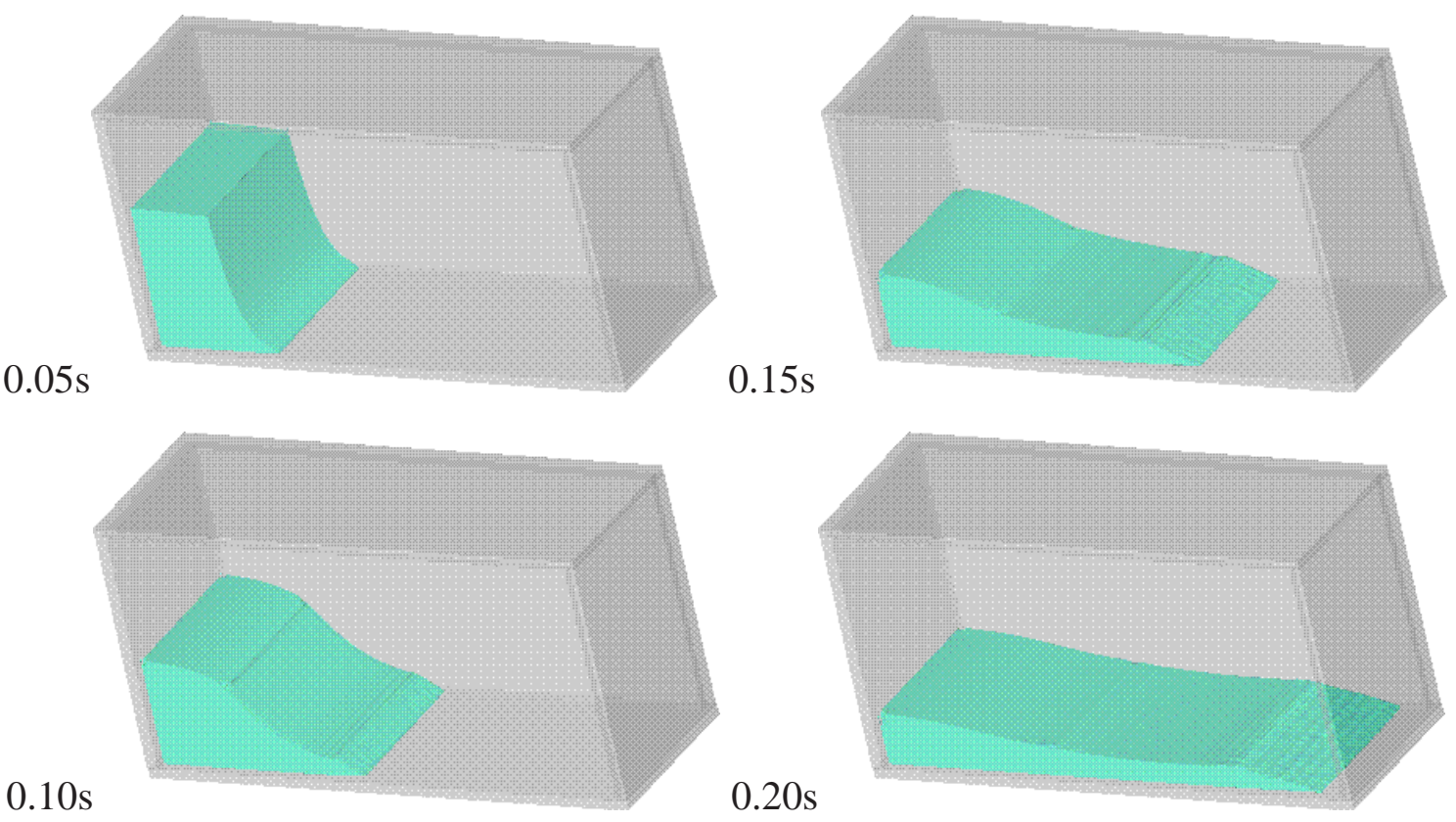

Figura 7.1: Solução numérica do colapso de um bloco de fluido sob à ação da gravidade utilizando o esquema TOPUS.

\subsection{Ressalto Hidráulico Circular}

Nesta seção são apresentados resultados numéricos para o ressalto hidráulico circular obtidos com os esquemas ALUS e TOPUS incrementado no ambiente de simulação "Freeflow-3D" [20]. Esses resultados foram publicados em anais de eventos (ver [105, 112]). Ressaltos hidráulicos são problemas que aparecem em aplicações tecnológicas (ver, por exemplo, [12]) e podem ser observados no movimento de líquidos em camadas finas (películas) sobre superfícies sólidas. Nesses problemas estão presentes o transporte de energia, a transferência de massa e, sobretudo, em analogia com as ondas de choque, a presença de uma descontinuidade no movimento. Tais peculiaridades mais a presença da superfície livre móvel criam sérias dificuldades no tratamento numérico desses problemas. O conhecimento do campo de velocidade em várias regiões do escoamento é indispensável para o bom entendimento dos fenômenos envolvidos.

Escoamentos radiais em camadas finas formam uma classe especial de películas que podem ser observadas quando jatos circulares de fluido incidem sobre superfícies sólidas planas e impermeáveis. Nessas condições, os jatos espalham radialmente pelas superfícies a partir do ponto de estagnação. Os efeitos de inércia, gravidade, viscosidade e tensão superficial governam tais problemas. Segundo a literatura (veja, por exemplo, $[19,73,141]$ ) durante o espalhamento a espessura de fluido decresce e, então, a uma certa distância cresce repentinamente sob à ação de um gradiente de pressão adverso. Esse gradiente pode causar separação da película em alguma distância radial, levando à formação de um fenômeno interessante conhecido como ressalto hidráulico circular. Hoje em dia, as várias formas do ressalto hidráulico constituem excelentes problemas com superfícies livres para validar métodos numéricos. O desafio maior tem sido simular numericamente as estruturas poligonais de Ellegard e seus colaboradores [38,39]. Por exemplo, no trabalho de Ferreira et 
(a) ALUS
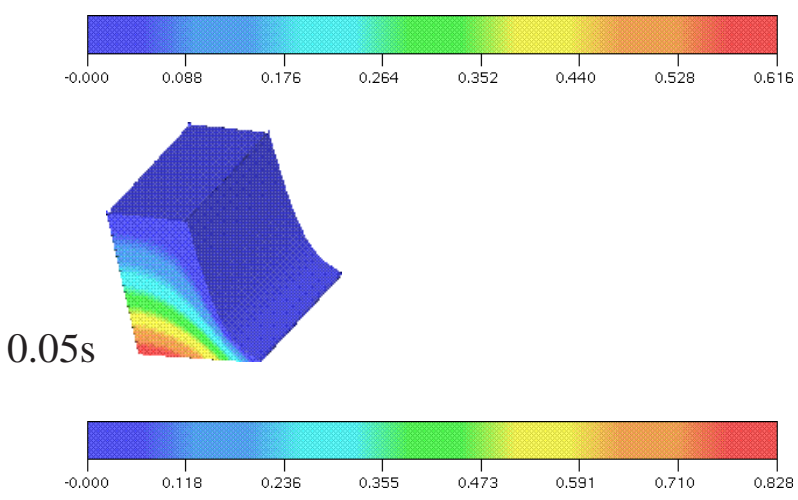

$0.1 \mathrm{~s}$
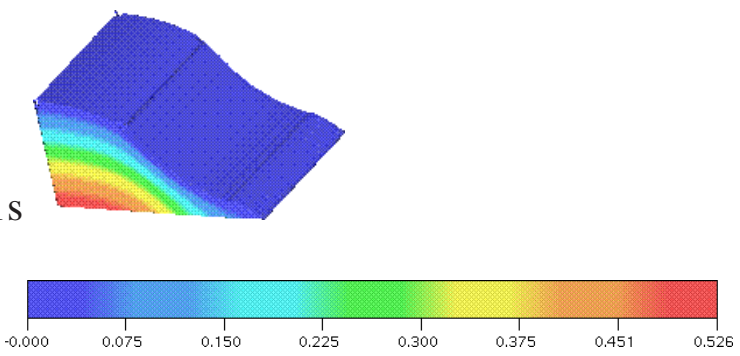

$0.15 \mathrm{~s}$
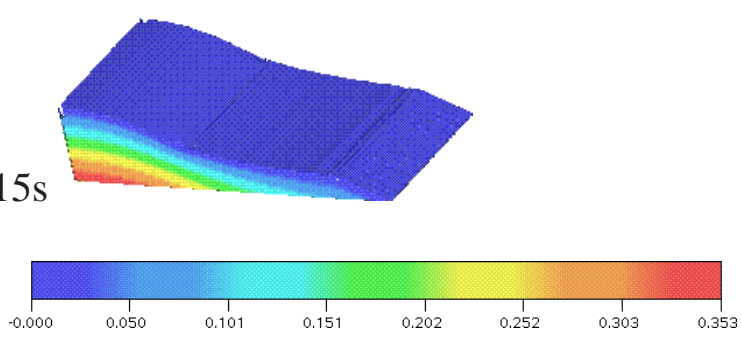

$0.20 \mathrm{~s}$

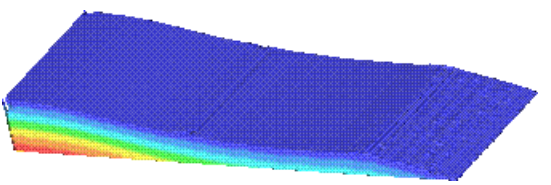

(b) TOPUS

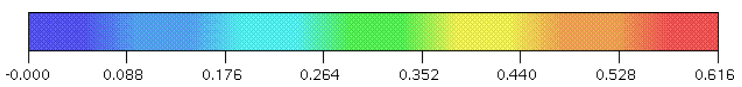

$0.05 \mathrm{~s}$

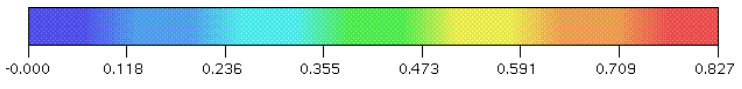

$0.10 \mathrm{~s}$

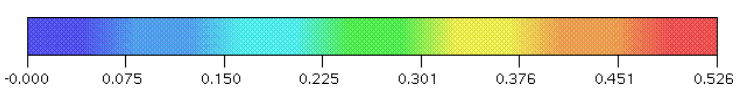

\section{$0.15 \mathrm{~s}$}
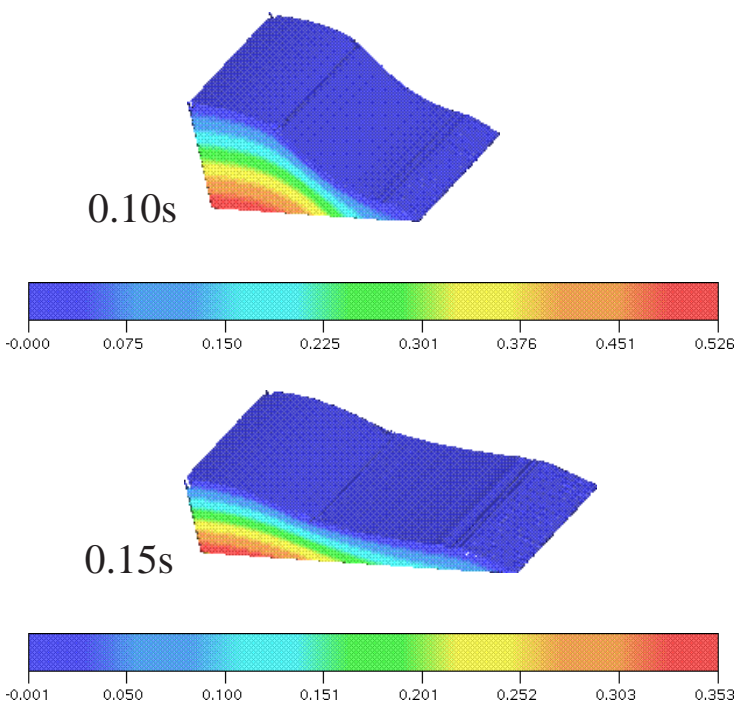

$0.20 \mathrm{~s}$

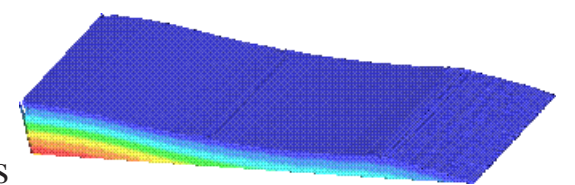

Figura 7.2: Distribuição da pressão calculada na simulação do colapso de um bloco de fluido usando os esquemas ALUS e TOPUS. 
(a) ADBQUICKEST

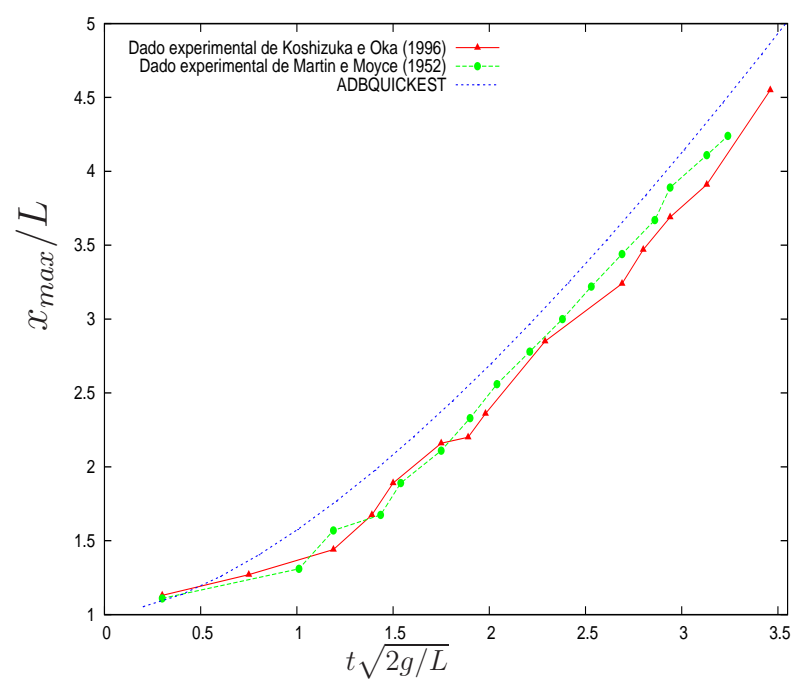

(c) CUBISTA

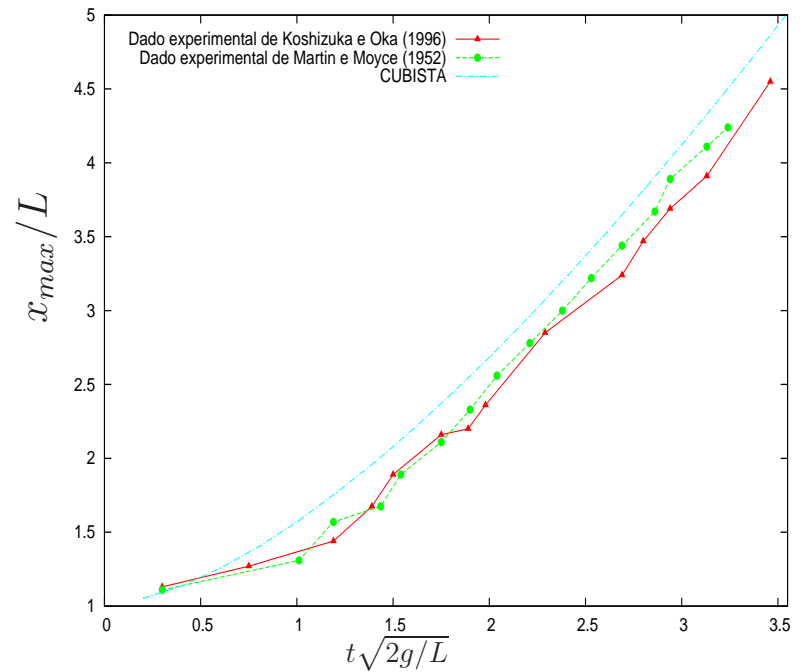

(b) ALUS

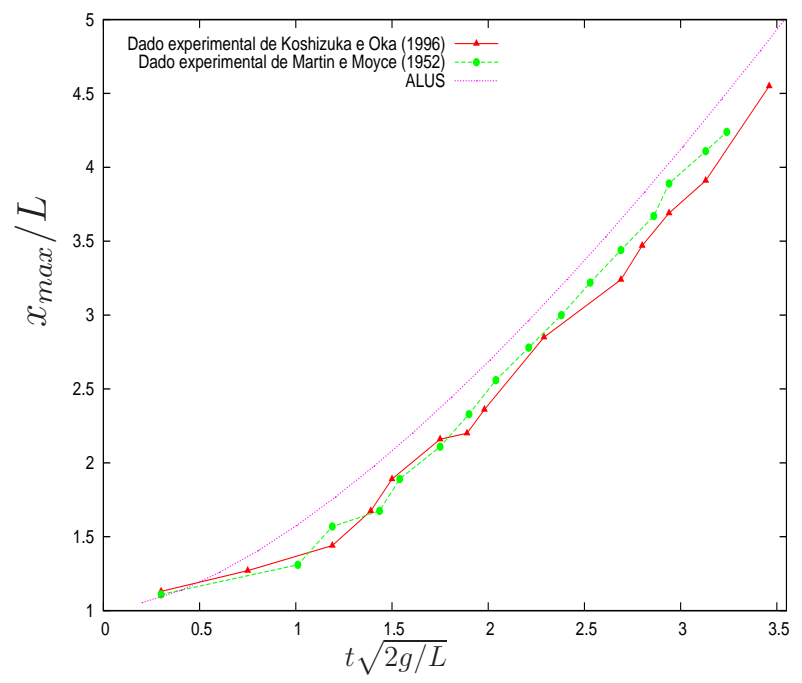

(d) TOPUS

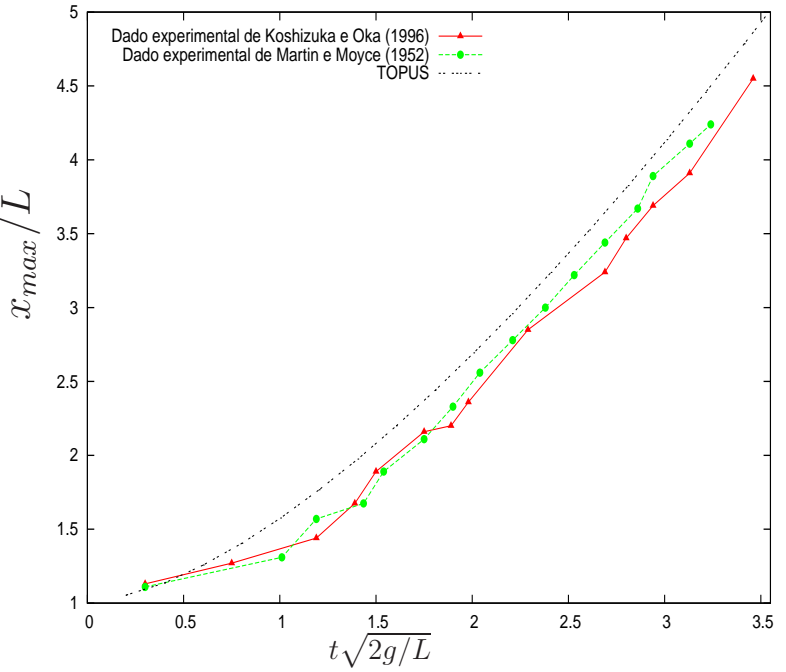

Figura 7.3: Comparação entre a frente principal $x_{\max }$ do fluido, em função do tempo, calculada pelas soluções numéricas e experimentais do colapso de um bloco de fluido. 
al. [45] fez-se uma tentativa de simular o fenômeno com o código "Freeflow-3D" equipado com o esquema de primeira ordem FOU, cujo resultado está apresentado na Figura 7.4. Vê-se claramente neste resultado que, por causa da dissipação numérica, o fenômeno não foi simulado corretamente, isto é, ao invés de produzir um salto hidráulico circular, o código forneceu um salto hidráulico "quadrado".

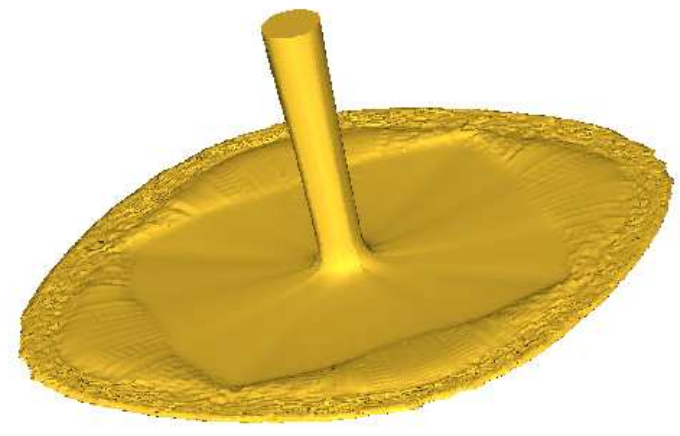

Figura 7.4: Resultado numérico [45] fornecido pelo código "Freeflow-3D" equipado o esquema "upwind" de primeira ordem.

Com o objetivo de mostrar que o código "Freeflow-3D" atual, isto é, equipado com os esquemas ALUS e TOPUS, de fato simula o ressalto hidráulico circular, apresentam-se a seguir dois casos teste:

- Caso 1 - Simulação do ressalto hidráulico circular à Reynolds $R e=150$ : neste caso, os seguintes dados foram empregados: dimensão do domínio computacional $0.1 \mathrm{~m} \times 0.1 \mathrm{~m} \times$ $\times 0.02 \mathrm{~m}$, diâmetro do injetor $0.01 \mathrm{~m}$ e altura do injetor $0.002 \mathrm{~m}$, e velocidade de injeção $0.5 \mathrm{~m} / \mathrm{s}$. Na simulação desse caso são adotados uma malha de $100 \times 100 \times 20(\delta x=$ $\delta y=\delta z=0.001 \mathrm{~m}$ ) células computacionais e tempo final de simulação $t=0.5 \mathrm{~s}$. O resultado numérico obtido na simulação do problema com o TOPUS é apresentado na Figura 7.5b. Nota-se dessa figura que o método numérico capturou o fenômeno físico presente no escoamento, como ilustrado na Figura 7.5a. A simulação desse caso com o esquema ALUS forneceu resultados bem próximos daqueles obtidos com o esquema TOPUS (não mostrados).

- Caso 2 - Simulação do ressalto hidráulico circular à Reynolds $R e=1.0 \times 10^{3}$ : aqui, os seguintes dados foram empregados: dimensão do domínio computacional $0.6 m \times 0.6 m \times$ $\times 0.05 \mathrm{~m}$, diâmetro do injetor $0.05 \mathrm{~m}$, altura do injetor $0.001 \mathrm{~m}$, e velocidade de injeção $1.0 \mathrm{~m} / \mathrm{s}$. Na simulação desse caso são adotados uma malha de $120 \times 120 \times 10(\delta x=\delta y=$ $\delta z=0.005 \mathrm{~m}$ ) células computacionais e tempo final de simulação $t=1.5 \mathrm{~s}$. O resultado numérico obtido na simulação do problema com o TOPUS é apresentado na Figura 7.6b. Nota-se neste caso que o método numérico capturou também o fenômeno físico, como está 
ilustrado na Figura 7.6a. A simulação desse caso com o esquema ALUS forneceu também resultados bem próximos daqueles obtidos como o esquema TOPUS (omitidos).

(a) Dado experimental [90]

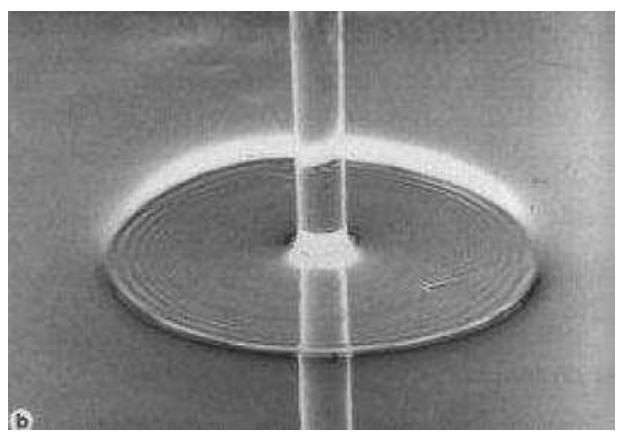

(b) Resultado numérico

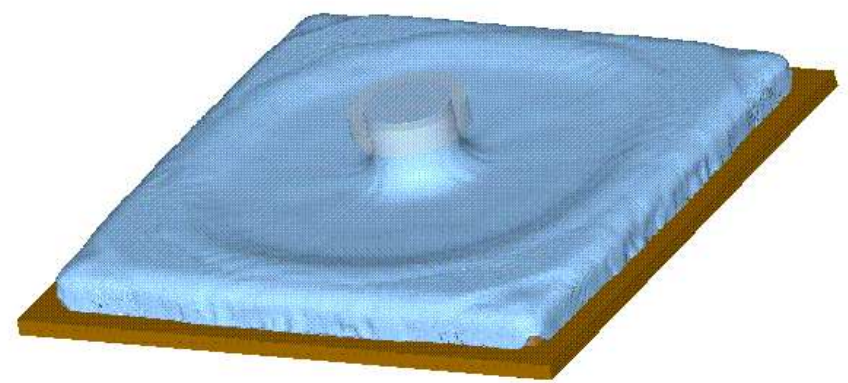

Figura 7.5: Comparação qualitativa entre os resultados numérico e experimental do ressalto hidráulico circular, $R e=150$.

(a) Dado experimental [37]

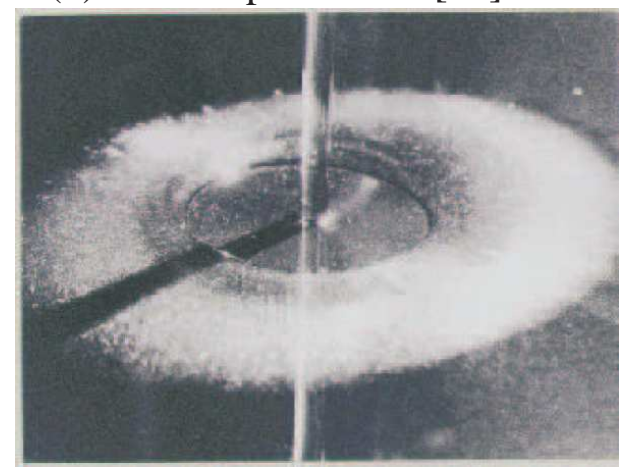

(b) Resultado numérico

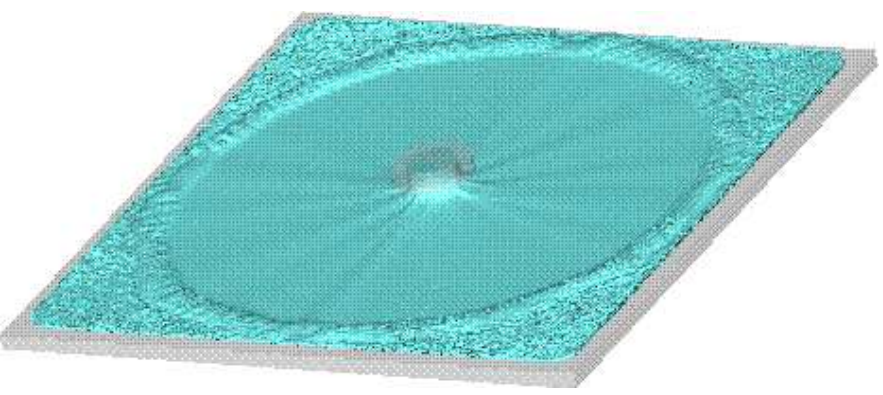

Figura 7.6: Comparação qualitativa entre os resultados numérico e experimental do ressalto hidráulico circular em regime de transição para a turbulência, $R e=1.0 \times 10^{3}$. 


\subsection{Jatos Oscilantes}

Soluções numéricas das instabilidades físicas em jatos oscilantes são apresentadas nesta seção. Estes dados numéricos foram publicados em anais de eventos (ver [105]). Instabilidades físicas em jatos de fluido a baixos Reynolds podem ser observadas facilmente no cotidiano. Por exemplo, o mel de abelha escorrendo de uma colher, ou ainda o creme condicionador caindo na mão no banho diário. Esses problemas tornam-se instáveis quando o injetor situa-se a certa distância de uma superfície sólida. Sob certas condições específicas, como foi mostrado experimentalmente por Cruickshank [27], alguns fluidos com alto coeficiente de viscosidade afinam devido ao efeito da tensão superficial e à presença do campo gravitacional, e ao aderirem a superfícies rígidas formam dobras ou espirais, e, por essa razão, são denominados de jatos oscilantes. Taylor [142] foi talvez um dos pioneiros a estudar tal fenômeno.

Para a simulação das instabilidades em jatos oscilantes, os seguintes dados foram empregados: dimensão do domínio computacional $0.1 m \times 0.1 m \times 0.1 m$; diâmetro do injetor $0.01 \mathrm{~m}$; altura do injetor $0.09 \mathrm{~m}$; e velocidade de injeção $1.0 \mathrm{~m} / \mathrm{s}$. Nas simulações são utilizados os números de Reynolds $R e=0.25$ e $R e=0.50$ e uma malha de $100 \times 100 \times 100(\delta x=\delta y=\delta z=0.001 \mathrm{~m})$ células computacionais. O tempo de simulação considerado é $1.5 \mathrm{~s}$. Como pode ser observado na Figura 7.7, as instabilidades físicas foram capturadas com sucesso para os jatos circular a Reynolds 0.25 e planar a Reynolds 0.5 . Mais uma vez, isto mostra que o esquema TOPUS, embora proposto para simular problemas a altos Reynolds, pode ser utilizado também, com sucesso, na simulação de escoamentos a baixos Reynolds. A simulação desse problema com o esquema ALUS forneceu resultados bem próximos daqueles obtidos como o esquema TOPUS, os quais foram omitidos.

(a) Jato circular $-R e=0.25$

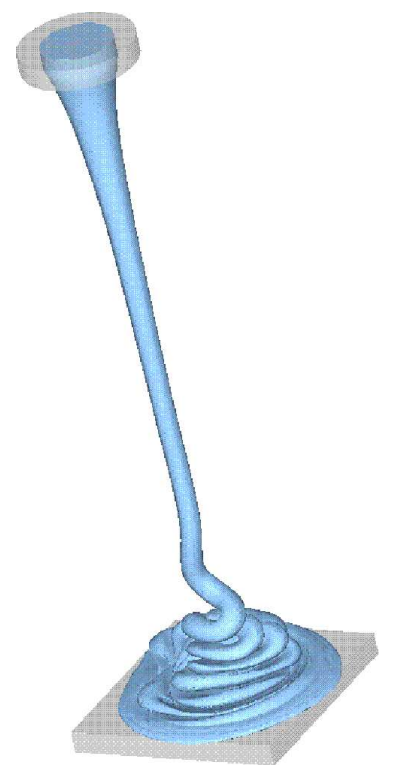

(b) Jato planar $-R e=0.50$

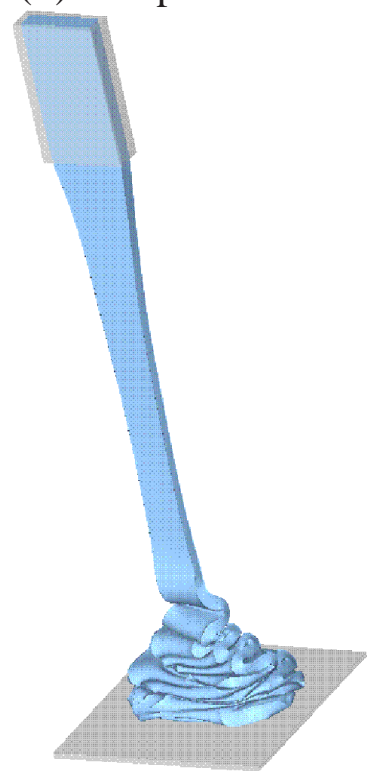

Figura 7.7: Soluções numéricas para escoamentos de jatos oscilantes. 


\subsection{Interação Fluido-Estrutura}

Apresenta-se aqui a solução numérica [105] obtida na simulação de um problema envolvendo a interação fluido - estrutura. Nesse problema o esquema TOPUS foi utilizado. A interação fluido-estrutura é um tópico importante em muitos campos da engenharia hidráulica. Exemplos incluem o estudo de ondas contra estruturas (ver [31, 52]), e a simulação de quebra de ondas por Löhner et al. [86]. Uma situação bastante simplificada de fluido-estrutura, e útil para validar métodos numéricos, é o efeito combinado da superfície livre do fluido e sua viscosidade, tal como o choque de uma onda de superfície contra um obstáculo (ver $[59,84])$. Este problema foi simulado a Reynolds $R e \approx 5.15 \times 10^{5}$ numa malha de $170 \times 70 \times 70(\delta x=\delta y=\delta z=0.010 \mathrm{~m})$ células computacionais. A altura do obstáculo é $H=0.5 \mathrm{~m}$, e está situado a distância de $1 \mathrm{~m}$ de uma das faces da caixa. A Figura 7.8 mostra a interação de uma onda e um obstáculo após $t=1.5 \mathrm{~s}$. Como se pode ver por meio desta figura, o esquema TOPUS mostrou ser útil também em problemas envolvendo a interação fluido-estrutura. O esquema ALUS forneceu, praticamente, o mesmo resultado como o apresentado pelo TOPUS.

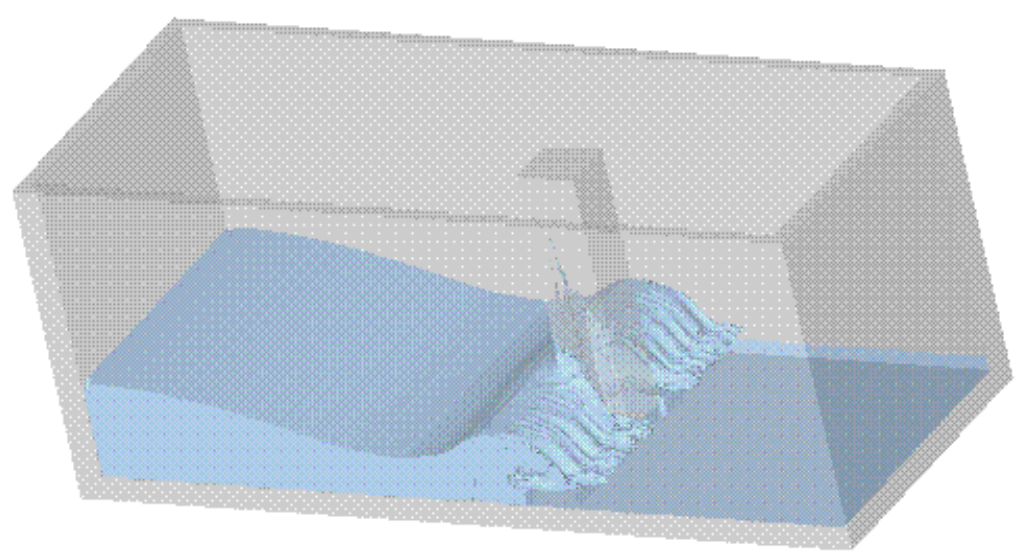

Figura 7.8: Solução numérica do choque de uma onda contra um obstáculo rígido. 


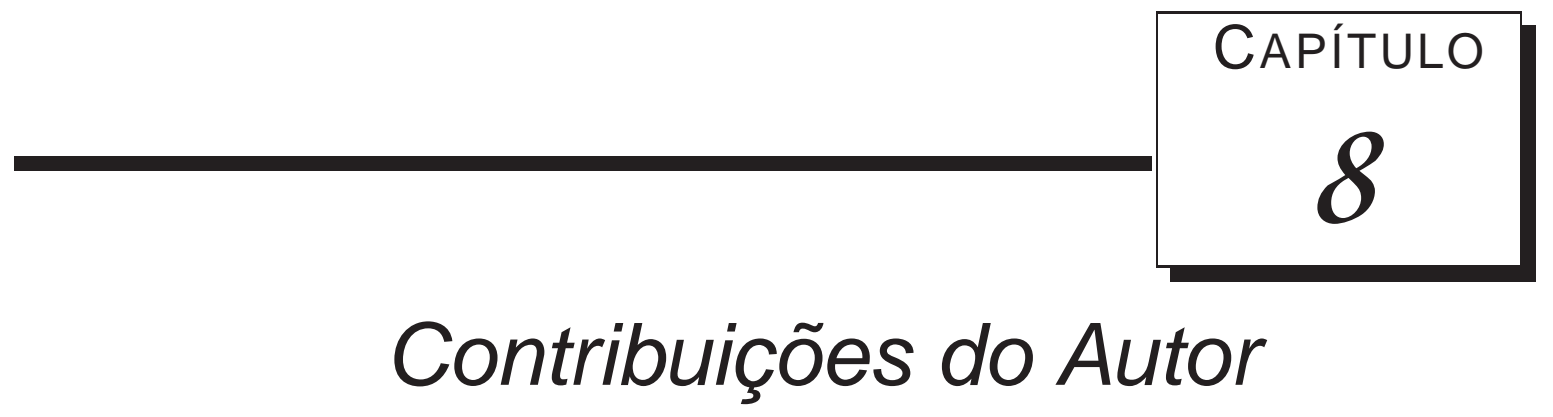

Neste capítulo são apresentados os principais trabalhos produzidos pelo autor e seus colaboradores durante esta pesquisa.

\subsection{Artigos Publicados e Submetidos em Periódicos}

Resumo do artigo: "An assessment of a high-order finite difference upwind scheme for the simulation of convection-diffusion problems" [48]

O artigo apresenta o estudo do desenvolvimento e aplicação do esquema QUICKEST adaptativo (chamado doravante ADBQUICKEST), uma versão do esquema QUICKEST de Leonard para problemas não-estacionários, empregando discretização dos termos convectivos lineares e não lineares. O esquema é aplicado numa ampla gama de problemas em dinâmica dos fluidos computacional, onde os fenômenos de transporte são de interesse especial. Em particular, o desempenho do esquema é investigado por meio de um estudo extensivo de simulações numéricas de problemas envolvendo advecção-difusão. O esquema, implementado no contexto da metodologia das diferenças finitas, combina uma boa resolução de choques (ou descontinuidades) com uma boa aproximação de partes suaves das soluções. Para averiguar o desempenho do esquema, sete problemas testes foram considerados, a saber: (i) advecção de escalares; (ii) equação de Burgers não linear; (iii) equações de Euler da dinâmica dos gases; (iv) escoamento newtoniano num canal; (v) jato newtoniano com simetria radial; (vi) escoamento não newtoniano num duto; e (vii) colapso de fluido sob à ação do campo gravitacional. Os experimentos numéricos mostram claramente que o esquema ADBQUICKEST fornece resultados mais consistentes que aqueles encontrados na literatura. A partir deste estudo, a flexibilidade e robustez desse esquema "upwind" de alta resolução são confirmadas por demonstrando sua capacidade em resolver uma variedade de problemas lineares e não lineares com ou sem soluções descontínuas. 
Resumo do artigo: "An upwind differencing scheme for conservation laws and related fluid dynamics problems" [50]

$\mathrm{O}$ artigo mostra o desenvolvimento do esquema TOPUS, no contexto NVD/CBC/TVD e diferenças finitas, para discretização dos termos convectivos lineares e não lineares presentes em leis de conservação e problemas relacionados em dinâmica dos fluidos. Para averiguar o desempenho do esquema, oito problemas testes foram considerados, a saber: (i) advecção de escalares; (ii) equação de Burgers com e sem viscosidade; (iii) equações de Euler da dinâmica dos gases; (iv) escoamento newtoniano num canal; (v) jato newtoniano com simetria radial; (vi) colapso de fluido sob à ação do campo gravitacional; (vii) ressalto hidráulico circular; (viii) escoamento compressível ao redor de aerofólios. Os experimentos numéricos mostram claramente que o esquema TOPUS fornece resultados mais consistentes que aqueles encontrados na literatura. Fica evidente a partir deste estudo que esse novo esquema polinomial "upwind" de alta resolução é bastante robusto para resolver uma variedade de problemas lineares e não lineares com ou sem choques.

Resumo do artigo: "Uma avaliação computacional de esquemas de alta resolução em problemas advectivos-difusivos" [102]

No presente trabalho é apresentada uma avaliação computacional dos esquemas de alta resolução WACEB, CUBISTA e QUICKEST adaptativo em resolver problemas lineares e não lineares de advecção-difusão. Utilizando-se a metodologia de diferenças finitas, esses esquemas são analisados e implementados no contexto de variáveis normalizadas de Leonard. Para acessar o desempenho dos esquemas, dois problemas testes são considerados, a saber: advecção de um escalar; e o problema de Riemann para as equações de Euler 1D. Os resultados numéricos obtidos nesses testes mostram que os três esquemas funcionam bem, com o esquema QUICKEST adaptativo fornecendo os melhores resultados no caso não linear. Como aplicação, o esquema QUICKEST adaptativo é utilizado na solução numérica das equações de Burgers 1D com viscosidade e de Navier-Stokes 3D (problemas do ressalto hidráulico e jatos oscilantes).

Resumo do artigo: "A high resolution CBC/TVD upwind scheme for unsteady flows with shock waves" [104]

$\mathrm{O}$ artigo apresenta o esquema de alta resolução CBC/TVD denominado TOPUS. Aplicações em problemas envolvendo leis de conservações gerais são realizadas para acessar o desempenho desse esquema. Em particular, a equação de Buckley-Leverett e o escoamento supersônico ao redor do aerofólio NACA 0012 são adotados. Os resultados numéricos confirmam a robustez do TOPUS na obtenção de solução com descontinuidades.

Resumo do artigo: "A new high resolution TVD scheme for unsteady flows with shock waves" [109]

O artigo apresenta a formulação matemática de um novo esquema TVD de alta resolução denominado TOPUS. Aplicações em problemas envolvendo escoamentos transientes com descontinuidades modelados pelas equações de Euler e de Burgers são realizadas para aferir seu desempenho. Em particular, tubos de choque 1D (Sod, Shu-Osher, Toro), Burgers 1D sem viscosidade, escoamento supersônico ao redor do aerofólio diamante e escoamento transônico ao redor do aerofólio RAE 2822 foram as aplicações realizadas. Os resultados obtidos para os problemas compressíveis usando o TOPUS mostram boa concordância com dados experimentais. 


\subsection{Artigos Publicados em Anais de Eventos}

Resumo do artigo: "Evaluation of a new TVD limiter for transonic and supersonic flows" [30]

Este artigo trata da avaliação do limitador de fluxo derivado do esquema TOPUS em simulações de escoamentos transônicos e supersônicos. Os resultados obtidos com este limitador TVD foram comparados com dados experimentais e soluções numéricas fornecidas pelo esquema van Albada. Esses resultados mostram que o TOPUS consegue capturar o choque livre de oscilações e é bastante apropriado para as simulações de escoamentos transônicos e supersônicos ao redor de aerofólios.

Resumo do artigo: "The performance of a high order upwind scheme for the numerical simulation of fluid flow problems" [75]

O objetivo deste artigo é apresentar o desempenho do esquema QUICKEST adaptativo em simulações numéricas das equações de Euler e de Navier-Stokes 2D. A metodologia de diferenças finitas é usada na discretização das EDPs. Os resultados numéricos obtidos com o esquema QUICKEST adaptativo foram comparados com aqueles obtidos com os esquemas SMART e Superbee. O QUICKEST adaptativo foi o esquema que teve o melhor desempenho nos experimentos numéricos realizados.

Resumo do artigo: "Esquemas polinomiais upwind e suas aplicações em escoamentos incompressíveis 3D transientes" [105]

Este artigo apresenta a aplicação dos esquemas "upwind" de terceira ordem (TOPUS com $\alpha=$ 0 e $\alpha=2$ ) em simulações de escoamentos incompressíveis 3D (jatos oscilantes, salto hidráulico, interação fluido-estrutura). Para testar os esquemas, neste estudo, foram consideradas as equações de advecção de um escalar e o problema de Riemann para as equações de Euler 1D. De modo geral, nas verificações 1D ambos os esquemas forneceram soluções numéricas bastante satisfatórias e sem oscilações. Os resultados numéricos nas aplicações 3D indicam que os fenômenos físicos a baixos e a altos Reynolds podem ser simulados com confiança.

Resumo do artigo: "Numerical simulation of turbulent free surface flows using a combination of a new high order upwind scheme and a realizable Reynolds stress algebraic model" [108]

Este artigo apresenta a aplicação do TOPUS, um novo esquema "upwind” de alta resolução, em simulação de escoamentos turbulentos 2D com superfícies livres. Para estimar o tensor de tensões de Reynolds, um modelo algébrico não linear foi implementado. A discretização das equações médias de Navier-Stokes é realizada com a metodologia de diferenças finitas numa malha deslocada. A verificação e a validação do método numérico são realizadas considerando, respectivamente, os escoamentos turbulentos de um jato incidindo numa superfície rígida e o colapso de um bloco de fluido 2D. Como aplicação 2D, os problemas "sluice gate" e de um jato penetrando num fluido em repouso são simulados. A combinação do esquema TOPUS com o modelo não linear, respectivamente, para estimar o termo convectivo e tensor de tensões de Reynolds é bastante robusta para simular escoamentos turbulentos.

Resumo do artigo: "Desenvolvimento e aplicação de esquemas upwind de terceira ordem para transporte convectivo" [110]

Este artigo apresenta a investigação de dois esquemas "upwind" de terceira ordem (TOPUS com $\alpha=0$ e $\alpha=2$ ) em simulações de problemas transientes lineares (advecção) e não lineares (tubo de choque). Os resultados numéricos obtidos são comparados com soluções exatas e de 
referência, mostrando concordância bastante razoável entre si. Os dois esquemas tiveram desempenhos similares nos testes.

Resumo do artigo: "Direct computation of incompressible turbulent free surface flow using a new high order upwind scheme" [112]

Este artigo apresenta a aplicação do TOPUS em simulação direta (sem modelagem de turbulência) de escoamentos turbulentos 2D/3D com superfícies livres. A discretização das equações de Navier-Stokes é realizada com a metodologia de diferenças finitas numa malha deslocada. O método numérico utilizado é uma adaptação da metodologia SMAC ("Simplified Marker-And-Cell”) para simular escoamentos com superfícies livres a alto número de Reynolds. O método numérico combinado com o TOPUS é verificado e validado, respectivamente, nos problemas de um jato 2D incidindo numa superfície impermeável e do colapso de um bloco de fluido 2D/3D. Como aplicação, três problemas são adotados: jato 2D penetrando num fluido em repouso, "sluice gate" 2D e salto hidráulico 3D. Os resultados obtidos nos testes mostram que o TOPUS forneceu soluções confiáveis quando comparadas com dados analíticos e experimentais. As soluções numéricas obtidas nas aplicações são bastante consistentes com o fenômeno físico dos problemas simulados.

Resumo do artigo: "New high order upwind techniques for advective term discretizations" [113]

Este artigo apresenta os esquemas ALUS e TOPUS para discretização de termos convectivos das equações de Navier-Stokes. Para teste desses esquemas, a equação de Burgers 1D sem viscosidade é adotada. Como aplicação destas novas estratégias "upwind", o problema confinado com expansão brusca 2D (regime laminar) e o jato incidindo numa superfície rígida são simulados. Os resultados numéricos do ALUS e TOPUS são comparados com soluções analíticas e experimentais. A capacidade desses esquemas "upwind" para resolver os problemas modelados pelas equações de Navier-Stokes 2D/3D e de Burgers inviscida 1D é confirmada neste estudo.

Resumo do artigo: "A polynomial upwind scheme for convection discretization" [114]

O objetivo deste artigo é avaliar o esquema TOPUS em simulações de problemas 1D lineares (camada limite) e não lineares (Burgers com viscosidade, tubo de choque de Toro) dominadas por convecção. Os resultados fornecidos durante a simulação do TOPUS são bastante consistentes com soluções analíticas e demonstram que no mínimo segunda ordem de exatidão é alcançada para os problemas adotados neste estudo.

Resumo do artigo: "Development and implementation of polynomial scheme for the numerical solution of $1 D$ conservation laws" [115]

$\mathrm{O}$ artigo apresenta o desenvolvimento e a implementação do esquema TOPUS, no contexto de diferenças finitas e NVD/CBC/TVD, para tratamento dos termos convectivos em leis de conservação 1D. Esta nova estratégia polinomial "upwind" foi testada em problemas 1D modelados pelas equações de Euler e de Navier-Stokes. Comparações com os esquemas ADBQUICKEST, CUBISTA, SMARTER, Superbee, WACEB foram consideradas neste estudo. Os resultados fornecidos na simulação usando o esquema TOPUS são confiáveis quando comparados com as soluções analíticas e de referências. 


\section{Conclusões e trabalhos futuros}

Neste trabalho de mestrado foram apresentados os resultados do desenvolvimento e teste dos esquemas "upwind" de alta resolução ALUS e TOPUS. O propósito foi controlar a difusão numérica em leis de conservação 1D/2D e escoamentos de fluidos transientes $2 \mathrm{D} / 3 \mathrm{D}$. O desempenho, a validação e a verificação desses novos esquemas foram analisados com respeito a vários problemas teste, a saber: advecção de escalares 1D; problemas de convecção-difusão 1D; problemas de Riemann para a equação de Euler, Burgers e águas rasas 1D; e escoamentos transônicos/supersônicos $2 \mathrm{D}$ ao redor de aerofólios. A partir dos resultados $1 \mathrm{D}$, pode-se concluir que os esquemas ALUS e TOPUS são estratégias "upwind" robustas para capturar descontinuidades, quando comparadas com soluções analíticas e de referências. Destaca-se dos resultados obtidos para advecção de escalares e transporte pelas equações de Burgers e Euler que os esquemas ALUS e TOPUS aqui propostos são qualitativamente superiores que a muitos esquemas convectivos consagrados na literatura. Para escoamentos sobre aerofólios, o esquema TOPUS forneceu resultados compatíveis com aqueles de van Albada [150] (um esquema de referência) e dados experimentais. Como aplicações os esquemas ALUS e TOPUS foram usados na simulação de escoamentos 2D e 3D laminares e turbulentos com superfícies livres móveis numa ampla faixa do número de Reynolds, cujos resultados demonstraram que eles constituem ferramentas efetivas para estudar escoamentos complexos com superfícies livres. Em particular, para a simulação do fenômeno da turbulência, uma modelagem algébrica não linear para o tensor de Reynolds foi analisada e implementada no sistema de simulação "Freeflow-2D".

Em resumo, os resultados apresentados neste trabalho fornecem uma gama bastante significativa de dados (mais precisos) de simulações numéricas utilizando-se os dois novos esquemas CBC/TVD: ALUS e TOPUS. Isto permitirá no futuro pesquisadores comparar seus dados com os aqui apresentados.

A pesquisa científica descrita neste texto pode ser incrementada de várias maneiras, sobretudo no que diz respeito à combinação de esquemas de diferenças "upwind" de alta resolução e modelagem da turbulência $\kappa-\varepsilon$ com aproximação não linear para o tensor de tensões de Reynolds. Os aspectos mais relevantes dos temas que serão considerados na pesquisa futura incluem:

- Os esquemas ALUS e TOPUS podem ser aplicados também numas variedades de problemas de escoamentos incompressíveis com superfícies livres móveis. Como exemplos, pode-se citar os problemas envolvendo tensão superficial, transferência de calor, fenômenos de convecção e difusão que ocorrem nos fluidos não-newtonianos com efeitos de elasticidade e os problemas multifásicos (em particular gás - sólido);

- O problema fundamental da precisão dos esquemas apresentados aqui em escoamentos 3D realísticos é um assunto importante que precisa ser bem explorado. Para tanto, a simulação das 
diferentes estruturas poligonais do salto hidráulico circular de Ellegard e colaboradores (ver [38, 39]) constitui um excelente exemplo de validação;

- A captura correta do choque, isto é a ausência de pontos soluções no choque, é um tema atual e merece ser investigado no contexto da pesquisa aqui descrita. Para tanto, pretende-se associar os esquemas ALUS e TOPUS com a técnica RCM ("Random Choice Method") de Glimm [56] (ver também aplicação em [162]);

- A combinação dos esquemas ALUS e TOPUS e modelagem algébrica do tensor de tensões de Reynolds para a simulação de escoamentos turbulentos com superfícies livres é outra proposta interessante de pesquisa para o futuro. Em relação à modelagem do tensor de Reynolds, neste trabalho foi implementado o modelo algébrico de Shih et al. [123], pretende-se no próximo passo implementar e aplicar os modelos não lineares de Gatski e Speziale [54] e de Girimaji [55] em simulação de escoamentos com e sem superfícies livres;

- Um novo limitador de fluxo flexível e simétrico baseado no esquema TOPUS, cujo desenvolvimento é apresentado no apêndice B, será contemplado nas próximas aplicações. Destaca-se que a simetria é uma propriedade muito desejável [10] que afeta positivamente a resolução do choque;

- A modelagem matemática dos esquemas ALUS e TOPUS no contexto NVSF ("Normalized Variable and Space Formulation") de Darwish e Moukalled [33] é um assunto de grande interesse na comunidade científica em CFD, a qual merece investigação (ver a formulação já desenvolvida no apêndice $\mathrm{C}$ );

- Implementação dos esquemas de alta resolução (ALUS, FSFL e TOPUS) desenvolvidos neste trabalho de mestrado em outros ambientes computacionais de CFD, por exemplo, nos códigos abertos MFIX ("Multiphase Flow with Interphase eXchanges") [89] e OpenFOAM ("Open Field Operation and Manipulation") [97, 107].

- Comparação do desempenho do ALUS e TOPUS com outros esquemas de alta resolução no tocante a tempo de CPU necessário para a obtenção da solução de um problema predominantemente convectivo. Devido a simplicidade matemática do ALUS e TOPUS, espera-se que eles sejam mais eficientes que os demais esquemas da literatura (VONOS, WACEB, CUBISTA e ADBQUICKEST).

- O código "Freeflow" atual emprega malhas uniformes para discretizar o domínio de solução. Um refinamento localizado da malha computacional é, então, fator essencial para uma análise mais elaborada dos métodos aqui apresentados, quando aplicados nas vizinhanças de paredes rígidas e camadas cizalhantes, pois é muito importante resolver com boa precisão a camada limite, uma vez que é nela onde os gradientes elevados aparecem. 


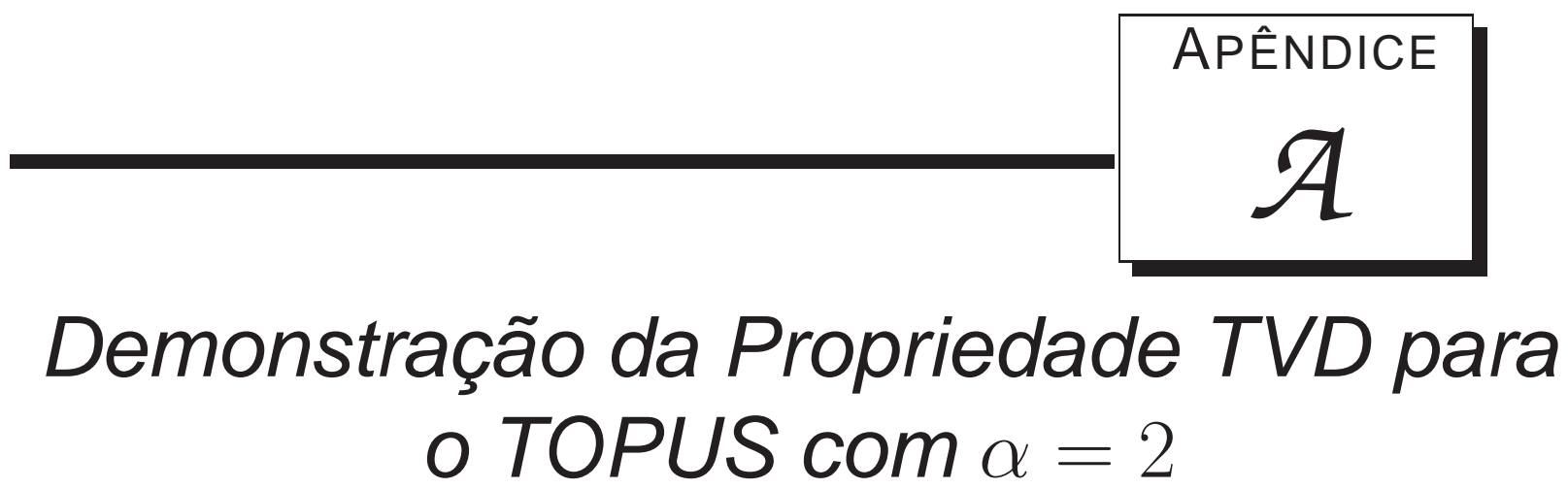

Neste apêndice apresenta-se a demonstração que o esquema TOPUS formulado em (3.9) com $\alpha=2$, isto é,

$$
\hat{\phi}_{f}=\left\{\begin{array}{l}
2 \hat{\phi}_{U}^{4}-3 \hat{\phi}_{U}^{3}+2 \hat{\phi}_{U}, \quad \hat{\phi}_{U} \in[0,1], \\
\hat{\phi}_{U}, \quad \hat{\phi}_{U} \notin[0,1]
\end{array}\right.
$$

satisfaz a propriedade TVD de Harten [61]. Para que um esquema convectivo tenha essa propriedade, no contexto NV, ele deve satisfazer as seguintes restrições (ver Figura 3.3a):

$$
\begin{cases}\hat{\phi}_{f} \in\left[\hat{\phi}_{U}, 2 \hat{\phi}_{U}\right] \quad \text { e } \quad \hat{\phi}_{f} \in(-\infty, 1], & \text { para } \hat{\phi}_{U} \in[0,1], \\ \hat{\phi}_{f}=\hat{\phi}_{U}, & \text { para } \hat{\phi}_{U} \notin[0,1] .\end{cases}
$$

Vê-se claramente de (A.1) que o esquema assume

$$
\hat{\phi}_{f}=\hat{\phi}_{U} \quad \text { para } \quad \hat{\phi}_{U} \notin[0,1] .
$$

Agora, deve-se mostrar que o esquema (A.1) satisfaz as outras 3 (três) restrições TVD:

1. $\hat{\phi}_{f} \geq \hat{\phi}_{U}$ para $0 \leq \hat{\phi}_{U} \leq 1$.

Dem. Seja $h=\hat{\phi}_{f}-\hat{\phi}_{U}$. Substituindo (A.1) em $h$ obtém-se $h=2 \hat{\phi}_{U}^{4}-3 \hat{\phi}_{U}^{3}+\hat{\phi}_{U}$. Agora, deve ser provado que $h \geq 0$ para $0 \leq \hat{\phi}_{U} \leq 1$. De fato, pois as raízes de $h$ são $-\frac{1}{2}$, 0 e 1 (multiplicidade 2), e com o estudo do sinal de $h$ conclui-se que $h \geq 0$ para $0 \leq \hat{\phi}_{U} \leq 1$; 
2. $\hat{\phi}_{f} \leq 2 \hat{\phi}_{U}$ para $0 \leq \hat{\phi}_{U} \leq 0.5$.

Dem. Seja $g=\hat{\phi}_{f}-2 \hat{\phi}_{U}$. Então $g=2 \hat{\phi}_{U}^{4}-3 \hat{\phi}_{U}^{3}$. Agora, deve ser provado que $g \leq 0$ para $0 \leq \hat{\phi}_{U} \leq 0.5$. As raízes reais de $g$ são 0 (multiplicidade 3 ) e 1.5. Segue da análise do sinal de $g$ que $g \leq 0$ para $0 \leq \hat{\phi}_{U} \leq 1.5$. Em particular, $g \leq 0$ para $0 \leq \hat{\phi}_{U} \leq 0.5$;

3. $\hat{\phi}_{f} \leq 1$ para $0.5 \leq \hat{\phi}_{U} \leq 1$.

Dem. Seja $p=\hat{\phi}_{f}-1$. Então $p=2 \hat{\phi}_{U}^{4}-3 \hat{\phi}_{U}^{3}+2 \hat{\phi}_{U}-1$. Deve ser provado que $p \leq 0$ para $0.5 \leq \hat{\phi}_{U} \leq 1$. As raízes de $p$ são $z_{1}=0.6647417705-0.4011272789 i$ ( $i$ é a unidade imaginária), $z_{2}=\bar{z}_{1}, 1$ e -0.8294835411 . Sabe-se que os polinômios trocam de sinal somente em seus zeros reais. Portanto, $p \leq 0$ para $-0.8294835411 \leq \hat{\phi}_{U} \leq 1$. Em particular, $p \leq 0$ para $0.5 \leq \hat{\phi}_{U} \leq 1$

Em suma, das demonstrações acima, conclui-se que o esquema TOPUS em (A.1) é TVD. 


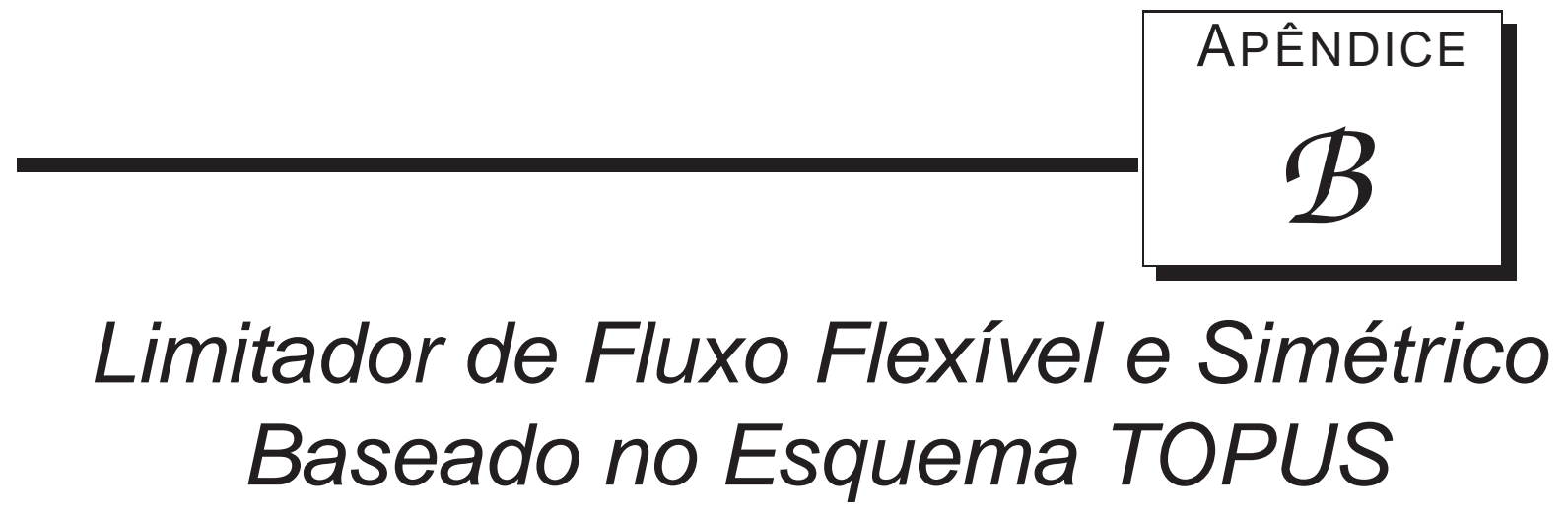

Neste apêndice é apresentado um novo limitador de fluxo flexível e simétrico baseado no esquema TOPUS, tal limitador nomeado por FSFL ("Flexible and Symmetric Flux Limiter") é mais uma contribuição deste trabalho de mestrado. A justificativa para desenvolvê-lo é o fato do esquema TOPUS não apresentar esta desejável propriedade de simetria [10], que matematicamente corresponde a

$$
\frac{\psi\left(r_{f}\right)}{r_{f}}=\psi\left(\frac{1}{r_{f}}\right)
$$

Baseando-se no limitador TOPUS em (3.17), a formulação geral do FSFL é dada por

$$
\psi\left(r_{f}\right)=\left\{\begin{array}{l}
\frac{a r_{f}^{3}+b r_{f}^{2}+c r_{f}}{\left(1+r_{f}\right)^{3}}, \quad r_{f} \geq 0, \\
0, \quad r_{f}<0,
\end{array}\right.
$$

em que os parâmetros $a, b$ e $c$ devem ser determinados. Para tanto, impõem-se as condições: (i) $\psi(1)=1$ (para garantir, no mínimo, segunda ordem de exatidão), (ii) $a=\beta$ (variável livre - torna o limitador de fluxo flexível) e (iii) a propriedade de simetria em (B.1). Com a aplicação dessas três condições em (B.2), o limitador resultante é

$$
\psi\left(r_{f}\right)=\left\{\begin{array}{l}
\frac{\beta r_{f}^{3}+(8-2 \beta) r_{f}^{2}+\beta r_{f}}{\left(1+r_{f}\right)^{3}}, \quad r_{f} \geq 0, \\
0, \quad r_{f}<0,
\end{array}\right.
$$

em que $\beta \in[0,2]$. Esta faixa de valores de $\beta$ é estabelecida através de duas restrições que $\psi\left(r_{f}\right)$ deve respeitar para que o limitador seja TVD (ver Figura B.1), são elas:

$$
\left\{\begin{array}{l}
\psi\left(r_{f}\right) \geq 0, \quad \text { para } r_{f} \geq 0, \\
\psi^{\prime}\left(r_{f}\right) \leq 2, \quad \text { para } r_{f} \text { tendendo a } 0 \text { (princípio de monotonicidade de Sweby [139]). }
\end{array}\right.
$$




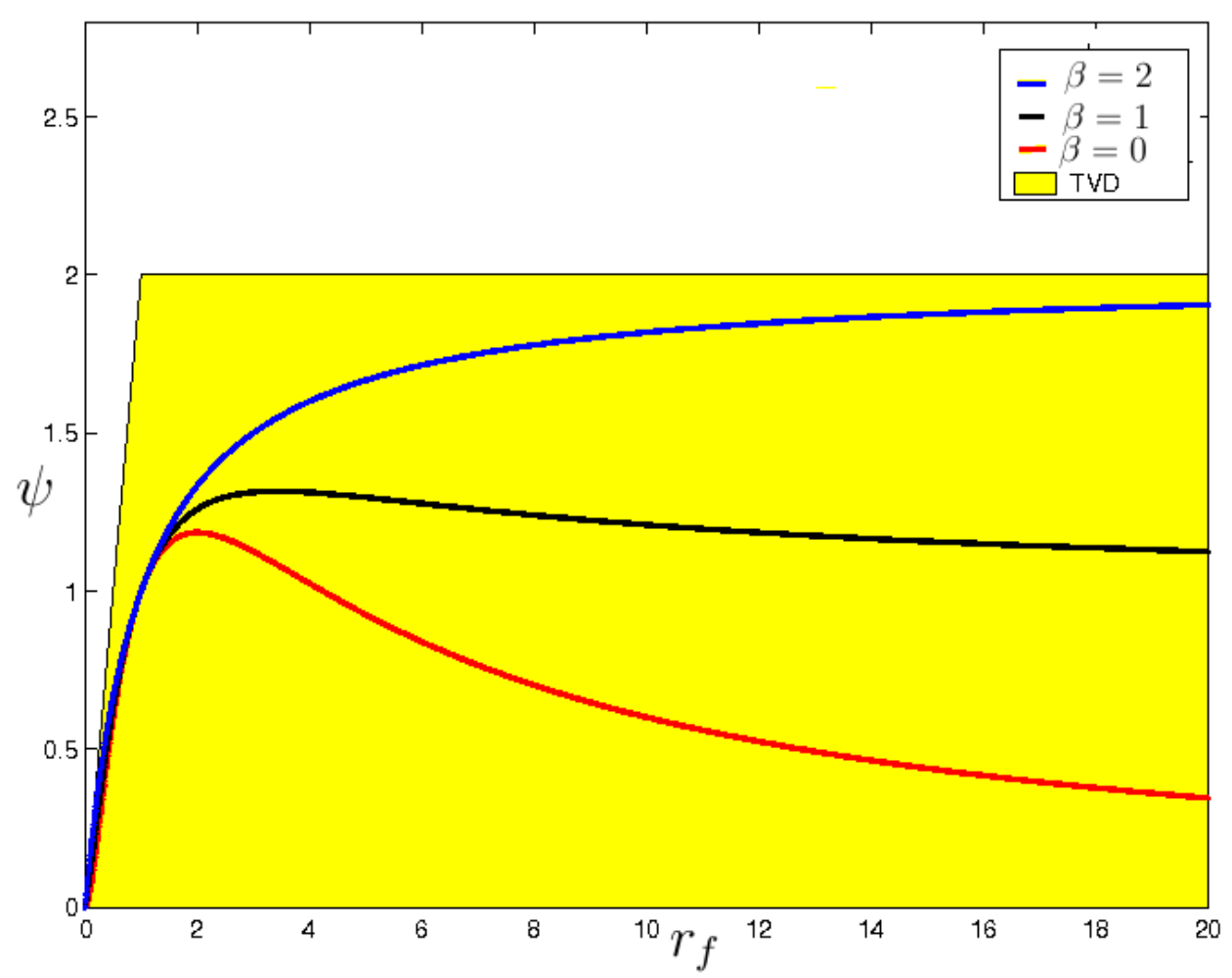

Figura B.1: Curvas características do limitador FSFL na região TVD de Sweby [139].

Da mesma forma que o limitador TOPUS, o limitador FSFL pode ser reescrito por

$$
\psi\left(r_{f}\right)=\max \left\{0, \frac{0.5\left[\left|r_{f}\right|+r_{f}\right)\left(\beta r_{f}^{2}+(8-2 \beta) r_{f}+\beta\right]}{\left(1+\left|r_{f}\right|\right)^{3}}\right\} .
$$

Substituindo (B.3) em (3.11), deriva-se um esquema polinomial "upwind" em NV

$$
\hat{\phi}_{f}=\left\{\begin{array}{l}
(-2 \beta+4) \hat{\phi}_{U}^{4}+(4 \beta-8) \hat{\phi}_{U}^{3}+\left(\frac{-5 \beta+8}{2}\right) \hat{\phi}_{U}^{2}+\left(\frac{\beta+2}{2}\right) \hat{\phi}_{U}, \quad \hat{\phi}_{U} \in[0,1], \\
\hat{\phi}_{U}, \quad \hat{\phi}_{U} \notin[0,1] .
\end{array}\right.
$$




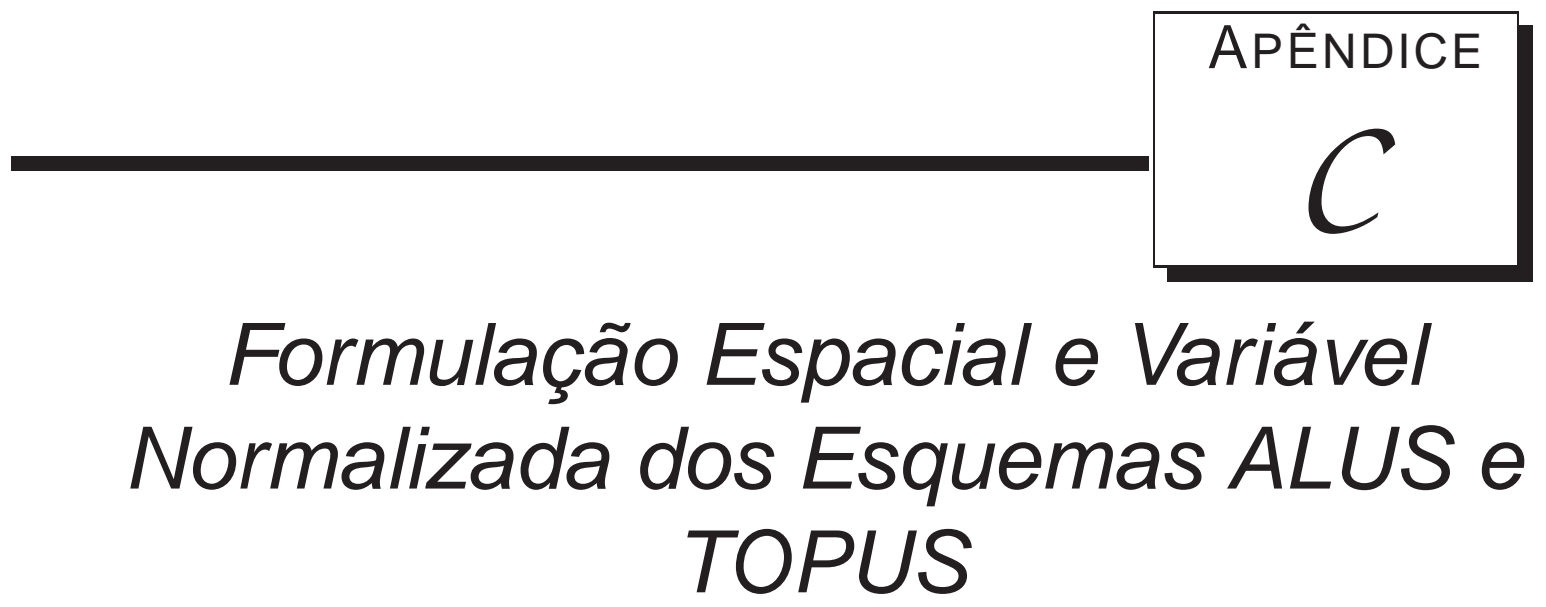

Neste apêndice são apresentados os esquemas ALUS e TOPUS no contexto da metodologia NVSF de Darwish e Moukalled [33] para malhas não uniformes. Essa metodologia amplia a aplicabilidade da abordagem NVF de Leonard [80] para domínios discretizados não uniformemente.

A metodologia NVSF possui a variável normalizada $\hat{\phi}$ da NVF definida em (3.1) e acrescenta

$$
\hat{x}=\frac{x-x_{R}}{x_{D}-x_{R}}
$$

em que $x_{D}$ e $x_{R}$ são, respectivamente, as localizações dos pontos "Downstream" e "Upstream" da malha em relação a origem (veja referência [33] para mais detalhes).

\section{Esquema TOPUS}

O esquema TOPUS no intervalo $\left[x_{R}, x_{D}\right]$ é definido por

$$
\phi=a_{4} x^{4}+a_{3} x^{3}+a_{2} x^{2}+a_{1} x,
$$

sujeito a:

$$
\left\{\begin{array}{l}
\phi=\phi_{R}, \quad \text { para } x=x_{R}, \\
\phi=\phi_{D}, \quad \text { para } x=x_{D}, \\
\phi=\phi_{U}, \quad \text { para } x=x_{U} .
\end{array}\right.
$$

Aplicando as restrições (C.3) em (C.2) e normalizando as variáveis com as definições (3.1) e (C.1), resulta-se o modelo NVSF do TOPUS

$$
\hat{\phi}_{f}=\left\{\begin{array}{l}
\alpha \hat{\phi}_{U}^{4}+a_{3} \hat{\phi}_{U}^{3}+a_{2} \hat{\phi}_{U}^{2}+a_{1} \hat{\phi}_{U}, \quad \alpha \in[-2,2], \quad \hat{\phi}_{U} \in[0,1], \\
\hat{\phi}_{U}, \quad \hat{\phi}_{U} \notin[0,1],
\end{array}\right.
$$


em que

$$
\begin{aligned}
a_{1} & =\frac{-\alpha \hat{x}_{U}^{6}+2 \alpha \hat{x}_{U}^{5}+(1-\alpha) \hat{x}_{U}^{4}-3 \hat{x}_{f} \hat{x}_{U}^{2}+\hat{x}_{f}\left(\hat{x}_{f}+1\right) \hat{x}_{U}}{\hat{x}_{U}^{2}\left(\hat{x}_{U}-1\right)^{2}} \\
a_{2} & =\frac{\alpha \hat{x}_{U}^{6}-3 \alpha \hat{x}_{U}^{4}+2(\alpha-1) \hat{x}_{U}^{3}+3 \hat{x}_{f} \hat{x}_{U}^{2}+\hat{x}_{f}\left(1-\hat{x}_{f}\right) \hat{x}_{U}-\hat{x}_{f}^{2}}{\hat{x}_{U}^{2}\left(\hat{x}_{U}-1\right)^{2}} \\
a_{3} & =\frac{-2 \alpha \hat{x}_{U}^{5}+3 \alpha \hat{x}_{U}^{4}+(1-\alpha) \hat{x}_{U}^{2}+\hat{x}_{f}\left(\hat{x}_{f}-2 \hat{x}_{U}\right)}{\hat{x}_{U}^{2}\left(\hat{x}_{U}-1\right)^{2}}
\end{aligned}
$$

$\hat{x}_{f}$ é o valor normalizado da distância entre a face $f$ do volume de controle e a origem.

\section{Esquema ALUS}

Darwish e Moukalled [33] reformula vários esquemas de alta resolução ( SMART [53], MINMOD [61], MUSCL ("Monotonic Upwind Scheme for Conservation Laws") [151] e outros). Fica claro dessas reformulações (em especial a do MUSCL), que $\hat{\phi}_{f}=2 \hat{\phi}_{U}$ em NVSF é dado por

$$
\hat{\phi}_{f}=\left(\frac{2 \hat{x}_{f}-\hat{x}_{U}}{\hat{x}_{U}}\right) \hat{\phi}_{U} .
$$

Considerando a formulação NVF do ALUS em (3.24), a equação (C.8) e as definições (3.1) e (C.1), a reformulação resultante do esquema ALUS para malhas não uniformes é

$$
\hat{\phi}_{f}= \begin{cases}\left(\frac{2 \hat{x}_{f}-\hat{x}_{U}}{\hat{x}_{U}}\right) \hat{\phi}_{U}, & \hat{\phi}_{U} \in\left[0, \lambda_{a}\right], \\ c_{1} \hat{\phi}_{U}+c_{2}, & \hat{\phi}_{U} \in\left(\lambda_{a}, 1\right], \\ \hat{\phi}_{U}, & \hat{\phi}_{U} \notin[0,1]\end{cases}
$$

em que

$$
\begin{gathered}
\lambda_{a}=\frac{1-|\theta|}{3-|\theta|}, \\
c_{1}=\frac{\hat{x}_{U}+(1-|\theta|)\left(\hat{x}_{U}-\hat{x}_{f}\right)}{\hat{x}_{U}}, \\
c_{2}=\frac{(1-|\theta|)\left(\hat{x}_{f}-\hat{x}_{U}\right)}{\hat{x}_{U}} .
\end{gathered}
$$




\section{APÊNDICE} $\mathcal{D}$

\section{Limitador TOPUS para a simulação de escoamentos aerodinâmicos compressíveis}

Neste apêndice é apresentado o limitador TOPUS TVD (caso $\alpha=2$ )

$$
\psi\left(r_{f}\right)=\frac{\left(r_{f}+\left|r_{f}\right|\right)\left(3 r_{f}+1\right)}{\left(\left|r_{f}\right|+1\right)^{3}}
$$

formulado para simulação de escoamentos aerodinâmicos compressíveis (por exemplo, escoamentos ao redor de aerofólios). Esse limitador, no contexto apresentado por Bigarella [14] (discretização por volumes finitos e $r_{f}=n u m^{ \pm} /$den), é reescrito por $[30,103,109]$

$$
\Psi\left(\text { num }^{ \pm}, \operatorname{den}\right)=\frac{\left(\mid \text { num }^{ \pm} \mid \operatorname{den}+\text { num }^{ \pm}|\operatorname{den}|\right)\left(3 n u m^{ \pm} d e n+\operatorname{den}^{2}+\epsilon_{L I M}\right)}{\operatorname{den}\left(|\operatorname{den}|+\left|n u m^{ \pm}\right|\right)^{3}+\epsilon_{L I M}},
$$

em que $\epsilon_{L I M}$ é um parâmetro de controle do limitador (constante); num ${ }^{ \pm}$e den são determinados, respectivamente, por

$$
\begin{gathered}
\operatorname{num}^{ \pm}=\hat{q}_{i}^{ \pm}-q_{i}, \\
\text { den }=\left(q_{i}\right)_{k}-q_{i} .
\end{gathered}
$$

Nas equações (D.2) e (D.3), têm-se

$$
q(x, y, z)=q_{i}+\nabla q_{i} \cdot \vec{r},
$$

em que $(x, y, z)$ são as coordenadas de um ponto genérico da $i$-ésima célula, $q_{i}$ é o valor discreto da propriedade $q$ na $i$-ésima célula, e $\vec{r}$ denota a distância do centróide da célula ao ponto de coordenadas $(x, y, z)$. Para mais detalhes sobre (D.2), (D.3) e (D.4), veja a tese de Bigarella [14].

Neste trabalho, simulações de escoamentos ao redor de aerofólios com o limitador (D.1) foram realizadas (ver seção 6.2). Para tanto, inicialmente, foi estudada a influência do parâmetro de 
controle $\epsilon_{L I M}$ na convergência do método numérico de Roe de segunda ordem [29, 65, 117]. Foram testados $\epsilon_{L I M}$ com os valores $10^{-1}, 10^{-2}, 10^{-4}, 10^{-6}, 10^{-8}$ e $10^{-10}$. Nesses testes ocorreu convergência do método numérico, com exceção para $\epsilon_{L I M}=10^{-10}$ (ver referência [30]). Vale mencionar que foi escolhido $\epsilon_{L I M}=10^{-8}$ para o limitador TOPUS nas simulações de escoamentos compressíveis descritas neste trabalho. 


\section{Referências Bibliográficas}

[1] R. Ahmed. Numerical schemes applied to the burgers and buckley-leverett equations, 2004. Msc dissertation.

[2] K. Alhuimaizi. Flux-limiting solution techniques for simulation of reaction-diffusion-convection system. Communications in Nonlinear Sciences and Numerical Simulation, 12:953-965, 2007.

[3] M.A. Alves, P.J. Oliveira, and E.T. Pinho. A convergent and universally bounded interpolation for the treatment of advection. International Journal for Numerical Methods in Fluids, 41:47-75, 2003.

[4] A.A. Amsden and F.H. Harlow. A simplified mac technique for incompressible fluid flow calculations. Journal of Computational Physics, 6:322-325, 1970.

[5] D.A. Anderson, J.C. Tannehill, and R.H. Pletcher. Computational fluid mechanics and heat transfer. Hemisphere Publishing Corporation, New York, 1984.

[6] J. D. Anderson. Modern Compressible Flow: With Historical Perspective. McGraw-Hill, 1990.

[7] R. Ansorge. Mathematical Models of Fluiddynamics. Wiley-VCH Verlag GmbH \& Co. KGaA, 2003.

[8] B.F. Armaly, F. Durst, J.C.F. Periera, and B. Schonung. Experimental and theoretical investigation of backward facing step flow. Journal of Fluid Mechanics, 127:473-496, 1983.

[9] S. W. Armfield. Finite difference solutions of the navier-stokes equations on staggered and non-staggered grids. Computers \& Fluids, 20:1-17, 1991.

[10] M. Arora and P.L. Roe. A well-behaved tvd limiter for high-resolution calculations of unsteady flow. Journal of Computational Physics, 132:3-11, March 1997.

[11] U.M. Ascher and L.R. Petzold. Computer methods for ordinary differential equations and differential-algebraic equations. SIAM, 1998.

[12] C.T. Avedisian and Z. Zhao. The circular hydraulic jump in low gravity. In Proceedings of the Royal Society of London, Series A, pages 2127-2151, 2000. 
[13] E.R. Berton and W.G. Platzman. A table of solutions of the one-dimensional burgers equation. Quarterly of Applied Mathematics, pages 195-212, 1972.

[14] E.D.V. Bigarella. Advanced Turbulence Modeling for Complex Aerospace Applications. $\mathrm{PhD}$ thesis, Instituto Tecnológico de Aeronáutica, São José dos Campos, SP, 2007.

[15] R.B. Bird, R.C. Armstrong, and O. Hassager. Dynamics of polymeric liquids. John Willey \& Sons, 2nd edition, May 1987.

[16] J. Boussinesq. Memoires presentes par divers savants sciences mathematique at physiques theorie de l'ecoulement tourbillant. Academie des Sciences, Paris, 1877.

[17] A.C. Brandi. Estratégias "upwind" e modelagem $\kappa-\varepsilon$ para simulação numérica de escoamentos com superfícies livres em altos números de reynolds, 2005. Dissertação de mestrado - ICMC/USP.

[18] J. Burguete, P. García-Navarro, and J. Murillo. Preserving bounded and conservative solutions of transport in one-dimensional shallow-water flow with upwind numerical schemes: Application to fertigation and solute transport in rivers. International Journal for Numerical Methods in Fluids, 456:1731-1764, 2008.

[19] J.W.M. Bush, J.M. Aristoff, and A.E. Hosoi. An experimental investigation of the stability of the circular hydraulic jump. Journal of Fluid Mechanics, 558:33-52, 2006.

[20] A. Castelo, M. F. Tomé, C. N. L. César, S. McKee, and J. A. Cuminato. Freeflow: An integrated simulation system for three-dimensional free surface flows. Journal of Computing and Visualization in Science, 2:199-210, 2000.

[21] V. Casulli and P. Zanolli. A practical method for numerical evaluation of solutions of partial differential equations of the heat-conduction type. Ocean Modelling, 10:137-151, 2005.

[22] T. L. Chambers and D. C. Wilcox. Critical examination of two-equation turbulence closure models for boundary layers. AIAA Journal, 15:821-828, 1977.

[23] A. J. Chorin. Numerical solution of the navier-stokes equations. Mathematics of Computation, 2:745-762, october 1968.

[24] C. Corre and A. Lerat. High-order residual-based compact schemes for advection-diffusion problems. Computers \& Fluids, 37:505-519, 2008.

[25] R. Courant, E. Isaacson, and M. Rees. On the solution of nonlinear hyperbolic differential equations by finite differences. Community Pure Applied Mathematics, 5:243 -255, 1952.

[26] J. Crank and P. Nicolson. A practical method for numerical evaluation of solutions of partial differential equations of the heat-conduction type. In Mathematical Proceedings of the Cambridge Philosophical Society, pages 50-67, 1947.

[27] J.O. Cruickshank. Low-reynolds-number instabilities in stagnating jet flows. Journal of Fluid Mechanics, 193:111-127, 1987. 
[28] J.O. Cruickshank and B.R. Munson. Viscous fluid buckling of plane and axisymmetric jets. Journal of Fluid Mechanics, 113:221-239, 1981.

[29] R.G. Cuenca. Estudo de otimização de desempenho de aerofólios super-críticos utilizando algoritmo genéticos, 2009. Dissertação de mestrado - ICMC/USP.

[30] R.G. Cuenca, R.A.B. Queiroz, L.F. Souza, and V.G. Ferreira. Evaluation of a new tvd limiter for transonic and supersonic flows. In Proceedings of the 12th Brazilian Congress of thermal sciences and engineering - ENCIT, 2008.

[31] R.A. Dalrymple, O. Knio, D.T. Cox, M. Gesteira, and S. Zou. Using a lagrangian particle method for deck overtopping. In Proceedings of the Waves 2001, ASCE, pages 1082-1091, 2002.

[32] B.J. Daly and F.H. Harlow. Transport equations in turbulence. Physics of Fluids, 13:2634-2649, 1970.

[33] M. S. Darwish and F. Moukalled. Normalized variable and space formulation methodology for high-resolution schemes. Numerical Heat Transfer, 26:79 - 96, 1994.

[34] F.M. Denaro. On the applications of the helmoltz-hodge decomposition in projection methods for incompressible flows with general boundary conditions. International Journal for Numerical Methods in Fluids, 43:43-69, 2003.

[35] P.A. Durbin. On the $\kappa-\varepsilon$ stagnation point anomaly. Journal Heat and Fluid Flow, 17:89 $-90,1996$.

[36] J. Eaton and J. P. Johnston. Turbulent flow reattachment: an experimental study of the flow and structure behind a backward-facing step. Technical Report TR MD-39, Stanford University, 1980.

[37] C. Ellegaard, A.E. Hansen, A. Haaning, and T. Bohr. Experimental results on flow separation and transitions in the circular hydraulic jump. Physica Scripta, T67:105-110, 1996.

[38] C. Ellegaard, A.E. Hansen, A. Haaning, K. Hansen, A. Marcussen, and T. Bohr. Poligonal hydraulic jumps. Nonlinearity, 12:1-7, 1999.

[39] C. Ellegaard, A.E. Hansen, A. Haaning, K. Hansen, A. Marcussen, T. Bohr, J. Lundbek, and S. Watanabe. Creating corners in kitchen sinks. Nature, 392:787-788, 1998.

[40] I. Fatkullin and J.S. Hesthaven. Adaptive high-order finite-difference method for nonlinear wave problems. Journal of Scientific Computing, 16:47-67, March 2001.

[41] R. Fazio and R.J. LeVeque. Moving-mesh methods for one-dimensional hyperbolic problems using clawpack. Computers \& Mathematics with Applications, 45:273-298, 2003.

[42] V. G. Ferreira. Análise e implementação de esquemas de convecção e modelos de turbulência para simulação de escoamentos incompressíveis envolvendo superfícies livres. $\mathrm{PhD}$ thesis, Instituto de Ciências Matemáticas e de Computação (ICMC-USP), 2001. 
[43] V. G. Ferreira. Contribuição para o desenvolvimento de esquemas "upwind" e suas aplicações em dinâmica dos fluidos, 2008. Tese de livre docência, Instituto de Ciências Matemáticas e de Computação (ICMC-USP).

[44] V. G. Ferreira, N. Mangiavacchi, M. F. Tomé, A. Castelo, J. A. Cuminato, and S. McKee. Numerical simulation of turbulent free surface flow using two-equation $\kappa-\varepsilon$ viscosity model . International Journal for Numerical Methods in Fluids, 44:347-375, 2004.

[45] V. G. Ferreira, M. F. Tomé, N. Mangiavacchi, A. Castelo, J. A. Cuminato, A. O. Fortuna, and S. Mckee. High-order upwinding and the hydraulic jump. International Journal for Numerical Methods in Fluids, 39:549-583, 2002.

[46] V.G. Ferreira, A.C. Brandi, F.A. Kurokawa, P. Seleghim Jr., A. Castelo, and J.A. Cuminato. Incompressible turbulent flow simulation using $\kappa-\varepsilon$ model and upwind schemes. Mathematical Problems in Engineering, 2007.

[47] V.G. Ferreira, F.A. Kurokawa, C.M. Oishi, M.K. Kaibara, A. Castelo, and J.A. Cuminato. Evaluation of a bounded high order upwind scheme for $3 \mathrm{~d}$ incompressible free surface flow computations. Mathematics and Computers in Simulation, In Press.

[48] V.G. Ferreira, F.A. Kurokawa, R.A.B. Queiroz, M.K. Kaibara, C.M. Oishi, J.A. Cuminato, A. Castelo, M.F. Tomé, and S. Mckee. Assessment of a high-order finite difference upwind scheme for the simulation of convection-diffusion problems. International Journal for $\mathrm{Nu}-$ merical Methods in Fluids, In Press.

[49] V.G. Ferreira, C.M. Oishi, M.K. Kaibara F.A. Kurokawa, J.A. Cuminato, A. Castelo, M.F. Tomé, and S. Mckee. A combination of implicit and adaptative upwind tools for numerical solution of incompressible free surface flows. Communications in Numerical Methods in Engineering, 23:419-445, 2007.

[50] V.G. Ferreira, R.A.B. Queiroz, F.A. Kurokawa, R.G. Cuenca, C.M. Oishi, G. A. B. Lima, and S. Mckee. An upwind differencing scheme for conservation laws and related fluid dynamics problems, 2009. Submitted to Journal of Computational Physics, Elsevier.

[51] J.A. Ferziger and M. Perić. Computational methods for fluid dynamics. Springer, 1984.

[52] E. Fontaine, M.E. Landrini, and M. Tulin. Breaking: Splashing and ploughing phases. In Proceedings of International Workshop on Waterwaves and Floating Bodies, pages 34-38, 2000.

[53] P.H. Gaskell and A.K.C. Lau. Curvature-compensated convective transport: Smart, a new boundedness preserving transport algorithm. International Journal for Numerical Methods in Fluids, 8:617-641, 1988.

[54] T. B. Gatski and C. G. Speziale. On explicit algebraic stress models for complex turbulent flows flows. Journal of Fluid Mechanics, 254:59-78, 1993.

[55] S. S. Girimaji. Fully-explicit and self-consistent algebraic reynolds stress model. ICASE, pages 95-82, 1995. 
[56] J. Glimm. Solution in the large for nonlinear hyperbolic systems of equations. Communications on Pure and Applied Mathematics, 18:697-715, 1965.

[57] E.S. Gross, J.R. Koseff, and S.G. Monismith. Evaluation of advective scheme for estuarine salinity simulation. Journal of Hydraulic Engineering, 125:32-46, 1999.

[58] L. Grossi. Desenvolvimento de métodos numéricos para a simulação de escoamentos não-newtonianos e viscoelástico com superfície livre. PhD thesis, Instituto de Ciências Matemáticas e de Computação (ICMC-USP), 2003.

[59] M. Gómez-Gesteira and R.A. Dalrymple. Using a three-dimensional smoothed particle hydrodynamics method for wave impact on a tall structure. Journal of Waterway, Port. Coastal, and Ocean Engineering, 130:63-69, 2004.

[60] F. H. Harlow and J. E. Welch. Numerical calculations of time-dependent viscous incompressible flow of fluid with free surface. Physics of Fluids, 8:2182-2189, 1965.

[61] A. Harten. High resolution schemes for hyperbolic conservation laws. Journal of Computational Physics, 49:357-393, 1983.

[62] A. Harten. Eno schemes with subcell resolution. Journal of Computational Physics, 83:148-184, 1989.

[63] A. Harten, B. Engquist, S. Osher, and S. Chakravarthy. Uniformly high order essentially nonoscillatory schemes. Journal of Computational Physics, 71:303-321, 1987.

[64] M. R. Hestenes and E. Stiefel. Methods of conjugate gradients for solving linear systems. Journal of Research of the National Bureau of Standards, 49:409-436, 1952.

[65] C. Hirsch. Numerical computation of internal and external flows. Wiley, Chichester, vols 1 and 2 edition, 1990.

[66] A. Jameson, W. Schimidt, and E. Turkel. Numerical solutions of the euler equations by finite volume methods using runge-kutta time stepping schemes. AIAA Journal, 81:1259, 1981.

[67] M. K. Kaibara, V. G. Ferreira, and H. A. Navarro. Upwinding finite-difference schemes for convection dominated problems, 2004. Part I, Theoretical Results, Notas do ICMC-USP, n. 80 .

[68] V.I. Karpman. Non-linear Waves in Dispersive Media. Pergamon, New York, 1975.

[69] J.M. Kim, K.H. Ahn, and S.J. Lee. Numerical simulation of moving free surface problems in polymer processing using volume-of-fluid method. Polymer Engineering and Science, 41:858-866, 2001.

[70] S. Koshizuka and Y. Oka. Moving-particle semi-implicit method for fragmentation of incompressible fluids. Nuclear Science and Engineering, 123:421-434, 1996.

[71] R. Kumar and M.K. Kadalbajoo. A class of high resolution shock capturing schemes for hyperbolic conservation laws. Applied Mathematics and Computations, 195:110-126, 2008. 
[72] R. Kupferman and E. Tadmor. A fast, high-resolution, second-order central scheme for incompressible flows. In National Academy of Sciences of the United States of America, pages 4848-4852, USA, 1997.

[73] M. Kurihara. A consideration about hydraulic jump. Technical Report 3:11-25, Kyusyu Imperial University, 1946.

[74] F. A. Kurokawa, V. G. Ferreira, C. M. Oishi, and M. K. Kaibara. A comparison of high order upwind schemes for solving strong convection problems. In Proceedings of the XXVIII CILAMCE - Congresso Ibero Latino-Americano sobre Métodos Computacionais em Engenharia, 2007.

[75] F.A. Kurokawa, R.A.B. Queiroz, and V.G. Ferreira. The performance of a high order upwind scheme for the numerical simulation of fluid flow problems. In Proceedings of the 7th Brazilian Conference On Dynamics, Control and Applications - DINCON, 2008.

[76] B. E. Launder and D. B. Spalding. The numerical computation of turbulent flows. Computer Methods in Applied Mechanics and Engineering, 3:269-289, 1974.

[77] P.D. Lax and B. Wendroff. Systems of conservation laws. Communications on Pure and Applied Mathematics, 13:217-237, 1960.

[78] H. Lei, L-Z. Wang, and Z-N.Wu. Applications of upwind and downwind schemes for calculating electrical conditions in a wire-plate electrostatic precipitator. Journal of Computational Physics, 193:697-707, 2004.

[79] B. P. Leonard. A stable and accurate convective modelling procedure based on quadratic interpolation. Computer Methods in Applied Mechanics and Engineering, 19:59-98, 1979.

[80] B. P. Leonard. Simple high-accuracy resolution program for convective modeling of discontinuities. International Journal for Numerical Methods in Fluids, 8:1291-1318, 1988.

[81] B.P. Leonard. The quick algorithm: a uniformly third-order finite difference method for highly convective flows. Computer Methods in Applied Mechanics and Engineering, 71:321-303, 1979.

[82] R.J. LeVeque. Finite Volume Methods for Hyperbolic Problems. Cambridge Texts in Applied Mathematics, 2002.

[83] R.J. LeVeque. Finite difference methods for ordinary and partial differential equations, steady-state and time-dependent problems. SIAM Society for Industrial and Applied Mathematics, 2007.

[84] C.-L. Lin, H. Lee, T. Lee, and L. J. Weber. A level set characteristic galerkin finite element method for free surface flows. International Journal for Numerical Methods in Fluids, 49:521-547, 2005.

[85] P.-J. Lu and K.-C. Wu. Assessment of total variation diminishing schemes in compressible mixing flow computations. AIAA Journal, 30:939-946, 1992. 
[86] R. Löhner, C. Yang, and E. Onate. Simulation of flows with violent free surface motion and moving objects using unstructured grids. International Journal for Numerical Methods in Fluids, 53:1315-1338, 2007.

[87] J. Martin and W. Moyce. An experimental study of the collapse of liquid columns on a rigid horizontal plate. Philosophical Transactions of Royal Society of London, 244:312-324, 1952.

[88] F.P. Martins. Desenvolvimento de um método numérico implícito para a simulação de escoamentos viscoelásticos com superfícies livres, 2009. Dissertação de mestrado - ICMC/USP.

[89] MFIX. Mfix - multiphase flow with interphase exchanges, 2009. Disponível em https://mfix.netl.doe.gov/index.php, Acesso em: Janeiro/2009.

[90] S. Middleman. Modeling Axisymmetric Flows: Dynamics of Films, Jets, and Drops. Academic Press, 1995.

[91] J.P Morris, P.J. Fox, and Y. Zhu. Modeling low reynolds number incompressible flows using sph. Journal of Computational Physics, pages 214 -226, 1997.

[92] K.W. Morton and D.F. Mayers. Numerical Solution of Partial Differential Equations. Cambridge University Press, second edition edition, April 2005.

[93] D. Mouaze, F. Murzyn, and J.R. Chaplin. Free surface length scale estimation in hydraulics jumps. Journal of Fluids Engineering, 127:1191-1193, 2005.

[94] L.R. Mudryk and N.W. Murray. Rapid: A fast resolution flux-conservative algorithm designed for planet-disk interactions. New Astronomy, In Press.

[95] H. L. Norris and W. C. Reynolds. Turbulent channel flow with a moving wavy boundary. Technical Report TR TF-7, Department of Mechanics Engineering, 1975.

[96] C.M. Oldenburg and K. Pruess. Simulation of propagating fronts in geothermal reservoirs with the implicit leonard total variation diminishing scheme. Geothermics, 29:1-25, 2000.

[97] OpenFOAM. Openfoam - the open source cfd toolbox, 2009. Disponível em http://www.opencfd.co.uk/, Acesso em: Janeiro/2009.

[98] A. Ouahsine and H. Smaoui. Flux-limiter schemes for oceanic tracers: application to the english channel tidal model. Computer Methods in Applied Mechanics and Engineering, 179:307-325, 1999.

[99] A. Pascau and C. Perez. A well-behaved scheme to model strong convection in a general transport equation. In Proceedings of the Eighth International Conference on Numerical Methods in Laminar and Turbulent Flow, page 608, Swansea, July 1993. Pineridge Press.

[100] V. C. Patel. Perspective: Flow at high reynolds number and over rough surfaces-achilles heel of cfd. Transaction of the ASME Journal on Fluids Engineering, 120:434-444, 1998.

[101] R. Peyret and T. D. Taylor. Computational methods for fluid flow. Springer-Verlag, New York, USA, 1983. 
[102] T.M. Pimenta, F.A. Kurokawa, R.A.B. Queiroz, and V.G. Ferreira. Avaliação computacional de esquemas de alta resolução em problemas de dinâmica dos fluidos. Artigo submetido no periódico Tema - Tendências em Matemática Aplicada e Computacional.

[103] R.A.B. Queiroz, R.G. Cuenca, V.G. Ferreira, and L.F. Souza. A new high resolution tvd scheme for unsteady flows with shock waves. In Proceedings of the 7th Brazilian Conference On Dynamics, Control and Applications - DINCON, 2008.

[104] R.A.B. Queiroz, V. G. Ferreira, and R.G. Cuenca. A high resolution cbc/tvd upwind scheme for unsteady flows with shock waves, 2008. Submitted to the Turkish Journal of Engineering and Environmental Sciences.

[105] R.A.B. Queiroz and V.G. Ferreira. Esquemas polinomiais upwind e suas aplicações em escoamentos incompressíveis $3 \mathrm{~d}$ transientes. In Anais do XIV Congresso Nacional de Estudantes de Engenharia Mecânica - XIV CREEM, 2007.

[106] R.A.B. Queiroz and V.G. Ferreira. Esquemas polinomiais upwind para problemas de dinâmica dos fluidos computacional. In Anais do XIII Encontro de Iniciação Científica e Pós-Graduação do ITA - XIII ENCITA, 2007.

[107] R.A.B. Queiroz and V.G. Ferreira. Stress analysis of a plate with a hole using an open source tool. In Proceedings of the 6th Brazilian Conference on Dynamics, Control and Their Applications, 2007.

[108] R.A.B. Queiroz and V.G. Ferreira. Numerical simulation of turbulent free surface flows using a combination of a new high order upwind scheme and a realizable reynolds stress algebraic model, 2008. Talk to 3rd LNCC Meeting on Computational Modelling, Petrópolis-RJ.

[109] R.A.B. Queiroz, V.G. Ferreira, and R.G. Cuenca. A new high resolution tvd scheme for unsteady flows with shock waves. Tendências em Matemática Aplicada e Computacional TEMA, 9:311-320, 2008.

[110] R.A.B. Queiroz, V.G. Ferreira, and F.A. Kurokawa. Desenvolvimento e aplicação de esquemas upwind de terceira ordem para transporte convectivo. In Anais do XXX Congresso Nacional de Matemática Aplicada e Computacional - XXX CNMAC, 2007.

[111] R.A.B. Queiroz, V.G. Ferreira, and F.P. Martins. A new upwind scheme for numerical solution of fluid dynamics problems. In Anais do I Encontro Regional de Matemática Aplicada e Computacional - ERMAC, 2008.

[112] R.A.B. Queiroz, F.A. Kurokawa, and V.G. Ferreira. Direct computation of incompressible turbulent free surface flow using a new high order upwind scheme. In Anais da Escola de Primavera e Transição e Turbulência - EPTT, 2008.

[113] R.A.B. Queiroz, F.A. Kurokawa, and V.G. Ferreira. New high order upwind techniques for advective term discretizations. In Proceedings of the 12th Brazilian Congress of thermal sciences and engineering - ENCIT, 2008. 
[114] R.A.B. Queiroz, F.A. Kurokawa, and V.G. Ferreira. A polynomial upwind scheme for convection discretization. In Anais do XXXI Congresso Nacional de Matemática Aplicada e Computacional-XXXI CNMAC, 2008.

[115] R.A.B. Queiroz, F.A. Kurokawa, V.G. Ferreira, and F.P. Martins. Development and implementation of polynomial scheme for the numerical solution of $1 \mathrm{~d}$ conservation laws. In Anais do VIII Simpósio Mecânica Computacional - SIMMEC, 2008.

[116] D. Rajagopalan, R. Amstrong, and R. Brown. Finite element methods for calculation of steady viscoelastic flow using constitutive equations with newtonian viscosity. Journal of Non-Newtonian Fluid Mechanics, 36:159-192, 1990.

[117] P. L. Roe. Approximate riemann solvers, parameter vectors and difference schemes. Journal of Computational Physics, 43:357-372, 1981.

[118] P.L. Roe. Some contributions to the modelling of discontinuous flows. In Lectures in Applied Mathematics, Large-scale Computations in Fluid Mechanics, 1985.

[119] P.L. Roe. Characteristic-based schemes for the euler equations. In Annual review of fluid mechanics, pages 337-365, 1986.

[120] S. Le Roy, G. Teyssendre, and G. Laurent. Numerical methods in the simulation of charge transport in solid dielectrics. IEEE Transaction on Dielectrics and Electrical Installation, 13:239-246, 2006.

[121] P. Sagaut. Large Eddy Simulation for Incompressible Flows-An Introduction. Springer, 2001.

[122] S. Sankaranarayanan and H. Suresh Rao. Finite element analysis of free surface flow through gates. International Journal for Numerical Methods in Fluid, 22:375-392, 1996.

[123] T. H. Shih, J. Zhu, and J. L. Lumley. A realizable reynolds stress algebraic equation model. Technical Report TM 105993, NASA, 1993.

[124] C.-W. Shu and S. Osher. Efficient implementation of essentially non-oscillatory shock capturing schemes. Journal of Computational Physics, 83, 1989.

[125] W. Shyy, S. Thakur, and J. Wright. Second-order upwind and central difference schemes for recirculating flow computation. AIAA Journal, 30:923-932, 1992.

[126] G.F. Silva. Simulação numérica de escoamentos viscoelásticos com superfície livre usando o ambiente freeflow-2d, 2003. Dissertação de mestrado - ICMC/USP.

[127] J.M Silva. Modelagem numérica do escoamento num tubo permeável aplicada ao processo de filtração tangencial. PhD thesis, Escola de Engenharia de São Carlos - Universidade de São Paulo, São Carlos, 2008.

[128] J.W. Slater. $\quad$ Rae 2822 transonic airfoil, 2008. http://www.grc.nasa.gov/WWW/wind/valid/raetaf/raetaf04/raetaf04.html, Acesso: Março/2008. 
[129] G.D. Smith. Numerical Solution of Partial Differential Equations: Finite Difference Methods. Clarendon Press, third edition edition edition, 1999.

[130] G. Sod. A survey of several finite difference methods for systems of nonlinear hyperbolic conservation laws. Journal of Computational Physics, 27, 1978.

[131] D. L. Sondak and R. H. Pletcher. Application of wall functions to generalized nonorthogonal curvilinear coordinate systems. AIAA Journal, 33:33-41, 1995.

[132] D.L. Sondak, R.H. Pletcher, and W.R. Vandalsem. Wall functions for the kappa-epsilon turbulence model in generalized nonorthogonal curvilinear coordinates. Technical Report N-92-25962, Iowa State Univ. of Science and Technology, Ames, IA,United States, 1992.

[133] B. Song, G.R. Liu, K.Y. Lam, and R. S. Amano. On a higher-order bounded discretization scheme. International Journal for Numerical Methods in Fluids, 32:881-897, 2000.

[134] P.R. Spalart. Strategies for turbulence modeling and simulations. International Journal Heat Fluid Flow, 21:252-263, 2000.

[135] C. G. Speziale. Turbulence modeling for time-dependent rans and vles: a review. AIAA Journal, 36:173-184, 1998.

[136] C. G. Speziale and S. Thangam. Analysis of an rng based turbulence model for separated flows. Technical Report ICASE Report - N. 92-93, NASA CR-189600, 1992.

[137] P.K. Stansby, A. Chegini, and T.C.D. Barnes. The initial stages of dam-break flow. Journal of Fluid Mechanics, 374:407-424, 1998.

[138] E.R. Stuart and K. Dochan. An upwind differencing scheme for incompressible navier-stokes equations. Applied Numerical Mathematics, 8:43-64, 1991.

[139] P.K. Sweby. High resolution scheme using flux limiters for hyperbolic conservation laws. SIAM Journal on Numerical Analysis, 21:995-1011, 1984.

[140] N. Takemitsu. Finite difference method to solve incompressible fluid flow. Journal of Computational Physics, 61:499-518, 1985.

[141] I. Tani. Flow separation in thin liquid layers. Journal of the Physics Society of Japan, 4:212-215, 1948.

[142] G.I. Taylor. Low-reynolds number flows, 1974. National Committee for Fluid Mechanics Films. Illustrated experiments in fluid mechanics.

[143] J.J. Thibert, M. Grandjacques, and L.H. Ohman. Experimental database for computer program assessment. TR AR-138, AGARD.

[144] V. A. Titarev and E. F. Toro. Weno schemes based on upwind and centred tvd fluxes. Computers and Fluids, 34:705-720, 2005. 
[145] M.F. Tomé, A. Castelo, J. A. Cuminato, and S. McKee. Gensmac3d: A numerical method for solving unsteady three-dimensional free surface flows. International Journal for Numerical Methods in Fluids, 37:747-796, 2000.

[146] M.F. Tomé and S. McKee. Gensmac: A computational marker-and-cell method for free surface flows in general domains. Journal of Computational Physics, 116:171-186, 1994.

[147] E. F. Toro. Riemann Solvers and Numerical methods for fluid Dynamics. Springer, a pratical introdution edition, 1991.

[148] G. Toth and D. Odstrcil. Comparison of some flux corrected transport and total variation diminishing numerical schemes for hidrodynamic and magnetohydrodynamic problems. Journal of Computational Physics, 128:82-100, 1996.

[149] H. Trac and H.-L. Pen. A primer on eulerian computational fluid dynamics for astrophysics. Publication of the Astronomical Society of the Pacific, 115:303-321, 2003.

[150] G.D. van Albada, B. van Leer, and W. W. Roberts. A comparative study of computational methods in cosmic gas dynamics. Astronomy and Astrophysics, 108:76-84, 1982.

[151] B. van Leer. Towards the ultimate conservative difference scheme. v. a second-order sequel to godunov's method. Journal of Computational Physics, 23:101-136, 1977.

[152] A. Varonos and G. Bergeles. Development and assessment of a variable-order non-oscillatory scheme for convection term discretization. International Journal for $\mathrm{Nu}$ merical Methods in Fluids, 26:1-16, 1998.

[153] R. F. Warming and R. M. Beam. Upwind second-order difference schemes and application in aerodynamics flows. AIAA Journal, 14:1241-1249, 1976.

[154] N. P. Waterson and H. Deconinck. Design principles for bounded higher-order convection schemes - a unified approach. Journal of Computational Physics, 224:182-207, May 2007.

[155] E.J. Watson. The radial spread of a liquid jet over a horizontal plane. Journal of Fluid Mechanics, 20:481-499, 1964.

[156] G. W. Wei and Y. Gu. Conjugated filter approach for solving burger's equation. Journal of Computational and Applied Mathematics, 149:439-456, 2002.

[157] F.M. White. Fluid Mechanics. McGraw-Hill, 1979.

[158] G.B. Whitham. Linear and Nonlinear Waves. John Wiley \& Sons, New York, 1974.

[159] D. C. Wilcox. Turbulence Modeling for CFD. DCW Industries, third edition, 2006.

[160] W.R. Wolf. Simulações de escoamentos aerodinâmicos compressíveis utilizando esquemas não oscilatórios em malhas não estruturadas. PhD thesis, Instituto Tecnológico de Aeronáutica, São José dos Campos, SP, 2006.

[161] Y.H. Zahran. Third order tvd scheme for hyperbolic conservation laws. Bulletin of Belgian Mathematical Society, 14:259-275, 2007. 
[162] Y.H. Zahran. Rcm-tvd hybrid scheme for hyperbolic conservation laws. International Journal for numerical Methods in Fluids, 57:745-750, 2008.

[163] X. Zhao, P.G. Richards, S.J. Zhang, and J. Liu. High-resolution schemes for bubbling flow computations. Applied Mathematical Modelling, 29:1232-1251, 2005.

[164] G. Zhou. Numerical simulations of physical discontinuities in single and multi-fluid flows for arbitrary Mach numbers. PhD thesis, Chalmers University of Technology, Sweden, 1995.

[165] G. Zhou, L. Davidson, and E. Olsson. Transonic inviscid/turbulent airfoil flow simulations using a pressure-based method with high order schemes. Lecture Notes in Physics, 453:372-377, 1995.

[166] J. Zhu. On the higher-order bounded discretization schemes for finite volume computations of incompressible flows. Computer Methods in Applied Mechanics and Engineering, 98:345-360, 1992.

[167] M. Zijlema. Computational modeling of turbulent flow in general domains. PhD thesis, Delft University of Technology, The Netherlands, 1996.

[168] M. Zijlema. On the construction of a third-order accurate monotone convection scheme with application to turbulent flows in general domains. International Journal for Numerical Methods in Fluids, 22:619-641, 1996. 